\title{
Development of Transferable Coarse-Grained Models of Amino Acids
}

\section{Olivia Kristine Conway}

Thesis submitted to the faculty of the Virginia Polytechnic Institute and State University in partial fulfillment of the requirements for the degree of

\section{Master of Science}

In

Chemical Engineering

Sanket A. Deshmukh

Luke E. K. Achenie

John B. Matson

August 27, 2019

Blacksburg, VA

Keywords: molecular dynamics, coarse grained, amino acids, peptide amphiphile, self-assembly 


\section{Development of Transferable Coarse-Grained Models of Amino Acids \\ Olivia Kristine Conway \\ ABSTRACT}

There are twenty standard amino acids that are the structural units of biomolecules and biomaterials such as proteins and peptide amphiphiles (PAs). The focus of this study was to develop accurate transferable coarse-grained (CG) models of those amino acids. In CG models, several atoms are represented together as a single pseudo-atom or "bead," which can allow the modeling of processes like self-assembly of biomolecules and biomaterials through reduction of degrees of freedom and corresponding increased computational speed. A 2:1 to 4:1 mapping scheme, in which a CG bead is comprised of two to four heavy atoms, respectively, and associated hydrogens, has been employed to represent functional groups in the amino acids. The amino acid backbone atoms are modeled as two beads while the side chains are modeled with one to three beads, and each terminus is modeled as one bead. The bonded parameters for the CG models were obtained from bond, angle, and dihedral distributions from all-atom molecular dynamics (MD) simulations of dipeptides. Non-bonded parameters were optimized using the particle swarm optimization (PSO) method to reproduce experimental properties (heat of vaporization, surface tension, and density) of analogues of the side chains, termini, and backbone groups of the amino acids. These CG models were used to study the self-assembly pathways and mechanisms of the PA c16- $\mathrm{AHL}_{3} \mathrm{~K}_{3}-\mathrm{CO}_{2} \mathrm{H}$ in the presence of explicit CG water. 


\section{Development of Transferable Coarse-Grained Models of Amino Acids \\ Olivia Kristine Conway \\ GENERAL AUDIENCE ABSTRACT}

In this study, models of the amino acids were developed for computer simulations. In these models, the amino acids are represented as a collection of two or more "beads" bonded together rather than as a collection of atoms bonded together. The beads were created in such a way that their characteristics reflect those of the molecules and atom groups that they represent. This was accomplished in part by selecting parameters for each bead that approximately reproduce experimental properties (density, heat of vaporization, and surface tension) and structures (bonds and angles) of the molecules and atom groups of which they are representative. Amino acids can link together to form short segments, known as peptides, or longer chains that form proteins. The bead models that were developed in this study can be linked together in the same way. They can also be linked with other beads that represent other atom groups-carbon groups of a carbon chain, for example. Certain types of molecules known as peptide amphiphiles (PAs) are often composed of amino acids and a carbon chain. The amino acid bead models were created especially to study these molecules, so once the models were developed, they were used in computer simulations to represent PAs. Many types of PAs can automatically assemble into structures that resemble fibers, and it is this behavior in particular that was studied. By using these models in computer simulations, we are able to see things that cannot be seen in a lab with a microscope or other lab tools. This may help with future efforts to study and design molecules such as PAs which show promise for medical applications like drug delivery. 


\section{Acknowledgements}

I would like to thank the members of the Department of Chemical Engineering at Virginia Tech who specifically contributed to making my time as a graduate student in the department go more smoothly, especially Diane Cannaday (Graduate Coordinator) for all her help and support, Tina Russell (Communications/Program Support), and Dr. David Cox (Professor and Department Head). Their friendliness and willingness to offer assistance and advice were continuous all throughout my time at VT, and for that I am grateful.

I would also like to thank the members of my group for their help and contributions: Dr. Sanket Deshmukh, my advisor, for remaining positive even when the research wasn't developing as desired; Dr. Karteek Bejagam, who provided so much useful help and insight regarding the research and research tools during his time as a post-doctoral researcher in the lab; and Yaxin An, fellow graduate student, who also provided help especially in formulating the research strategy. Thanks also to committee members Dr. John Matson and Dr. Luke Achenie for the parts they played in bringing the research to completion.

This work would never have been possible without the support of my family throughout my entire educational experience. I am forever grateful for them. There were also many individuals in the Blacksburg area who surrounded me with friendship and support while I lived there and made my Virginia experience so much better for it. My thanks to them all. 


\section{Table of Contents}

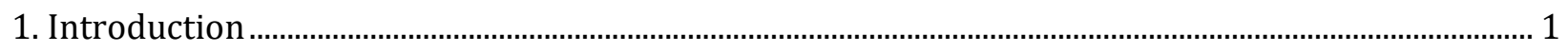

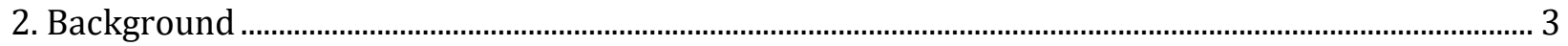

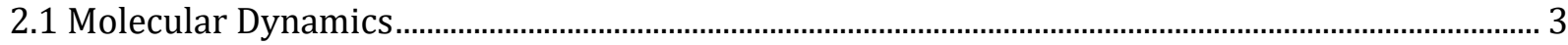

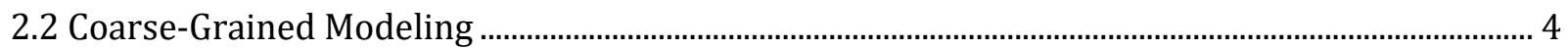

2.3 Amino Acid, Peptide, and Protein Characteristics ......................................................................... 5

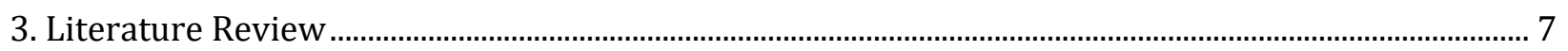

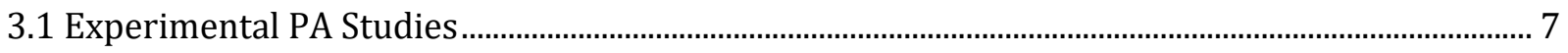

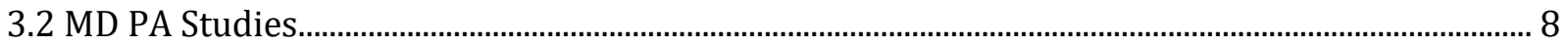

3.3 Methods for Development of CG Amino Acid \& Protein Models...........................................................10

3.4 Notable Protein Models ................................................................................................................................

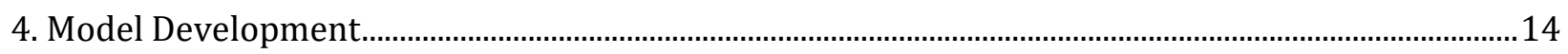

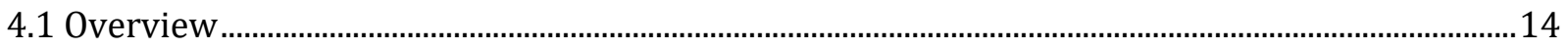

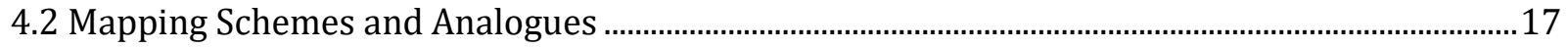

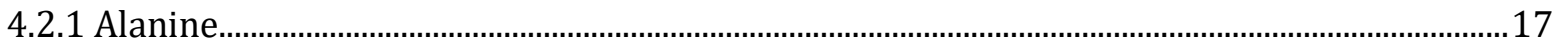

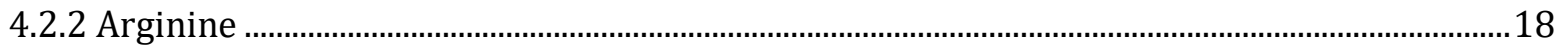

4.2.3 Asparagine ............................................................................................................................................19

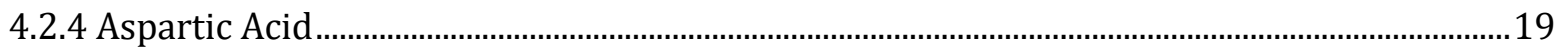

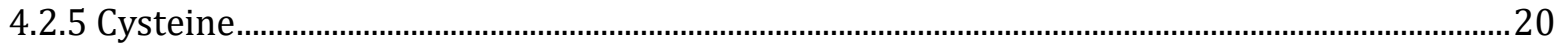

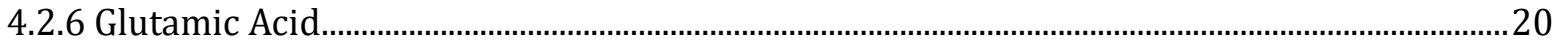

4.2.7 Glutamine

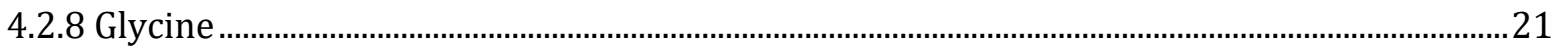

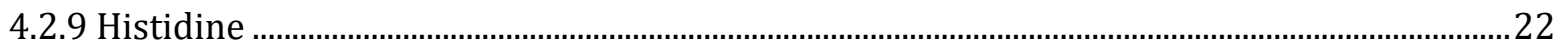

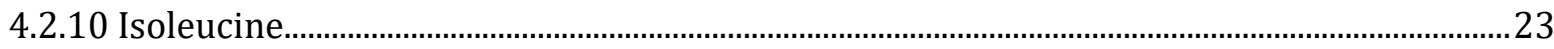

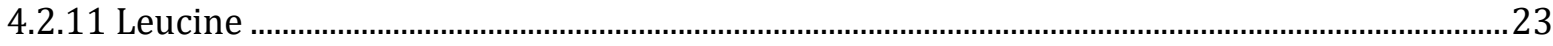

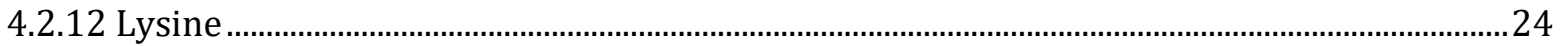

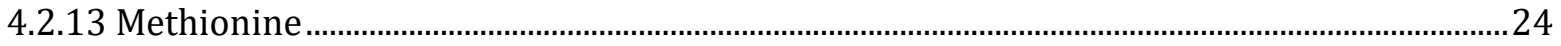

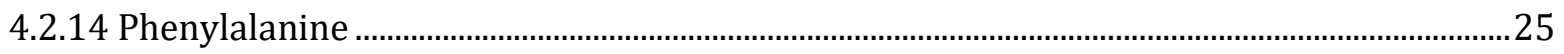

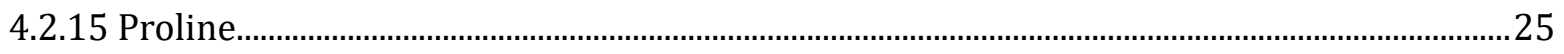

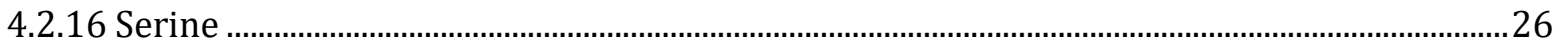

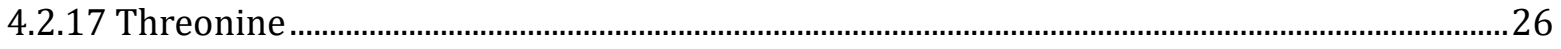

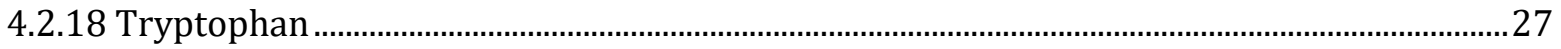




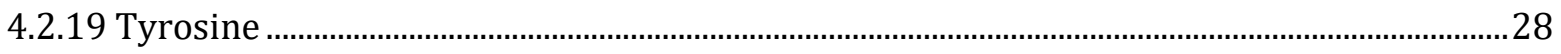

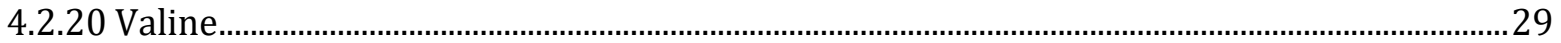

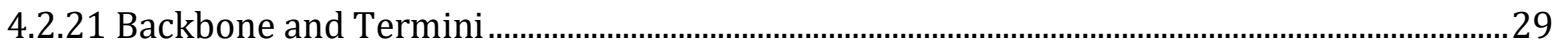

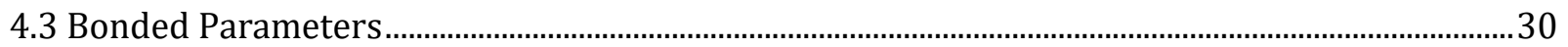

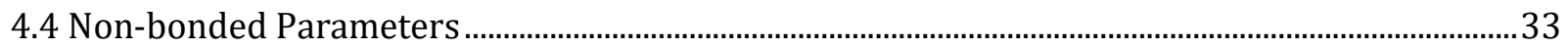

4.5 Calculation of Thermodynamic Properties.................................................................................................. 37

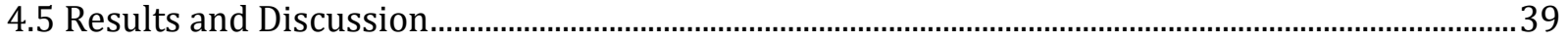

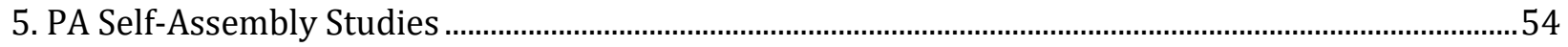

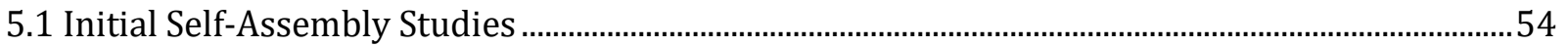

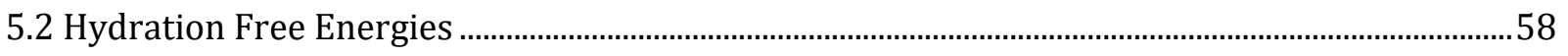

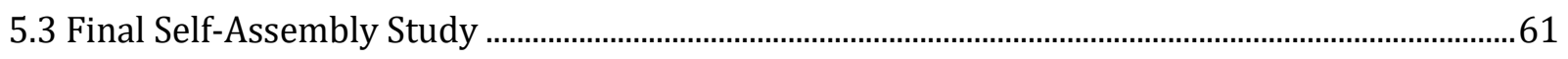

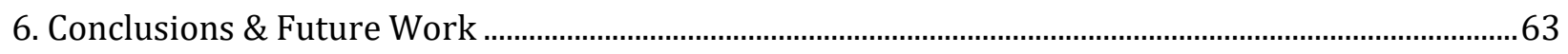

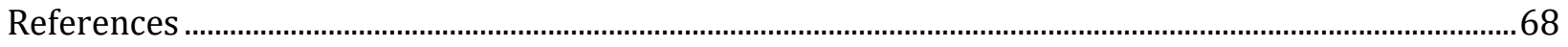

Appendix A: Mapping Schemes............................................................................................................. 74

Appendix B: CG Analogue Parameter Development Results .......................................................................... 81

Appendix C: Peptides and Analogues, Distributions and RDFs ................................................................. 84

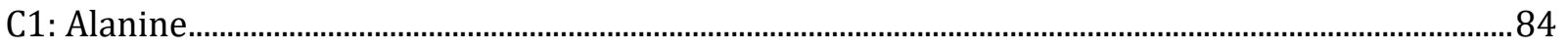

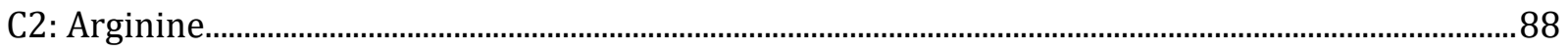

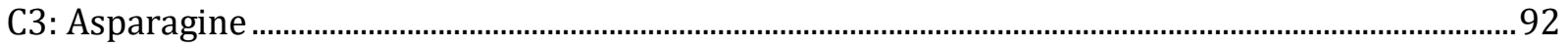

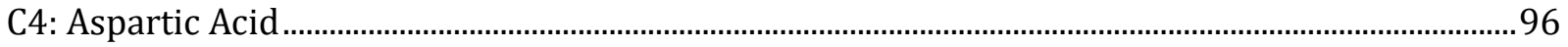

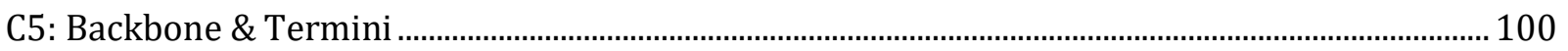

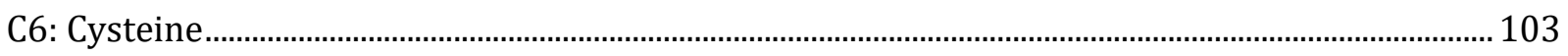

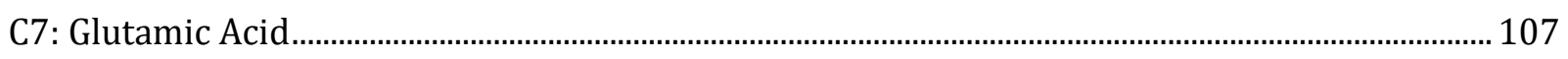

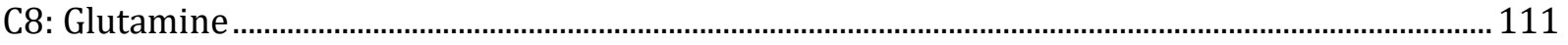

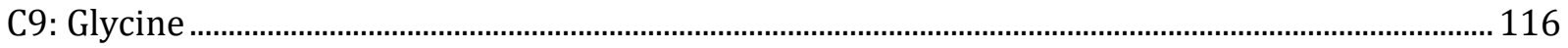

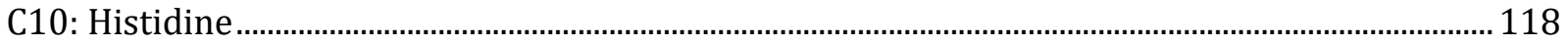

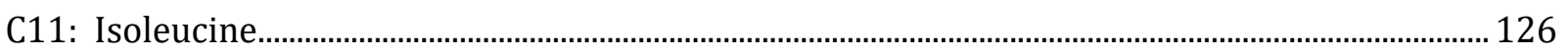

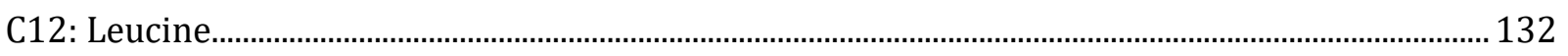

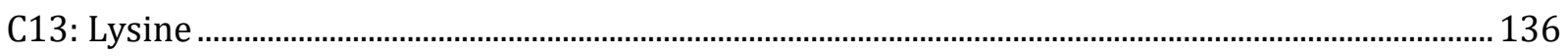

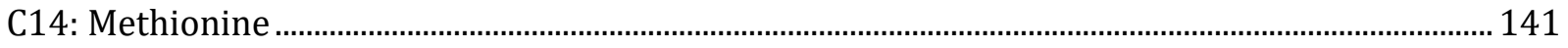

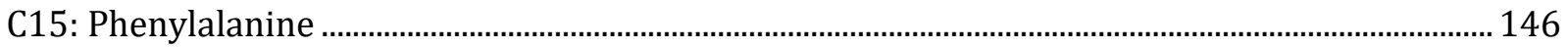

C16: Proline

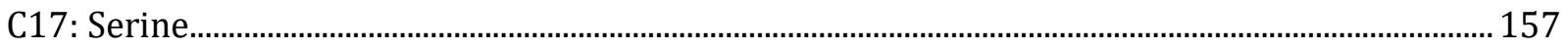




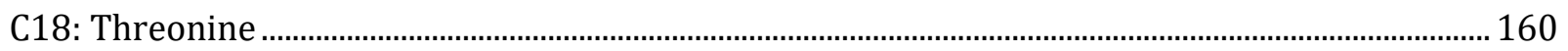

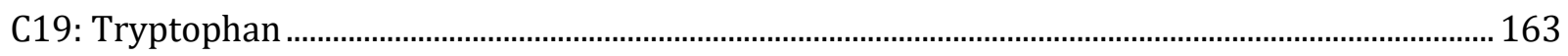

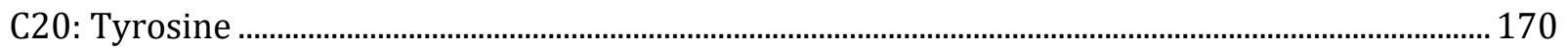

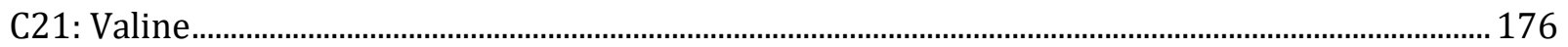

Appendix D: Topology, Parameter, and Configuration Files ...................................................................... 182

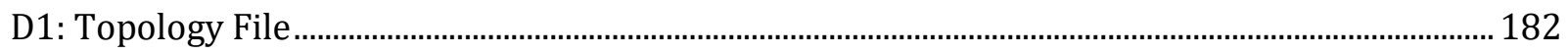

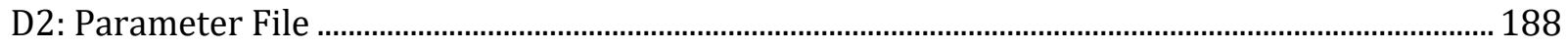

D3: NAMD All-Atom Simulation Configuration File Example....................................................................... 201 


\section{Introduction}

There are twenty common amino acids which are the fundamental structural units of proteins and peptides-molecules that can have important biological functions such as catalyzing biochemical reactions (enzymes), or serving as signaling molecules, transport molecules, or structural molecules, for example. The chemical diversity of the amino acids as "building blocks" in combination with an ability to form secondary structures like pleats ( $\beta$-pleats) and helical twists $(\alpha-$ helices) enables the multiplicity of structures and functions that is necessary for the wider spectrum of forms and operations characteristic of biological systems. Amino acids can also be chemically altered, through synthetic additions for example, for an even wider array of features.

A class of molecules based on amino acids that in recent years has been the subject of much investigation is peptide amphiphiles (PAs). Combining the chemical diversity of the amino acids with a hydrophobic group, such as an alkyl chain, PAs possess the capacity to self-assemble into structures like micelles, sheets, and fibers. ${ }^{1}$ The peptide portion provides potential for biocompatibility and biodegradation, and also bioactivity if created to possess sequence similarity to proteins with biological significance. The amphiphilicity also enables the possibility of encapsulating small hydrophobic drugs. ${ }^{2}$ For these reasons, studies involving PAs have predominantly focused on biomedical applications (e.g. PAs as drug delivery agents and biomaterials for regenerative medicine and tissue engineering, among others). ${ }^{2,3}$ Research such as this is particularly relevant as the world seeks advanced medical therapies that are personalized and specific, with the capability to be engineered for unique situations.

A variety of methods are used to study PAs and their self-assembly. There are experimental, in vitro techniques such as microscopy (e.g. scanning electron microscopy (SEM), transmission electron microscopy (TEM), and atomic force microscopy (AFM)) and spectroscopy (e.g. nuclear magnetic resonance (NMR), transmission infrared spectroscopy (IR), circular 
dichroism (CD), dynamic light scattering, and UV-vis absorption).2,4 While these methodologies are indispensable to the investigation and elucidation of PA self-assembly structures and characteristics, they do not provide us with the ability to directly monitor the self-assembly process on the nanosecond and microsecond timescales. They also do not fully show individual PAs in this process and their interactions with other molecules in their environment like solvent water molecules. Such molecular-level information would be, however, useful for the design of PAs. The computational methodology of molecular dynamics (MD) may help in this respect through modeling, simulation, and visualization of nanometer-level phenomena. In order to conduct MD simulations, appropriate models must first be developed or selected for the system that is to be studied. While models for amino acids and their polymer structures, proteins, exist—as well as models for various other molecules such as hydrocarbons and water-each model has advantages and disadvantages. This thesis provides a discussion of some of the models already in existence that may be used and have been used to study PAs, as well as their limitations, and it also represents work done to design models that may be more appropriate to the study of PA selfassembly.

Background information on MD, MD modeling, and amino acids and peptides/proteins is given in the next few sections, following which an overview of the literature, methodologies, study results, and study conclusions are provided. It is important to note that the development of the amino acid/peptide model that is described in the following pages of this manuscript represents the foundations of such a model, and that frequently, model development is a continuous, ongoing process. Suggestions for future work are also thus provided in the Section 6. 


\section{Background}

\subsection{Molecular Dynamics}

MD is a methodology for modeling molecular systems based on numerically solving Newton's equations of motion. In MD, each atom or particle is represented with mass and radius. Each particle is both acted upon by a potential energy function, also known as a force field, and acts on other particles through that force field. The force fields used for biomolecule and polymer simulations, as shown in equation 1 , often include terms describing the bonded interactions-the bonds, angles, dihedrals, and improper dihedrals (terms 1 through 4 in equation 1)—and the nonbonded interactions as modeled with a Lennard Jones (LJ) potential function (term 5, equation 1) and a term describing electrostatics (term 6, equation 1). Atoms (or particles) in MD are assigned initial positions—often based on molecular geometry as elucidated by experimental analysis of the molecular structure-and initial velocities. In accordance with equations 2-5, the new forces acting upon the particles are calculated and the positions and velocities are updated.

$$
\begin{gathered}
U=\sum k_{b}\left(b_{0}-b\right)^{2}+\sum k_{\theta}\left(\theta_{0}-\theta\right)^{2}+\sum k_{\varphi}\left(\varphi_{0}-\varphi\right)^{2}+\sum k_{\phi}(1+\cos (n \phi-\delta)) \\
+\sum 4 \epsilon_{i j}\left[\left(\frac{\sigma_{i j}}{r_{i j}}\right)^{12}-\left(\frac{\sigma_{i j}}{r_{i j}}\right)^{6}\right]+\sum \frac{q_{i} q_{j}}{4 \pi D r_{i j}}
\end{gathered}
$$

As has been mentioned, MD offers the advantage of enabling the study of systems on much smaller time and length scales than can feasibly be done using experimental, in vitro techniques. This makes it an especially useful technique for studying mechanisms of processes occurring on nano scales such as peptide amphiphile self-assembly.

$$
\begin{gathered}
F=m a \\
F(x)=-\nabla U(x)
\end{gathered}
$$




$$
\begin{gathered}
\frac{d x}{d t}=v \\
\frac{d v}{d t}=\frac{F(x)}{m}
\end{gathered}
$$

\subsection{Coarse-Grained Modeling}

There are two general ways to model molecules for MD simulations: 1) all-atom (AA) modeling, which represents molecules in atom-level detail; and 2) coarse-grained (CG) modeling, which models molecules through the representation of groups of atoms in a molecule as "pseudoatoms" or "beads." 5 In this work, the term "bead" will be used. There are usually multiple choices about which atoms to represent as a single bead. To coarse-grain a benzene molecule, for instance, one could choose to represent the entire molecule as one bead; or it could be modeled with two beads, each containing three of the six carbons in benzene, with these two beads connected by a single bond; or a model of three beads could be created, each containing two carbons, such that the benzene molecule would take the form of a triangle with each bead as a point and the sides the bonds between the beads. These are all possible "mapping schemes" for a CG benzene model, as illustrated in Figure 1. Most commonly in coarse-graining, each bead is represented as a sphere with its center at one of the atoms or at the center of mass of the atoms it represents. The number of heavy atoms (e.g. carbon, nitrogen, oxygen) per bead is represented by the terminology "N:1" (read: $\mathrm{N}$ to 1 ), in which $\mathrm{N}$ is the number of heavy atoms per bead. The hydrogen atoms associated with the heavy atoms are included in the bead. Typically, a bead only represents molecules of a similar type (e.g. four water molecules in one CG water bead) or atoms connected to each other through bonds, ${ }^{5,6}$ as in the benzene example. Modeling molecules this way-by grouping atoms into beads-results in a decrease in the number of degrees of freedom involved in simulating a system containing those molecules. The advantage of that is a corresponding increase in the computational speed for the system which enables one to run a simulation at greater time and length scales than are possible with AA simulations with each atom modeled individually. This 
opens up the possibility of gaining insights into systems and processes that are too large (or long) to be simulated with an AA model or that are too small (or short) to be observed experimentally. CG simulations can span volumes as large as $100 \times 100 \times 100 \mathrm{~nm}^{3}$ that in atomistic detail contain millions of particles, but contain many fewer with coarse-graining. Coarse-graining also makes it possible to study processes that span microseconds to milliseconds in range. Additionally, they can enable high-throughput studies of thousands of parallel simulations, and they may provide insights into the physical nature of a process by revealing which details matter and which do not in comparison with AA simulations. ${ }^{7}$
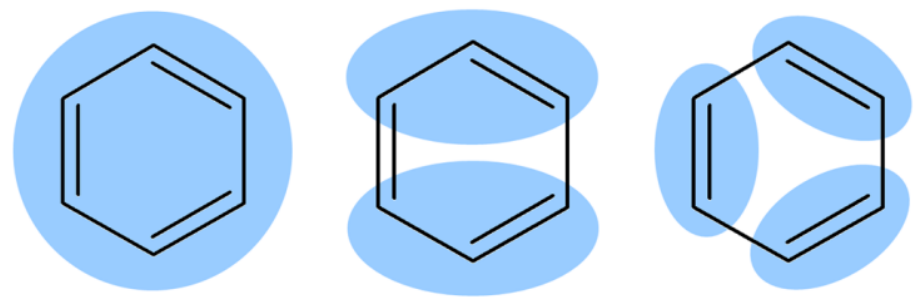

Figure 1: Examples of CG mapping schemes for benzene

When creating a CG model, care must be exercised to capture the essential properties of the molecule(s) insofar as is possible, realistic, and relevant to what is being studied. Coarse-graining smooths out the energy landscape in comparison with AA models, ${ }^{6}$ changes the geometry of the molecule(s), and may result in a variety of other differences depending on the methodology used.

\subsection{Amino Acid, Peptide, and Protein Characteristics}

Amino acids are comprised of three notable chemical groups, two of which are common to all amino acids; these are an amine group and a carboxyl group connected through a carbon atom called the alpha-carbon $\left(\mathrm{C}_{\alpha}\right)$ (shown in Figure 2 in the amino acid Serine as an example). The particular structure of the chemical groups bonded to the alpha-carbon (if any) gives each amino acid its unique identity; this group is known as the side-chain. Only one amino acid, glycine, has no side chain. The rest all exhibit unique groups bonded to the alpha-carbon such that the alphacarbon is chiral, and the standard form found in biological systems is the L-conformation ${ }^{8}$ 
Because of the chemical nature of the amine and carboxyl groups, amino acids can exist in charged and neutral states, and they exist as zwitterions at neutral $\mathrm{pH}$. Amino acids can be classified based on the properties of their side chains: hydrophobicity/hydrophilicity, charge, and polarity. The amino acids lysine, histidine, arginine, aspartic acid, and glutamic acid possess a charge at neutral pH. ${ }^{8}$ A table of all twenty standard amino acids in their neutral forms is given in the appendix (Table A1.1).

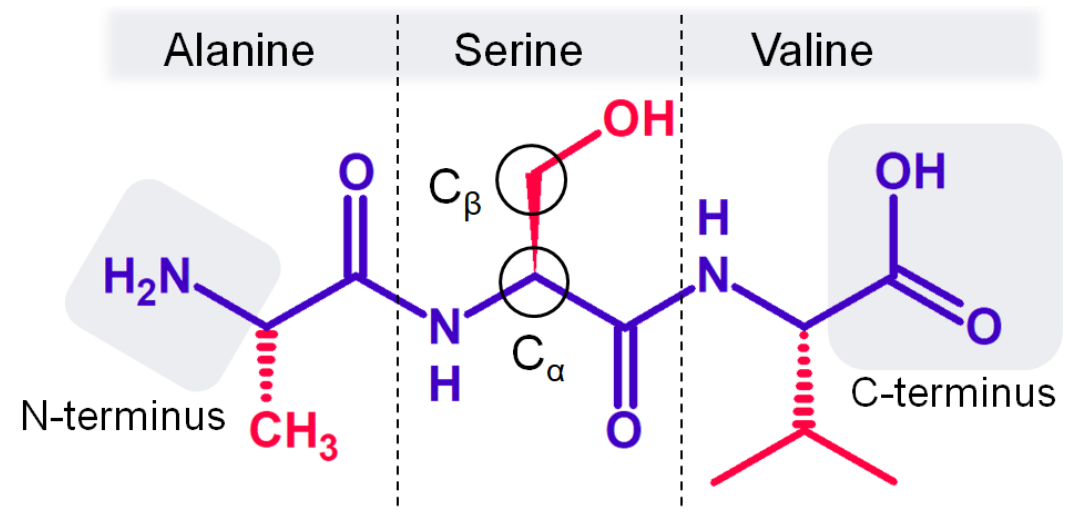

Figure 2: Peptide characteristics shown on an example tripeptide. Blue - peptide backbone; Red - amino acid side chains. Alpha-carbon and Beta-carbon shown on serine as an example.

Amino acids become linked together by the formation of the peptide bond-a condensation reaction in which the nitrogen of the amine group of one amino acid bonds with the carbon of the carboxyl group of another amino acid. ${ }^{8}$ Larger structures that result from this (e.g. proteins and peptide amphiphiles) can be influenced by steric effects of side chains, secondary structures arising from hydrogen bond formation between peptide backbone oxygen and nitrogen atoms, and side chain interactions (e.g. pi-pi stacking). Secondary structures include $\alpha$-helices and $\beta$-sheets which Figure 3 shows.

When developing a CG MD amino acid/protein model, as this work represents, these are some of the characteristics that must be taken into consideration. Section 4 following the literature review will describe the efforts made to capture the desired characteristics. 


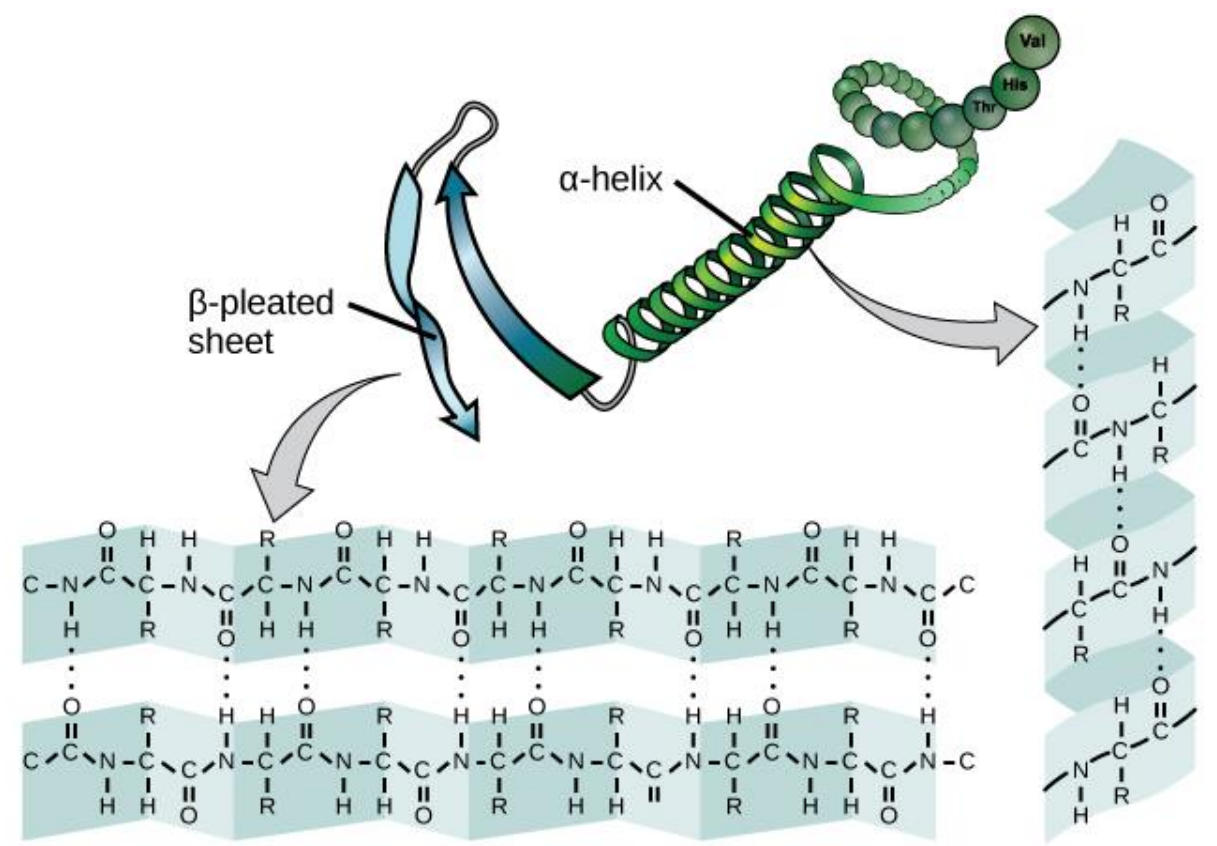

Figure 3: $\beta$-pleated sheet and $\alpha$-helix secondary structures. Hydrogen bonds between peptide backbones are shown with dotted lines. Image from ref 9 , licensed under creative commons.

\section{Literature Review}

\subsection{Experimental PA Studies}

There are many examples in the literature of experimental PA design with self-assembly characteristics and useful applications. A common self-assembled structure is a fiber as shown in Figure 4. In one study, microparticles were synthesized that contained the drug doxorubicin inside a shell of PAs that were functionalized to target the folate receptor. A 60-fold higher cytotoxicity was reported of the microparticles against a particular type of breast cancer cells, MDA-MB-231, compared with non-targeting particles and commercial doxorubicin. ${ }^{10}$ In another study, selfassembly was demonstrated of a PA nanofibrous system with an antisense oligonucleotide (a potential therapeutic for several disorders) that exhibited enhanced cellular uptake and activity as compared with the oligonucleotide delivered without the peptide amphiphile. ${ }^{11}$ The Stupp lab has investigated PAs for neural regeneration. They incorporated the laminin-derived IKVAV amino acid sequence into PAs and showed rapid, selective differentiation of neural progenitor cells into 
neurons when cultured within the IKVAV PA. ${ }^{12}$ They then used the material to treat spinal cord injury and demonstrated a reduction in cell death at the site of the injury as well as an increase in oligodendroglia (cells that form the myelin sheath) and regeneration of neurons. ${ }^{13}$ More recently, the Matson lab designed hydrogels of functionalized self-assembling aromatic PAs for controlled release of $\mathrm{H}_{2} \mathrm{~S}$ with potential biological applications. ${ }^{14}$ This is representative of the leading edge of research in drug delivery: to create biocompatible delivery systems that enable localized drug delivery and controlled release rates, and which undergo biodegradation in the process of-or shortly following—drug release. The PA-based hydrogels created by the Matson group have potential in all these areas.

PAs may also be used for photochemical/photophysical and electronic applications such as light-harvesting complexes. For example, Matsui and MacCuspie were able to fabricate metalloporphyrin nanotubes by coating PA nanotubes with the metalloporphyrin protoporphyrin IX Zn(II). ${ }^{15}$ Fry, Stupp, and coworkers also experimented with peptide amphiphiles and protoporphyrin IX Zn(II). They synthesized self-assembled PA metalloporphyrin arrays that showed strong exciton interactions influenced by the peptide. ${ }^{16}$ Garifullin, et al., reported the encapsulation of a derivative of zinc phthalocyanine within self-assembled peptide amphiphile nanofibers. The resulting complexes showed very fast intermolecular energy transfer, demonstrating the potential of PAs for the fabrication of supramolecular organic electronic devices. ${ }^{17}$ Thus it can be seen that a substantial amount of research has shown PAs to be promising candidates in the quest for improved and enhanced therapies and technologies.

\subsection{PA Studies}

Considering the diversity of characteristics and form as well as the potential applications that PAs have, research efforts have been made in the area of mechanisms of PA self-assembly into supramolecular structures such as fibers. In MD studies, both AA models (e.g. CHARMM,18 Amber, ${ }^{19}$ $\mathrm{OPLS}^{20}$ ) and CG models (e.g. Martini, ${ }^{21} \mathrm{PRME}^{22}$ and $\mathrm{SDK}^{23}$ ) have been used. Experimental and MD 
studies have both shown that for most PA fibers, their assembly occurs first through the formation of micelles followed by the merging of micelles to form short cylindrical structures followed by elongation into fibers through further merges. ${ }^{24-26}$ Early experimental and MD studies indicated that hydrophobic interactions of the alkyl tails of PAs and the formation of $\beta$-sheets between the peptide portions of PAs are integral to the formation of fibers. ${ }^{27}$ A CG MD study performed by Velichko, Stupp, and Olvera de la Cruz showed that under conditions of pure hydrophobic interactions without hydrogen bonding, micelles will form. ${ }^{28}$ When the hydrophobic interactions were turned off and hydrogen bonding interactions were turned on, the PAs organized into onedimensional $\beta$-sheets. Fibers were only observed when both significant hydrophobic attraction and hydrogen bonding resulting in $\beta$-sheet formation were at play. Later studies, however, cast doubt on the importance or even necessity of $\beta$-sheet formation for fiber formation, showing instead that $\beta$-sheet content can be negligible in PA fibers and that van der Waals forces-and in some cases electrostatic interactions - may have a much more significant role in fiber formation, along with hydrophobic interactions..$^{24,29,30}$ Research has also suggested that water ordering near the comparatively hydrophilic peptide portion of a PA provides structure to the PAs and thereby facilitates fiber formation-insights gleaned from combined AA and CG MD studies supported by experimental validation. ${ }^{4}$ Altogether, the particular stable form (e.g. micelle, fiber, tube) of different PA self-assembled structures results from the particular strengths of and interplay between van der Waals forces, hydrogen bonding, and electrostatic interactions among the chemical constituents of the PAs and their surroundings. ${ }^{31,32}$ While insights thus have been gained through the use of MD, the particular models implemented in the study of amino acid based molecules do have various limitations. In order to understand some of the strengths and weaknesses of these models, however, there must first be an understanding of MD and MD model development. Thus the next few sections provide an overview of these topics, as well as some details on amino acids and their 
characteristics which must be considered when modeling them, and then will be described some of the models that may be used for PAs.

\subsection{Methods for Development of CG Amino Acid \& Protein Models}

Several methods have been used to develop amino acid/protein models. They generally fall into two categories based on the mathematical formulas used to define the potentials: 1) physicsbased - in which the CG potential is described by bonded terms relating bond deformation and angles, as well as non-bonded terms describing Lennard-Jones interactions (dispersion and repulsion effects) and electrostatic interactions; and 2) knowledge-based - an approach in which the CG potentials are determined by statistical analysis of known protein structures and defined by statistical models. Physics-based methods can be further subdivided into "bottom-up" and "topdown" approaches. The former is one in which AA models are used as the basis by which CG potentials are derived; while the latter is thermodynamics-based, in which the CG potentials are derived by reproducing (as closely as possible) key experimental data, especially thermodynamic properties such as surface tension, free energy of solvation, and density.6,7

With respect to AA-based CG force field development, there are three primary techniques that have been employed: the inverse Monte Carlo (IMC) method, iterative Boltzmann inversion (IBI), and force matching (FM), with the latter two being the most popular. ${ }^{7}$ With the IBI method, the goal is to match the CG bead radial distribution function (RDF) with that of its AA counterpart using a CG pair potential. As its name suggests, it is an iterative process that proceeds until there is a convergence of the potential yielding the correct RDF. However, a particular RDF may be reproduced by many different pair potentials such that the solution is not unique. Yet the IBI method has been very popular because of its simplicity and quickness of convergence. ${ }^{6}$ The IMC method also attempts to match the CG RDF with the AA RDF, and it too is an iterative process. It differs from IBI in that it handles correlations between CG force field parameters in an explicit way—such that their values are updated in an interdependent way, whereas in the IBI method the 
updates are independent—and with each iteration a set of linear equations is solved to find a better approximation for the force field parameters.6,33 Force matching, on the other hand, attempts to match CG pairwise forces to AA forces calculated based on a set of reference conformations. A later development used AA molecular dynamics (MD) trajectories as the reference. This process also may be iterative. ${ }^{6}$

The AA-based, physics-based approaches are more likely to capture some of the fine details of the system while the top-down approach of using experimental thermodynamic data is more likely to provide CG potentials that are transferable to other systems or states. ${ }^{7}$ A protein-structure statistically-derived method can work well for computational biology projects because CG forcefields derived in this manner are simple and efficient. Yet their weakness is also in a lack of transferability; as Ingólfsson et al. describe it, “While the force field derived for single domain globular proteins will work well for a vast majority of single protein and peptide structures, the interaction between independent domains, interactions between proteins and nucleic acids, etc. require derivation of a new component of knowledge-based force fields."7

Another method of CG model development, as mentioned, is to optimize model parameters to reproduce experimental, thermodynamic properties. Examples of models that utilize this method include the Martini model, in which partitioning free energies of amino acid side chains between water and oil (cyclohexane and butane) were used to develop non-bonded parameters; ${ }^{21}$ and the Shinoda-Devane-Klein (SDK) model which used experimental values of density and surface tension for non-bonded amino acid/protein parameters of side chain analogues. ${ }^{23}$ Both of these models, along with some others, will be further described in the following section.

\subsection{Notable Protein Models}

Amino acid CG models have predominantly been developed for examining the folding, characteristics, and behavior of proteins in large systems. ${ }^{6}$ Because such systems consist of a large number of particles, it is quite common to incorporate solvent effects into the model so that solvent 
molecules are not simulated-thereby decreasing the total number of degrees of freedom and significantly speeding up the simulation. In those cases, the model is an implicit solvent model, whereas a model that requires explicit representation of solvent molecules is called an explicit solvent model. As can be seen in Table 1, many CG protein models are implicit solvent models: AWSEM (associative memory, water mediated, structure and energy model) ${ }^{34}$; Bereau \& Deserno 35 ; CABS (c-alpha, c-beta, side-chain) ${ }^{36}$; Hills, Lu, \& Voth ${ }^{37}$; OPEP (optimized potential for efficient protein structure prediction) ${ }^{38}$; PaLaCe ${ }^{39}$; PRIME (protein intermediate resolution model)22; PRIMO (protein intermediate model) ${ }^{40}$; Rosetta ${ }^{41}$; and UNRES (united residue) ${ }^{42}$ models. Many of these models place a greater emphasis on the protein backbone than on the side chains due to the influence of the backbone on secondary structure characteristics and protein folding. Exceptions are the Martini21; PRIMO; and Hills, Lu, and Voth models, which allow a higher resolution for the side chains than many of the other models. Of the explicit solvent models, the Martini model is certainly more widely used than the SCORPION model ${ }^{43}$, especially because the model has already been well developed for other types of molecules—such as lipids ${ }^{44}$ and DNA ${ }^{45}$ - which is of use when modeling proteins in biological systems.

The Shinoda-Devane-Klein (SDK) model23 is another CG force field that can be used for protein and peptide simulations, yet only non-bonded parameters were developed for the model and not bonded parameters (only generic bonded parameters were used in the parameterization of the model). Those who wish to use the SDK model thus may need to develop their own bonded parameters as in reference ${ }^{46}$. Regarding the non-bonded interactions, the softer LJ (9-6) potential form was used rather than the LJ (12-6) form shown in equation 1 . Additionally, the backbone was modeled in a somewhat crude way by simply using the Asparagine and Glutamine side chain beads for the standard and Alanine backbone beads. ${ }^{23}$ However, there is also an SDK CG water model that can be used in conjunction with the SDK protein model, in which parameters were developed for a CG water bead comprised of three water molecules. ${ }^{46}$ 
Table 1: Select CG Protein Models

\begin{tabular}{|c|c|c|c|c|}
\hline Model & Solvent & $\begin{array}{l}\text { Backbone } \\
\text { Beads }\end{array}$ & $\begin{array}{l}\text { Side Chain } \\
\text { Beads }\end{array}$ & Development \\
\hline AWSEM & implicit & $2\left(\mathrm{C}_{\alpha}, \mathrm{O}\right)$ & $1\left(\mathrm{C}_{\beta}\right)$ & $\begin{array}{l}\text { mixed: knowledge-based and } \\
\text { physics-based }\end{array}$ \\
\hline Bereau \& Deserno & implicit & 3 & $1\left(\mathrm{C}_{\beta}\right)$ & knowledge-based \\
\hline CABS & implicit & 3 & 1 & knowledge-based \\
\hline Hills, Lu, \& Voth & implicit & $1\left(\mathrm{C}_{\alpha}\right)$ & up to 4 & $\begin{array}{l}\text { physics-based, force- } \\
\text { matching }\end{array}$ \\
\hline Martini & explicit & 1 & up to 4 & $\begin{array}{c}\text { physics-based, } \\
\text { thermodynamics-based }\end{array}$ \\
\hline OРЕР & implicit & 5 & 1 (proline: 3 ) & $\begin{array}{l}\text { mixed: physics-based and } \\
\text { knowledge-based }\end{array}$ \\
\hline PaLaCe & implicit & 3 & $1-2$ & $\begin{array}{l}\text { physics-based, iterative } \\
\text { Boltzmann inversion }\end{array}$ \\
\hline PRIME & implicit & 3 & 1 & knowledge-based \\
\hline PRIMO & implicit & 3 & up to 5 & physics-based \\
\hline Rosetta & implicit & $\begin{array}{l}\text { all backbone } \\
\text { atoms }\end{array}$ & $1-2$ & $\begin{array}{l}\text { mixed: knowledge-based and } \\
\text { physics-based }\end{array}$ \\
\hline SCORPION & explicit & 1 & $1-2$ & $\begin{array}{l}\text { physics-based, force- } \\
\text { matching }\end{array}$ \\
\hline UNRES & implicit & 2 & 1 & physics-based \\
\hline
\end{tabular}

It has already been shown that water plays a critical role in the self-assembly of PAs, ${ }^{4}$ and for that reason it seems best to employ explicit solvent in PA self-assembly simulations. As noted already, the majority of protein models developed have been developed with implicit solvent parameters. SCORPION, an explicit solvent CG protein model, only models amino acid structures with one backbone bead per amino acid residue (the monomeric unit), and side chains are modeled with at most two beads for a given amino acid. This is one of the lower resolution options, whereas 
a more intermediate resolution model of both the side chains and backbone may be more effective at closely capturing the behavior and interactions of both during PA self-assembly. Additionally, the SCORPION model utilizes an elastic network rather than explicit bonded terms for the bonded interactions. ${ }^{43}$ The Martini model has a higher resolution for the side chains with up to four beads for a given amino acid. However, the backbone is modeled with just one bead per residue, and when using the Martini model, it is required that one specify the type of backbone bead-whether it is in an alpha helix or beta sheet or free in solution, for example. Furthermore, the Martini CG water model shows freezing behavior between 280-300 K which the developers corrected by introducing antifreeze particles along with the water to reproduce liquid behavior in that temperature range. ${ }^{44}$ A set of CG water models that do not have this limitation has been recently developed in our group by Bejagam et al. ${ }^{33}$

While any of the protein models discussed in the preceding paragraphs could be chosen for studying PAs, it may be that none of them captures PA-water interactions accurately, considering some of the limitations addressed. If that is the case, then it is possible that the self-assembly pathways demonstrated from the use of those models may be wrong. Yet a thorough knowledge of the actual steps involved in the process of self-assembly would be advantageous for designing PAs and controlling the self-assembly process. Therefore we considered the development of new CG amino acid models a worthy endeavor-models whose interaction parameters could be tuned with respect to the aforementioned water model developed in our group. ${ }^{33}$

\section{Model Development}

\subsection{Overview}

In addition to utilizing the water models developed by Bejagam, et al. ${ }^{33}$ in order to create amino acid models for use with explicit solvent, we were interested in using other CG models that members of our lab had recently developed: CG models for hydrocarbons ${ }^{47,48}$ and benzene. ${ }^{33}$ These 
could be used for modeling hydrocarbon tails of PAs and parts of amino acids as well. We determined to create an intermediate-resolution model of both the amino acid side chains and the peptide backbone so as to more closely capture the behavior of both during PA self-assembly. The peptide backbone would be parameterized such that it would not be necessary to set a predetermined secondary structure, unlike in the Martini model. ${ }^{21}$ Additionally, a 12-6 LJ functional form was chosen so as to harmonize with the water, hydrocarbon, and benzene models since those were developed with the same LJ functional form.

Our mapping schemes would be 2:1 where possible, and only larger (e.g. 3:1 or 4:1) when constrained by the atomic bonding pattern within the molecule or in the event of other constraints necessary for creating a stable CG model. A 2:1 mapping scheme would allow for greater accuracy as compared with beads containing larger numbers of heavy atoms per bead, while simultaneously offering an advantage in a decrease in the number of degrees of freedom which increases computational speed as compared with AA models as previously described. The maximum number of heavy atoms represented in a given bead in the models developed is four ( $4: 1$ beads), as can be seen in the mapping schemes given in Table A1.1 in the appendix. The overall ring structures of Phenylalanine, Tyrosine, Tryptophan, and Histidine were preserved. The centers of the beads are located at the center of mass of the atoms they represent. The mapping schemes that were developed also provide for transferability across amino acids where possible, such that in some cases the same bead type could be used in more than one amino acid (e.g. C2M in methionine, glutamine, and glutamic acid).

We determined to develop a physics-based model as opposed to a knowledge-based model since the latter is derived from statistical analysis of large amino acid assemblies in the form of proteins and protein complexes, neither of which represents the target application for our model. We also determined that the base, initial model would only possess neutral beads corresponding to neutral (uncharged) amino acid side chains and termini. The terms that our model would need to 
account for are thus all of those given in equation 1 with the exception of the fifth term representing electrostatic interactions which is important for force fields (e.g. AA force fields) that possess particles with full or partial charges. We also chose a hybrid method for parameterizing our model that utilizes AA distributions for developing the CG bonded parameters and experimental thermodynamic data for developing the non-bonded parameters of the CG force field. This methodology was successfully used in our group to develop the hydrocarbon models. ${ }^{47}$ The bonded parameters we could obtain by mapping distributions from AA molecular dynamics simulation trajectories to CG distributions. The non-bonded parameters were determined in a manner similar to that used in the SDK model development, in which the non-bonded parameters or side chain beads were optimized to reproduce thermodynamic properties of amino acid side chain analogues.23 Side chain analogues are molecules that closely approximate the structure of the amino acid side chains. For example, the side chain analogue of neutral Lysine is N-butylamine as is shown in Figure 16 on page 26. While the SDK model was reported to have performed well in predicting the native structures of a set of proteins, bonded parameters were not specifically developed for the model, and beads that represented the backbone were patterned off of the Asparagine and Glutamine side chains ${ }^{23}$ as previously mentioned. Additionally, while the SDK model was parameterized using only experimental values for density and surface tension, we included a third optimization experimental value, heat of vaporization, when such information was available for an analogue. Including heat of vaporization in the optimization process would help ensure that the non-bonded parameters were really ideally optimized to reproduce the appropriate thermodynamics of the chemical constituents of the amino acids.

Following development of the bonded and non-bonded parameters, we would be able to run CG PA simulations to test for self-assembly and the appropriateness of the parameters, and to determine what subsequent steps should be taken in the model parameterization. Tuning the 
interaction parameters with the water model also could be evaluated. Obtaining hydration free energies for amino acid analogues was also used for that purpose.

\subsection{Mapping Schemes and Analogues}

Determining the mapping scheme is one of the first steps in CG model development. In this section, the mapping schemes for the amino acids will be shown. The choice of analogues used to represent the amino acid side chains, backbone beads, and termini beads will be discussed. Any particularities for a given amino acid or analogue will also be conveyed. More details of the mapping schemes are given in Table A1.1 (for the amino acids) and Table A1.2 (for the analogues) in the appendix.

\subsubsection{Alanine}

(a)

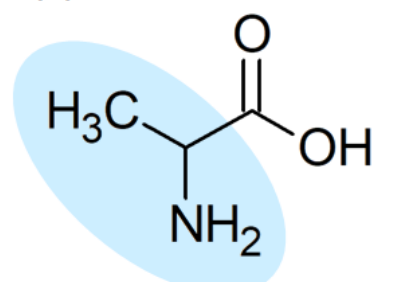

(b)

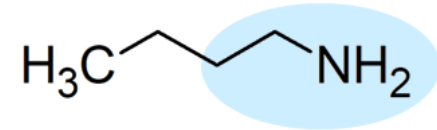

Figure 5: (a) Alanine with mapping scheme and (b) N-butylamine

Alanine is the amino acid with the smallest side chain-just a methyl group. This made it necessary to model the alanine backbone differently than the standard backbone associated with most of the other amino acids, with the side chain grouped in with the alpha carbon and nitrogen, as shown in Figure 5. To have modeled the side chain separately would have resulted in a bead so lightweight that it would have been unstable at larger time steps than are possible when implementing a 2:1 or greater ratio mapping scheme. A lower mass leads to a greater vibrational frequency in connection with any beads it is bonded to, and this correlates with a smaller time step required for stability. ${ }^{49} \mathrm{~N}$-butylamine was chosen for parameterizing the alanine beads because of 
its structural similarity, with both the backbone Alanine bead ( $\mathrm{AB}$ ) and the N-terminal alanine bead (ANT) modeled with the same non-bonded parameters.

\subsubsection{Arginine}

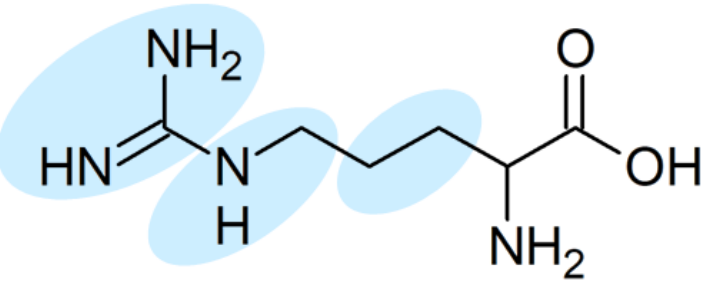

Figure 6: Arginine with side chain mapping scheme

The structure of the arginine side chain is the most unique as compared with the other amino acid side chains. The closest analogue to the nitrogenous end group is guanidine, for which not a large quantity of experimental thermodynamic data are available. The pKa of this end group is $13.8,^{50}$ meaning it is almost never in neutral form, and almost always it is protonated. DeVane, et al., during the creation of the SDK model, used experimental data from guanidinium salt solutions to parameterize the charged end group of arginine. ${ }^{23}$ Yet since the base model development described in this thesis is only of neutral species, we modeled a neutral form of arginine as well. We used three beads to describe the side chain, as shown in Figure 6. For the outermost bead in the side chain-which represents two nitrogen atoms and one carbon with associated hydrogens-we used an AA model of the arginine side chain and ran simulations to calculate values for the density, heat of vaporization, and surface tension at $298 \mathrm{~K}$ for optimizing the non-bonded parameters. Because the CHARMM AA force field does not include a model of a neutral arginine, we obtained partial charges for the atomic model from Gaussian ${ }^{51}$, an ab initio quantum chemistry software package that can be used for predicting many properties of molecules, including atomic charges. Some of these charges were modified so as to harmonize better with the CHARMM values for the side-chain atoms and assign no difference between hydrogens bonded to the same carbon atom, as shown in Table A4.1 in the appendix which provides the atom names (as given in the CHARMM files), the 
Gaussian charges, the CHARMM charges, and the charges that were assigned. Regarding the other beads in the CG model, the middle side chain bead was modeled with the same non-bonded parameters as the NC (nitrogen plus $\mathrm{C}_{\alpha}$ ) bead of the backbone, and the inner side chain bead was modeled with the same non-bonded parameters as those of the two-carbon C2M bead previously developed by a member of the group from modeling hydrocarbons. ${ }^{47}$

\subsubsection{Asparagine}

(a)

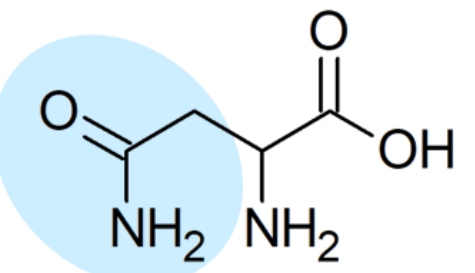

(b)

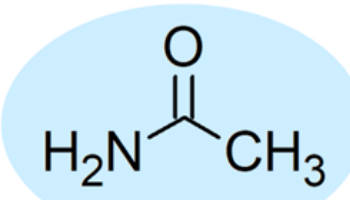

Figure 7: (a) Asparagine with side chain mapping scheme, and (b) acetamide

Because of the structure of the asparagine side chain, it was necessary to model the entire side chain as one bead as shown in Figure 7(a) above. Experimental values of acetamide were used for optimizing the non-bonded parameters. However, because the melting point of acetamide occurs at $81^{\circ} \mathrm{C}(354 \mathrm{~K}), 5^{52}$ all simulations were run at $358 \mathrm{~K}$ at which temperature the molecule is in liquid form. This was for the calculations performed in the optimization of the non-bonded parameters, as is described in Section 4.4 .

\subsubsection{Aspartic Acid}

(a)

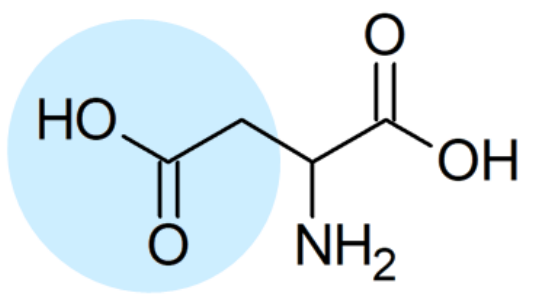

(b)

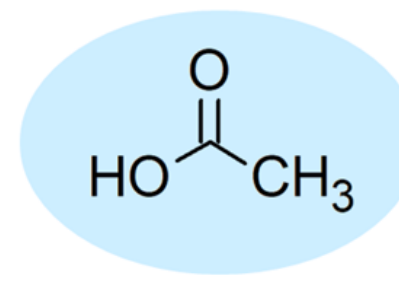

Figure 8: (a) Aspartic acid with side chain mapping scheme and (b) acetic acid 
Like asparagine, the aspartic acid side chain was modeled as one bead (Figure 8(a)) because of the bonding pattern between the side chain atoms. Acetic acid experimental values were used for optimizing the non-bonded parameters.

\subsubsection{Cysteine}

(a)

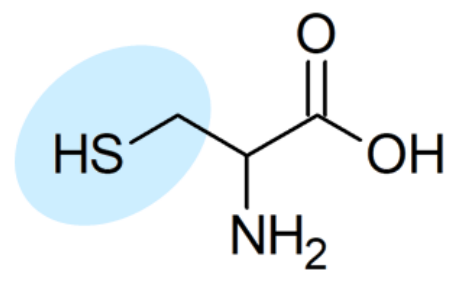

(b)

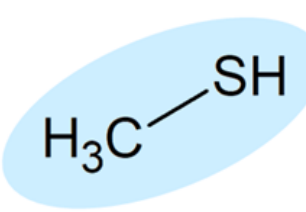

Figure 9: (a) Cysteine with side chain mapping scheme and (b) methanethiol

The analogue for the cysteine side chain is methanethiol (methyl mercaptan); its similarity to the cysteine side chain is shown in Figure 9 above. Its boiling point is $6^{\circ} \mathrm{C}(279 \mathrm{~K})^{52}$ so all simulations were run at $278 \mathrm{~K}$. The value for the heat of vaporization used was that at the boiling point, which is given in Section 4.5, as a close approximation to the value at $278 \mathrm{~K}$ since a value was not found for the slightly lower temperature.

\subsubsection{Glutamic Acid}

(a)

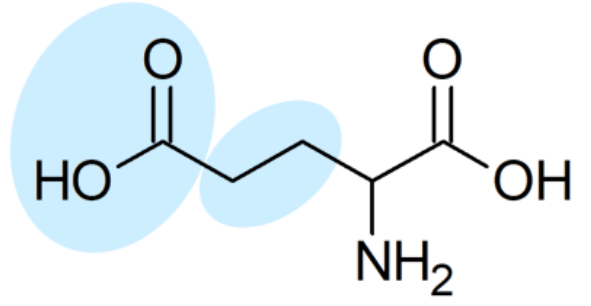

(b)

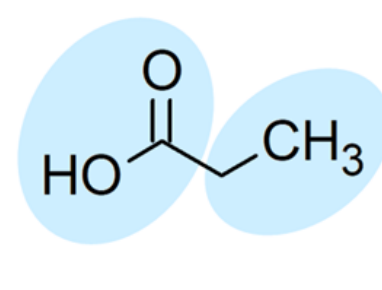

Figure 10: (a) Glutamic acid with side chain mapping scheme and (b) propionic acid

Two beads were used to model the glutamic acid side chain (Figure 10(a)). Non-bonded parameters for the outer bead-representing the carboxyl group of the side chain-had already been obtained by previous work done in the group (unpublished results) from optimization with 
respect to propionic acid experimental values. The inner bead was modeled with the same parameters as the $\mathrm{C} 2 \mathrm{M}$ bead obtained in previous work. ${ }^{47}$

\subsubsection{Glutamine}

(a)

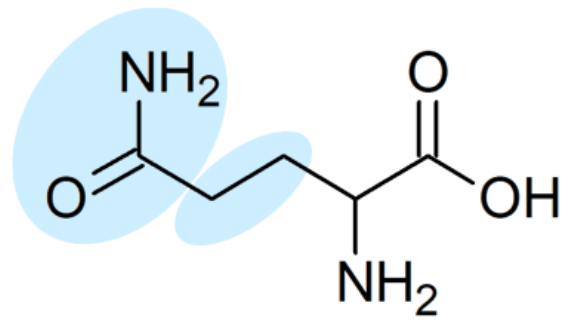

(b)

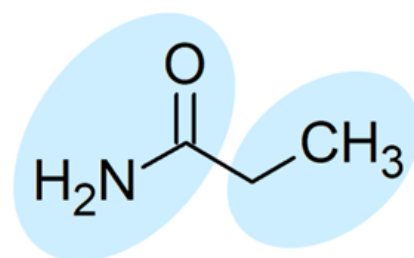

Figure 11: (a) Glutamine with side chain mapping scheme and (b) propionamide

Propionamide was used as the analogue for glutamine as it represents the side chain very accurately as can be seen in Figure 11 . Its melting point is $81^{\circ} \mathrm{C}(354 \mathrm{~K}), 52$ so all simulations were run at $356 \mathrm{~K}$ to target experimental thermodynamic properties of the liquid phase for optimizing the CG force field parameters. The inner side chain bead was assigned the C2M type as in the case of the inner side chain bead of glutamic acid.

\subsubsection{Glycine}

(a)

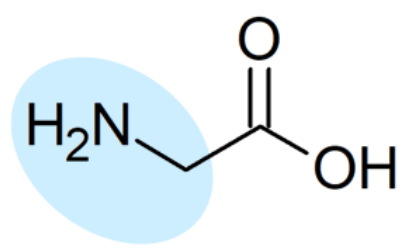

(b)

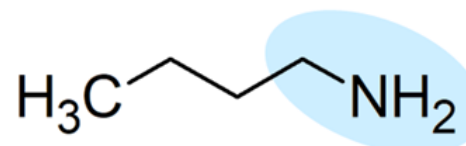

Figure 12: (a) Glycine with mapping scheme and (b) N-butylamine.

Glycine is the only amino acid that does not have a side chain. Its N-terminal $\mathrm{C}_{\alpha}$-amine group was modeled the same as any other $\mathrm{N}$-terminal group, and its backbone $\mathrm{C}_{\alpha}$-amine group was modeled the same as the standard backbone $\mathrm{C}_{\alpha}$-amine group, the only difference in both cases being an extra hydrogen as compared with the other N-terminal and backbone groups. The standard Nterminal analogue is N-butylamine which is shown in Figure 12 above to be a good analogue for the glycine N-terminal bead. However, analysis of the AA CG-mapped distributions would indicate 
whether the bonded parameters should be modeled the same as for the other standard amino acids or differently.

\subsubsection{Histidine}

(a)

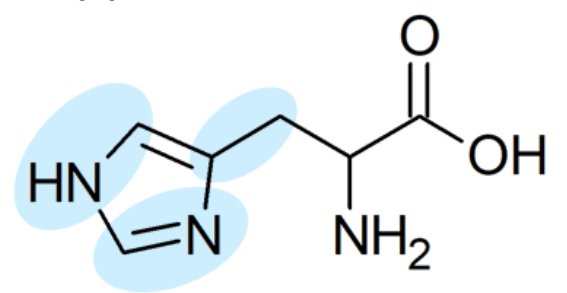

(c)

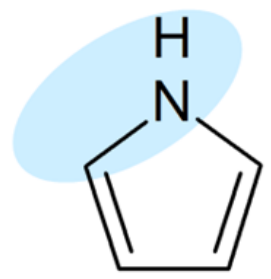

(b)<smiles>c1ccncc1</smiles>

(d)

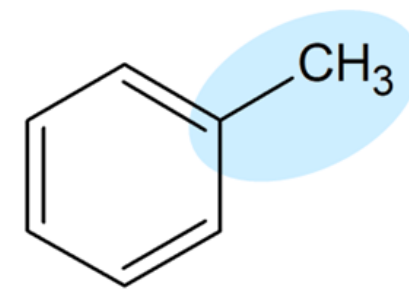

Figure 13: (a) Histidine with side chain mapping scheme, and side chain analogues (b) pyridine, (c) pyrrole, and (d) toluene.

The analogue of the histidine side chain is 4-methylimidazole. Because experimental thermodynamic properties for the analogue are lacking in its liquid phase, we used pyridine, pyrrole, and toluene for optimizing the parameters of the three histidine side-chain beads. The similarities of some of the constituents of these analogues to the histidine side chain mapped constituents can be seen in Figure 13. The $r_{\text {min }}$ values for the analogue and amino acid beads were compared to check for similarity as a validation (or contradiction) of the appropriateness of those analogues for parameterizing the histidine beads. 


\subsubsection{Isoleucine}

(a)

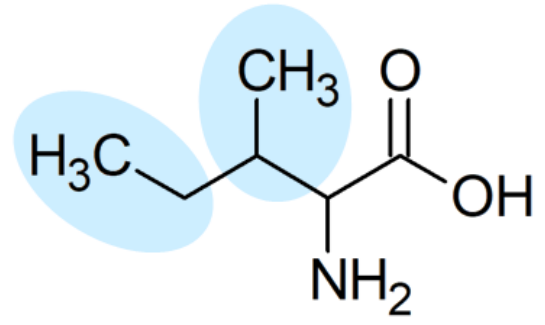

(b)

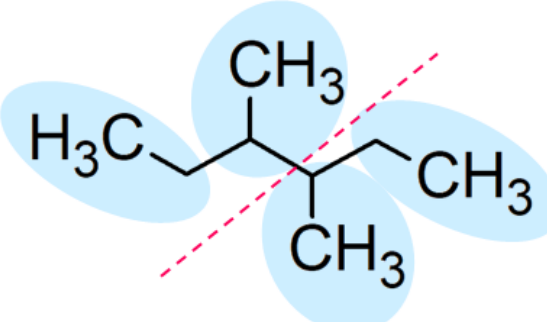

Figure 14: (a) Isoleucine with side chain mapping scheme and (b) 3,4-dimethylhexane. Dashed line shows that 2,5-dimethylhexane is a symmetric analogue of the isoleucine side chain.

Butane is a close analogue of the isoleucine side chain, but it exists as a gas at room temperature, and its condensation point is $-0.5^{\circ} \mathrm{C} .5^{2}$ Therefore, we chose 3,4 -dimethylhexanewhich is a liquid at room temperature ${ }^{52}$-for optimizing the non-bonded parameters of the isoleucine side chain. This is the same approach as that taken in the development of the SDK model ${ }^{23}$ We gave the outermost side chain bead the same parameters as the C2E bead in the hydrocarbon optimized previously by An, et. al. ${ }^{47}$ Yet because of the slight difference between the inner side chain bead and the outer side chain bead, as shown in Figure 14, we decided to model the inner side chain bead as different from the outer side chain bead.

\subsubsection{Leucine}

(a)

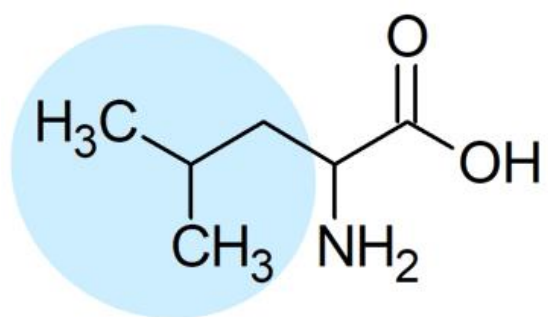

(b)

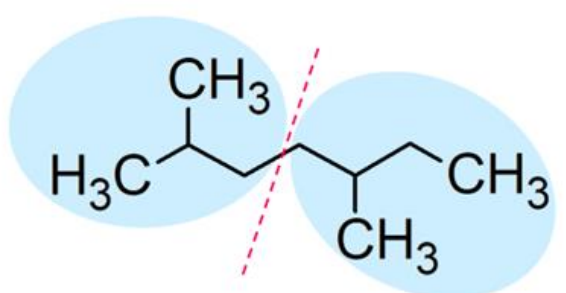

Figure 15: (a) Leucine with side chain mapping scheme and (b) 2,5-dimethylhexane. Dashed line shows that 2,5-dimethylhexane is a symmetric analogue of the leucine side chain.

The side chain of leucine had to be modeled as one bead because of the bond pattern. As with the isoleucine side chain, the most obvious analogue for leucine-isobutane-is a gas at room 
temperature and has the low condensation point of $-11.7^{\circ} \mathrm{C} .5^{2}$ So using the same approach as DeVane, et al.,23 we used 2,5-dimethylhexane--which is liquid at room temperature ${ }^{52--f o r}$ optimizing the non-bonded parameters of the leucine side chain. Figure 15 shows that the symmetry of 2,5-dimethylhexane provides two identical beads that are exact analogues of the leucine side chain.

\subsubsection{Lysine}

(a)

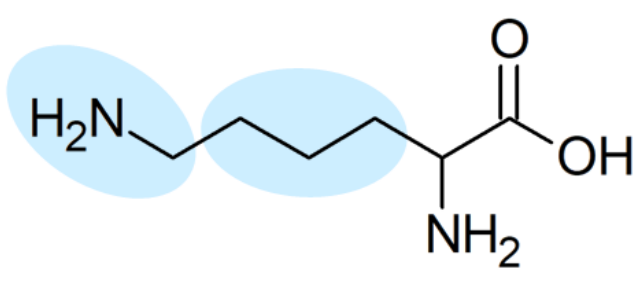

(b)

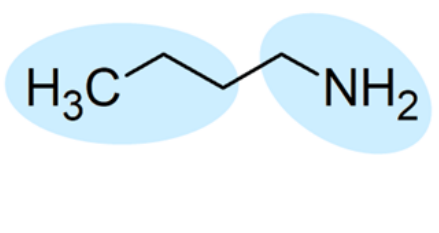

Figure 16: (a) Lysine with side chain mapping scheme and (b) N-butylamine

To model the neutral lysine side chain, we used N-butylamine to obtain non-bonded parameters for the outer side chain bead along with the C3M non-bonded parameters for the inner side chain bead. As shown in Figure 16, N-butylamine is a near-exact match to the lysine side chain.

\subsubsection{Methionine}

(a)

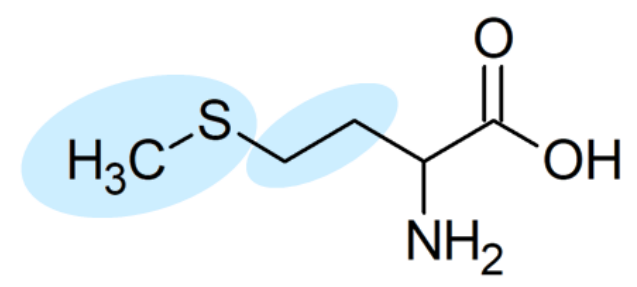

(b)

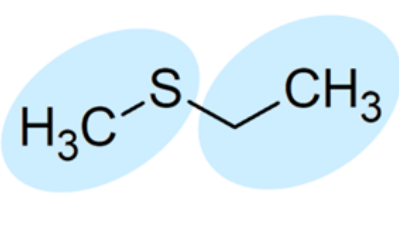

Figure 17: (a) Methionine with side chain mapping scheme and (b) ethyl methyl sulfide Ethyl methyl sulfide is the analogue of the methionine side chain, as can be seen in Figure 17. The C2M parameters were used for the inner side chain bead, leaving only the outer side chain bead-representing the sulfur atom and outermost carbon with associated hydrogens-to be optimized with respect to the experimental values of ethyl methyl sulfide. 


\subsubsection{Phenylalanine}

(a)

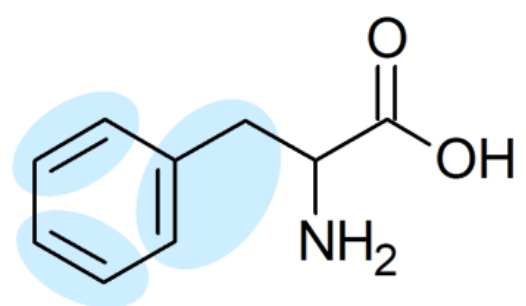

(b)

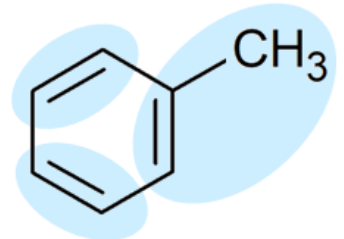

Figure 18: (a) Phenylalanine with side chain mapping scheme and (b) toluene

Toluene is the obvious analogue for phenylalanine, as shown in Figure 18. As our lab had already obtained non-bonded parameters for a two-carbon bead from modeling benzene (type BZ), ${ }^{33}$ those non-bonded parameters were used for the two-carbon side chain beads. That left only the three-carbon bead non-bonded parameters to be optimized. As in such cases with the other amino acid side chains, we verified this decision by comparing the RDFs of the toluene beads from using the BZ parameters with those of the AA mapped CG toluene RDFs and the phenylalanine side chain RDFs.

\subsubsection{Proline}

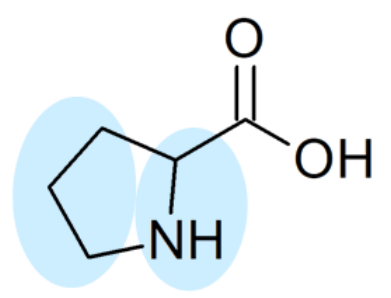

Figure 19: Proline with mapping scheme

Proline has the peculiar feature of having a side chain that is connected back to the amino acid nitrogen, as shown in Figure 19 above. It was decided that the three-carbon side chain bead could be modeled as C3M for its non-bonded parameters and that the nitrogen- $\mathrm{C}_{\alpha}$ bead could be modeled using the same parameters as those developed for the lysine outer side chain bead. Comparing RDFs of the CG proline beads and the RDFs of the AA mapped proline beads shows that this is not an unreasonable approximation. Pyrrolidine could be used to check or tune these 
parameters to potentially better fit the structure, but that did not seem likely to be necessary to capture the essential attributes of the proline beads. The tendency of proline to cause turns or kinks in a peptide or protein sequence is a more defining attribute of the amino acid, and that is more likely captured through the bond and angle distributions. The Val-Pro-Leu tripeptide was used to obtain those distributions.

\subsubsection{Serine}

(a)

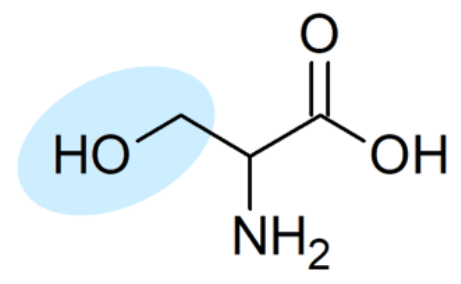

(b)

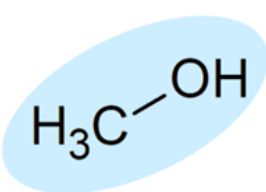

Figure 20: (a) Serine with side chain mapping scheme and (b) methanol

As can be seen in Figure 20 above, the obvious analogue for serine is methanol. Since methanol is a liquid at room temperature and is well-characterized with respect to its thermodynamic properties, there was no need to consider any other analogue option for obtaining the non-bonded parameters for the serine side chain bead.

\subsubsection{Threonine}

(a)

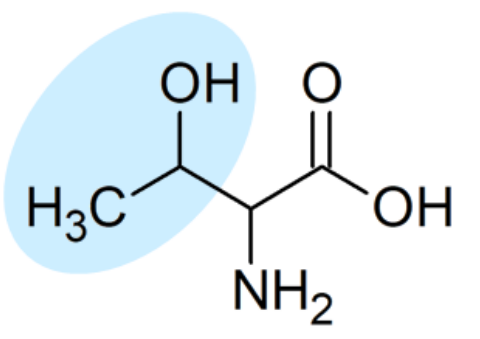

(b)

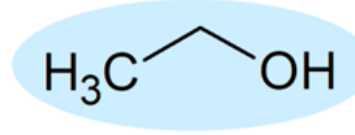

Figure 21: (a) Threonine with side chain mapping scheme and (b) ethanol

Ethanol is the analogue we used for the threonine side chain because of its similarity to the threonine side chain as shown in Figure 21. Like methanol for the serine side chain, ethanol is 
liquid at room temperature and well-characterized with respect to its thermodynamic properties such that no other analogue option was necessary.

\subsubsection{Tryptophan}

(a)

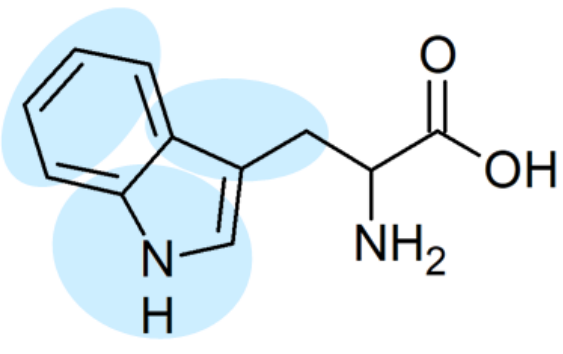

(b)

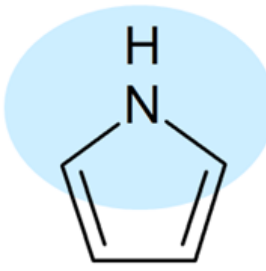

(c)

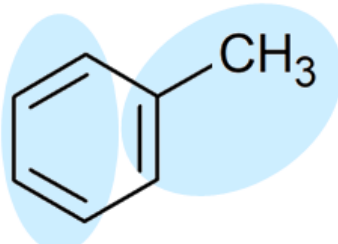

Figure 22: (a) Tryptophan with side chain mapping scheme, and analogues (a) pyrrole and (b) toluene

Tryptophan has the largest, bulkiest side chain. Initially we tried to use five beads and two rings for mapping the side chain, but we were not able to make that mapping scheme functional. When we attempted to run simulations with the five bead side chain, they were so unstable that they crashed immediately. This instability appeared to result from some beads in the side chain being counted in intramolecular interactions multiple times, which happened in part because of the two ring structures (resulting in multiple paths along which a bead could interact with others) and in part because only the interactions between beads directly bonded to each other were excluded. A four-bead ring was also ruled out as problematic, so the mapping scheme was instead finalized as one ring made up of three beads with the masses as similar as possible. As with histidine, however, the non-bonded parameters for these beads were optimized using more than one analoguetoluene and pyrrole in this case. The similarities of some of the constituents of these analogues to constituents in the tryptophan side chain are shown in Figure 22. 


\subsubsection{Tyrosine}

(a)

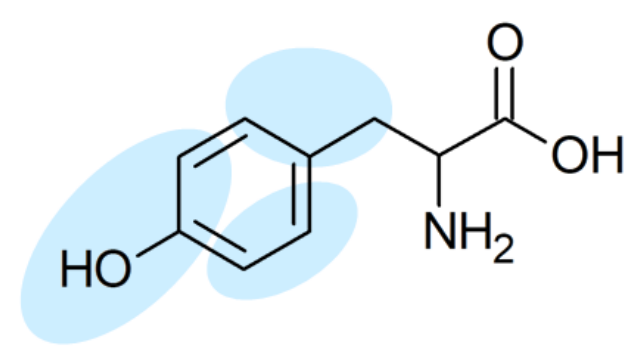

(b)

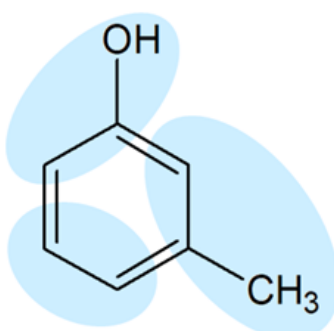

Figure 23: (a) Tyrosine with side chain mapping scheme and (b) m-cresol

The analogue chosen for tyrosine was m-cresol because of its similarities to the tyrosine side chain and because of the availability of experimental thermodynamic data at an appropriate temperature for parameterization. Because of the number of atoms in the tyrosine side chain and because of the way they are bonded to each other, a number of different options exist for the mapping scheme. The particular mapping scheme we settled on, as shown in Figure 23(a), preserves a ring structure for the CG tyrosine. Initially we attempted to model the tyrosine side chain with four beads, adhering to a preference for a 2:1 mapping scheme (two heavy atoms per bead). This mapping scheme proved unstable, however, so the final mapping scheme consists of only three beads. Toluene was used as the analogue for the three carbon bead, the two carbon bead was modeled with the same non-bonded parameters as those of the benzene bead, BZ, ${ }^{33}$ and the parameters for the bead with the hydroxyl group were optimized using m-cresol in combination with the already-developed non-bonded parameters for the other side chain beads. Because the structure of m-cresol differs slightly from that of the tyrosine side chain, however, the bonded parameters for tyrosine were set based on the AA CG mapped distribution of the tyrosine dipeptide rather than from the m-cresol analogue. 


\subsubsection{Valine}

(a)

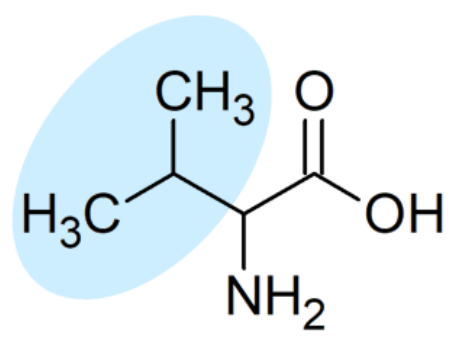

(b)

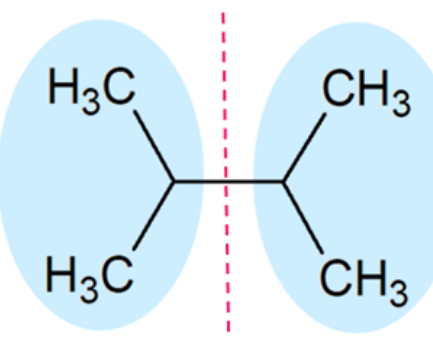

Figure 24: (a) Valine with side chain mapping scheme and (b) 2,3-dimethylbutane. Dashed line shows that 2,3-dimethylbutane is a symmetric analogue of the valine side chain.

The analogue used for valine was 2,3-dimethylbutane since it exists as a liquid at room temperature and since the more obvious analogue, propane, exists in the gas phase at that temperature. ${ }^{52}$ This is also the same procedure that DeVane, et al. used in the development of the SDK model. ${ }^{23}$ Figure 24 shows that the symmetry of 2,3-dimethylbutane provides two identical beads that are exact analogues of the valine side chain.

\subsubsection{Backbone and Termini}

(a)<smiles>[R]C(N)C(=O)NC([R2])C(=O)O</smiles>

(b)<smiles>CNC(C)=O</smiles>

(c)<smiles>CCC(=O)CC</smiles>

Figure 25: (a) A generic dipeptide showing the backbone mapping scheme, and analogues (b) N-methylacetamide and (c) diethylketone. 
(a)

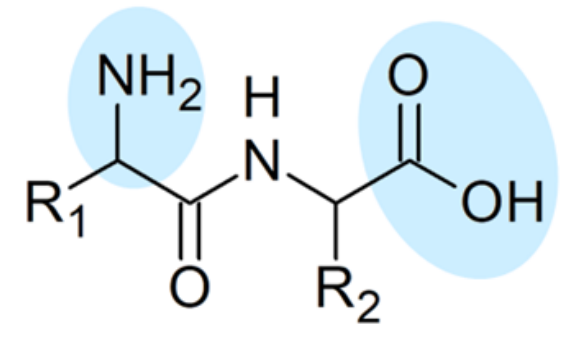

(b)

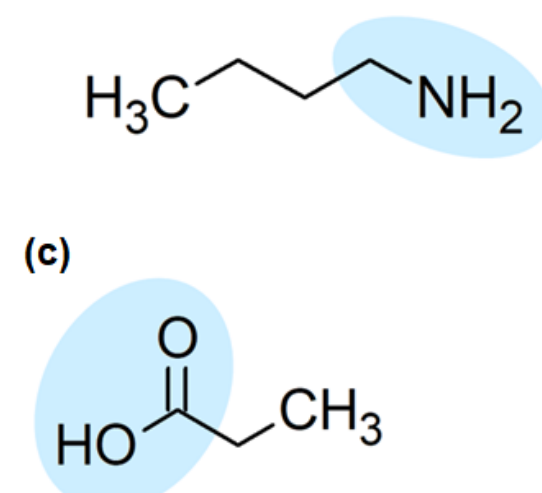

Figure 26: (a) A generic dipeptide showing the mapping scheme for the $\mathrm{N}$-terminus and the $\mathrm{C}$-terminus, and analogues (a) N-butylamine and (c) propionic acid.

In this model, there are two beads for modeling the standard peptide backbone and two beads for modeling the termini, as shown in Figures 25 and 26. This is different from most protein models which tend to use either one bead for the entire backbone repeat unit (Nitrogen, alphaCarbon, and carbonyl together) or which describe the backbone in AA detail, as shown in Table 1. Non-bonded parameters had already been determined by another group member for a bead with the same structure as the C-terminal bead when modeling propionic acid (unpublished results). The $\mathrm{N}$-terminal bead was given the same parameters as the outer lysine side chain bead. $\mathrm{N}$ methylacetamide was chosen as the analogue for the $\mathrm{N}-\mathrm{C}_{\alpha}$ bead (BNC), and diethylketone was chosen as the analogue for the backbone carbonyl (BCO). Simulations for N-methylacetamide were run at $313 \mathrm{~K}$ since its melting point is $28^{\circ} \mathrm{C}(301 \mathrm{~K}) .52$

\subsection{Bonded Parameters}

After the mapping schemes were determined, the next step that was undertaken was the running of AA simulations to obtain bond, angle, and distributions for the CG models by mapping the AA molecule to its CG version using the AA simulation trajectories. Considering that we needed to obtain parameters for bonds, angles, and dihedral angles related to the side chain beads, terminal beads, and peptide backbone beads, we decided to run AA simulations of predominantly 
homogeneous dipeptides-dipeptides that contain two of the same amino acid, e.g. alanyl-alanine and leucyl-leucine. This would result not only in bonds and angles unique to a given amino acid in a peptide, but it would also produce backbone bonds and angles and their force constants for each dipeptide simulation. Considering the uniqueness of the side chains of the different amino acids, the backbone bonds, angles, and force constants resulting from the AA simulations were likely to differ somewhat across dipeptide simulations. Thus we determined to use all of the dipeptide simulations in addition to a valine tripeptide simulation to define what would seem to be optimal bonded parameters for the backbone beads. Valine was chosen as the homogeneous tripeptide amino acid due to the moderate bulkiness of the side chain and single bead modeling of the side chain for ease of use. SDF (structure) files of the dipeptides were obtained from PubChem ${ }^{53}$ and converted to PDB files using the molecular editing software Avogadro. ${ }^{54}$ Because of the uniqueness of proline and arginine, non-homogeneous dipeptides were used to model those amino acids. Additionally, because we chose to bundle the side chain of alanine in with its terminal and backbone beads, we used a homogeneous tripeptide, alanyl-alanyl-alanine, in order to capture those angles and dihedrals. We also used structures that contained the L forms of the amino acids. For each dipeptide or tripeptide, Packmol ${ }^{55}$ was used to generate a PDB file containing five hundred dipeptide/tripeptide molecules. The box sizes varied because the sizes of the different dipeptides/tripeptides varied. Molecular dynamics simulations were run with the CHARMM18 force field and the molecular dynamics software NAMD ${ }^{56}$ (version 2.12), with the molecular visualization program VMD. ${ }^{57}$ The simulations were conducted at $298 \mathrm{~K}$ with a $1 \mathrm{fs}$ time step for $5 \mathrm{~ns}$, in the NPT ensemble (constant moles, pressure, and temperature) using Langevin dynamics for temperature control and the Nosé-Hoover Langevin piston barostat for pressure control. A configuration file is given in the appendix, A26, as an example. The Langevin thermostat is a popular choice for MD simulations. It produces the correct canonical distribution and controls temperature by removing energy from the system with friction forces and by adding energy to the system with random 
forces. ${ }^{58}$ The frictional and random forces are incorporated by adding them to the Newtonian equations of motion ${ }^{56}$ as shown in equation 6 in which $\gamma$ is the friction coefficient, $v$ is the velocity, $k_{B}$ is the Boltzmann constant, $T$ is temperature, $m$ is mass, and $R(t)$ is the univariate Gaussian random process. The fluctuating (random) force is described by the last term in equation 6 while the dissipative (frictional) force is described by the $-\gamma v$ term. Pressure control with the NoséHoover Langevin piston barostat used in NAMD generates the correct NPT ensemble distribution when used in conjunction with the Langevin thermostat. 56

$$
m a=F(r)-\gamma v+\sqrt{\frac{2 \gamma k_{B} T}{m}} R(t)
$$

NAMD implements the velocity-Verlet integration method for advancing the positions and velocities of each atom/bead in time, as shown in equations 7-10. The velocity-Verlet algorithm is simple, it is symplectic (demonstrating long-time stability) and time-reversible, and it requires only one force evaluation for each time step, making it suitable for biomolecular simulations which are typically large and complex. ${ }^{56}$

$$
\begin{gathered}
r(t+\Delta t)=r(t)+v(t) \Delta t+\frac{1}{2} a(t) \Delta t^{2} \\
v\left(t+\frac{\Delta t}{2}\right)=v(t)+\frac{1}{2}(t) \Delta t \\
a(t+\Delta t)=-\left(\frac{1}{m}\right) \nabla V[r(t+\Delta t)] \\
v(t+\Delta t)=v\left(t+\frac{\Delta t}{2}\right)+\frac{1}{2} a(t+\Delta t) \Delta t
\end{gathered}
$$

Particle Mesh Ewald (PME) was used for electrostatic interactions. PME is a method that divides the electrostatic interactions into a long-range contribution, which is calculated using a Fourier transform; and a short-range contribution, which is calculated in real space. PME is both highly accurate and reasonably fast, thus making it a standard choice for MD simulations. ${ }^{59}$ For the non-bonded interactions, a cutoff of $12 \AA$ was used with 1-4 scaling. With respect to the cutoff, this 
means that in the Lennard-Jones potential, calculations were performed only on atoms within $12 \AA$ from each other rather than allowing the potential to very gradually drift towards zero at long ranges. The 1-4 scaling means that within a molecule, non-bonded interactions between atoms located within two bonds from each other were ignored while the interactions between an atom and another atom three bonds away from it were calculated with reduced effect. Interactions between atoms farther apart from each other than three bonds in the same molecule would have full effect.

Following these AA simulations, the CG trajectory was created based on the AA simulation trajectory. The way this works is by identifying which atoms in a molecule make up which bead and then by obtaining the center of mass of those atoms which becomes the center of mass of the bead. Using these centers of mass, one can then calculate the length of the vector between two beads bonded to each other (i.e., the length of the bond between two beads bonded to each other), the angle created by three beads bonded to each other, and the dihedral angle created by four beads bonded to one another in series. All the lengths of a bond of a given type, all the angles of a given type, and all the dihedrals of a given type are collected and binned into histogram distributions. The resulting curves are used to obtain the equilibrium bond lengths and angles and approximations of the dihedral angles. These files also create a CG trajectory from the AA trajectory and from the information given about the mapping of the AA molecule to a CG molecule. This CG trajectory for each dipeptide/tripeptide simulation was loaded into VMD to obtain the radial distribution function (RDF) for each bead type with itself. The same procedure was carried out for the side chain and backbone analogues to check for consistency or discrepancy between the amino acid beads and the analogues used for obtaining optimal non-bonded parameters.

\subsection{Non-bonded Parameters}

The positions of the RDF peaks were used as center values for a narrow range given for optimization of the $r_{\min }$ values which are related to the $\sigma$ values of the LJ potential for the beads 
according to equation 11 . The RDF is a measure of the probability of finding another particle (e.g. atom, bead) within any given distance from the particle itself. For any substance, the likelihood of finding a particle of its own kind within a distance less than that of the diameter of the particle is essentially zero because of the strong repulsive force that prohibits the overlapping of particles. But for a closely packed structure like a liquid, there will be a high probability of finding another particle at a distance approximately equal to the diameter of the particle (Chandler, 1987).60 This high probability corresponds to the first peak of a graph of the RDF. In this way, the RDF can be used to obtain the $\sigma$ values of the LJ potential since $\sigma$ represents the distance at which the potential between two particles is zero. Ranges for the $\varepsilon$ values of the LJ potential were given a little wider and were based on the polarities of the atoms represented by the beads; for example, a maximum lower bound was typically -1.0 while a maximum upper bound was usually around -0.3 . Hydrocarbon beads tend to have less negative epsilon values while beads containing one or more nitrogen or oxygen atoms tends to have more negative epsilon values. The optimization algorithm can then select values from those ranges for testing in simulations, and the parameters were optimized by comparing the heat of vaporization, surface tension, and density values of the CG analogue simulations with the experimental values for those analogues. Particle Swarm Optimization (PSO) was used for this purpose.

$$
r_{\min }=2^{1 / 6 \sigma}
$$

PSO is an algorithm that takes inspiration from the movements of a flock of birds or swarm of insects. To begin with, a set of values (a "particle") for the force-field parameters to be optimized is randomly selected based on the supplied ranges for use in a test simulation. Many such particles are generated for simultaneous test simulations. Each particle is analogous to a bird in a flock (or insect in a swarm). In our case, we chose to generate forty such particles for each iteration of the PSO for broad coverage of the parameter space to facilitate quicker identification of optimal force field parameters. Once the heat of vaporization, surface tension, and density were calculated for 
each simulation, they were compared with the experimental values and ranked based on the errors. The best set is that which corresponds to the lowest error. For the subsequent iteration, the best particle remains the same, and all other particles have their values adjusted slightly in the direction of the values of the best particle. A record is also kept of an individual particle's best set-and updated when it changes - so that the particle may be moved in that direction as well. The maximum amount by which each value can change is given by the investigator. PSO is implemented using equations (12) and (13) in which $V$ represents how quickly a given value changes (its velocity), $x$ represents the value itself, the subscript $n$ stands for the current step, and $n+1$ stands for the next step. The global best (the best set of values across all iterations) is represented by $\mathrm{g}_{\text {best, }}$ and $\mathrm{p}_{\text {best }}$ is for the personal best set of a particle. The value of rand $($ ) is a random number between 0 and $1, w$ is an inertia factor, and $c 1$ and $c 2$ are swarm and personal constants, respectively. The first term in equation (12) is an inertial term that stabilizes the particle motion, and the constants $c 1$ and c2 give the relative magnitude or pull in the direction of the global best and personal best, respectively. The position of a particle is updated based on the distance traveled over a discrete time interval $(\Delta t=1) \cdot{ }^{33}$ The investigator can set the number of iterations to be completed. In the case of this study, PSO was able to minimize the overall error within the first 40 or so iterations when using 40 particles.

$$
\begin{gathered}
V_{n+1}=w * V_{n}+c 1 * \operatorname{rand}() *\left(g_{\text {best }, n}-x_{n}\right)+c 2 * \operatorname{rand}() *\left(p_{\text {best }, n}-x_{n}\right) \\
x_{n+1}=x_{n}+V_{n+1} * \Delta t
\end{gathered}
$$

In addition to the PSO, an artificial neural network (ANN) was incorporated after the first four iterations. The data from those iterations - and all subsequent iterations-were given to the ANN which used the data to produce a parameter set for each following iteration that seemed likely—based on the ANN computations—-to result in a reduced overall error. The ANN works in the following manner. A set of input values comprise the first "layer." The final layer is comprised of the output values - target values or predictions. Any layers between the input and output layers 
are called hidden layers, and they are comprised of "nodes" which are essentially processing centers, playing a role like that of biological neurons. Layers are connected to each other, receiving inputs from the nodes of the previous layer, and sending outputs to the next layer, except in the cases of the input layer-which has no inputs to itself-and the output layer, which does not send output on to any further layers. The inputs to a node are individually weighted (with weights that can be tuned), and they are summed together and passed through an activation function to produce the output of the node. The activation function acts like an on/off switch such that the output is only passed on to the next layer if a certain threshold value is reached. ${ }^{33}$ For this research study, TensorFlow 61 (a Python library created by Google developers) was utilized for implementation of the ANN with the exponential linear unit (ELU) activation function; an input layer, five hidden layers, and an output layer; and dropout to help prevent overfitting. The number of input nodes corresponded to the number of target properties for each system (e.g. targeting density, heat of vaporization, and surface tension corresponds to three input nodes), and the number of nodes in the output layer corresponded to the number of force field parameters to be fitted. Each hidden layer contained fifty nodes. Incorporating the ANN into the PSO is not absolutely necessary for achieving good results, but it can lead to a satisfactorily minimal error sooner than PSO alone and is not in any way detrimental. ${ }^{33}$ In some cases, it was even able to lead to more accurate predictions if the given ranges for the PSO did not encompass the best values, thus guiding the model development. Figure 27 illustrates how the ANN is incorporated into the optimization algorithm. 


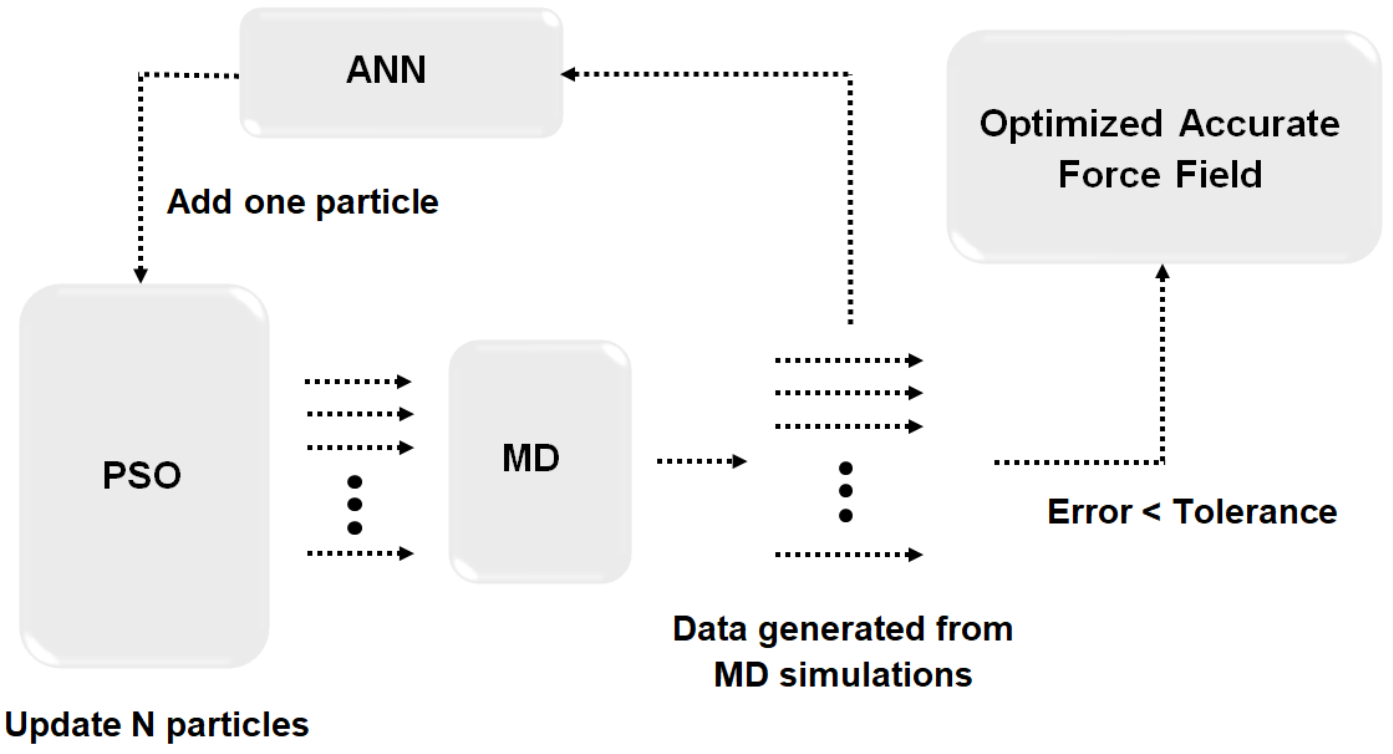

Figure 27: Schematic showing PSO and ANN incorporation with MD simulations to develop optimized force field parameters.

\subsection{Calculation of Thermodynamic Properties}

To obtain the density of a simulation, the number of molecules, the molecular weight of each molecule, and the simulation box size must be known. The density was calculated according to equation (14) below in which $\rho, m$, and $V$ have their usual meanings of density, mass, and volume, respectively. $N$ stands for the number of molecules in the system, $M W$ is the molecular weight, $N_{A}$ is Avogadro's number, and $L_{x}, L_{y}$, and $L_{z}$ are the box lengths in the $\mathrm{x}, \mathrm{y}$, and z dimensions, respectively.

$$
\rho=\frac{m}{V}=\frac{\left(\frac{N \times M W}{N_{A}}\right)}{L_{x} L_{y} L_{z}}
$$

The surface tension was calculated using equation 15 in which $L_{z}$ is the box length in the $\mathrm{z}$ dimension, and $P_{z z}, P_{x x}$, and $P_{y y}$ are the pressure tensors in the $\mathrm{z}, \mathrm{x}$, and y dimensions, respectively. The simulation is run at constant volume with the box length extended in the $\mathrm{z}$ dimension to the extent that a region of vacuum is created above the liquid surface.

$$
\gamma=\frac{L_{z}}{2}\left[P_{z z}-\frac{\left(P_{x x}+P_{y y}\right)}{2}\right]
$$


The heat of vaporization $\left(\Delta H_{\text {vap }}\right)$ was calculated using equation (19). The volume of the liquid phase, $V_{l i q}$, is negligible as compared with the volume of the gas phase, $V_{\text {gas }}$; and with the assumption that the gas is an ideal gas such that the kinetic energies of the molecule in the gas and liquid phases are the same, equation (17) becomes equation (18). $C$ is a correction term that is usually small and neglected, giving equation (19) which is used for calculating the heat of vaporization from MD simulations in which $T$ is the temperature of the simulation. ${ }^{62} R$ is the ideal gas constant. The potential energy of the gas phase, $E_{\text {gas }}^{\text {potential }}$, is obtained by simulating one molecule in a box at constant volume to imitate the lack of intermolecular interactions experienced by a molecule in the gas phase as per the ideal gas law. The potential energy of the liquid phase, $E_{\text {liq }}^{\text {potential }}$, is obtained from the same simulation conducted to determine the density. Since the potential energy is a combination of the intermolecular and intramolecular energies, the potential energy of the gas phase can be set as zero for CG simulations in which a molecule is represented as a single bead.

$$
\begin{gathered}
\Delta H_{\text {vap }}=H_{\text {vapor }}-H_{\text {liquid }} \\
\Delta H_{\text {vap }}=E_{\text {gas }}(T)-E_{\text {liq }}(T)+p\left(V_{\text {gas }}-V_{\text {liq }}\right) \\
\Delta H_{\text {vap }}=E_{\text {gas }}^{\text {potential }}(T)-E_{\text {liq }}^{\text {potential }}(T)+R T+C \\
\Delta H_{\text {vap }}=E_{\text {gas }}^{\text {potential }}(T)-E_{\text {liq }}^{\text {potential }}(T)+R T
\end{gathered}
$$

Five hundred molecules were used for the density and surface tension simulations. Each simulation-for density, surface tension, and heat of vaporization-was run for a total of 2 ns. Nonbonded interactions were calculated by excluding 1-2 interactions and with a cutoff of $12 \AA$. Constant temperature was simulated using the Langevin thermostat, and constant pressure simulations were conducted with the Nosé-Hoover Langevin piston barostat 56 as described in the previous section. 


\subsection{Results and Discussion}

Prior to moving forward with setting the non-bonded parameters for the backbone and termini beads as the same across all dipeptides, a quick comparison of the AA CG mapped RDFs of the backbone and termini beads showed similarities as can be seen in Figure 28. This provided verification that it would be suitable to treat the beads as the same regardless of which amino acid they are associated with. The RDFs also indicate that the environment of a bead-which other beads are attached to the molecule and are in the vicinity, as well as their sizes and crowding effects-can influence the RDF.

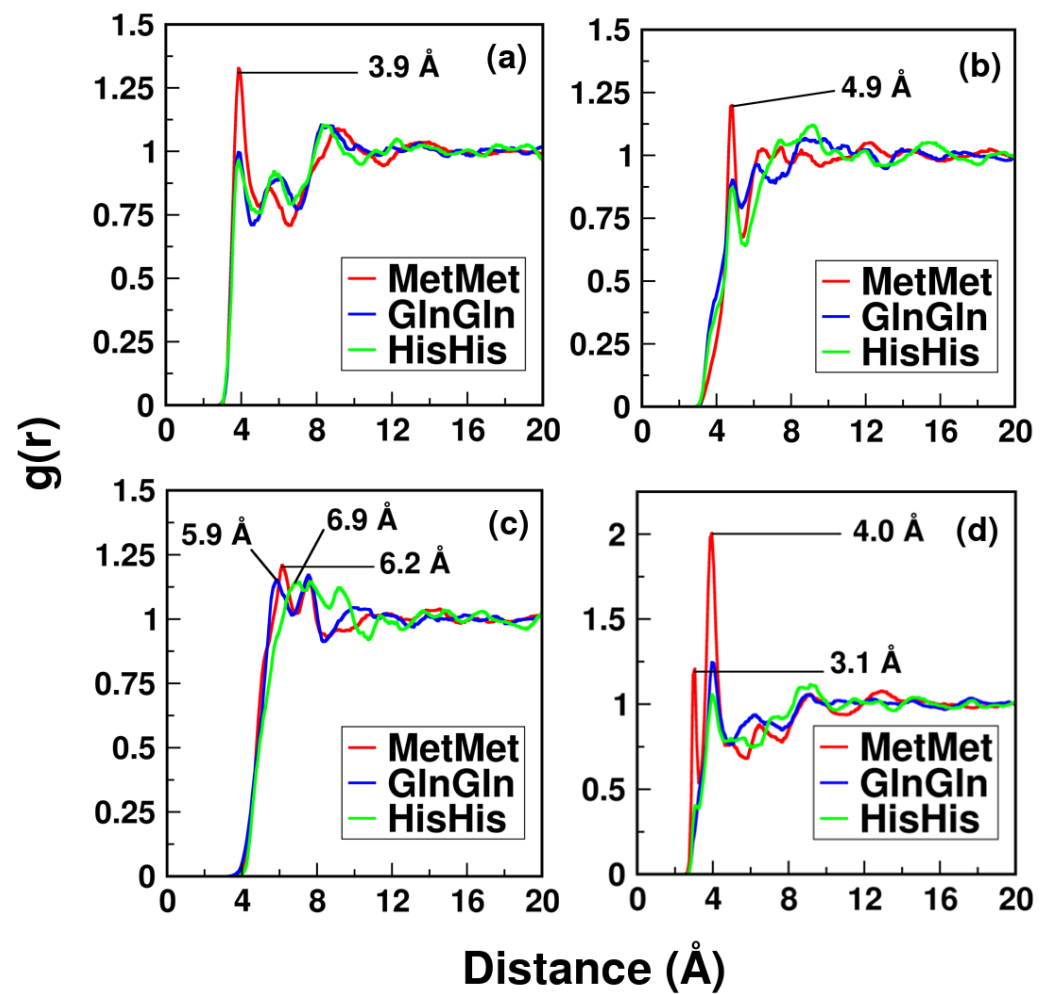

Figure 28: AA RDFs of (a) NCT, (b) CO, (c) NC, and (d) COO1 beads from the homogeneous methionine (MetMet), glutamine (GlnGln), and histidine (HisHis) dipeptides.

After the desired number of optimization cycles (100 at least with a maximum of 200, depending on the dipeptide) the top typically 1-3 sets with the lowest errors were selected for testing over ten nanoseconds. This allowed the selection of optimal non-bonded parameters for each bead type. It was also an important verification step because the top PSO result did not necessarily correlate with the top result after 10 ns simulations. While the density error remained 
essentially the same for the 2 ns optimization cycle and the $10 \mathrm{~ns}$ simulation, the heat of vaporization and surface tension errors in some cases were different such that the error showed an increase up to about $6 \%$ (e.g. from $2 \%$ error to $8 \%$ error). Additionally, we targeted a very low density error-within $2 \%$ of the experimental value; a surface tension error within $5 \%$ of the experimental value; and a heat of vaporization error as low as possible while optimizing the density and surface tension errors. The heat of vaporization error tends to be much higher, perhaps partly because the energy landscape for a CG model is much smoother than that of an AA model and the energy landscape in reality. ${ }^{6}$

Interestingly, the analogues methanol (for serine), ethanol (for threonine), and m-cresol (for tyrosine) all showed the worst matches to their experimental heat of vaporization values by far, with the errors for the CG models being $52 \%$ for both methanol and ethanol and $29 \%$ for mcresol. Since these three analogues all contain a hydroxyl group--and since they are the only analogues that contain a hydroxyl group--it seems likely that the peculiar hydrogen bonding capacity of the hydroxyl group may be the cause of the extremely high heat of vaporization errors. The analogues containing an amine group do not exhibit such poor matches to the experimental heat of vaporization values despite their hydrogen bonding capacity, so in the cases of methanol, ethanol, and m-cresol, it appears to be the oxygen atom that makes the difference. However, the CG water models previously developed by Bejagam, et al. ${ }^{33}$ do not have such high errors with respect to the heat of vaporization values. So perhaps the results shown here have not only to do with the character of the hydroxyl group, but also the character of the molecules in that the carboncontaining segments are quite different in their properties from the hydroxyl group itself. Further investigation would be useful in helping to elucidate and overcome this issue.

With respect to the RDFs - which are impacted by the non-bonded parameters, not the bonded parameters-the CG bead peaks are usually much sharper and higher than those of the AA CG-mapped peaks. Frequently, if there is a shoulder or a more gradual slope in the AA RDF initial 
peak, the CG initial peak exists where the shoulder is, or in the middle of the slope, or between the shoulder and the initial peak when comparing with the AA CG-mapped distribution. The RDFs for the methionine terminal and backbone beads are shown in Figure 29, comparing the AA CG mapped distributions with the CG distributions. Methionine was chosen as the example for the backbone and terminal beads because its AA RDFs are the most pronounced, as shown in Figure 28. The results for the NCT (neutral N-terminal), CO (backbone carbonyl bead), and NC (backbone N-C $\alpha$ ) beads seem to indicate that the analogues chosen for the backbone and terminal beads were reasonable. The CG RDF for the CO01 (neutral C-terminal) bead (Figure 29(d)) is a rather poor match to the AA RDF. However, because the C001 bead represents a carboxyl functional group, and the carboxyl functional group of propionic acid was used as the analogue, there is no reason to suspect that propionic acid was not a sufficiently good analogue to represent the C-terminal bead, C001. Furthermore, the result shown is essentially the same when comparing the CG RDF of the propionic acid bead with the AA CG-mapped RDF of the propionic acid bead. This indicates that a simple coarse-graining of the carboxyl group fails to capture the AA details of the carboxyl group. Comparisons of the CG RDFs for each dipeptide side chain bead type with their AA mapped CG counterparts show that the analogue choices for those bead types were suitable as well; all such figures are given in the appendix for the reader's examination. Considering the unusual nature of the proline side chain, as discussed previously, it should be noted that the CG RDFs of the proline backbone (PNC) and side chain (PS) beads correspond well to their AA CG-mapped RDFs, as shown in Figure A18.6 in the appendix. 


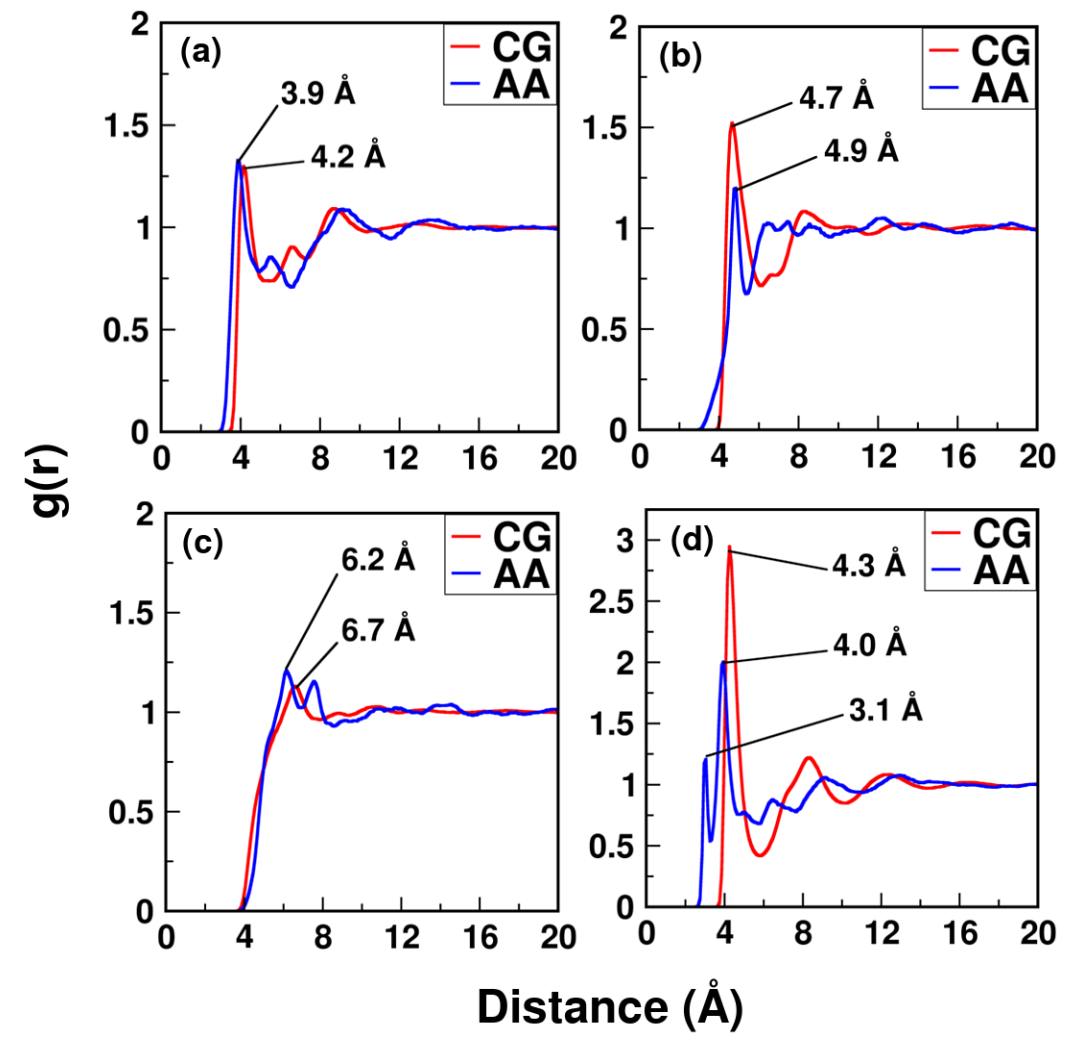

Figure 29: Comparisons of $A A$ coarse-grain mapped (AA) RDFs vs coarse-grained (CG) RDFs of (a) NCT, (b) CO, (c) NC, and (d) COO1 from the methionine dipeptide.

Following the 10 ns simulations to finalize the non-bonded parameters, 100 ns simulations were conducted to further validate these parameters. The trajectory from each simulation was divided into segments from 50-60 ns, 60-70 ns, 70-80 ns, 80-90 ns, and 90-100 ns to obtain block average values for the density, surface tension, and heat of vaporization; these values are reported in Tables A2.1-A2.3 in the appendix and shown in Figures 30-32 below. Percent errors were calculated in accordance with equation 20 .

$$
\% \text { error }=\frac{\mid \text { experimental }-C G \text { model } \mid}{\text { experimental }}
$$




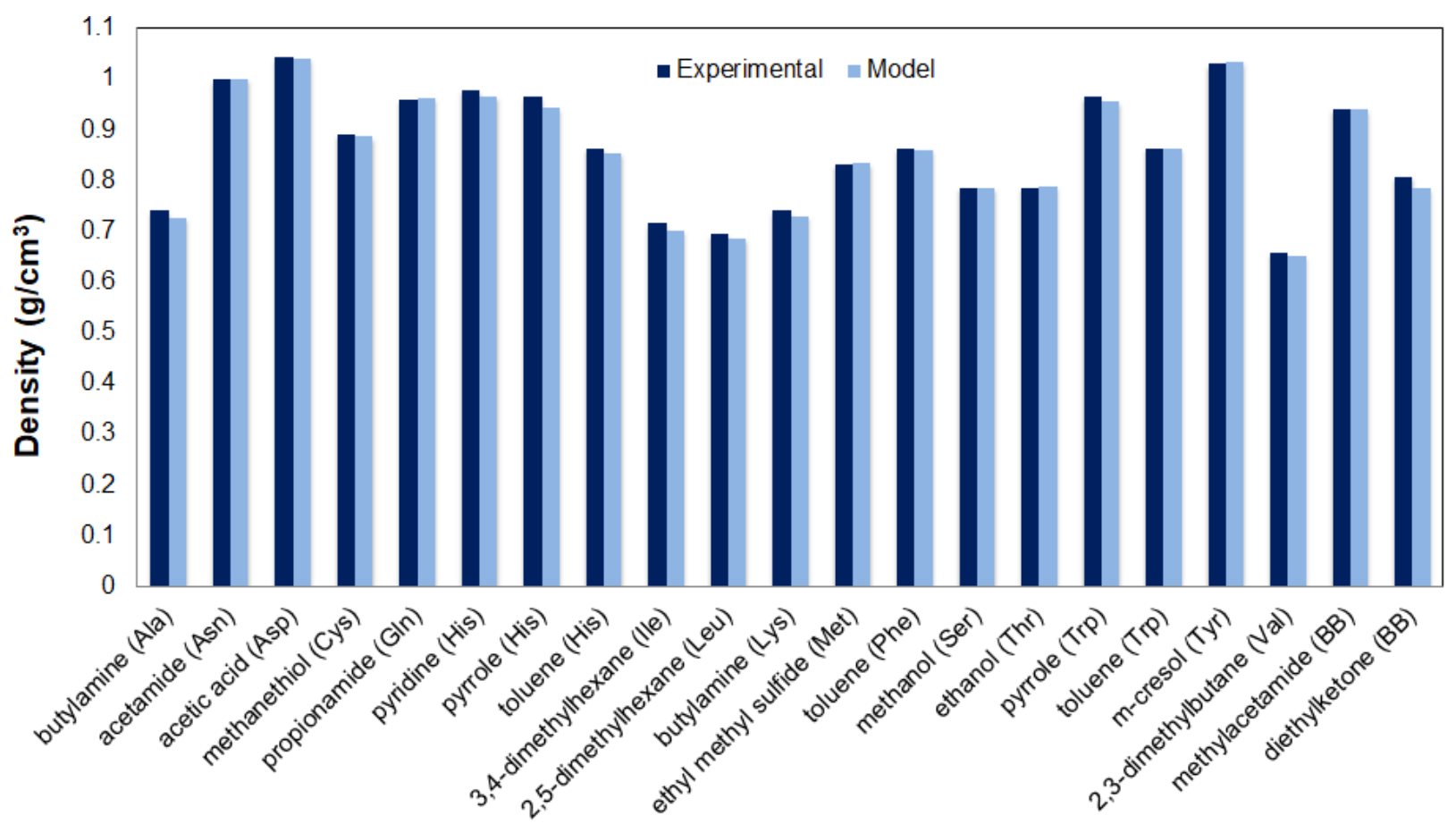

Analogue

Figure 30: Comparisons of experimental and CG model densities. BB stands for backbone. Experimental data from refs ${ }^{63-67}$.

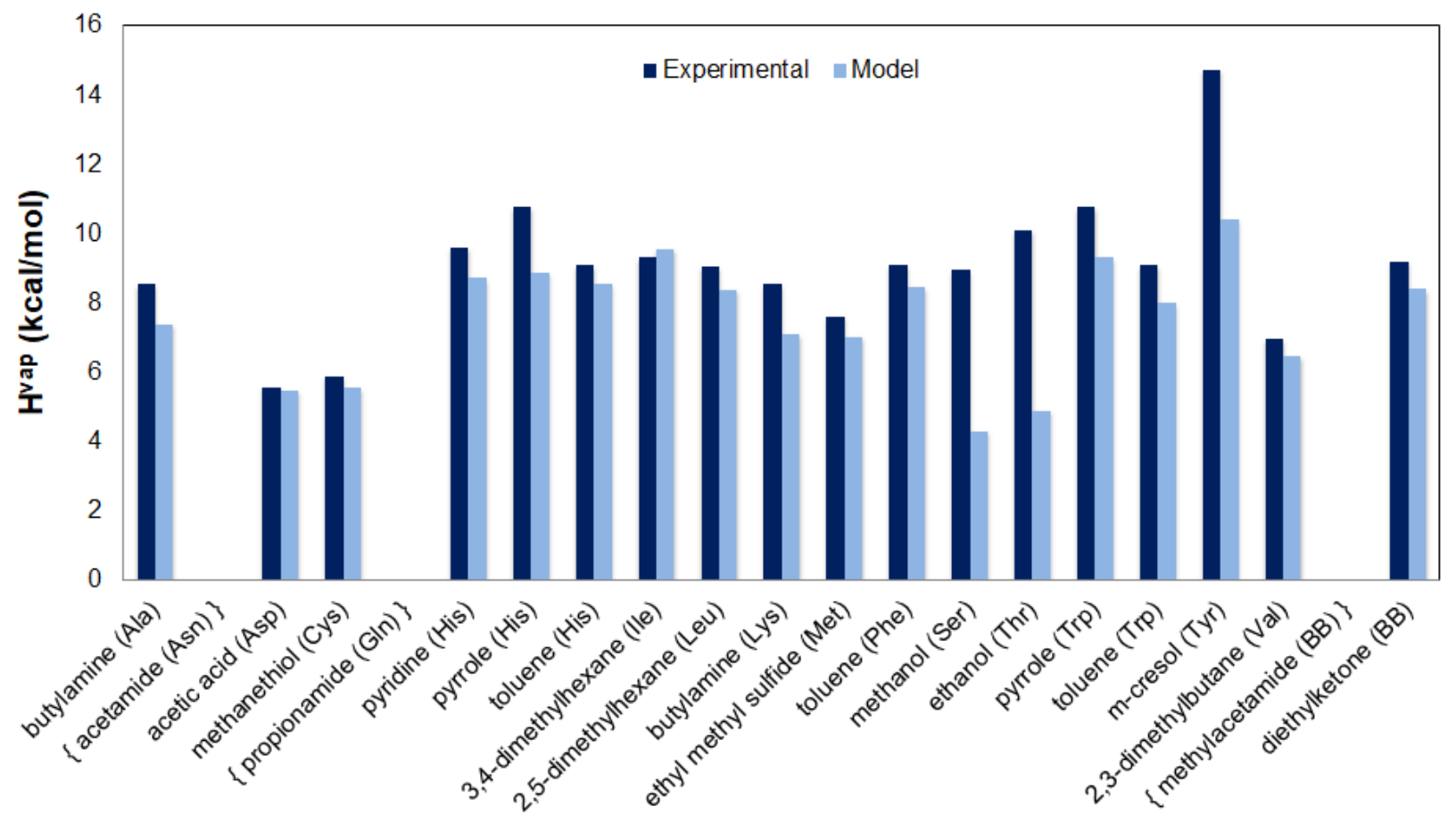

\section{Analogue}

Figure 31: Comparisons of experimental and CG model heats of vaporization. BB stands for backbone. Experimental data from refs ${ }^{63,65,68}$. Experimental values for heat of vaporization for molecules in braces $\{$ example $\}$ were not found. 


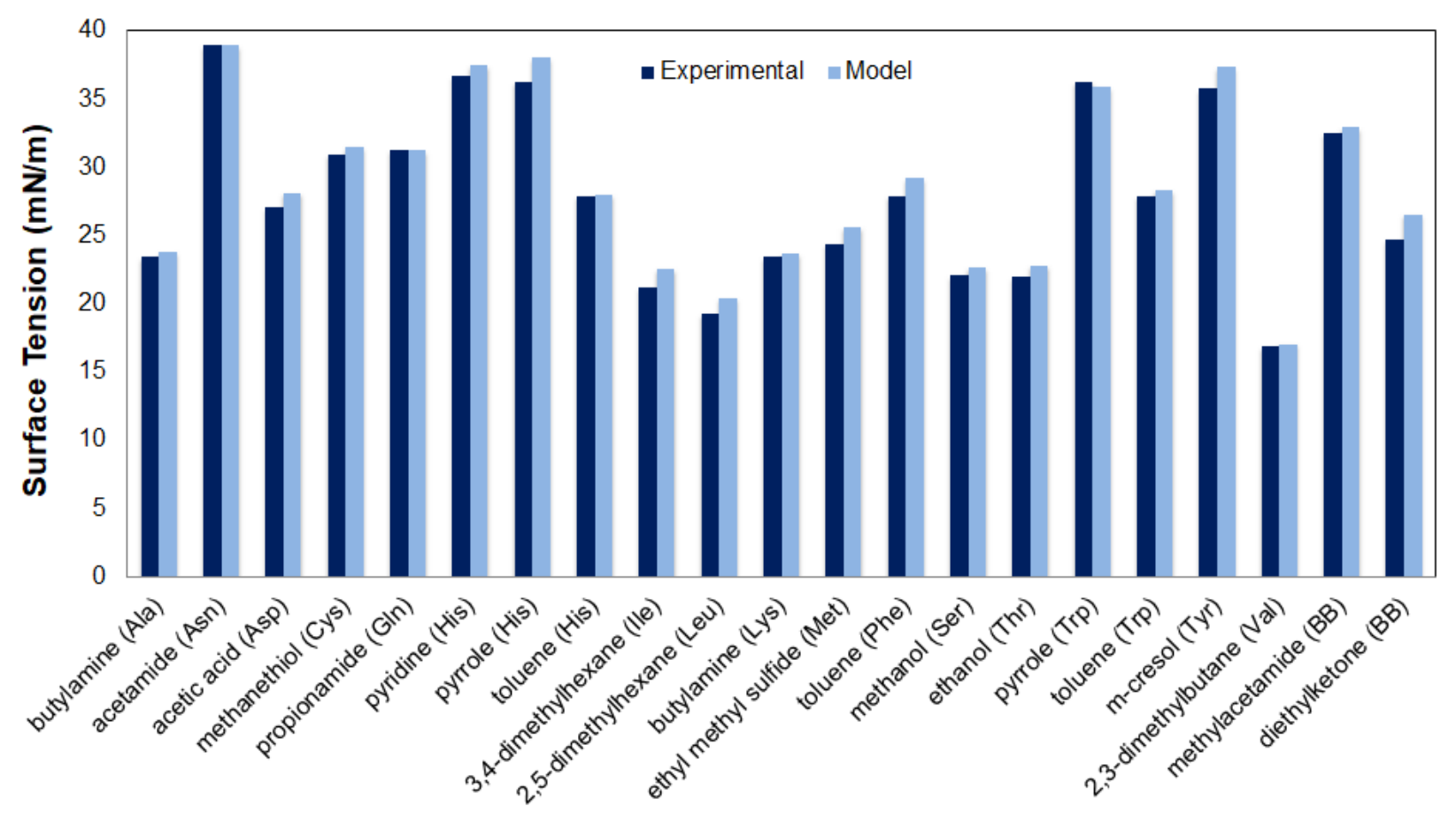

Analogue

Figure 32: Bar graph comparisons of experimental and CG model surface tensions. BB stands for backbone. Experimental data from refs ${ }^{63-66,68,69}$.

With the non-bonded parameters having been determined for the amino acid side chain and backbone beads, those values were plugged back into the dipeptide parameter files to finalize the bonded parameters and test the CG dipeptide simulation densities against the AA dipeptide simulation densities. The density results are given in Table 3 with percent errors calculated in accordance with equation 20 above with the substitution of the AA value in place of experimental. For the most part the density errors were not large, but in the case of the glycine dipeptide the error was $11.73 \%$. As will be shown in some paragraphs that follow, this error-and that for any of the dipeptides, by extension—can vary dramatically based on the non-bonded parameters used and the exclusion criteria provided in the configuration file for a simulation. The bond, angle, and dihedral distributions, along with mapping schemes and RDFs, are included in the appendix, for both analogues and peptides; the graphs represent the best outcomes from optimization and 
selection of the non-bonded and bonded parameters given some of the constraints described in the paragraphs below.

Table 3: Density comparisons, all atom (AA) vs coarse-grained (CG) models

\begin{tabular}{cccc}
\hline Dipeptide/Tripeptide & AA Density $\left(\mathbf{g} / \mathbf{c m}^{3}\right)$ & CG Density $\left(\mathrm{g} / \mathbf{c m}^{3}\right)$ & Percent Error \\
\hline Ala-Ala-Ala & 1.170 & 1.169 & 0.16 \\
His-His & 1.331 & 1.250 & 6.11 \\
Leu-Leu & 1.011 & 0.991 & 2.00 \\
Lys-Lys & 1.120 & 1.067 & 4.73 \\
Asn-Asn & 1.358 & 1.290 & 4.99 \\
Asp-Asp & 1.371 & 1.335 & 2.66 \\
Ala-Arg & 1.207 & 1.194 & 1.05 \\
Cys-Cys & 1.359 & 1.259 & 7.39 \\
Gln-Gln & 1.277 & 1.283 & 0.47 \\
Glu-Glu & 1.289 & 1.233 & 4.32 \\
Gly-Gly & 1.321 & 1.166 & 11.73 \\
Ile-Ile & 1.014 & 0.987 & 2.71 \\
Met-Met & 1.188 & 1.161 & 2.25 \\
Phe-Phe & 1.134 & 1.123 & 1.03 \\
Val-Pro-Leu & 1.062 & 1.021 & 3.91 \\
Ser-Ser & 1.337 & 1.245 & 6.87 \\
Thr-Thr & 1.179 & 1.193 & 1.24 \\
Trp-Trp & 1.218 & 1.244 & 2.11 \\
Tyr-Tyr & 1.204 & 1.238 & 2.88 \\
Val-Val & 1.039 & 0.977 & 5.98 \\
\hline average \% error &. &. & 3.73 \\
\hline
\end{tabular}

In this final parameterization for setting the bonded parameters, there were four primary matters of importance that had to be taken into consideration and addressed. The first was that CG distributions are much more uniform than AA distributions often are. While an AA distribution may have, for example, two peaks in a bond distribution, the CG distribution will have only one. 
This is also true of the angle distributions. The dihedral distributions may have more than one peak in the CG distribution, but in some cases many different attempts to find a matching dihedral for the CG model failed, and the only outcome that could be obtained was a dihedral that was shifted (as in figure A10.5(e)) or a non-existent (flat) dihedral (as in Figure A9.5(e)). Good matches could be obtained for many of the dihedrals, however, as shown in the dihedral figures in the appendix.

The second matter of note is that higher force constants require a smaller time step because a stiffer force constant engenders a higher vibrational frequency which correlates with smaller time steps. ${ }^{49}$ This meant that in cases such as the bonds and angles in ring structures (e.g. the side chain of Histidine), while the all-atom distribution might have required a very high force constant to match it, the final force constant for each bond and angle given as the CG parameter was lower to help ensure stability at a time step of 5 femtoseconds or greater. The lower the time step, the longer it takes for the simulation to reach microseconds in length; therefore it is preferable to have as high a time step as seems to be reasonable considering the parameterization. Figure 33 shows, as an example, the AA and CG distributions for a bond and an angle in the Histidine side chain.
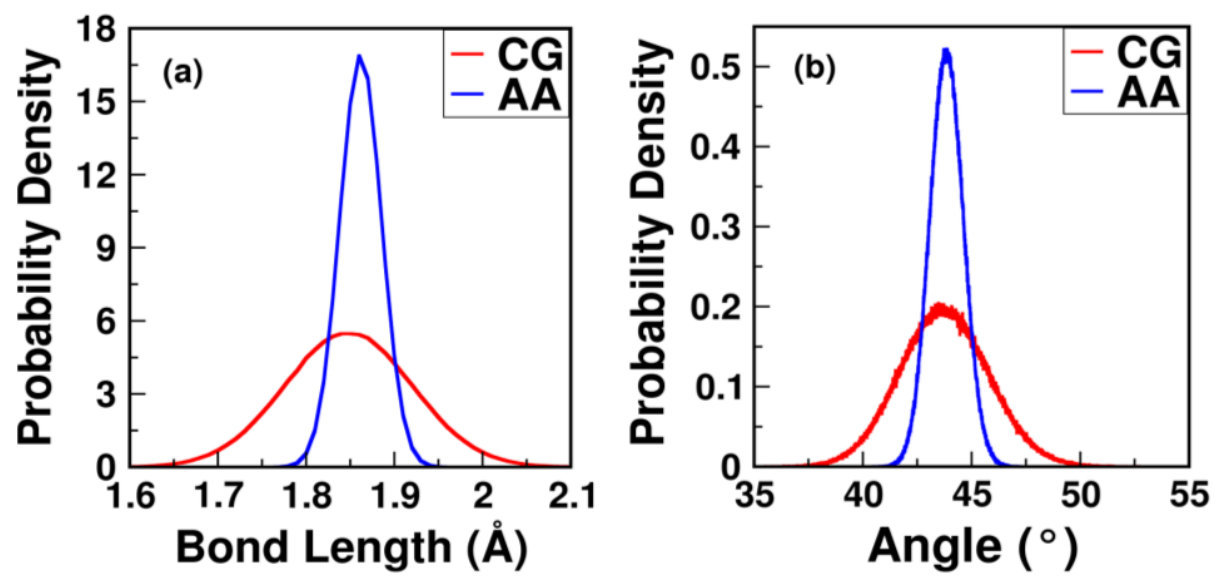

Figure 33: Comparisons of all-atom (AA) mapped and coarse-grained (CG) distributions in (a) bond NCR2-NCR1 and (b) angle NCR2-CCR1-NCR1 in the histidine side chain.

A third matter of importance was to parameterize the bonded interactions in such a way as to harmonize them across the backbones of the different dipeptides. As was mentioned previously, there are certain backbone bonds and angles such as CO-NC and CO-NC-COO1 that were in most of 
the dipeptide simulations, and we decided to consider all the dipeptide distributions and attempt to harmonize them to obtain the most reasonable parameters. As it turned out, this was not challenging to do since the common bonds and angles were largely consistent across dipeptides as can be seen in Figures 34, 35, and 36 below.
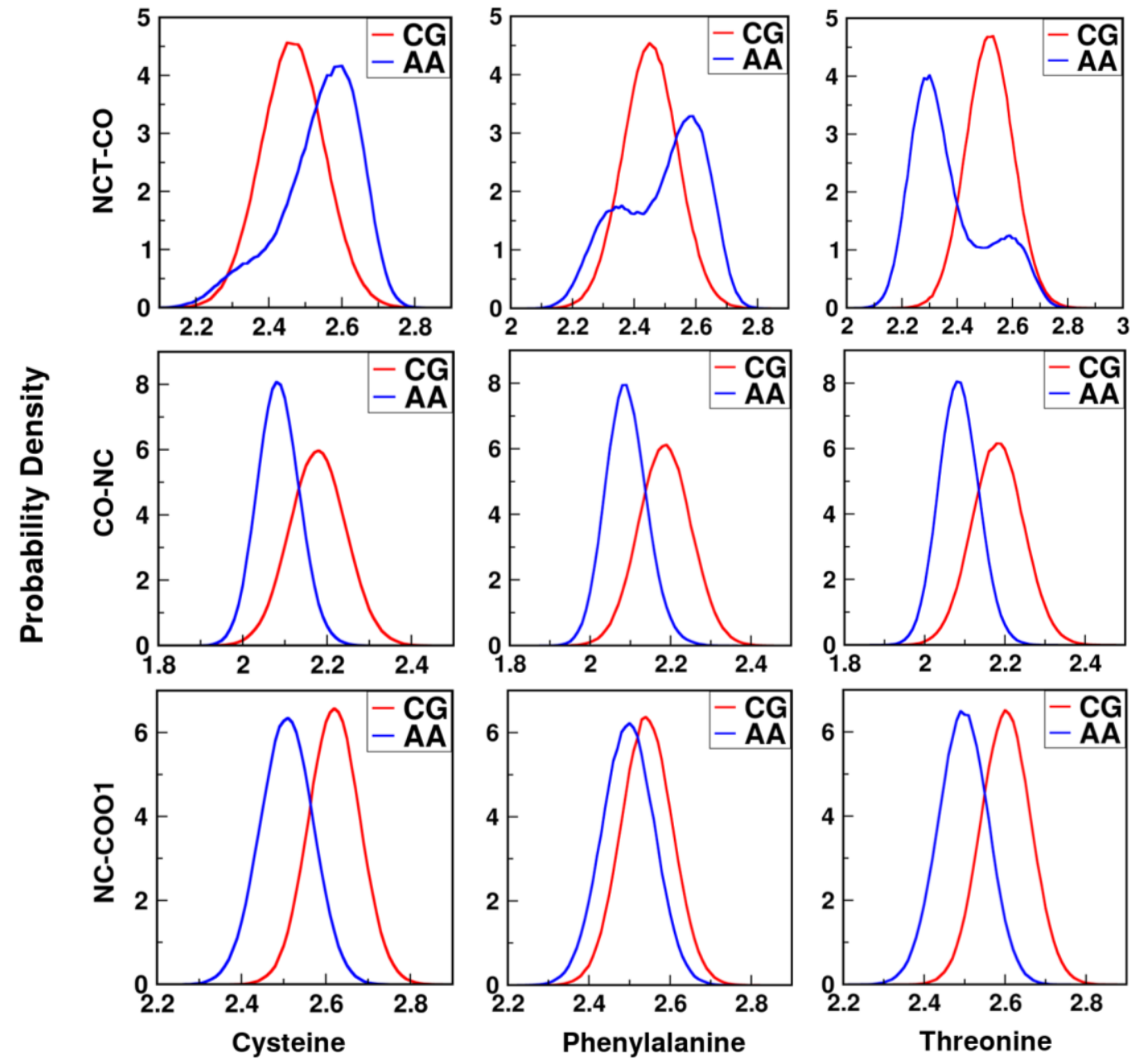

Bond Length ( $\AA$ )

Figure 34: Comparisons of backbone bond distributions for the bonds NCT-CO (top row), CO-NC (middle row), and NC-COO1 (bottom row) from the cysteine (first column), phenylalanine (second column), and threonine (third column) dipeptides. 

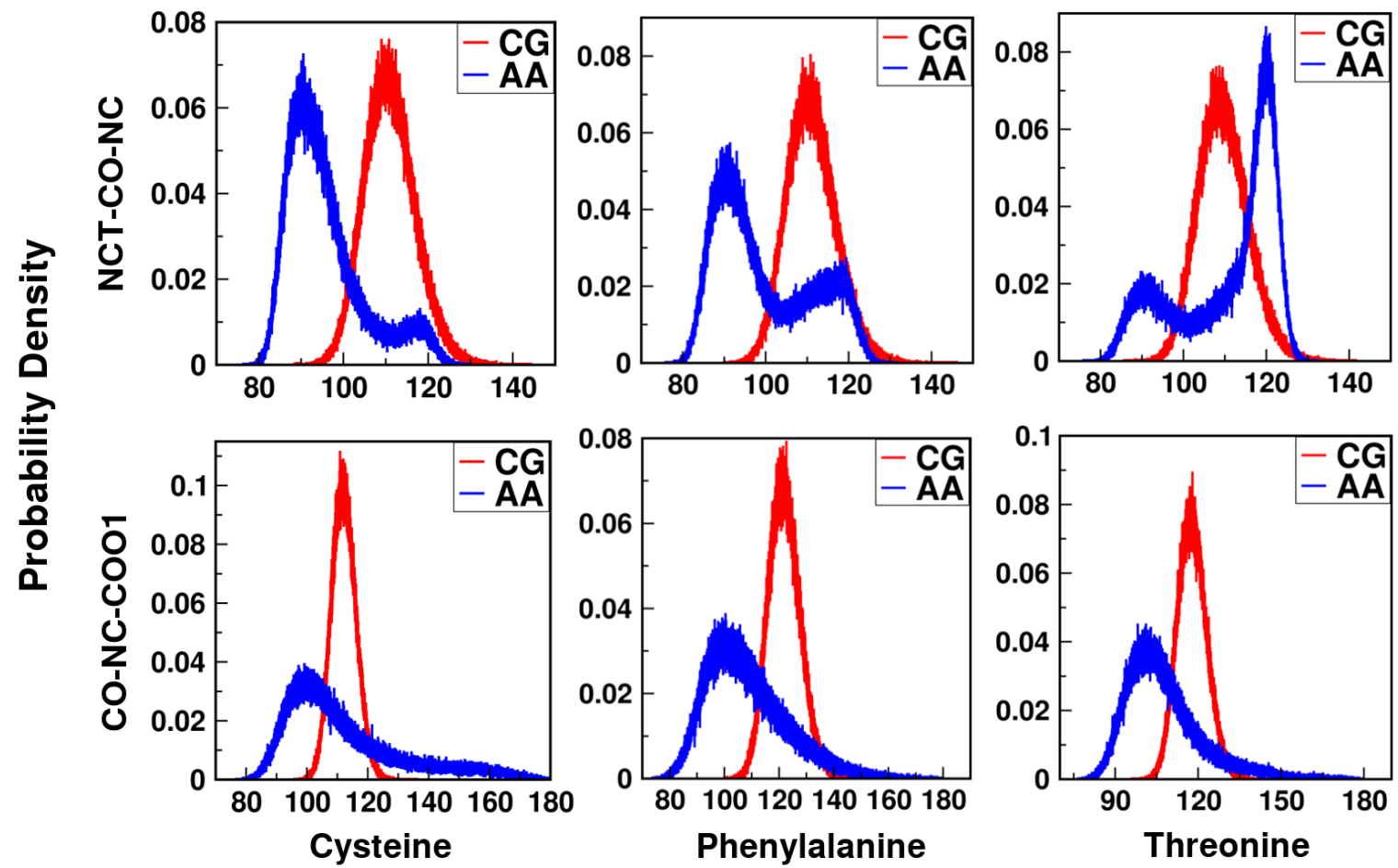

\section{Angle $\left({ }^{\circ}\right)$}

Figure 35: Comparison of backbone angle distributions for the angles NCT-CO-NC (top row) and CO-NC-COO1 (bottom row) from the cysteine (first column), phenylalanine (second column), and threonine (third column) dipeptides.
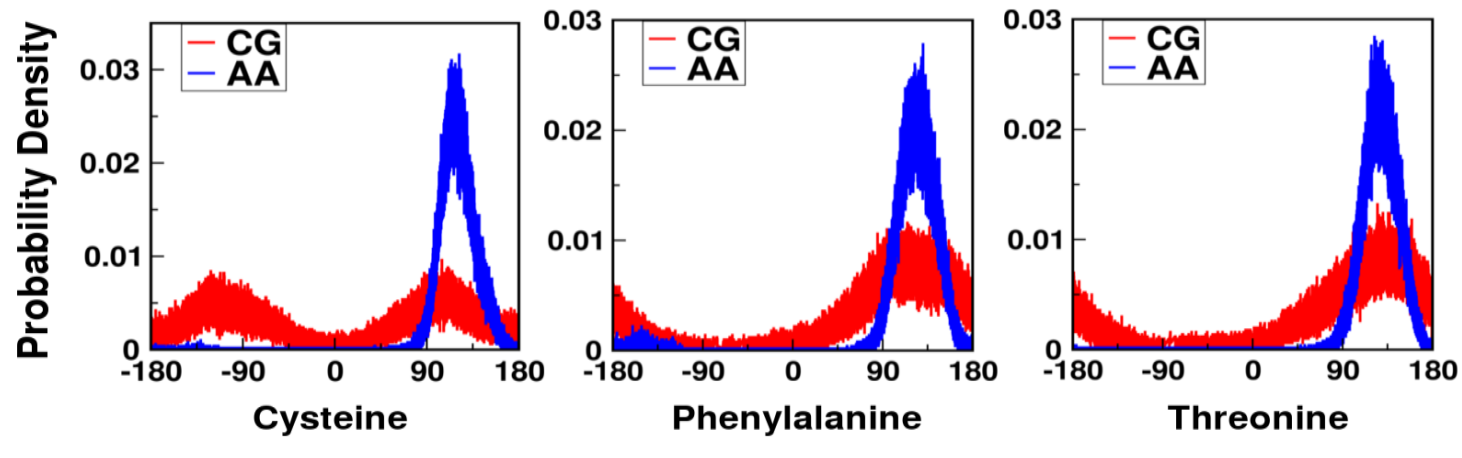

Dihedral $\left({ }^{\circ}\right)$

Figure 36: Comparison of backbone NCT-CO-NC-COO1 distribution from the cysteine (left), phenylalanine (center), and threonine (right) dipeptides.

The fourth issue of note is that some shifting of bonds and angles and some narrowing of

angle distributions were noticed in the CG distributions as compared with the AA distributions.

Upon investigation, it became apparent that the primary cause of this was from non-bonded

interactions of beads within a molecule that were within two or three bonds of each other, since we had only excluded interactions between beads directly bonded to each other ("exclude 1-2" in the 
configuration file). When excluding interactions between beads that were within two bonds of each other ("exclude 1-3"), the shifting and narrowing largely disappeared to allow much better overlap between the CG and AA distributions. This was also true of using "exclude scaled1-4" in the configuration file. Figures 37, 38, and 39 show examples of this from the leucine dipeptide; the bonded and non-bonded parameters used were the same for both CG models, but they do not represent the final parameters chosen for the CG model. Problematically, to use "exclude 1-3" or "exclude scaled1-4" often leads to greater instability in simulations and thus requires a smaller time step. Since, then, we were still able to maintain good overlap in many cases-as can be seen in the figures in the appendix — and only very little overlap in some, we decided to continue the parameterization with "exclude 1-2" at least to the point of attempting simulations of PA selfassembly using our CG models to obtain some indication of the amino acid bead and water bead interactions and how they might need to be adjusted. Later model development focusing on improving stability in the case of "exclude 1-3" or "exclude scaled1-4" could yield overall improvements in the model while enabling useful speed of simulations. Because the all-atom distributions have already been obtained, it would also be quite simple to generate the appropriate parameters for the case of "exclude 1-3" or "exclude scaled1-4." The non-bonded parameters would not need to be changed except for in two cases: 1) the CG bead representing the peptide backbone carbonyl, as that was parameterized based on a 3-bead mapping of diethylketone, and 2) the C2E2 bead for isoleucine as that was parameterized based on the four-bead mapping scheme shown in Figure 14(b). Some of the distributions (e.g. bond and angle distributions within ring structures such as that of the phenylalanine side chain) are not likely to differ based on the exclusion criteria. 

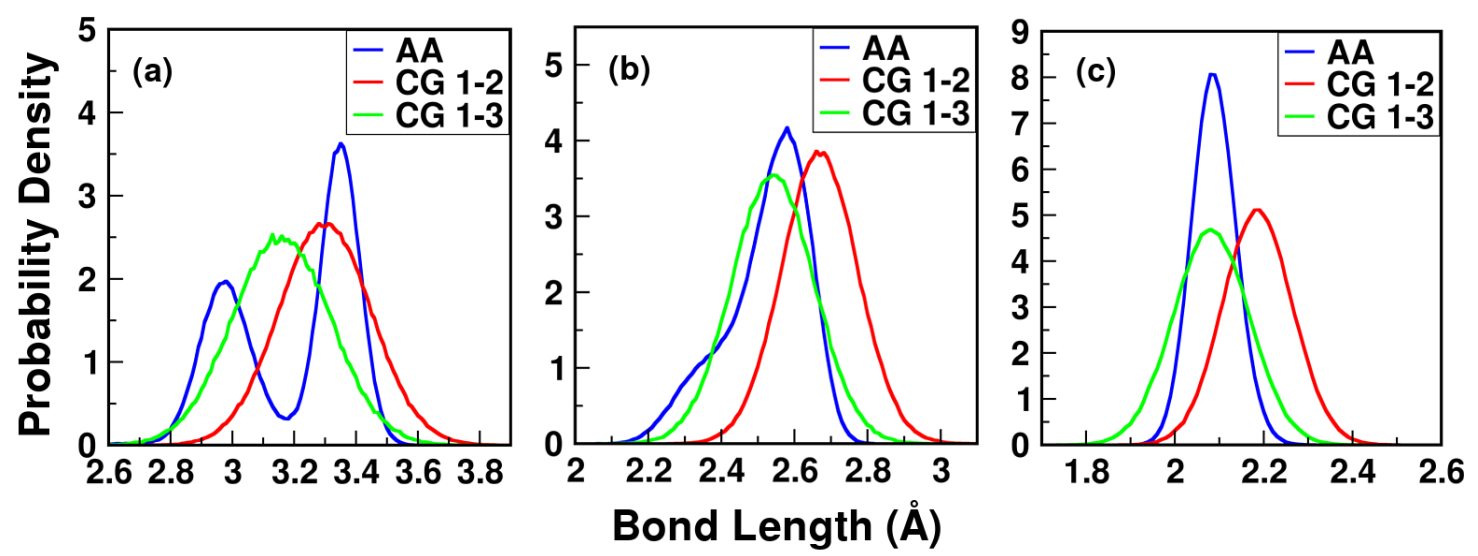

Figure 37: Comparisons of bond distributions, from the leucine dipeptide, between all-atom mapped (AA), coarse-grained using "exclude 1-2," (CG 1-2) and coarse-grained using "exclude 13" (CG 1-3). The same bonded and non-bonded parameters were used for both CG distributions. Distributions are of (a) C41-NCT, (b) NCT-CO, and (c) CO-NC.
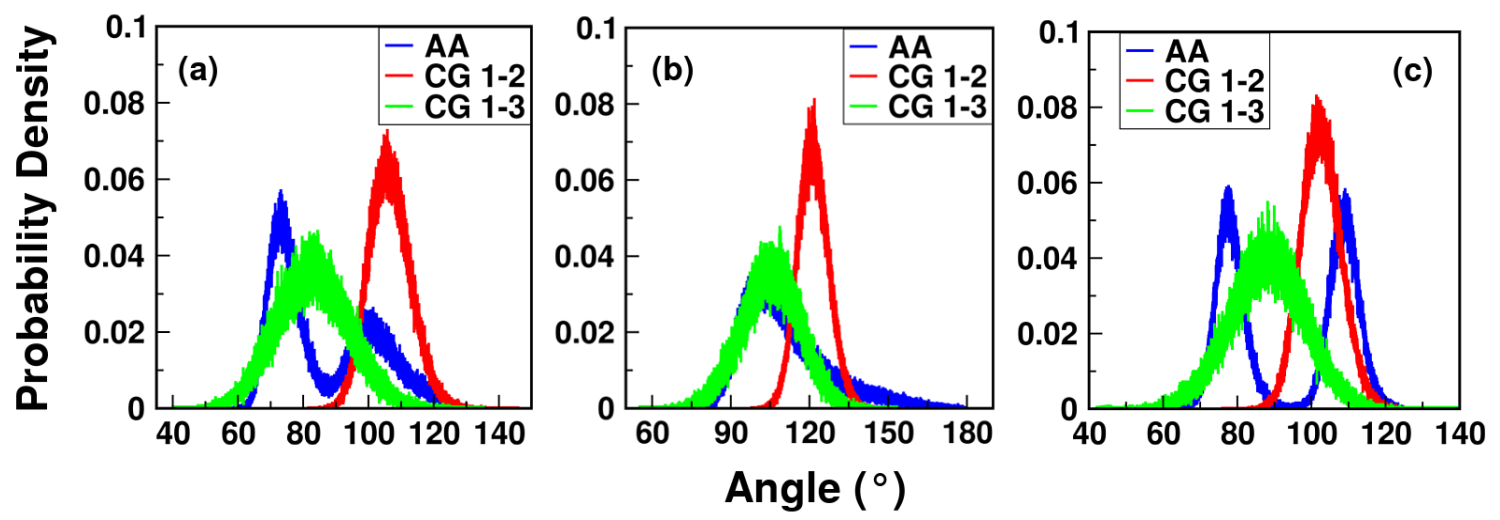

Figure 38: Comparisons of angle distributions, from the leucine dipeptide, between all-atom mapped (AA), coarse-grained using "exclude 1-2," (CG 1-2) and coarse-grained using "exclude 13" (CG 1-3). The same bonded and non-bonded parameters were used for both CG distributions. Distributions are of (a) C41-NCT-CO, (b) CO-NC-COO1, and (c) C41-NC-COO1.
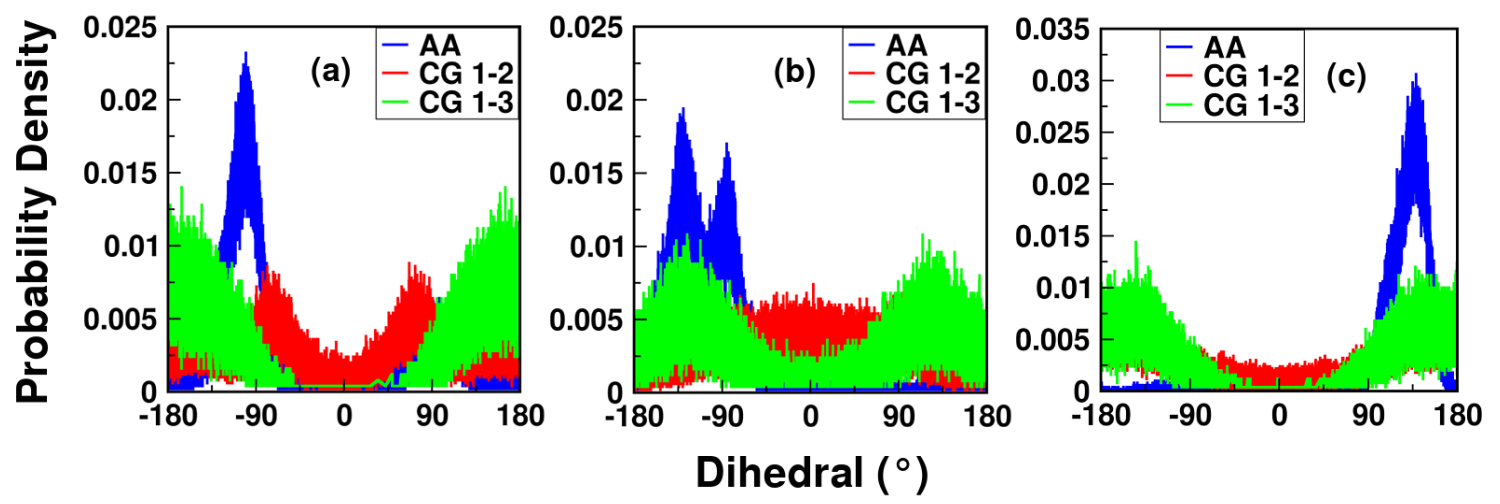

Figure 39: Comparisons of dihedral distributions, from the leucine dipeptide, between all-atom mapped (AA), coarse-grained using "exclude 1-2," (CG 1-2) and coarse-grained using "exclude 13" (CG 1-3). The same bonded and non-bonded parameters were used for both CG distributions. Distributions are of (a) C41-NCT-CO-NC, (b) NCT-CO-NC-C41, and (c) NCT-CO-NC-COO1. 
To test these hypotheses, the non-bonded parameters for the diethylketone beads were optimized under the "exclude 1-3" constraint. The optimized non-bonded parameters for the carbonyl bead were then plugged into glycine, histidine, alanine, and leucine CG dipeptide simulations to optimize the bonded parameters for those dipeptides under the "exclude 1-3" constraint. The densities of those simulations with optimized bonded and non-bonded parameters were then compared with the AA simulation densities of those dipeptides. The optimized nonbonded parameter values for the CO bead are given in Table 4. Table 5 shows the results of the CO bead parameterization with "exclude 1-2" (from a 100 ns simulation) versus "exclude 1-3" (from a 10 ns simulation; if the simulation were extended to $100 \mathrm{~ns}$, the results ought to be similar). Table 6 compares the density results for AA versus CG "exclude 1-2" versus CG "exclude 1-3" for the test dipeptides GlyGly, HisHis, AlaAla, and LeuLeu. Figure 40 compares the RDFs of the AA CG-mapped, CG "exclude 1-2," and CG "exclude 1-3" optimized CO bead in diethylketone (Figure 40(a)) and the leucine dipeptide (Figure 40(b)). Figures 41 and 42 provide examples of optimized bond and angle distributions, respectively, of the leucine dipeptide for the cases of CG "exclude 1-2" and CG "exclude 1-3" in comparison with the AA CG-mapped distributions. These distributions were improved in most cases. The poorness of the match in Figure 41(c) for CG 1-3 may be partly due to keeping the force constants low to enhance the stability. If the stability for CG 1-3 were able to be improved (this possibility will be discussed in Section 6 regarding future work), then possibly the force constant for that bond could be increased, leading to a better match.

Table 4: Optimized CO bead non-bonded parameters for CG "exclude 1-3" (CG 1-3) and CG "exclude 1-2" (CG 1-2)

\begin{tabular}{|c|c|c|}
\hline & CG 1-3 & CG 1-2 \\
\hline $\mathbf{r}_{\text {min }} / \mathbf{2}$ & 2.0967 & 2.4361 \\
\hline epsilon & -0.9472 & -0.6690 \\
\hline
\end{tabular}


Table 5: Comparisons of density, heat of vaporization, and surface tension results and errors for optimized CG with "exclude 1-3" parameterization (CG 1-3) and optimized CG with "exclude 1-2" parameterization (CG 1-2) diethylketone simulations with the experimental values (Exp.)

\begin{tabular}{|c|c|c|c|c|c|}
\hline & \multicolumn{3}{|c|}{ results } & \multicolumn{2}{c|}{ \% errors } \\
\hline & Exp. & CG 1-3 & CG 1-2 & CG 1-3 & CG 1-2 \\
\hline density & 0.8089 & 0.7908 & 0.7843 & 2.24 & 3.04 \\
\hline Hvap & 9.2065 & 8.8903 & 8.4050 & 3.43 & 8.71 \\
\hline Surface tension & 24.74 & 25.89 & 26.57 & 4.66 & 7.38 \\
\hline
\end{tabular}

Table 6: Density results for select dipeptide and tripeptide simulations: AA vs CG "exclude 1-3" (CG 1-3) vs CG "exclude 1-2" (CG 1-2). The CO bead as parameterized using CG "exclude 1-3" was used for all the $C G 1-3$ results.

\begin{tabular}{|l|l|l|l|l|l|}
\hline & \multicolumn{3}{|c|}{ results } & \multicolumn{2}{c|}{ \% errors } \\
\hline Peptide & \multicolumn{1}{|c|}{ AA } & \multicolumn{1}{|c|}{ CG 1-3 } & \multicolumn{1}{|c|}{ CG 1-2 } & \multicolumn{1}{c|}{ CG 1-3 } & CG 1-2 \\
\hline AlaAlaAla & 1.17043 & 1.35137 & 1.1685 & 15.46 & 0.16 \\
\hline GlyGly & 1.32142 & 1.32188 & 1.16645 & 0.03 & 11.73 \\
\hline HisHis & 1.33100 & 1.38957 & 1.2497 & 4.40 & 6.11 \\
\hline LeuLeu & 1.01118 & 1.08326 & 0.990916 & 7.13 & 2.00 \\
\hline
\end{tabular}
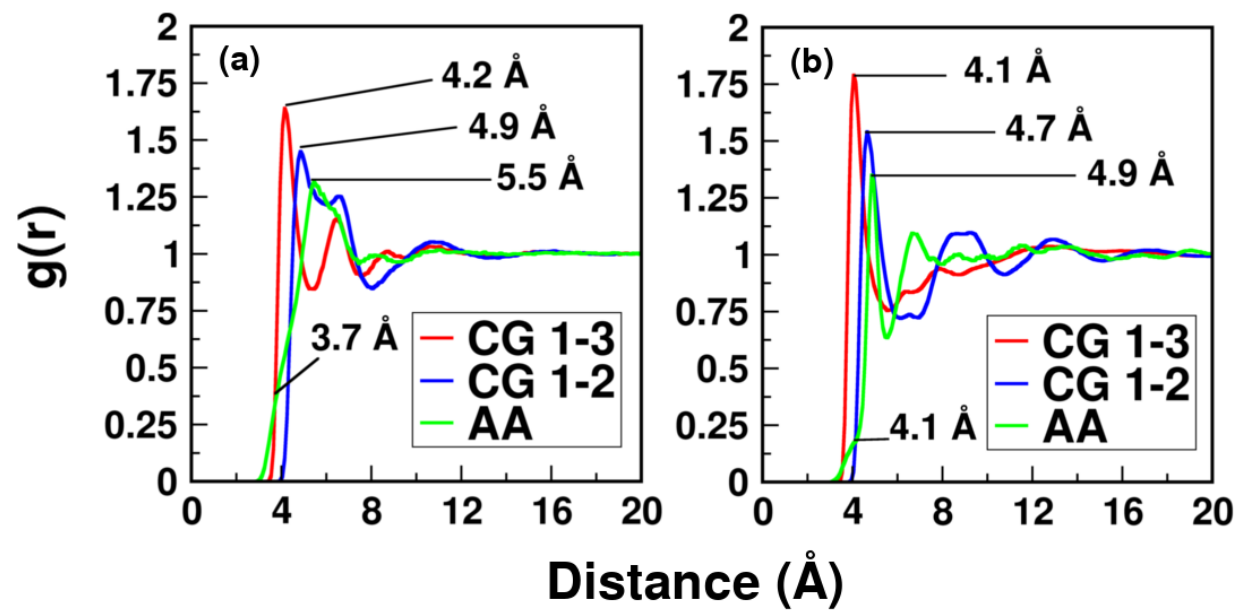

Figure 40: RDFs of the $C O$ bead in (a) diethylketone and (b) the leucine dipeptide for the AA CGmapped bead (AA), CG as parameterized with "exclude 1-2" (CG 1-2), and CG as parameterized with "exclude 1-3" (CG 1-3). 

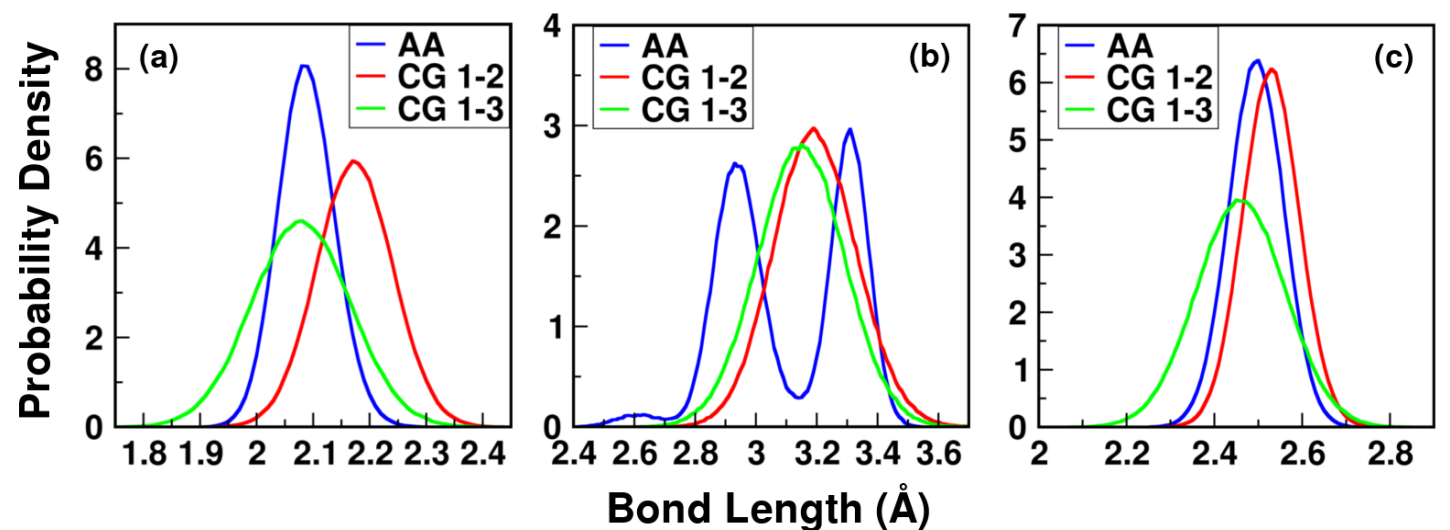

Figure 41: Distributions from the leucine dipeptide for the bonds (a) NCT-CO, (b) CO-NC, and (c) NC-C41 comparing the AA CG-mapped distribution (AA) with the CG distribution from parameterization with "exclude 1-2" (CG 1-2) and with the CG distribution from parameterization with "exclude 1-3" (CG 1-3).
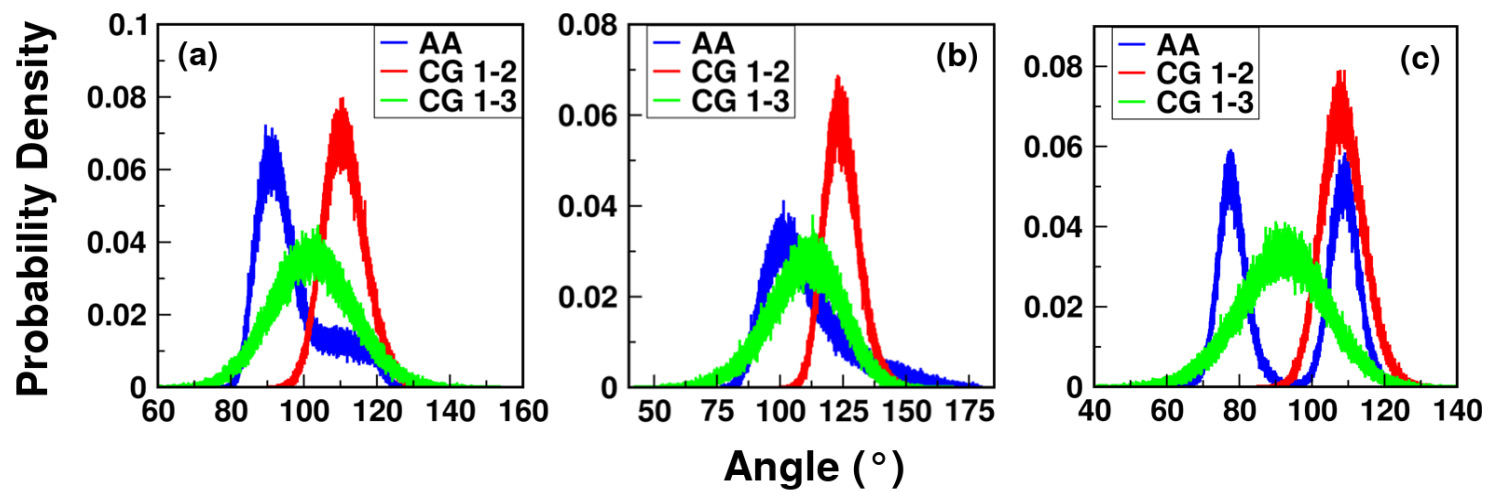

Figure 42: Distributions from the leucine dipeptide for the angles (a) NCT-CO-NC, (b) CO-NCCOO1, and (c) C41-NC-COO1 comparing the AA CG-mapped distribution (AA) with the CG distribution from parameterization with "exclude 1-2" (CG 1-2) and with the CG distribution from parameterization with "exclude 1-3" (CG 1-3).

Because of the challenges experienced with respect to "exclude 1-2" versus "exclude 1-3" or "exclude scaled1-4," alternatives to using diethylketone to parameterize the CO bead may lead to better outcomes. The most likely candidates include dimethylformamide and potentially Nmethylacetamide. Dimethylformamide has the advantage of a carbonyl bonded to an amide group, which is similar to the situation in a peptide backbone, although the amide of dimethylformamide is bonded to two carbons and no hydrogens rather than one carbon and one hydrogen as is the case in a peptide backbone; thus there is no potential for hydrogen bonding in dimethylformamide. Nmethylacetamide may be a possibility if the methyl group bonded to the carbonyl were to be modeled as a separate bead. However, the simulations would require a much smaller time step (e.g. 
1 or $2 \mathrm{fs}$ ) because of the smallness of the methyl bead. The advantage to using $\mathrm{N}$-methylacetamide, however, is that the backbone NC and backbone CO beads could be parameterized simultaneously, and the parameterization would include the effects of those beads in proximity to each other that is essentially the same as what occurs in a peptide backbone.

\section{PA Self-Assembly Studies}

\subsection{Initial Self-Assembly Studies}

As a first step in testing the amino acid models, we determined to attempt to simulate the self-assembly of a peptide amphiphile (PA). The PA chosen for this study was c16- $\mathrm{AHL}_{3} \mathrm{~K}_{3}-\mathrm{CO}_{2} \mathrm{H}$ shown in Figure 43; the CG model of the PA is shown in Figure 44. This is the PA that was simulated and synthesized in the study by Deshmukh, et al., ${ }^{4}$ examining the role of water in PA self-assembly. A combination of the Martini and CHARMM (with TIP3P water model) force fields were used for that study; the initial 150 ns of the simulation were with the CHARMM AA model which was mapped to the CG Martini model for the following $\sim 16 \mu \mathrm{s}$, and then at the end of the $16 \mu \mathrm{s}$, the system was back-mapped to the CHARMM model for a final 150 ns to show atomic-level details. The simulation included 300 PAs for a simulated duration of $16 \mu$ s at 1 bar and $340 \mathrm{~K}$. The PAs selfassembled into micelles in less than $1 \mu$ s which assembled into fibers within approximately 1.5-5 $\mu$ s with some ongoing fiber breakage and formation. The simulated micelles were comprised of approximately 50 PAs, and the simulated fibers were approximately $50 \AA ̊$ in diameter. These results served as a guide in our amino acid model development. 


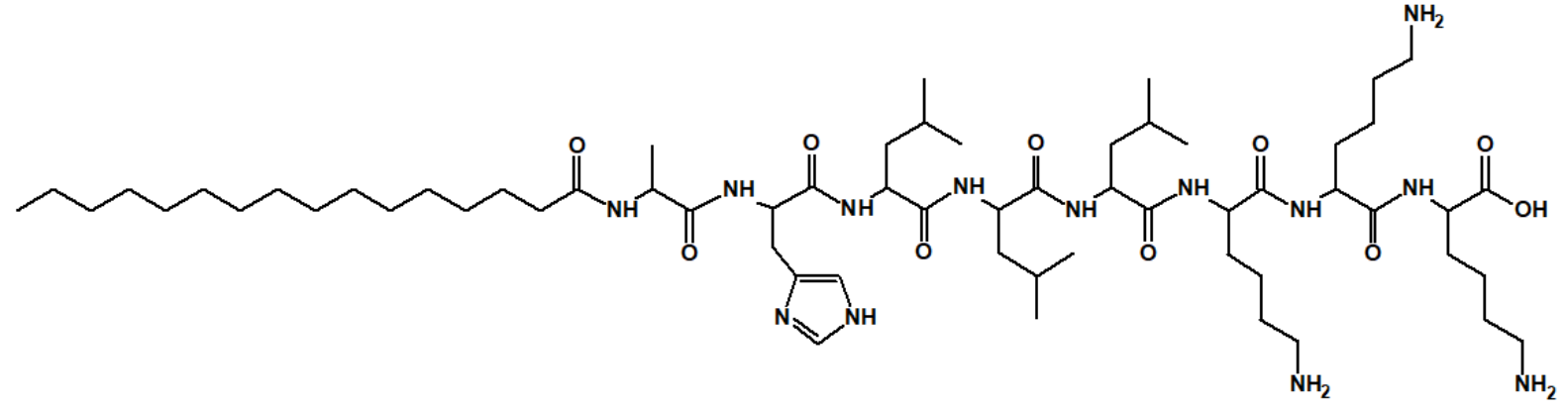

Figure 43: Structure of $\mathrm{c} 16-\mathrm{AHL}_{3} \mathrm{~K}_{3}-\mathrm{CO}_{2} \mathrm{H}$

In order to keep the required simulation time to a minimum while still enabling the possibility of PA fiber formation, we chose to model 150 PAs in an initial box size of 108 cubic $\AA$ with 16,000 of the 1 -site CG waters. ${ }^{33}$ These numbers are consistent with those used in other PA self-assembly studies. ${ }^{24,70}$ Periodicity of the MD simulations enabled the possibility of fiber formation from PAs on one side of the box interacting with the periodic images of PAs on the other side of the box. Simulations were conducted with NAMD and a pressure setpoint of 1 bar and a temperature setpoint of $340 \mathrm{~K}$ using the Nosé-Hoover Langevin piston barostat and the Langevin thermostat for pressure and temperature control. Intramolecular interactions were excluded for beads bonded to each other ("exclude 1-2"). A 12 Å cutoff was used with a switching distance of $9 \AA$ and a pairlist distance of $16 \AA$. Simulations were minimized for 10,000 steps and then conducted for a total of $15 \mu$ s with a 5 fs timestep.

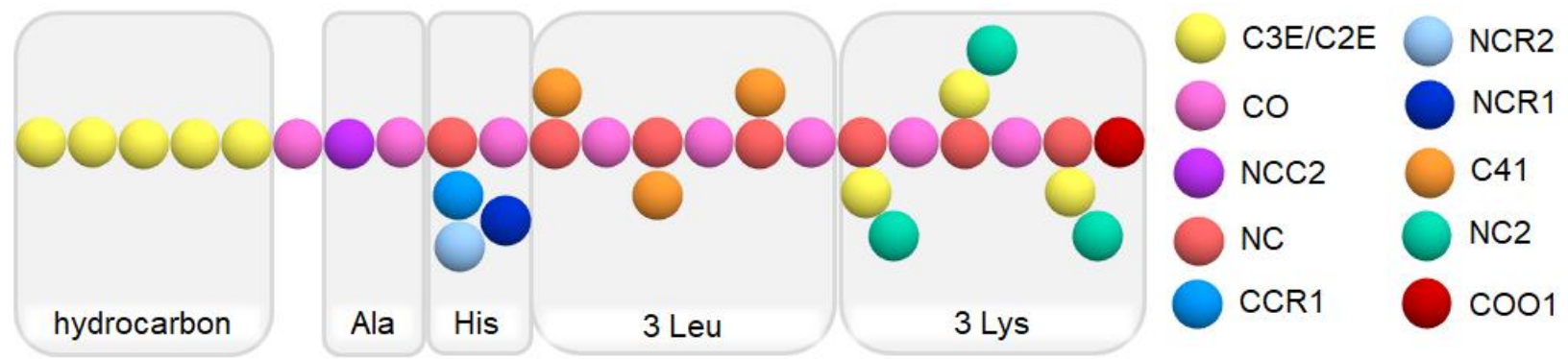

Figure 44: $\mathrm{CG}$ structure of $\mathrm{c} 16-\mathrm{AHL}_{3} \mathrm{~K}_{3}-\mathrm{CO}_{2} \mathrm{H}$ showing bead types

To begin with, we ran a PA simulation using the Lorentz-Berthelot (LB) combining rules for all the beads excepting the $\mathrm{C} 3 \mathrm{E}$ and $\mathrm{C} 2 \mathrm{E}$ beads which were given the interaction parameters with 
water that had previously been developed for them. ${ }^{48}$ As can be seen in Figure 44, the hydrocarbon tail was thus tuned with respect to water while the peptide portion primarily was not. The LB combining rules relate to the LJ functional form as shown in equation 21 in which $\varepsilon$ is the depth of the potential well, $\sigma$ is the distance at which the potential between particles is zero, and $r$ is the distance between particles. The Lorentz rule is given in equation 22, and the Berthelot rule is given in equation 23. After running the simulation for a few microseconds, however, it became apparent that defined, individual fibers were not forming. Within just a few nanoseconds, the PAs clumped together into micelle-like groups as shown in the 5 ns snapshot for the $100 \%$ simulation in Figure 46, in which the percentage reflects that the LB combining rules were not scaled for this simulation. Those micelle-like groups quickly bundled together to form windowpane structures which persisted throughout the $15 \mu$ s simulation, shown in Figure 46. Continually throughout the simulation, the assemblies broke apart (lost contiguity especially with respect to the hydrocarbon interiors) and regrouped. The $15 \mu$ s snapshot for the $100 \%$ simulation shows the PA assemblies in the middle of regrouping.

$$
\begin{gathered}
V_{L J}=4 \varepsilon\left[\frac{\sigma^{12}}{r}-\frac{\sigma^{6}}{r}\right] \\
\sigma_{i j}=\frac{\sigma_{i i}+\sigma_{j j}}{2} \\
\varepsilon_{i j}=\sqrt{\varepsilon_{i i} \varepsilon_{j j}}
\end{gathered}
$$

Because the $100 \%$ simulation was not behaving with the kind of character that experimental and MD studies indicate is typical for PA fiber self-assemblies, as described in Section 3.2, we decided to run two more simulations: one in which the epsilon interaction values were scaled to $85 \%$ of the combining rule values, and another in which the epsilon interaction values were scaled to $115 \%$ of the combining rule values. In neither case was the sigma interaction value scaled. This was because it is the epsilon value that controls the depth of the LJ potential well and 
describes the strength of attraction between two beads. In the case of the $115 \%$ simulation, the PAs showed clumping into micelle-like groups with the hydrocarbon tails bundling together. However, after more than a microsecond in simulation time, the PA peptide segments remained rather diffuse and showed no tendency to form fibers, as shown in Figure 45. Thus the strength of attraction of the peptide segment for the water beads was too strong. It also is unlikely that fibers would have formed, given the close proximity of the PAs to each other; if the peptide segments had preferred associating together rather than remaining somewhat diffuse in the water, the microsecond of simulation time provided ample opportunities to show such behavior. The $85 \%$ simulation, on the other hand, did show fiber formation. In this case, however, the fibers materialized immediately with no real micelle stage. Figure 46 shows that at just 5 ns, the PAs of the $85 \%$ simulation were already in a rough fiber formation. By the time $300 \mathrm{~ns}$ had been simulated, there were already neat fibers. These fibers remained stable throughout the remainder of the simulation. The measured diameter was approximately $58 \AA ̊$ at $15 \mu \mathrm{s}$.

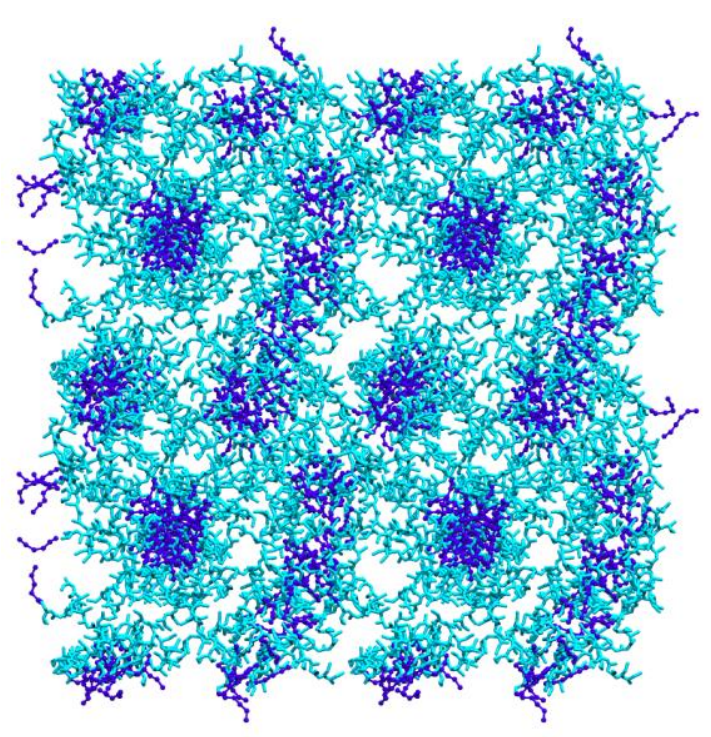

$115 \%-1.2 \mu s$

Figure 45: Simulation results after $1.2 \mu$ s with amino acid bead epsilon interaction values with water scaled to $115 \%$ of the combining rule values.

Because of the behavior exhibited with both the $100 \%$ and $85 \%$ simulations, we decided to run another simulation in which the epsilon interaction values were scaled to $90 \%$ of the 
combining rule values, and another in which the epsilon interaction values were scaled to $95 \%$ of the combining rule values. The $90 \%$ simulation results were very similar to the results of the $85 \%$ simulation, with no real micelle formation and essentially immediate fiber formation. The fiber diameter was measured at approximately $58 \AA$ at $15 \mu$ s in this case as well. The 95\% simulation behaved very similarly to the $100 \%$ simulation.

The formation of fibers with an increase in hydrophobicity makes sense considering that past research has shown the importance of hydrophobicity in fiber formation. ${ }^{27,28}$ However, the immediacy of formation and lack of a micelle stage suggest that the model may require further refinement, possibly by tuning the interaction parameters of different amino acid beads with each other. To help determine whether the scaled epsilon values were on the right track for the individual beads, hydration free energies were calculated for the beads, side chain groupings, or analogues, as described in the following paragraphs, for comparison with experimental values.

\subsection{Hydration Free Energies}

In order to provide insight into the realistic strength or weakness of the interactions between water and the amino acid beads utilized in the $\mathrm{c} 16-\mathrm{AHL}_{3} \mathrm{~K}_{3}-\mathrm{CO}_{2} \mathrm{H} \mathrm{PA}$, the potential of mean force (PMF) was calculated for water-solute systems in which the solute was representative of a bead or an analogue in the PA. In NAMD, it is relatively easy to calculate the PMF of a system using the adaptive biasing force $(A B F)$ method. To use this method to determine the Gibbs hydration free energy of a molecule, a 52 cubic Å box of 2000 CG waters was first equilibrated. Then a single solute molecule was placed approximately 20 Å outside the box in one dimension. The ABF will act along the reaction coordinate which, in our case, was defined as the projection in the z-direction of the distance between the center of mass of the water box and that of the solute molecule. The free energy along this coordinate can be considered as a potential that can be calculated from the average force acting along that coordinate, with the average force being the negative gradient of the potential (as in equation 3). Thus integrating the average force yields the potential. The 
instantaneous force that acts along the reaction coordinate is actually the sum of the average force-which only depends on the value of the coordinate-and a random force that has a zero average and which can be approximated as diffusive. The running time average of the instantaneous force is calculated during a simulation which provides an estimate of the derivative of the free energy at each point on the path that the solute molecule travels. At the same time that the average force is recorded, an equal and opposite external biasing force-the ABF-is applied so as to cancel that average force. This biasing force will stabilize at values close to zero when the average force is at an equilibrium value. At that point, there is an approximately flat potential of mean force which enables the solute molecule to easily move along and explore the coordinate when otherwise free energy barriers would have prohibited such exploration as the solute molecule would have gotten trapped in some states for lengths of time that could certainly exceed the duration of the simulation. ${ }^{71}$

In NAMD, the ABF method is implemented using the colvars package. ${ }^{72,73}$ Two windows were used: a window of $22 \AA$ to $40 \AA$ and another of $5 \AA$ to $22 \AA$; the water box was centered at $0 \AA$. Two windows were chosen in this manner to allow for sufficient exploration of the reaction coordinate for $50 \mathrm{~ns}$ simulations. For window 2 , the initial configuration file was taken from the window 1 configuration shortly after the simulation had begun and at a point at which the solute molecule had moved into the interface between the water and vacuum. The $50 \mathrm{~ns}$ simulation for each window was used to obtain the PMFs. CG amino acid side chain structures and analogues were used: N-butylamine for alanine, N-butylamine for lysine, 4-methylimidazole for histidine, isobutane for leucine, formaldehyde for the backbone carbonyl, and $\mathrm{N}$-methylacetamide for the backbone NC bead. The 1-site CG water model was used for the water. The combining rules were used for the amino acid bead and water bead interaction parameters, except for the C2 and C3 hydrocarbon beads for which the already-developed interaction parameters ${ }^{48}$ were used. Simulations were run at $298 \mathrm{~K}$ and 1 bar for comparison with experimental values at the same 
conditions. The results are shown in Figure 47 and Table 7. The differences in the N-butylamine results occur because of the different mapping schemes. These free energy calculations are a ballpark approximation to help indicate whether the amino acid bead interaction parameters with water are approximately correct, or in which direction they should move (higher for greater attraction to water, or lower for less attraction to water). The results indicate that the attraction of the amino acid beads for water is too strong for the leucine side chain bead, pretty decent for the CO bead (represented by formaldehyde), and not strong enough for the remainder. Considering these results in light of the PA simulation results, it seems likely that while the individual beads may experience stronger attractions to water than the combining rules provide for, there are also stronger interactions between different amino acid beads to each other than the combining rules provide. In order to achieve the hydrophobicity necessary for PA self-assembly, it may thus be necessary to consider the strength of the attractions between different amino acid beads.

Table 7: Experimental and CG MD values of hydration free energies, $\Delta G_{h y d}$, for select molecules.

\begin{tabular}{ccc}
\hline Molecule & $\begin{array}{c}\Delta G_{\text {hyd }}^{\text {exp }} \\
(\mathrm{kcal} / \mathrm{mol})\end{array}$ & $\begin{array}{c}\Delta G_{\text {hyd }}^{C G} \\
(\mathrm{kcal} / \mathrm{mol})\end{array}$ \\
\hline N-butylamine (Ala) & -9.52 & -3.0 \\
N-butylamine (Lys) & -9.52 & -0.4 \\
4-methylimidazole (His) & -10.27 & -7.0 \\
isobutane (Leu) & 2.28 & -1.0 \\
formaldehyde (CO) & -2.77 & -1.5 \\
N-methylacetamide (NC) & -10.07 & -5.0 \\
\hline a $\Delta G_{\text {hyd }}^{\text {exp }}$ values from refs $74-76$. & & \\
\hline
\end{tabular}



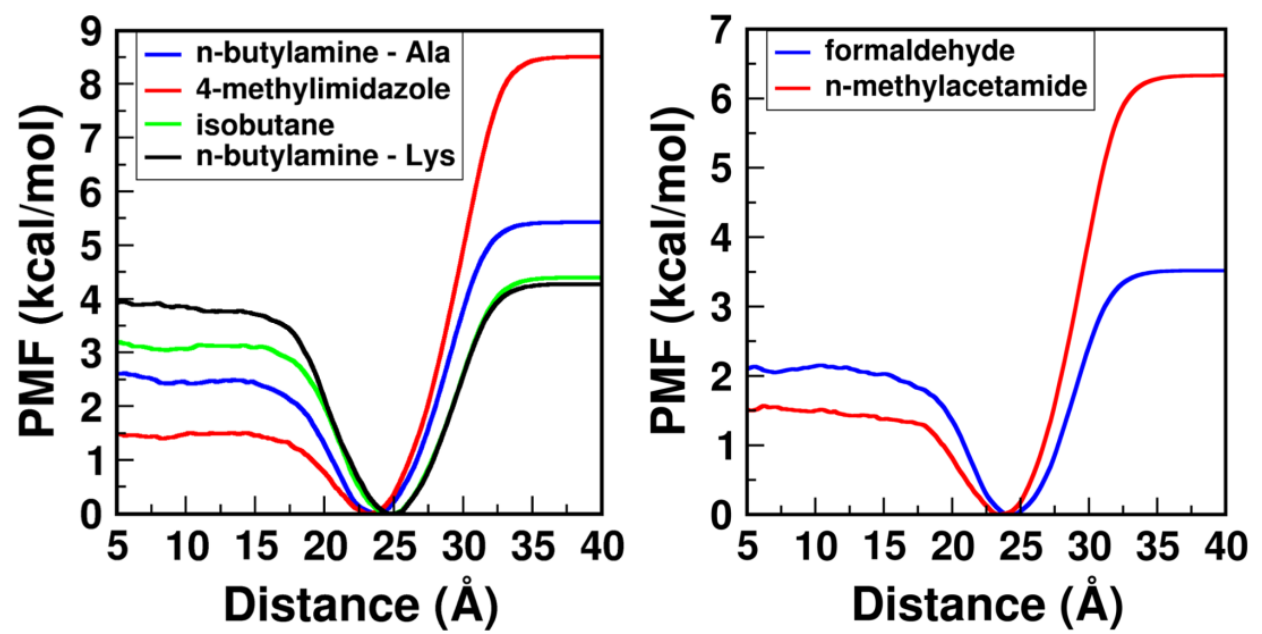

Figure 47: Free energy results for side chain and backbone CG structures and analogues

\subsection{Final Self-Assembly Study}

Because of the results from the hydration free energy studies, one more PA simulation was conducted. For this simulation, denoted "varied," in Figure 46, all the amino acid bead interaction parameters were scaled up to $115 \%$ of the combining rule values except for the CCR1 histidine bead which was neither scaled up nor down, the backbone carbonyl (CO) bead which was scaled up to $105 \%$ of the combining rule value, and the leucine side chain bead (C41) which was scaled down to $70 \%$ of the combining rule value in order to better approximate the epsilon interaction parameter of a hydrocarbon bead with water. The epsilon value of CCR1 was not scaled since it represents two $\mathrm{sp}^{2}$ hybridized carbons and no nitrogen or carbon atoms, and the CO bead was only scaled up slightly since the hydration free energy results appeared to indicate that the combining rule was approximately correct for the carbonyl.

This simulation in which scaling was varied has the advantage of showing micelle formation, but as seen in the $15 \mu$ s snapshot in Figure 46, the PAs remained as micelles and never formed fibers. Again, given the proximity of the PA micelles to each other, it seems unlikely that continuing the simulation for a longer period of time would have resulted in fiber formation. Additionally, research published using the Martini model to simulate the c16- $\mathrm{AHL}_{3} \mathrm{~K}_{3}-\mathrm{CO}_{2} \mathrm{H} \mathrm{PA}$ showed fiber formation within the first few microseconds, ${ }^{4}$ although it is possible that such a time 


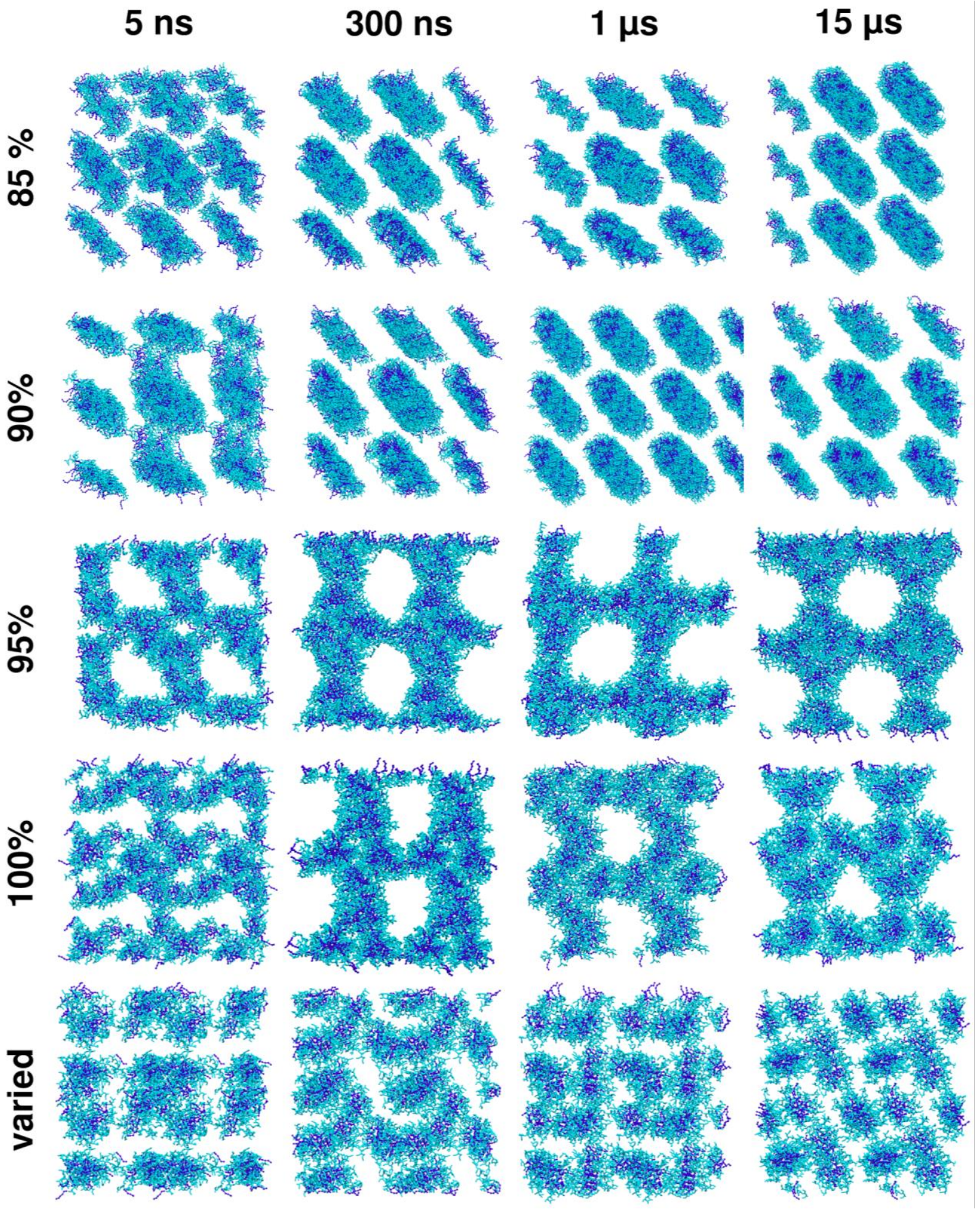

Figure 46: Snapshots from simulations of PAs (blue) in water (not shown). Darker blue - hydrocarbon tails; lighter blue - peptide segments. Percentages reflect percent of combining rule epsilon values while the "varied" simulation possessed primarily $115 \%$ of combining rule epsilon values with two bead types scaled to $70 \%$ and $105 \%$ and a third bead type left with its combining rule value. 
frame is not realistic. Considering that the $115 \%$ simulation shows clumping only of the hydrocarbon tails while the "varied" simulation shows more defined micelle formation, it becomes clear that it is important to consider the individual chemistry of each bead based on the atoms and functional group(s) the bead represents. This is especially clear considering the hydration free energy study results and when considering that the hydrocarbon leucine side chain bead (C41) certainly would interact with water differently than the beads which possess atoms with the capability of forming hydrogen bonds.

\section{Conclusions \& Future Work}

At the beginning of the project of which this manuscript provides a record, it was desired to 1) develop mapping schemes for the amino acids and peptide backbone with intermediate resolution (a preference for 2:1 and 3:1 beads), 2) optimize non-bonded parameters for the beads based on experimental thermodynamic values of analogues, 3) define appropriate bonded parameters for the amino acids and peptides based on all-atom dipeptide simulation bond, angle, and dihedral distributions, and 4) use the non-bonded and bonded parameters thus developed for c16- $\mathrm{AHL}_{3} \mathrm{~K}_{3}-\mathrm{CO}_{2} \mathrm{H}$ PA simulation studies with the goal of witnessing fiber formation as a way to test the model and provide an indication of the direction needed for future model development. The mapping scheme was defined without too much trouble; only in two cases (tryptophan and tyrosine) were we required to modify the mapping scheme for viability. The optimized non-bonded parameters that were developed provided, for the most part, good agreement with experimental results. Parameterization of methanol, ethanol, m-cresol, and diethylketone (with "exclude 1-2") partially fell short in this regard and may require more work and different tactics to attempt to improve. The bonded parameters developed resulted in CG bond, angle, and dihedral distributions that are shown throughout the Appendix. In some cases the matches were excellent, mostly they were reasonable, and in some cases there was little overlap between the CG and AA CG-mapped 
distributions. The reason for incidents of poor overlap, as discussed, was identified primarily as a result of the 1-2 exclusion criteria provided in the CG configuration file. This could easily be remedied, but with the catch that the result would be an overall much less stable model with the implication that it may not be feasible in some cases to conduct simulations-unless, of course, some other fix was found for stabilizing the model. With respect to the PA simulations, the results showed that use of the LB combining rules fails to capture the behavior expected based on experimental and MD research. When the overall hydrophobicity was increased, fibers formed; yet scaling down the interactions between the amino acid beads and water seemed, for the most part, the wrong direction to take based on the hydration free energies the beads exhibited in comparison with experimental values. Most likely, an overall increase in the hydrophobicity combined with sensitivity to the particular chemistry each bead represents would be required for more physically realistic models. This may be better accomplished by increasing the strength of attraction of some of the amino acid beads types for each other than by decreasing the strength of attraction of some of the amino acid bead types for the water bead. The hydration free energies may be useful in direction the scaling of the interaction parameters of the amino acid beads with the water bead. Obtaining guidance for tuning the interaction parameters of the amino acid beads with each other will be discussed below.

While this work represents great progress made in creating a model for amino acids and peptides, there are a number of challenges and limitations that this base model faces and which could be targeted for improvement. As previously noted at various points in this manuscript, these challenges and limitations include 1) lack of charges to represent charged amino acids; 2) the amino acid bead interactions have not been fine-tuned, and currently they rely on the LB combining rules, which may be inadequate in some cases; 3 ) simulations can be run at a 5 fs time step, but they are somewhat unstable and will frequently crash after only a few million steps; 4) no explicit modeling of hydrogen bonding and no attempts to parameterize for secondary structure 
conformations; and 5) our model shows that the bond and angle distributions do not always match the AA bond and angle distributions very well, with shifting and narrowing being observed in many cases. Potential solutions to these challenges and limitations will be addressed individually in the paragraphs below.

(1) The amino acids exist as zwitterions at neutral $\mathrm{pH}$, with a positive charge on the $\mathrm{N}$ terminus and a negative charge on the C-terminus. There are also various pKa values for many of the amino acid side chains, such as histidine and glutamic acid. While in laboratory experiments a buffer is sometimes added to neutralize an amino acid sequence, the reality in many cases is going to be that most or all of the amino acids that are capable of possessing a charge are going to possess a charge in the experimental conditions. For this reason, it is important for a protein force field to contain parameters for the amino acids in these charged states, as is the case with the AA force field CHARMM and the CG Martini and SDK force fields, for example. The charges in the SDK model were developed by parameterization against guanidium salt solutions at various concentrations, and the model does not distinguish lysine from arginine or aspartic acid from glutamic acid. ${ }^{77}$ The Martini model appears to have used AA simulations of the charged residues for parameterizing the charged beads. I suggest that for the model described in this manuscript, AA simulations of charged residues or analogues using the CHARMM force field be used to parameterize the beads that represent any atoms that could exist in a charged state.

(2) Tuning the interaction parameters of the amino acid beads with each other could be challenging. In the Martini model, amino acid beads were classified into four main types (polar, nonpolar, apolar, and charged) with sub-classifications based on hydrogen bonding capabilities or degree of polarity. Amino acid beads were assigned a classification based on water/oil partitioning coefficients of analogues of the side chains. The beads were then assigned interaction parameters with other types in accordance with the interaction matrix given in the original Martini publication. ${ }^{21}$ Something similar could be undertaken for the models developed in this manuscript. 
(3) As long as the model contains beads that contains only two heavy atoms (a 2:1 mapping scheme), that in and of itself will partially restrict the ability of the model to run smoothly without crashing at larger time steps, since the mass of a bead with a 2:1 mapping scheme is typically lower which influences the stability. ${ }^{49}$ Without artificially inflating the mass of a bead, an option that could potentially result in improved stability would be to fix to a given length the bonds of ring structures (as in the side chains of histidine, phenylalanine, tyrosine, and tryptophan). These bonds tend to have very small ranges over which they vibrate, as shown in Figure 33, instead of using a somewhat high force constant for those bonds, replacing the harmonic bonds and angles of the ring with a set of constraints could be a potential work-around. This is what was done in the Martini model. ${ }^{44}$ Additionally, a careful analysis of the angles and dihedrals could reveal situations in which heavy fluctuations are likely to happen (when three of the four beads in a dihedral have a very wide angle, close to 180 degrees). In that case, the options of removing any dihedrals deemed unnecessary or using special forms of dihedrals could be evaluated and implemented. ${ }^{78}$

(4) Tuning the amino acid bead interaction parameters with each other would be a way to capture the potential of hydrogen bond formation between particular amino acid beads. This may be especially important for the backbone $\mathrm{CO}$ and $\mathrm{NC}$ beads since it is the ability of the carbonyl oxygen to hydrogen bond with the hydrogen of the backbone nitrogen that leads to secondary structures like $\alpha$ helices and $\beta$ sheets.

(5) The shifts and narrowing of bond and angle distributions was shown to result from using "exclude 1-2" while the distributions were much improved when using "exclude 1-3" and "exclude scaled1-4." While the latter two tend to lead to greater instability and the requirement of a smaller time step than when applying "exclude 1-2," it could be an option to use "exclude 1-3" or "exclude scaled1-4" if any of the potential methods for stabilization-as addressed in (3) aboveare effective. 
Another possibility for future work is to conduct CG MD simulations for some of the analogues at different temperatures to test how well they reproduce experimental values (density, heat of vaporization, and surface tension) at the same temperatures. Depending on how well the models were able to reproduce the experimental values, re-parameterization could be considered to try to obtain a better fit across a temperature range if desired. Another option would be to define particular parameters for particular temperatures for overall accuracy for a wide range of conditions.

With such efforts directed towards improving the amino acid models, the work described in this manuscript has the potential to result in CG models ideal for studying PA self-assembly that, in combination with the hydrocarbon ${ }^{47}$ and water ${ }^{33}$ models previously developed by the group, may lead to more physically accurate simulations than other protein models currently available. Already, this work has shown great promise in this respect. And because of the potential for PAs to be useful in especially biomedical applications, such efforts may be quite rewarding. 


\section{References}

(1) Kokkoli, E.; Mardilovich, A.; Wedekind, A.; Rexeisen, E. L.; Garg, A.; Craig, J. A. Self-Assembly and Applications of Biomimetic and Bioactive Peptide-Amphiphiles. Soft Matter 2006, 2 (12), 1015-1024. https://doi.org/10.1039/b608929a.

(2) Cui, H.; Webber, M. J.; Stupp, S. I. Self-Assembly of Peptide Amphiphiles: From Molecules to Nanostructures to Biomaterials. Biopolymers 2010, 94 (1), 1-18.

https://doi.org/10.1002/bip.21328.

(3) Eskandari, S.; Guerin, T.; Toth, I.; Stephenson, R. J. Recent Advances in Self-Assembled Peptides: Implications for Targeted Drug Delivery and Vaccine Engineering. Adv. Drug Deliv. Rev. 2017, 110-111, 169-187. https://doi.org/10.1016/J.ADDR.2016.06.013.

(4) Deshmukh, S. A.; Solomon, L. A.; Kamath, G.; Christopher Fry, H.; S Sankaranarayanan, S. K. Water Ordering Controls the Dynamic Equilibrium of Micelle-Fibre Formation in SelfAssembly of Peptide Amphiphiles. Nat. Commun. 2016, 7, 1-11. https://doi.org/10.1038/ncomms12367.

(5) Brini, E.; Algaer, E. A.; Ganguly, P.; Li, C.; Rodríguez-Ropero, F.; Van Der Vegt, N. F. A. Systematic Coarse-Graining Methods for Soft Matter Simulations-a Review. Soft Matter 2013, 9 (7), 2108-2119. https://doi.org/10.1039/c2sm27201f.

(6) Kmiecik, S.; Gront, D.; Kolinski, M.; Wieteska, L.; Dawid, A. E.; Kolinski, A. Coarse-Grained Protein Models and Their Applications. Chem. Rev. 2016, 116 (14), 7898-7936. https://doi.org/10.1021/acs.chemrev.6b00163.

(7) Ingólfsson, H. I.; Lopez, C. A.; Uusitalo, J. J.; de Jong, D. H.; Gopal, S. M.; Periole, X.; Marrink, S. J. The Power of Coarse Graining in Biomolecular Simulations. Wiley Interdiscip. Rev. Comput. Mol. Sci. 2014, 4 (3), 225-248. https://doi.org/10.1002/wcms.1169.

(8) Berg JM, Tymoczko JL, S. L. Biochemistry, 5th ed.; W. H. Freeman: New York, 2002.

(9) Biology 2e, 2nd ed.; OpenStax CNX, 2018.

(10) Boekhoven, J.; Zha, R. H.; Tantakitti, F.; Zhuang, E.; Zandi, R.; Newcomb, C. J.; Stupp, S. I. Alginate-Peptide Amphiphile Core-Shell Microparticles as a Targeted Drug Delivery System. RSC Adv. 2015, 5 (12), 8753-8756. https://doi.org/10.1039/C4RA16593D.

(11) Bulut, S.; Erkal, T. S.; Toksoz, S.; Tekinay, A. B.; Tekinay, T.; Guler, M. O. Slow Release and Delivery of Antisense Oligonucleotide Drug by Self-Assembled Peptide Amphiphile Nanofibers. Biomacromolecules 2011, 12 (8), 3007-3014. https://doi.org/10.1021/bm200641e.

(12) Silva, G. A.; Czeisler, C.; Niece, K. L.; Beniash, E.; Harrington, D. A.; Kessler, J. A.; Stupp, S. I. Selective Differentiation of Neural Progenitor Cells by High-Epitope Density Nanofibers. Science (80-. ). 2004, 303 (5662), 1352-1355. https://doi.org/10.1126/SCIENCE.1093783.

(13) Tysseling-Mattiace, V. M.; Sahni, V.; Niece, K. L.; Birch, D.; Czeisler, C.; Fehlings, M. G.; Stupp, S. I.; Kessler, J. A. Self-Assembling Nanofibers Inhibit Glial Scar Formation and Promote Axon Elongation after Spinal Cord Injury. J. Neurosci. 2008, 28 (14), 3814-3823. https://doi.org/10.1523/JNEUROSCI.0143-08.2008. 
(14) Qian, Y.; Kaur, K.; Foster, J. C.; Matson, J. B. Supramolecular Tuning of $\mathrm{H}_{2} \mathrm{~S}$ Release from Aromatic Peptide Amphiphile Gels: Effect of Core Unit Substituents. Biomacromolecules 2019, 20 (2), 1077-1086. https://doi.org/10.1021/acs.biomac.8b01732.

(15) Matsui, H.; MacCuspie, R. Metalloporphyrin Nanotube Fabrication Using Peptide Nanotubes as Templates. Nano Lett. 2001, 1 (12), 671-675. https://doi.org/10.1021/NL0156080.

(16) Fry, H. C.; Garcia, J. M.; Medina, M. J.; Ricoy, U. M.; Gosztola, D. J.; Nikiforov, M. P.; Palmer, L. C.; Stupp, S. I. Self-Assembly of Highly Ordered Peptide Amphiphile Metalloporphyrin Arrays. J. Am. Chem. Soc. 2012, 134 (36), 14646-14649. https://doi.org/10.1021/ja304674d.

(17) Garifullin, R.; Erkal, T. S.; Tekin, S.; Ortaç, B.; Gürek, A. G.; Ahsen, V.; Yaglioglu, H. G.; Elmali, A.; Guler, M. O. Encapsulation of a Zinc Phthalocyanine Derivative in Self-Assembled Peptide Nanofibers. J. Mater. Chem. 2012, 22 (6), 2553-2559. https://doi.org/10.1039/C1JM14181C.

(18) Huang J, M. A. J. CHARMM36 All-Atom Additive Protein Force Field: Validation Based on Comparison to NMR Data. J. Comput. Chem. 2013, 34 (25), 2135-2145.

https://doi.org/10.1002/jcc.23354.

(19) Cornell, W. D.; Cieplak, P.; Bayly, C. I.; Gould, I. R.; Merz, K. M.; Ferguson, D. M.; Spellmeyer, D. C.; Fox, T.; Caldwell, J. W.; Kollman, P. A. A Second Generation Force Field for the Simulation of Proteins, Nucleic Acids, and Organic Molecules. J. Am. Chem. Soc. 1995, 117 (19), 51795197. https://doi.org/10.1021/ja00124a002.

(20) Robertson, M. J.; Tirado-Rives, J.; Jorgensen, W. L. Improved Peptide and Protein Torsional Energetics with the OPLS-AA Force Field. J. Chem. Theory Comput. 2015, 11 (7), 3499-3509. https://doi.org/10.1021/acs.jctc.5b00356.

(21) Monticelli, L.; Kandasamy, S. K.; Periole, X.; Larson, R. G.; Peter, D.; Marrink, S.-J. The MARTINI Coarse-Grained Force Field: Extension to Proteins. 2008, 819-834. https://doi.org/10.1021/ct700324x.

(22) Cheon, M.; Chang, I.; Hall, C. K. Extending the PRIME Model for Protein Aggregation to All 20 Amino Acids. Proteins Struct. Funct. Bioinforma. 2010, 78 (14), 2950-2960. https://doi.org/10.1002/prot.22817.

(23) Devane, R.; Shinoda, W.; Moore, P. B.; Klein, M. L.; Pennsyl, V. Transferable Coarse Grain Nonbonded Interaction Model for Amino Acids. J. Chem. Theory Comput. 2009, 5, 2115-2124.

(24) Lee, O. S.; Cho, V.; Schatz, G. C. Modeling the Self-Assembly of Peptide Amphiphiles into Fibers Using Coarse-Grained Molecular Dynamics. Nano Lett. 2012, 12 (9), 4907-4913. https://doi.org/10.1021/nl302487m.

(25) Shimada, T.; Sakamoto, N.; Motokawa, R.; Koizumi, S.; Tirrell, M. Self-Assembly Process of Peptide Amphiphile Worm-Like Micelles. J. Phys. Chem. B 2012, 116 (1), 240-243. https://doi.org/10.1021/jp209105z.

(26) Yu, T.; Schatz, G. C. Free-Energy Landscape for Peptide Amphiphile Self-Assembly: Stepwise versus Continuous Assembly Mechanisms. J. Phys. Chem. B 2013, 117 (45), 14059-14064. https://doi.org/10.1021/jp409305e. 
(27) Paramonov, S. E. .; Jun, H.-W.; Hartgerink, J. D. Self-Assembly of Peptide-Amphiphile Nanofibers: The Roles of Hydrogen Bonding and Amphiphilic Packing. J. Am. Chem. Soc. 2006, 128 (22), 7291-7298. https://doi.org/10.1021/JA060573X.

(28) Velichko, Y. S. .; Stupp, Samuel I.; Olvera de laCruz, M. Molecular Simulation Study of Peptide Amphiphile Self-Assembly. J. Phys. Chem. B 2008, 112 (8), 2326-2334.

https://doi.org/10.1021/JP074420N.

(29) Lee, O.-S.; Stupp, S. I.; Schatz, G. C. Atomistic Molecular Dynamics Simulations of Peptide Amphiphile Self-Assembly into Cylindrical Nanofibers. J. Am. Chem. Soc 2011, 133, 36773683. https://doi.org/10.1021/ja110966y.

(30) Ortony, J. H.; Newcomb, C. J.; Matson, J. B.; Palmer, L. C.; Doan, P. E.; Hoffman, B. M.; Stupp, S. I. Internal Dynamics of a Supramolecular Nanofibre. Nat. Mater. 2014, 13 (8), 812-816. https://doi.org/10.1038/nmat3979.

(31) Frederix, P. W. J. M.; Patmanidis, I.; Marrink, S. J. Molecular Simulations of Self-Assembling Bio-Inspired Supramolecular Systems and Their Connection to Experiments. Chem. Soc. Rev. 2018, 47 (10), 3470-3489. https://doi.org/10.1039/c8cs00040a.

(32) Manandhar, A.; Kang, M.; Chakraborty, K.; Tang, P. K.; Loverde, S. M. Molecular Simulations of Peptide Amphiphiles. Org. Biomol. Chem. 2017, 15 (38), 7993-8005.

https://doi.org/10.1039/C70B01290J.

(33) Bejagam, K. K.; Singh, S.; An, Y.; Berry, C.; Deshmukh, S. A. PSO-Assisted Development of New Transferable Coarse-Grained Water Models. J. Phys. Chem. B 2018, 122 (6), 1958-1971. https://doi.org/10.1021/acs.jpcb.7b10542.

(34) Davtyan, A.; Schafer, N. P.; Zheng, W.; Clementi, C.; Wolynes, P. G.; Papoian, G. A. AWSEM-MD: Protein Structure Prediction Using Coarse-Grained Physical Potentials and Bioinformatically Based Local Structure Biasing. J. Phys. Chem. B 2012, 116 (29), 8494-8503. https://doi.org/10.1021/jp212541y.

(35) Bereau, T.; Deserno, M. Generic Coarse-Grained Model for Protein Folding and Aggregation. J. Chem. Phys. 2009, 130 (23). https://doi.org/10.1063/1.3152842.

(36) Kolinski, A. Protein Modeling and Structure Prediction with a Reduced Representation. Acta Biochim. Pol. 2004, 51 (2), 349-371. https://doi.org/035001349.

(37) Hills, R. D.; Lu, L.; Voth, G. A. Multiscale Coarse-Graining of the Protein Energy Landscape. PLoS Comput. Biol. 2010, 6 (6), 1-15. https://doi.org/10.1371/journal.pcbi.1000827.

(38) Sterpone, F.; Melchionna, S.; Tuffery, P.; Pasquali, S.; Mousseau, N.; Cragnolini, T.; Chebaro, Y.; St-Pierre, J. F.; Kalimeri, M.; Barducci, A.; et al. The OPEP Protein Model: From Single Molecules, Amyloid Formation, Crowding and Hydrodynamics to DNA/RNA Systems. Chem. Soc. Rev. 2014, 43 (13), 4871-4893. https://doi.org/10.1039/c4cs00048j.

(39) Pasi, M.; Lavery, R.; Ceres, N. PaLaCe: A Coarse-Grain Protein Model for Studying Mechanical Properties. J. Chem. Theory Comput. 2013, 9 (1), 785-793. https://doi.org/10.1021/ct3007925. 
(40) Kar, P.; Gopal, S. M.; Cheng, Y. M.; Predeus, A.; Feig, M. PRIMO: A Transferable Coarse-Grained Force Field for Proteins. J. Chem. Theory Comput. 2013, 9 (8), 3769-3788. https://doi.org/10.1021/ct400230y.

(41) Das, R.; Baker, D. Macromolecular Modeling with Rosetta. Annu. Rev. Biochem. 2008, 77 (1), 363-382. https://doi.org/10.1146/annurev.biochem.77.062906.171838.

(42) Liwo, A.; Baranowski, M.; Czaplewski, C.; Gołaś, E.; He, Y.; Jagieła, D.; Krupa, P.; Maciejczyk, M.; Makowski, M.; Mozolewska, M. A.; et al. A Unified Coarse-Grained Model of Biological Macromolecules Based on Mean-Field Multipole-Multipole Interactions. J. Mol. Model. 2014, 20 (8), 2306. https://doi.org/10.1007/s00894-014-2306-5.

(43) Basdevant, N.; Borgis, D.; Ha-Duong, T. Modeling Protein-Protein Recognition in Solution Using the Coarse-Grained Force Field SCORPION. J. Chem. Theory Comput. 2013, 9 (1), 803813. https://doi.org/10.1021/ct300943w.

(44) Marrink, S. J.; Risselada, H. J.; Yefimov, S.; Tieleman, D. P.; De Vries, A. H. The MARTINI Force Field: Coarse Grained Model for Biomolecular Simulations. J. Phys. Chem. B 2007, 111 (27), 7812-7824. https://doi.org/10.1021/jp071097f.

(45) Uusitalo, Jaakko J.; Ingólfsson, Helgi I.; Akhshi, Parisa; Tieleman, D. Peter; Marrink, S. J. Martini Coarse-Grained Force Field: Extension to DNA. J. Chem. Theory Comput. 2015, 11 (8), 3932-3945. https://doi.org/10.1016/j.bpj.2017.05.043.

(46) Kang, M.; Cui, H.; Loverde, S. M. Coarse-Grained Molecular Dynamics Studies of the Structure and Stability of Peptide-Based Drug Amphiphile Filaments. Soft Matter 2017, 13 (42), 77217730. https://doi.org/10.1039/C7SM00943G.

(47) An, Y.; Bejagam, K. K.; Deshmukh, S. A. Development of New Transferable Coarse-Grained Models of Hydrocarbons. J. Phys. Chem. B 2018, 122 (28), 7143-7153. https://doi.org/10.1021/acs.jpcb.8b03822.

(48) An, Y.; Bejagam, K. K.; Deshmukh, S. A. Development of Transferable Nonbonded Interactions between Coarse-Grained Hydrocarbon and Water Models. J. Phys. Chem. B 2019, 123 (4), 909-921. https://doi.org/10.1021/acs.jpcb.8b07990.

(49) Choe, J. I.; Kim, B. Determination of Proper Time Step for Molecular Dynamics Simulation. Bull. Korean Chem. Soc. 2000, 21 (4), 419-424.

(50) Fitch, C. A.; Platzer, G.; Okon, M.; Garcia-Moreno, B. E.; McIntosh, L. P. Arginine: Its PKa Value Revisited. Protein Sci. 2015, 24 (5), 752-761. https://doi.org/10.1002/pro.2647.

(51) Frisch, M. J. et al. Gaussian 09 Revision D.01. Gaussian Inc.: Wallingford, CT 2009.

(52) Richard, A. M. and M. W. DSSTox (Distributed Structure-Searchable Toxicity Database Network) Project And Website. Presented at Board of Scientific Counselors 2005, RTP, NC, June 19 - 20, 2006.

(53) Kim S, Chen J, Cheng T, Gindulyte A, He J, He S, Li Q, Shoemaker BA, Thiessen PA, Yu B, Zaslavsky L, Zhang J, B. E. PubChem 2019 Update: Improved Access to Chemical Data. Nucleic Acids Res. 2019. https://doi.org/10.1093/nar/gky1033.

(54) Avogadro: An Open-Source Molecular Builder and Visualization Tool. 
(55) L. Martínez, R. Andrade, E. G. Birgin, J. M. M. Packmol: A Package for Building Initial Configurations for Molecular Dynamics Simulations. J. Comput. Chem. 2009, 30 (13), 21572164.

(56) Phillips, J. C.; Braun, R.; Wang, W.; Gumbart, J.; Tajkhorshid, E.; Villa, E.; Chipot, C.; Skeel, R. D.; Kalé, L.; Schulten, K. Scalable Molecular Dynamics with NAMD. J. Comput. Chem. 2005, 26 (16), 1781-1802. https://doi.org/10.1002/jcc.20289.

(57) Humphrey, W., Dalke, A. and Schulten, K. VMD - Visual Molecular Dynamics. J. Mol. Graph. 1996, 14, 33-38.

(58) Toton, D.; Lorenz, C. D.; Rompotis, N.; Martsinovich, N.; Kantorovich, L. Temperature Control in Molecular Dynamic Simulations of Non-Equilibrium Processes. J. Phys. Condens. Matter 2010, 22 (7), 074205. https://doi.org/10.1088/0953-8984/22/7/074205.

(59) Di Pierro, M.; Elber, R.; Leimkuhler, B. A Stochastic Algorithm for the Isobaric-Isothermal Ensemble with Ewald Summations for All Long Range Forces. J. Chem. Theory Comput. 2015, 11 (12), 5624-5637. https://doi.org/10.1021/acs.jctc.5b00648.

(60) Chandler, D. Introduction to Modern Statistical Mechanics; Oxford University Press: New York, 1987.

(61) Abadi, M.; Agarwal, A.; Barham, P.; Brevdo, E.; Chen, Z.; Citro, C.; Corrado, G. S.; Davis, A.; Dean, J.; Devin, M.; et al. TensorFlow: Large-Scale Machine Learning on Heterogeneous Distributed Systems. 2016.

(62) Wang, J.; Hou, T. Application of Molecular Dynamics Simulations in Molecular Property Prediction . 1. Density and Heat of Vaporization. J. Chem. Theory Comput. 2011, 7, 21512165. https://doi.org/10.1021/ct200142z.

(63) Yaws, C. L. Yaws' Handbook of Thermodynamic and Physical Properties of Chemical Compounds; Knovel, 2003.

(64) Shukla, R. K.; Kumar, A.; Srivastava, U.; Awasthi, N.; Pandey, J. D. Critical Evaluation of Surface Tension of Binary Liquid Mixtures from Associated and Nonassociated Processes at Various Temperatures: An Experimental and Theoretical Study. Can. J. Phys. 2013, 91 (3), 211-220.

(65) Diky, Vladimir; Muzny, Chris D.; Smolyanitsky, Alexander Y.; Bazyleva, Ala; Chirico, Robert D.; Magee, Joe W.; Paulechka, Yauheni; Kazakov, Andrei F.; Townsend, Scott A.; Lemmon, Eric W.; Frenkel, Michael D.; Kroenlein, K. G. NIST Standard Reference Database 103b. Thermodynamic Data for Pure Compounds. 2016.

(66) U.S. Coast Guard Department of Transportation. CHRIS - Hazardous Chemical Data. Volume II.; U.S. Government Printing Office: Washington, D.C., 1984.

(67) Horstmann, S.; Fischer, K.; Gmehling, J. Vapor-Liquid-Liquid Equilibria, Azeotropic, and Excess Enthalpy Data for the Binary System n-Undecane + Propionamide and PureComponent Vapor Pressure and Density Data for Propionamide. J. Chem. Eng. Data 2004, 49 (6), 1494-1498. https://doi.org/10.1021/je034255e.

(68) CRC Handbook of Chemistry and Physics, 94th ed.; Haynes, William M.; Lide, David R.; Bruno, T. J., Ed.; CRC Press: Boca Raton, 2013. 
(69) Wolfarth, Ch; Wolfarth, B. Surface Tension of Pure Liquids and Binary Liquid Mixtures. Landolt-Börnstein - Group IV Physical Chemistry (Numerical Data and Functional Relationships in Science and Technology), Vol 28; Lechner, M. D., Ed.; Springer: Berlin, 1997.

(70) Lee, O. S.; Liu, Y.; Schatz, G. C. Molecular Dynamics Simulation of $\beta$-Sheet Formation in SelfAssembled Peptide Amphiphile Fibers. J. Nanoparticle Res. 2012, 14 (8).

https://doi.org/10.1007/s11051-012-0936-z.

(71) Comer, J.; Gumbart, J. C.; Hénin, J.; Lelièvre, T.; Pohorille, A.; Chipot, C. The Adaptive Biasing Force Method: Everything You Always Wanted To Know but Were Afraid To Ask. J. Phys. Chem. B 2015, 119 (3), 1129-1151. https://doi.org/10.1021/jp506633n.

(72) Darve, E.; Rodríguez-Gómez, D.; Pohorille, A. Adaptive Biasing Force Method for Scalar and Vector Free Energy Calculations. J. Chem. Phys. 2008, 128 (14), 144120. https://doi.org/10.1063/1.2829861.

(73) Fiorin, G.; Klein, M. L.; Hénin, J. Using Collective Variables to Drive Molecular Dynamics Simulations. Mol. Phys. 2013, 111 (22-23), 3345-3362.

https://doi.org/10.1080/00268976.2013.813594.

(74) Wolfenden, R.; Andersson, L.; Cullis, P. M.; Southgate, C. C. B. Affinities of Amino Acid Side Chains for Solvent Water. Biochemistry 1981, 20 (4), 849-855.

https://doi.org/10.1021/bi00507a030.

(75) In, Y.; Chai, H. H.; No, K. T. A Partition Coefficient Calculation Method with the SFED Model. J. Chem. Inf. Model. 2005, 45 (2), 254-263. https://doi.org/10.1021/ci0498564.

(76) Gallicchio, E.; Zhang, L. Y.; Levy, R. M. The SGB/NP Hydration Free Energy Model Based on the Surface Generalized Born Solvent Reaction Field and Novel Nonpolar Hydration Free Energy Estimators. J. Comput. Chem. 2002, 23 (5), 517-529. https://doi.org/10.1002/jcc.10045.

(77) DeVane, R.; Shinoda, W.; Moore, P. B.; Klein, M. L. Transferable Coarse Grain Nonbonded Interaction Model for Amino Acids. J. Chem. Theory Comput. 2009, 5 (8), 2115-2124. https://doi.org/10.1021/ct800441u.

(78) Bulacu, M.; Goga, N.; Zhao, W.; Rossi, G.; Monticelli, L.; Periole, X.; Tieleman, D. P.; Marrink, S. J. Improved Angle Potentials for Coarse-Grained Molecular Dynamics Simulations. J. Chem. Theory Comput. 2013, 9 (8), 3282-3292. https://doi.org/10.1021/ct400219n. 


\section{Appendix A: Mapping Schemes}

Table A.1: Amino Acid Mapping Schemes

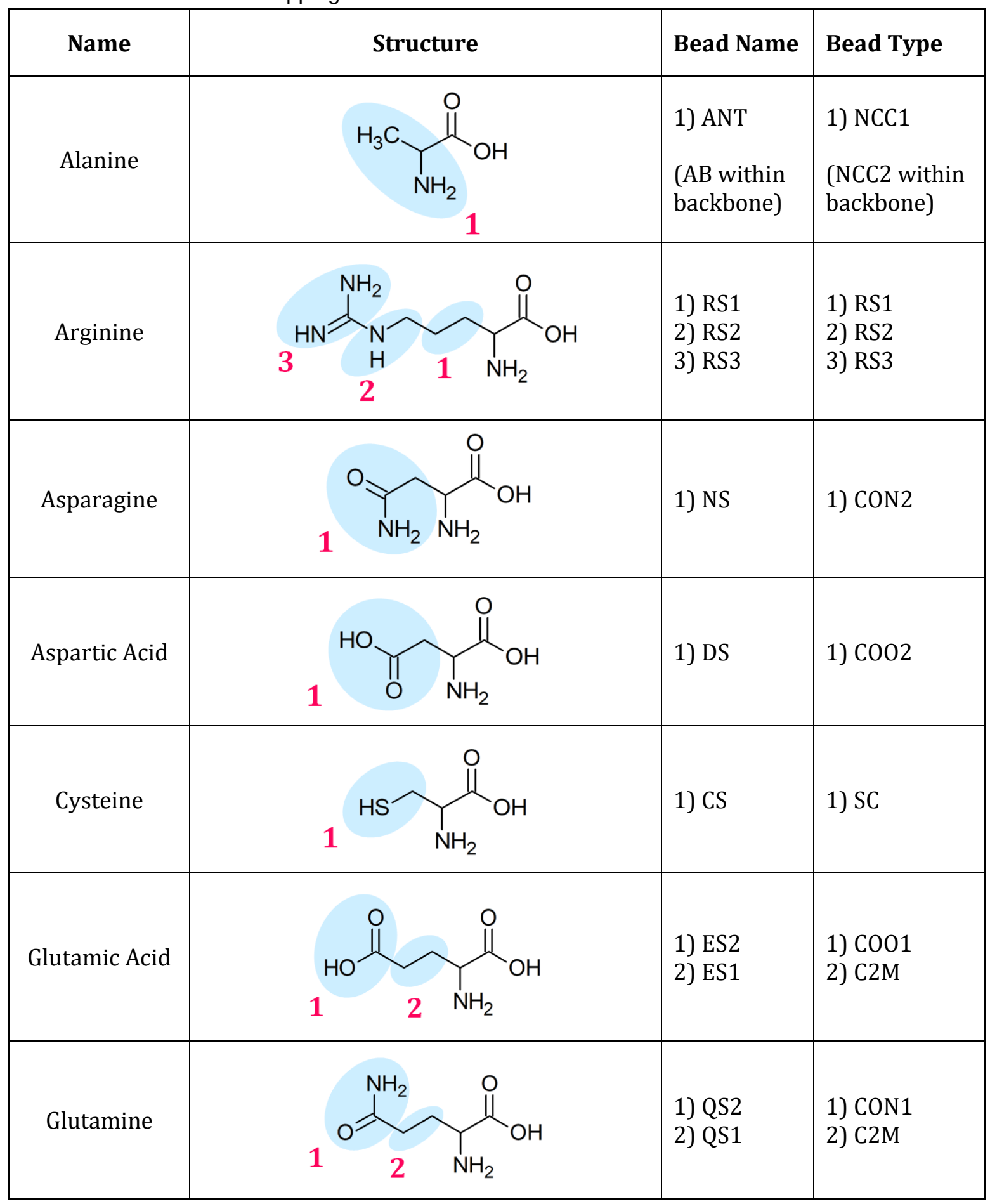


Table A.1, continued: Amino Acid Mapping Schemes

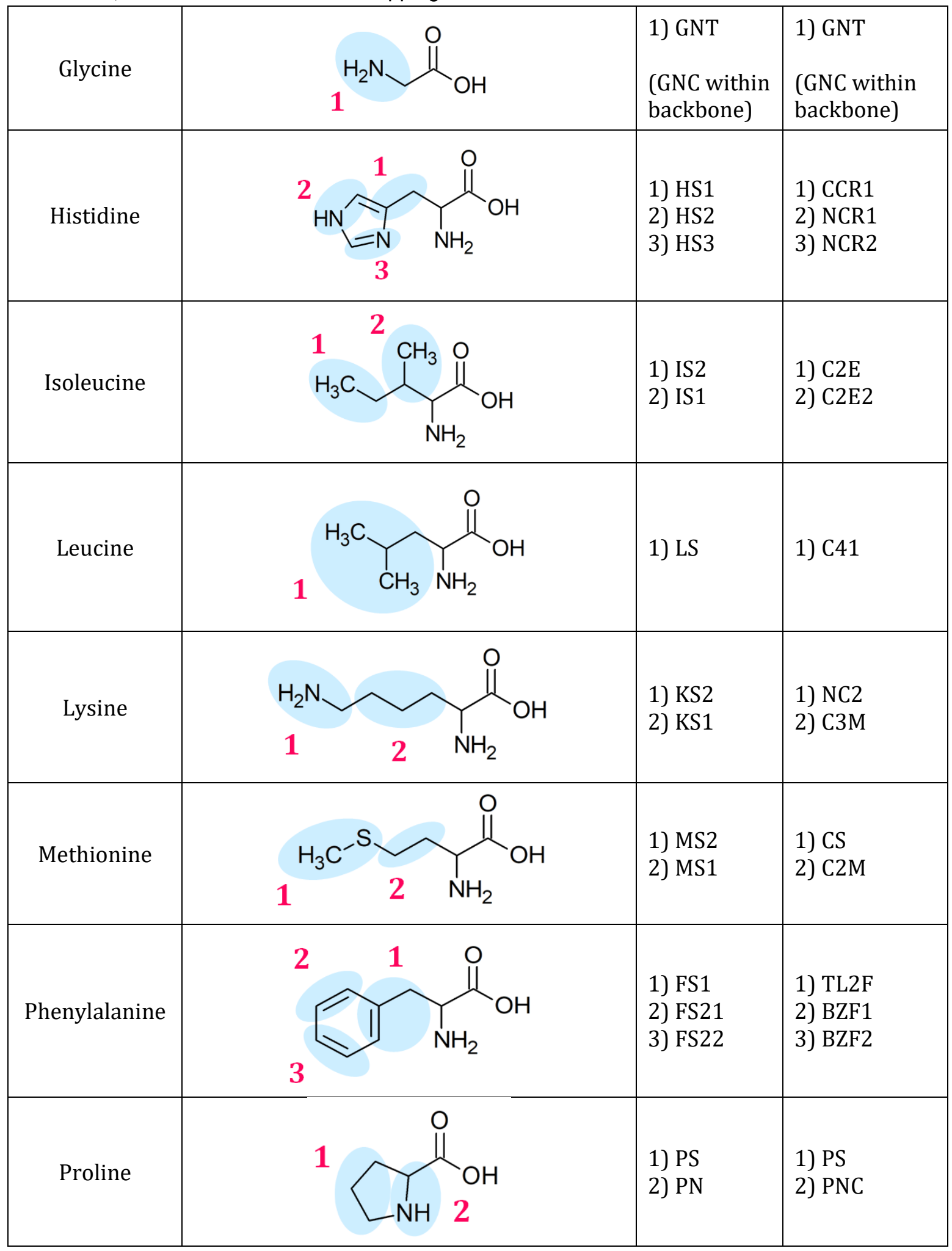


Table A.1, continued: Amino Acid Mapping Schemes

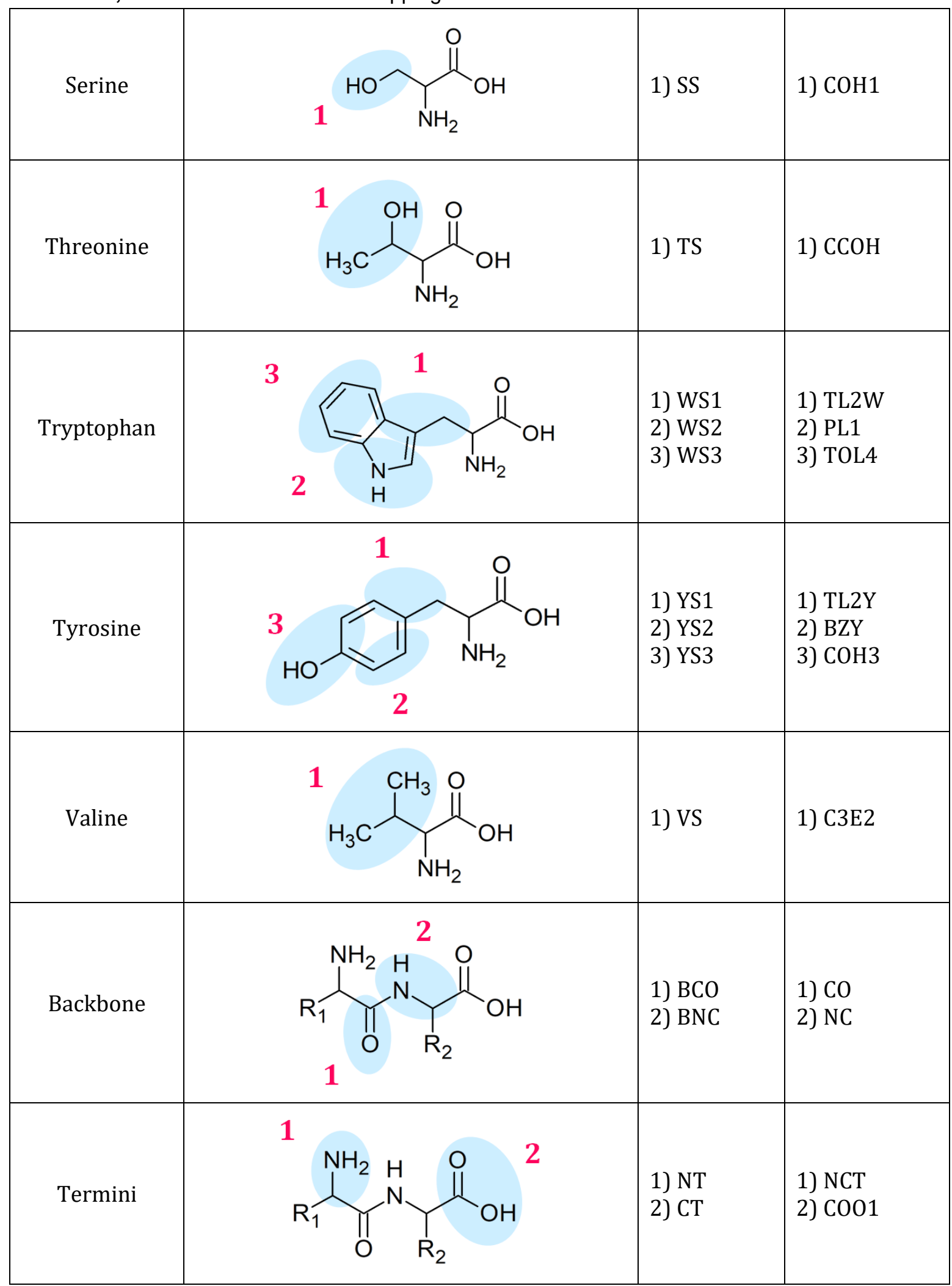


Table A.2: Analogue Mapping Schemes

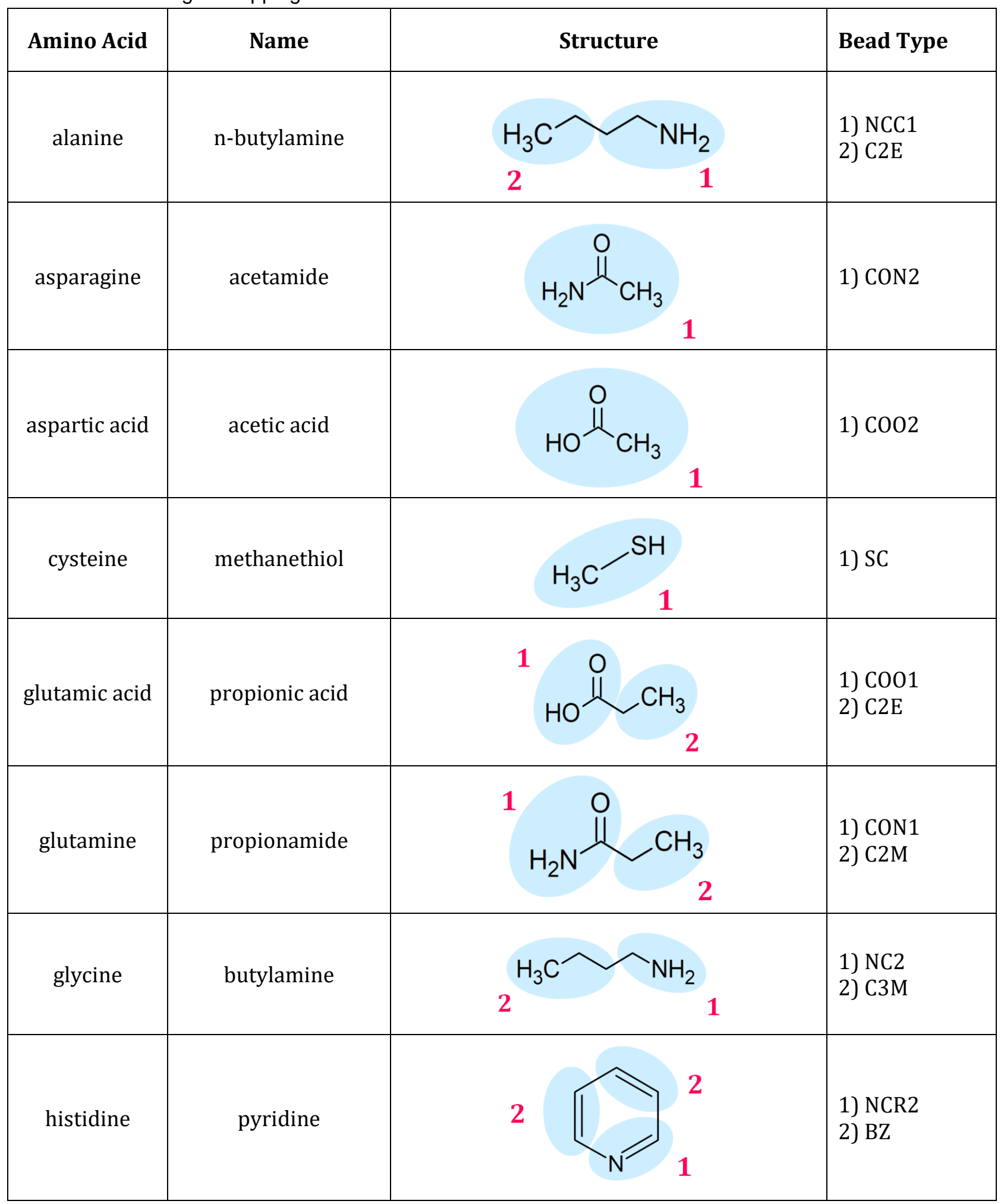


Table A.2, continued: Analogue Mapping Schemes

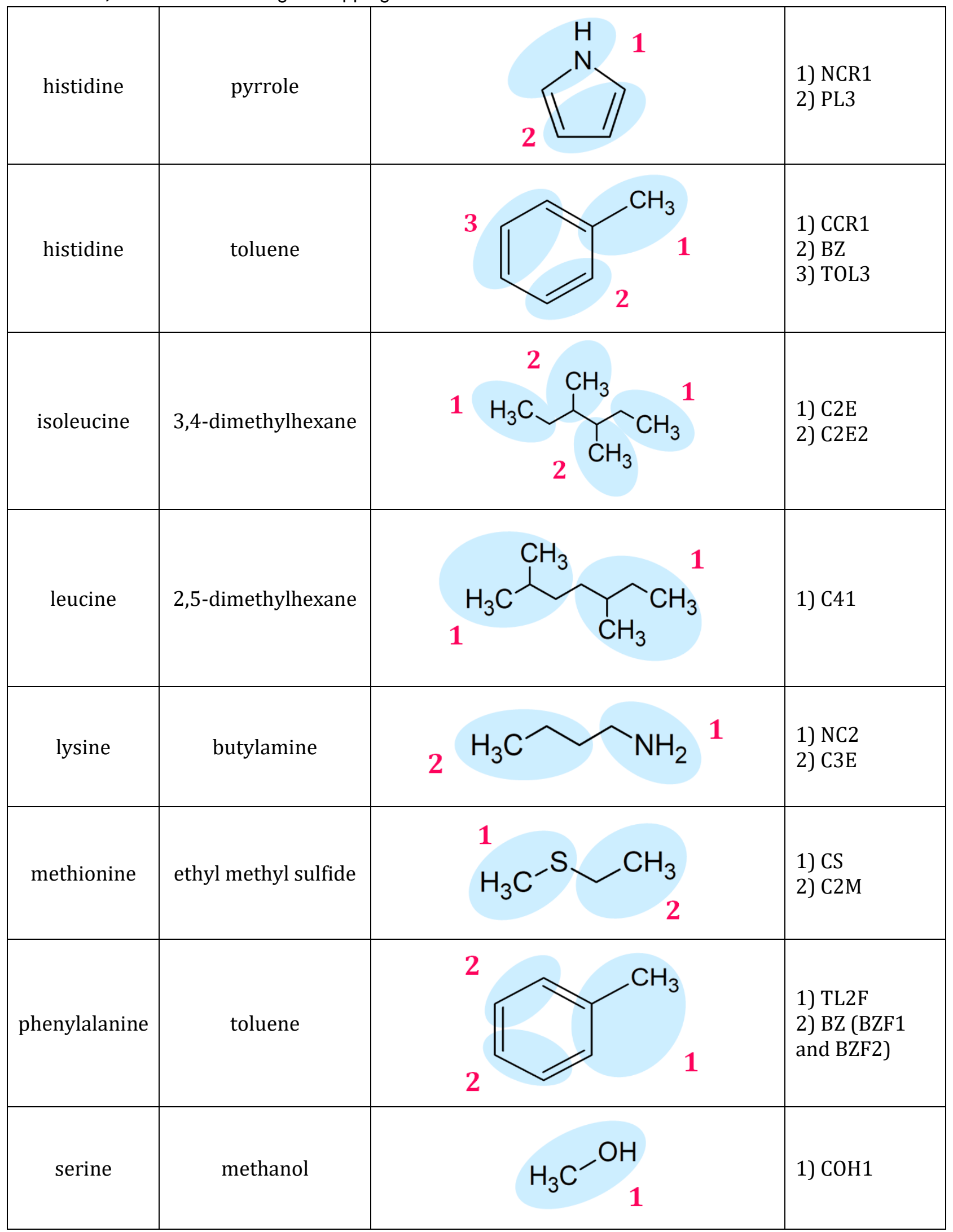


Table A.2, continued: Analogue Mapping Schemes

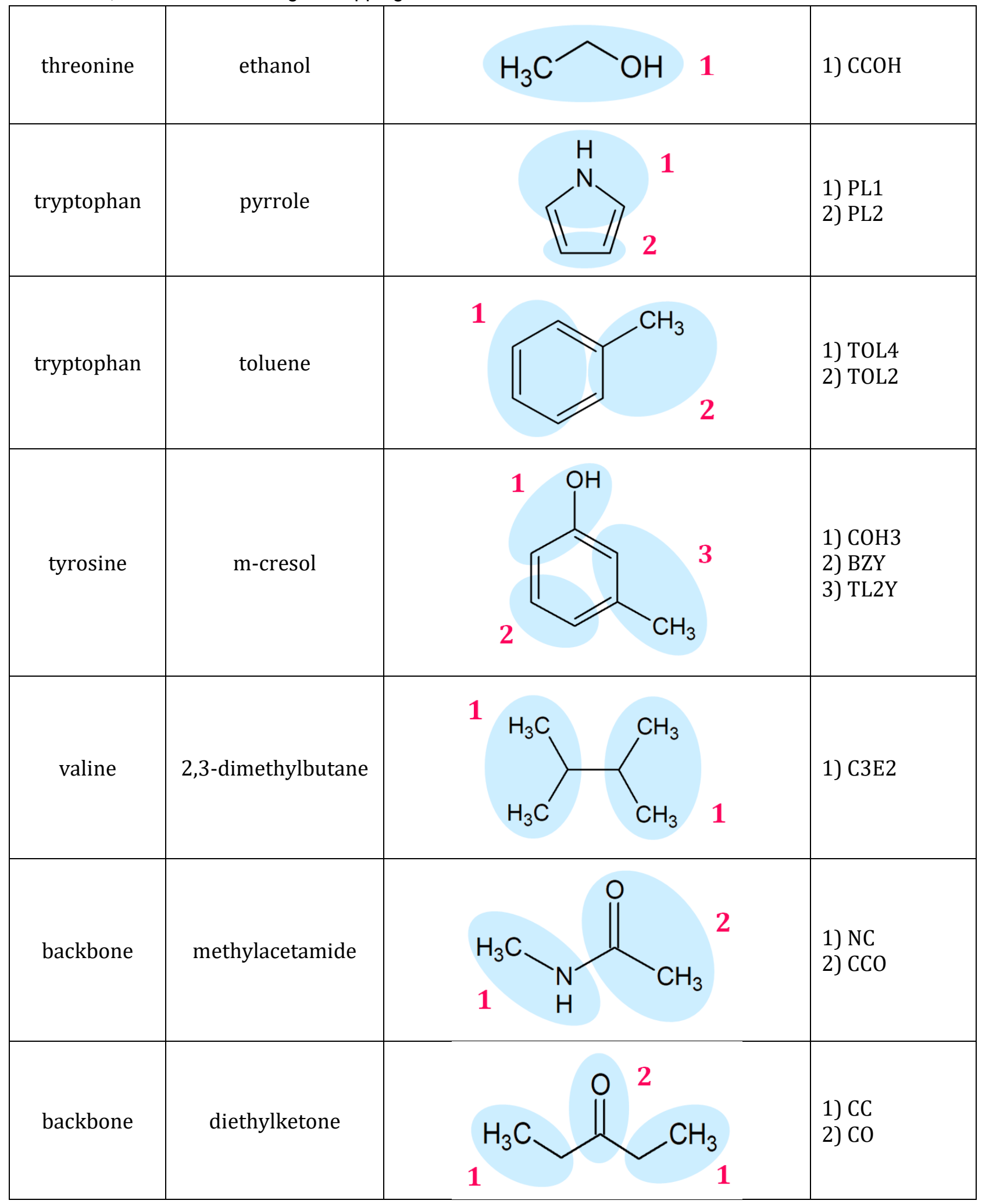


Table A.2, continued: Analogue Mapping Schemes

\begin{tabular}{|c|c|c|c|}
\hline $\mathrm{N}$-terminus & butylamine & $\widehat{\mathrm{NH}_{2}}$ & $\begin{array}{l}\text { 1) NCT } \\
\text { 2) C3E }\end{array}$ \\
\hline C-terminus & propionic acid & $1 \mathrm{CH}_{3}^{2}$ & $\begin{array}{l}\text { 1) } \mathrm{COO} 1 \\
\text { 2) } \mathrm{C} 2 \mathrm{E}\end{array}$ \\
\hline
\end{tabular}




\section{Appendix B: CG Analogue Parameter Development Results}

Table B.1: Comparison of experimental and CG model densities

\begin{tabular}{|c|c|c|c|c|c|}
\hline $\begin{array}{c}\text { Amino } \\
\text { Acid }\end{array}$ & Analogue & $\begin{array}{c}\text { Experimental } \\
\text { Density } \\
\left(\mathrm{g} / \mathrm{cm}^{3}\right)^{\mathrm{a}}\end{array}$ & $\begin{array}{c}\text { Model } \\
\text { Average } \\
\left(\mathrm{g} / \mathrm{cm}^{3}\right)\end{array}$ & \% Error & $\begin{array}{l}\text { Standard } \\
\text { Deviation }\end{array}$ \\
\hline Ala & N-butylamine & 0.741 & 0.728 & 1.78 & 7.93E-05 \\
\hline Asn & acetamide & 0.999 & 1.002 & 0.27 & $9.01 \mathrm{E}-05$ \\
\hline Asp & acetic acid & 1.043 & 1.042 & 0.11 & $1.20 \mathrm{E}-04$ \\
\hline Cys & methanethiol & 0.892 & 0.887 & 0.56 & $5.70 \mathrm{E}-05$ \\
\hline Gln & propionamide & 0.96 & 0.964 & 0.43 & $3.36 \mathrm{E}-05$ \\
\hline His & pyridine & 0.979 & 0.967 & 1.20 & $5.64 \mathrm{E}-05$ \\
\hline His & pyrrole & 0.965 & 0.944 & 2.22 & 7.97E-05 \\
\hline His & toluene & 0.865 & 0.856 & 1.07 & $5.94 \mathrm{E}-05$ \\
\hline Ile & 3,4-dimethylhexane & 0.716 & 0.703 & 1.81 & $4.02 \mathrm{E}-05$ \\
\hline Leu & 2,5-dimethylhexane & 0.694 & 0.687 & 0.96 & 4.49E-05 \\
\hline Lys & N-butylamine & 0.741 & 0.731 & 1.40 & $3.10 \mathrm{E}-05$ \\
\hline Met & ethyl methyl sulfide & 0.832 & 0.834 & 0.28 & $4.74 \mathrm{E}-05$ \\
\hline Phe & toluene & 0.865 & 0.862 & 0.40 & $3.36 \mathrm{E}-05$ \\
\hline Ser & methanol & 0.787 & 0.786 & 0.07 & $1.27 \mathrm{E}-04$ \\
\hline Thr & ethanol & 0.787 & 0.789 & 0.25 & $1.30 \mathrm{E}-04$ \\
\hline Trp & pyrrole & 0.965 & 0.957 & 0.83 & $3.86 \mathrm{E}-05$ \\
\hline Trp & toluene & 0.865 & 0.864 & 0.14 & 8.75E-05 \\
\hline Tyr & m-cresol & 1.03 & 1.036 & 0.53 & 5.59E-05 \\
\hline Val & 2,3-dimethylbutane & 0.658 & 0.652 & 0.85 & 4.17E-05 \\
\hline Backbone & N-methylacetamide & 0.9405 & 0.94152 & 0.11 & $5.73 \mathrm{E}-05$ \\
\hline Backbone & diethylketone & 0.8089 & 0.7843 & 3.04 & $6.50 \mathrm{E}-05$ \\
\hline
\end{tabular}

Table B.2: Comparison of experimental and CG model heats of vaporization

\begin{tabular}{|c|c|c|c|c|c|}
\hline $\begin{array}{c}\text { Amino } \\
\text { Acid }\end{array}$ & Analogue & $\begin{array}{c}\text { Experimental } \\
\text { Hvap } \\
\text { (kcal/mol)a }\end{array}$ & $\begin{array}{c}\text { Model } \\
\text { Average } \\
\text { (kcal/mol) }\end{array}$ & \% Error & $\begin{array}{c}\text { Standard } \\
\text { Deviation }\end{array}$ \\
\hline Ala & N-butylamine & 8.537 & 7.398 & 13.34 & $2.98 \mathrm{E}-03$ \\
\hline Asn & acetamide & $N A$ & -- & -- & -- \\
\hline Asp & acetic acid & 5.583 & 5.481 & 1.83 & $7.60 \mathrm{E}-04$ \\
\hline Cys & methanethiol & 5.872 & 5.544 & 5.59 & $4.75 \mathrm{E}-04$ \\
\hline Gln & propionamide & $N A$ & -- & -- & -- \\
\hline His & pyridine & 9.61 & 8.72 & 9.28 & $4.58 \mathrm{E}-03$ \\
\hline His & pyrrole & 10.78 & 8.89 & 17.55 & $2.95 \mathrm{E}-03$ \\
\hline
\end{tabular}


Table B.2, continued: Comparison of experimental and CG model heats of vaporization

\begin{tabular}{|c|c|c|c|c|c|}
\hline His & toluene & 9.0846 & 8.5631 & 5.74 & $5.84 \mathrm{E}-03$ \\
\hline Ile & 3,4-dimethylhexane & 9.314 & 9.570 & 2.75 & $6.41 \mathrm{E}-03$ \\
\hline Leu & 2,5-dimethylhexane & 9.046 & 8.398 & 7.16 & $2.41 \mathrm{E}-03$ \\
\hline Lys & N-butylamine & 8.537 & 7.092 & 16.93 & $3.33 \mathrm{E}-03$ \\
\hline Met & ethyl methyl sulfide & 7.6123 & 7.0342 & 7.59 & $1.46 \mathrm{E}-03$ \\
\hline Phe & toluene & 9.0846 & 8.4578 & 6.90 & $4.59 \mathrm{E}-03$ \\
\hline Ser & methanol & 8.946 & 4.312 & 51.80 & $4.68 \mathrm{E}-04$ \\
\hline Thr & ethanol & 10.115 & 4.900 & 51.56 & $9.41 \mathrm{E}-04$ \\
\hline Trp & pyrrole & 10.78 & 9.31 & 13.60 & $1.95 \mathrm{E}-03$ \\
\hline Trp & toluene & 9.0846 & 7.9952 & 11.99 & $1.77 \mathrm{E}-03$ \\
\hline Tyr & m-cresol & 14.749 & 10.417 & 29.37 & $3.13 \mathrm{E}-03$ \\
\hline Val & 2,3-dimethylbutane & 6.96 & 6.47 & 7.05 & $2.04 \mathrm{E}-03$ \\
\hline Backbone & N-methylacetamide & $N A$ & -- & -- & -- \\
\hline Backbone & diethylketone & 9.2065 & 8.4050 & 8.71 & $4.73 \mathrm{E}-03$ \\
\hline a Experimental data from refs 63,65,68 & & & \\
\hline
\end{tabular}

Table B.3: Comparison of experimental and CG model surface tensions

\begin{tabular}{|c|c|c|c|c|c|}
\hline $\begin{array}{c}\text { Amino } \\
\text { Acid }\end{array}$ & Analogue & $\begin{array}{c}\text { Experimental } \\
\text { Surface } \\
\text { Tension } \\
\text { (mN/m)a }\end{array}$ & $\begin{array}{c}\text { Model } \\
\text { Average } \\
\mathbf{( m N / m )}\end{array}$ & \% Error & $\begin{array}{c}\text { Standard } \\
\text { Deviation }\end{array}$ \\
\hline Ala & N-butylamine & 23.44 & 23.787 & 1.48 & 0.159 \\
\hline Asn & acetamide & 38.96 & 38.963 & 0.01 & 0.157 \\
\hline Asp & acetic acid & 27.1 & 28.172 & 3.96 & 0.223 \\
\hline Cys & methanethiol & 31 & 31.510 & 1.65 & 0.133 \\
\hline Gln & propionamide & 31.31 & 31.259 & 0.16 & 0.150 \\
\hline His & pyridine & 36.72 & 37.502 & 2.13 & 0.223 \\
\hline His & pyrrole & 36.32 & 38.040 & 4.74 & 0.143 \\
\hline His & toluene & 27.93 & 28.043 & 0.40 & 0.064 \\
\hline Ile & 3,4 -dimethylhexane & 21.21 & 22.578 & 6.45 & 0.104 \\
\hline Leu & 2,5 -dimethylhexane & 19.28 & 20.420 & 5.91 & 0.220 \\
\hline Lys & N-butylamine & 23.44 & 23.720 & 1.20 & 0.092 \\
\hline Met & ethyl methyl sulfide & 24.42 & 25.651 & 5.04 & 0.114 \\
\hline Phe & toluene & 27.93 & 29.197 & 4.54 & 0.177 \\
\hline Ser & methanol & 22.07 & 22.655 & 2.65 & 0.121 \\
\hline Thr & ethanol & 21.97 & 22.833 & 3.93 & 0.037 \\
\hline Trp & pyrrole & 36.32 & 35.902 & 1.15 & 0.147 \\
\hline Trp & toluene & 27.93 & 28.335 & 1.45 & 0.062 \\
\hline Tyr & m-cresol & 35.76 & 37.381 & 4.53 & 0.226 \\
\hline Val & 2,3 -dimethylbutane & 16.87 & 17.073 & 1.20 & 0.113 \\
\hline
\end{tabular}


Table B.3, continued: Comparison of experimental and CG model surface tensions

\begin{tabular}{|c|c|c|c|c|c|}
\hline Backbone & N-methylacetamide & 32.53 & 32.933 & 1.24 & 0.160 \\
\hline Backbone & diethylketone & 24.74 & 26.565 & 7.38 & 0.160 \\
\hline
\end{tabular}




\section{Appendix C: Peptides and Analogues, Distributions and RDFs}

\section{C1: Alanine}

Ala-Ala-Ala Tripeptide

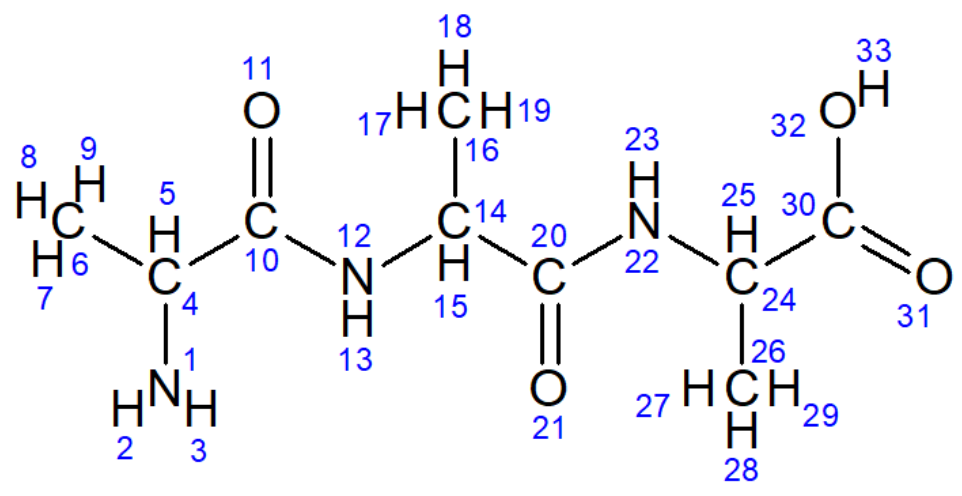

Figure C1.1: All-atom Alanine tripeptide with atom numbering

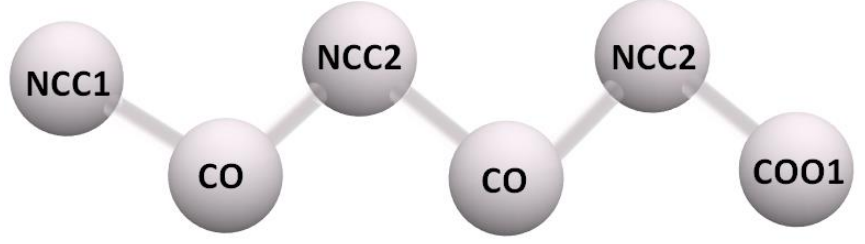

Figure C1.2: CG model of Alanine tripeptide showing bead types
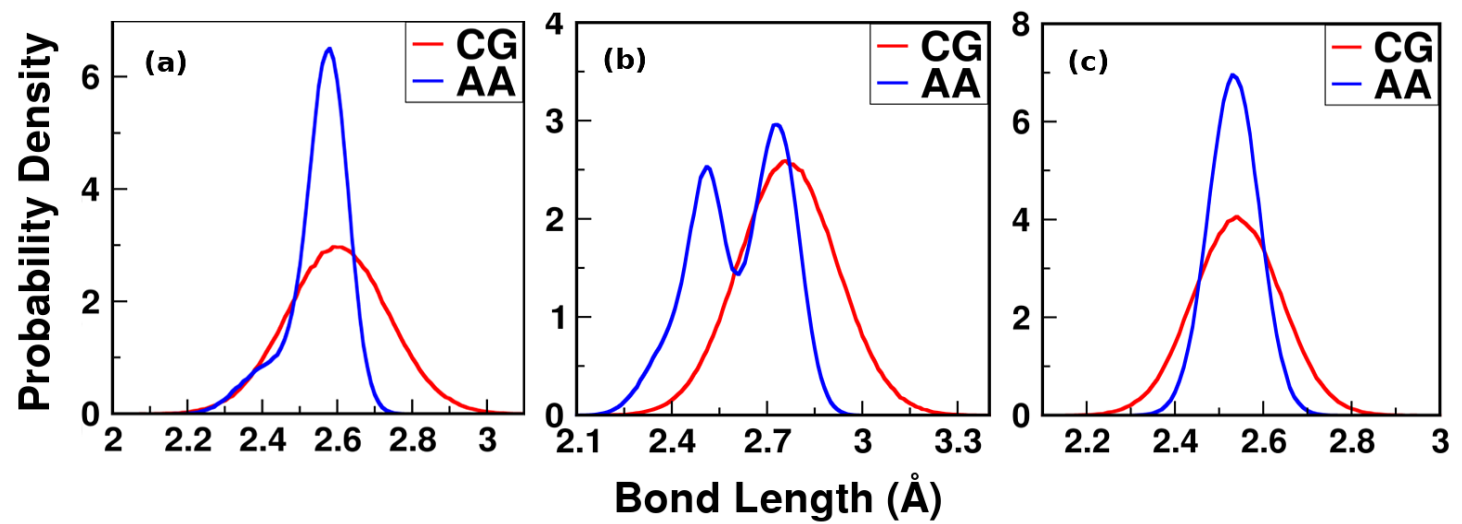

Figure C1.3: Distributions of the bonds (a) NCC1-CO, (b) CO-NCC2, and (c) NCC2-COO1 


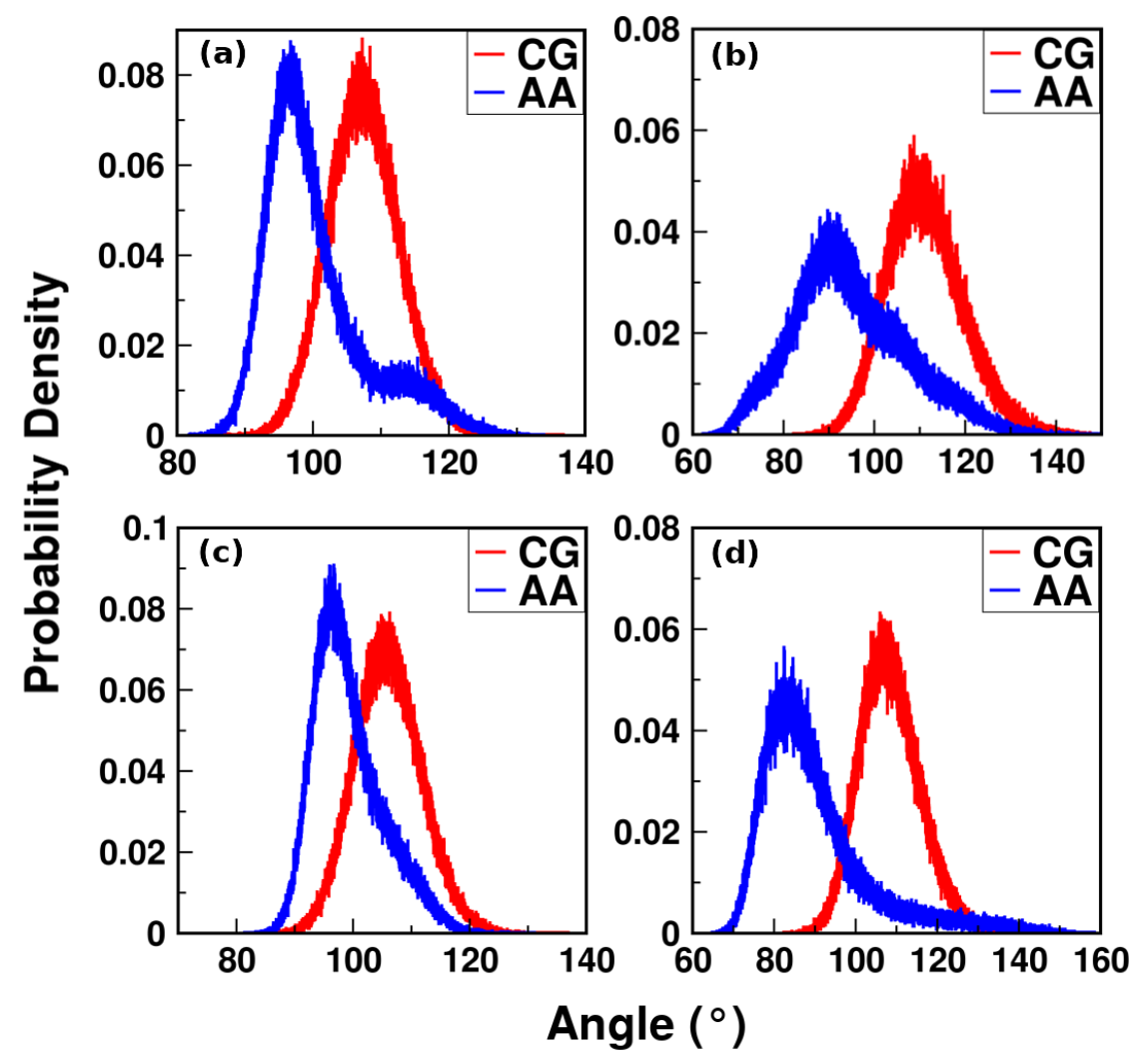

Figure C1.4: Distributions of angles (a) NCC1-CO-NCC2, (b) CO-NCC2-CO, (c) NCC2-CO-NCC2, and (d) CO-NCC2-COO1
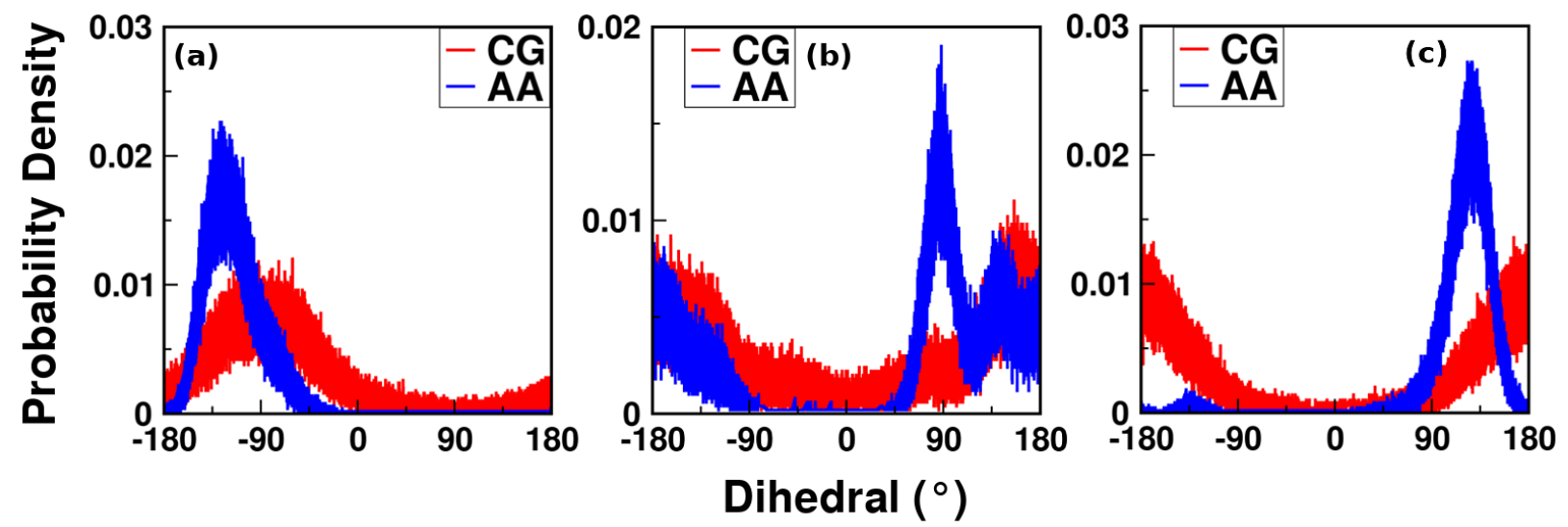

Figure C1.5: Distributions of dihedrals (a) NCC1-CO-NCC2-CO (b) CO-NCC2-CO-NCC2, and (c) NCC2CO-NCC2-COO1 


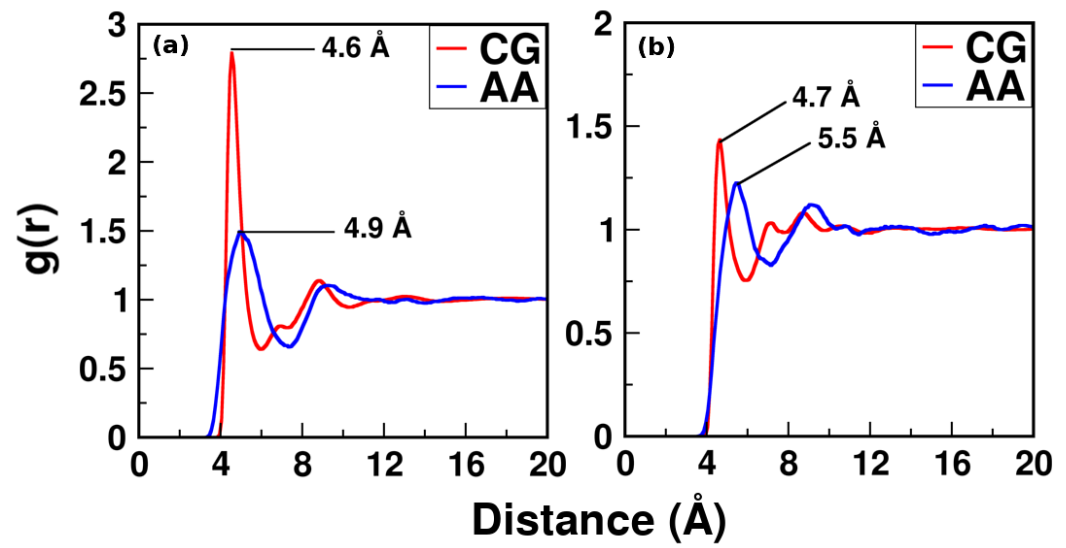

Figure C1.6: RDFs of (a) NCC1 and (b) NCC2

\section{Analogue: N-butylamine}

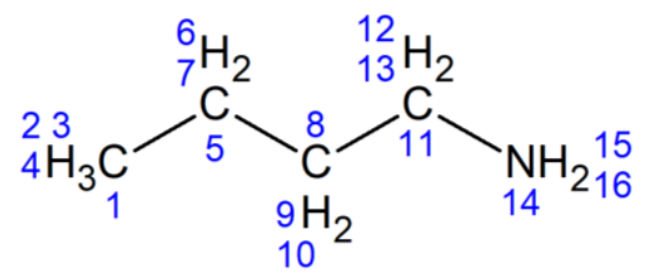

Figure C1.7: All-atom N-butylamine with atom numbering

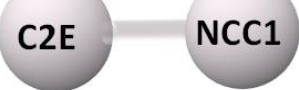

Figure C1.8: CG model of N-butylamine showing bead types

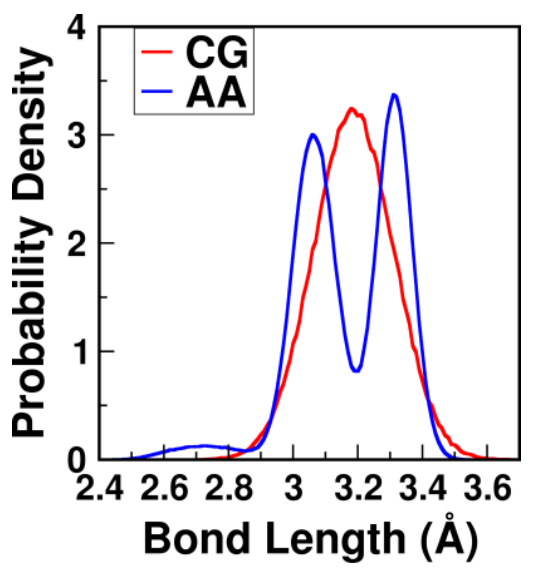

Figure C1.9: Distribution of bond NCC1-C2E 


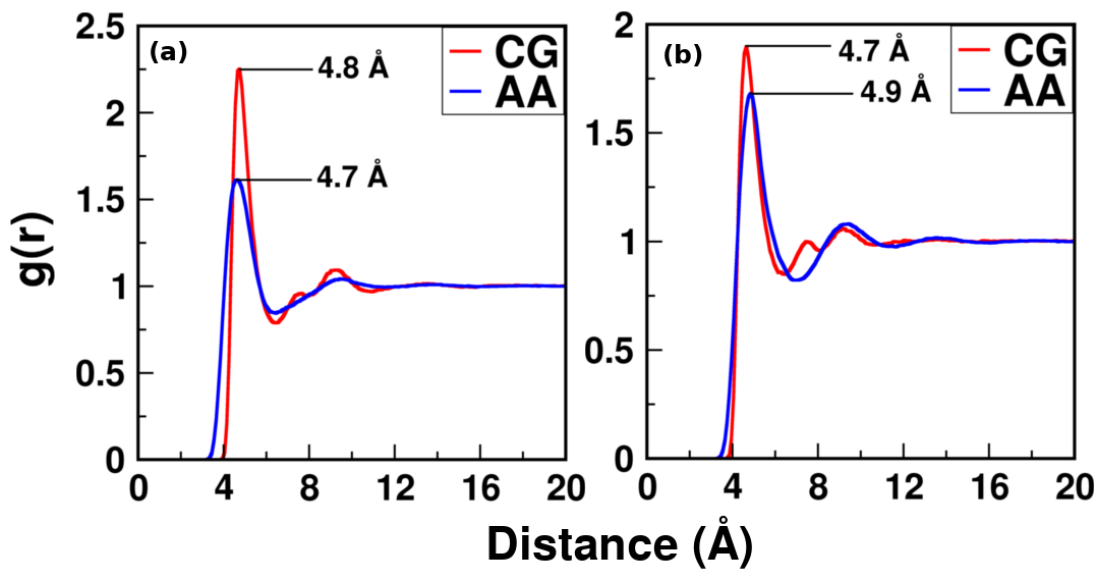

Figure C1.10: RDFs of (a) NCC1 and (b) C2E 


\section{C2: Arginine}

Table C2.1: Arginine side chain atom charges.

Hydrogens HB3 and HH22 are not in the neutral side chain; NA signifies "Not Available" in consequence.

Atom names are CHARMM atom names.

\begin{tabular}{|c|c|c|c|}
\hline & \multicolumn{3}{|c|}{ Charges } \\
\hline $\begin{array}{c}\text { Atom } \\
\text { Name }\end{array}$ & CHARMM & Gaussian & Assigned \\
\hline CB & -0.18 & -0.518 & -0.380 \\
\hline HB1 & 0.09 & 0.137 & 0.137 \\
\hline HB2 & 0.09 & 0.139 & 0.137 \\
\hline HB3 & NA & $\mathbf{0 . 1 3 6}$ & $\boldsymbol{N A}$ \\
\hline CG & -0.18 & -0.091 & -0.091 \\
\hline HG1 & 0.09 & 0.143 & 0.130 \\
\hline HG2 & 0.09 & 0.116 & 0.130 \\
\hline CD & 0.20 & -0.317 & -0.317 \\
\hline HD1 & 0.09 & 0.162 & 0.151 \\
\hline HD2 & 0.09 & 0.140 & 0.151 \\
\hline NE & -0.70 & -0.177 & -0.177 \\
\hline HE & 0.44 & 0.221 & 0.221 \\
\hline CZ & 0.64 & 0.099 & 0.099 \\
\hline NH1 & -0.80 & -0.445 & -0.445 \\
\hline HH11 & 0.46 & 0.186 & 0.186 \\
\hline HH22 & $\mathbf{0 . 4 6}$ & $\boldsymbol{N A}$ & $\boldsymbol{N A}$ \\
\hline NH2 & -0.80 & -0.380 & -0.380 \\
\hline HH21 & 0.46 & 0.228 & 0.224 \\
\hline HH22 & 0.46 & 0.220 & 0.224 \\
\hline Total & $\mathbf{1}$ & $-\mathbf{0 . 0 0 1}$ & $\mathbf{0}$ \\
\hline
\end{tabular}




\section{Ala-Arg Dipeptide}

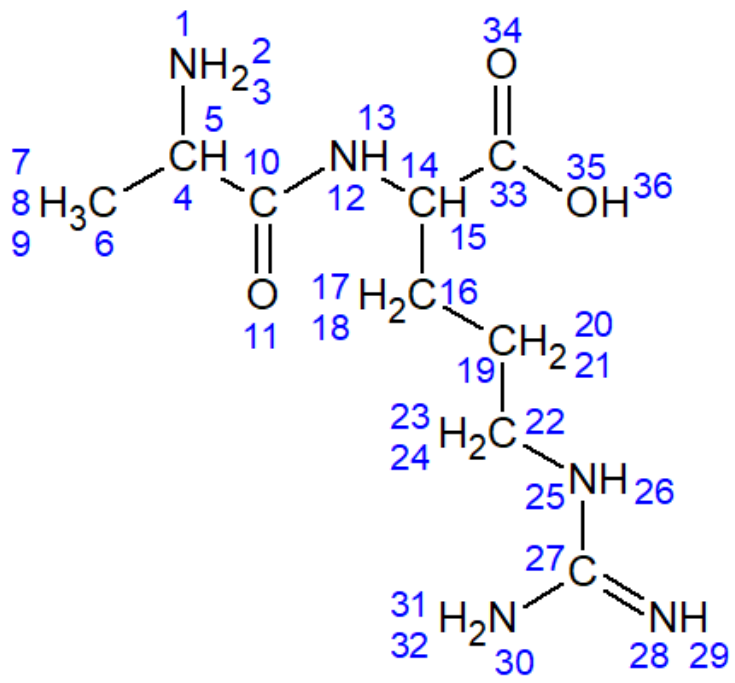

Figure C2.1: All-atom Alanyl-Arginine dipeptide with atom numbering

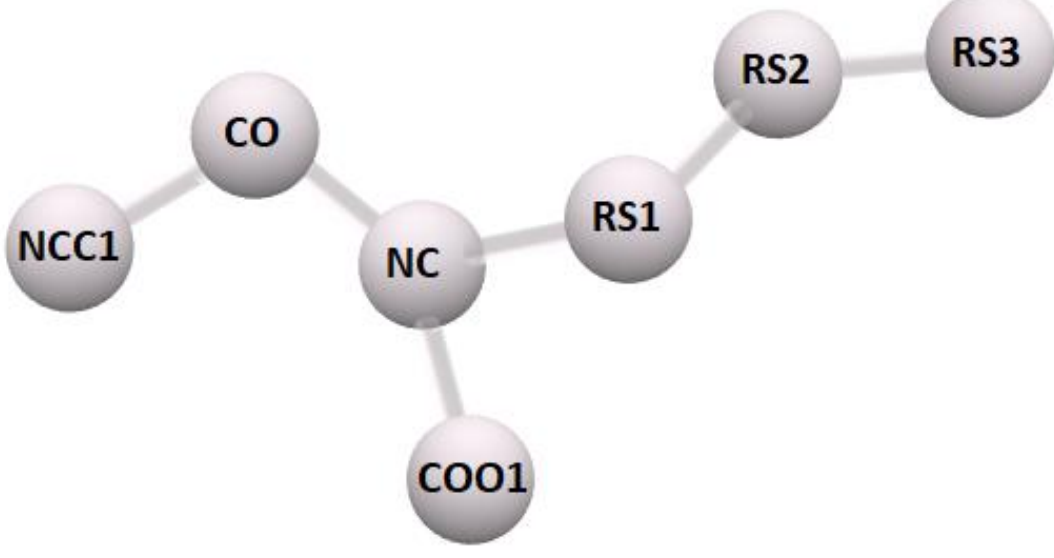

Figure C2.2: CG model of Alanyl-Arginine dipeptide showing bead types 

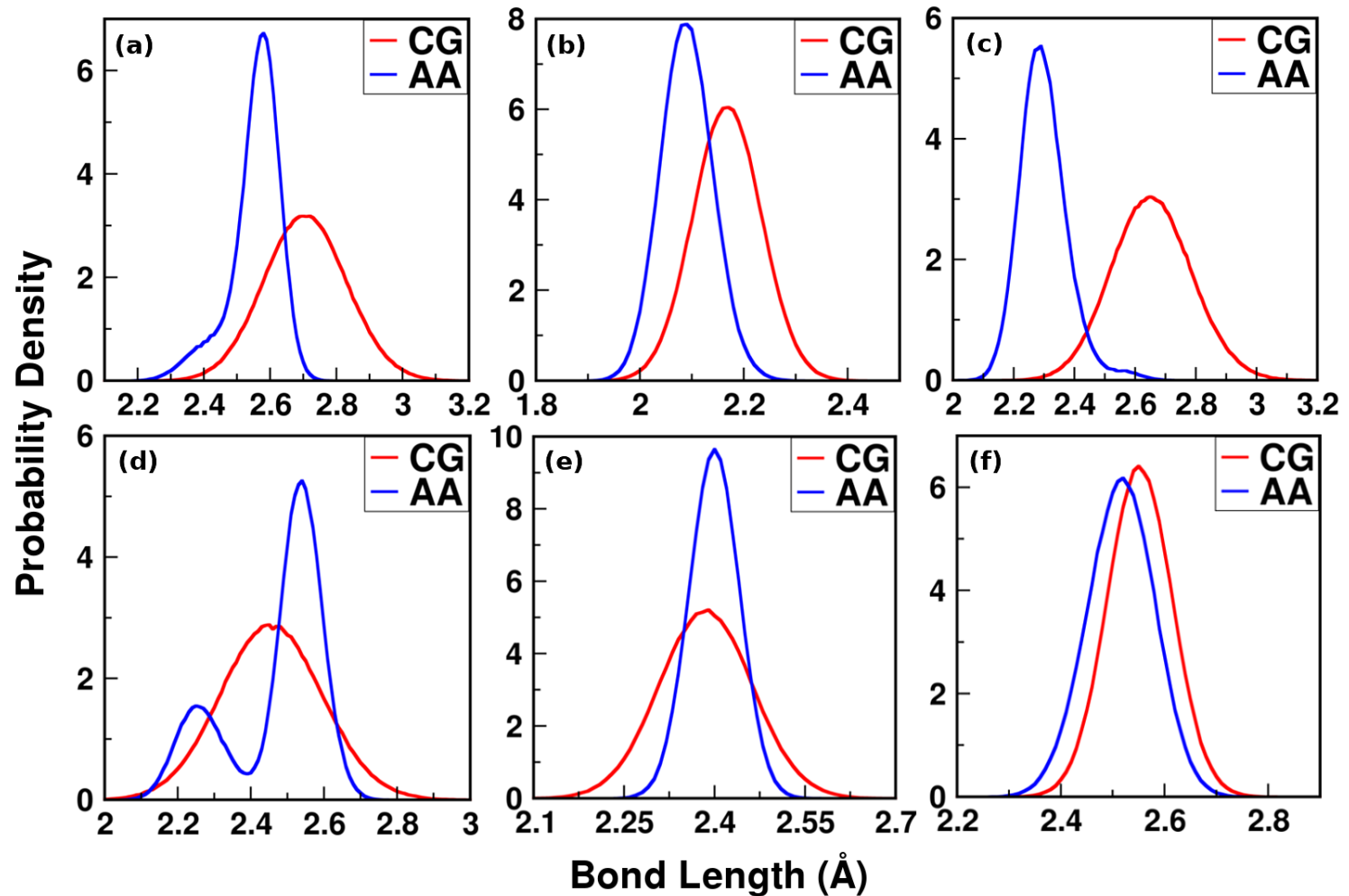

Figure C2.3: Distribution of bonds (a) NCC1-CO, (b) CO-NC, (c) NC-RS1, (d) RS1-RS2, (e) RS2-RS3, and (f) NC-COO1
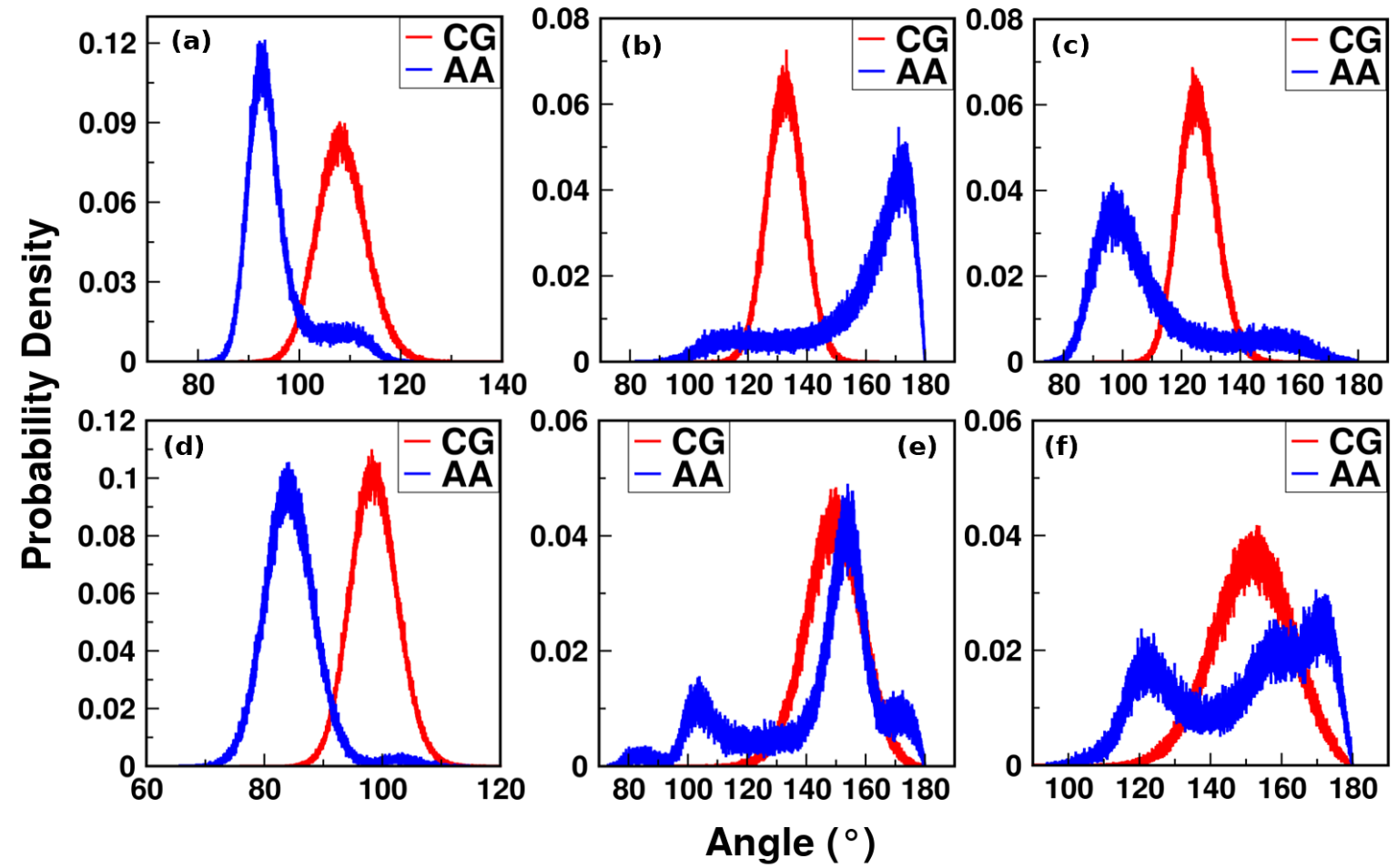

Figure C2.4: Distributions of angles (a) NCC1-CO-NC, (b) CO-NC-RS1, (c) CO-NC-COO1, (d) RS1-NCCOO1, (e) NC-RS1-RS2, and (f) RS1-RS2-RS3 

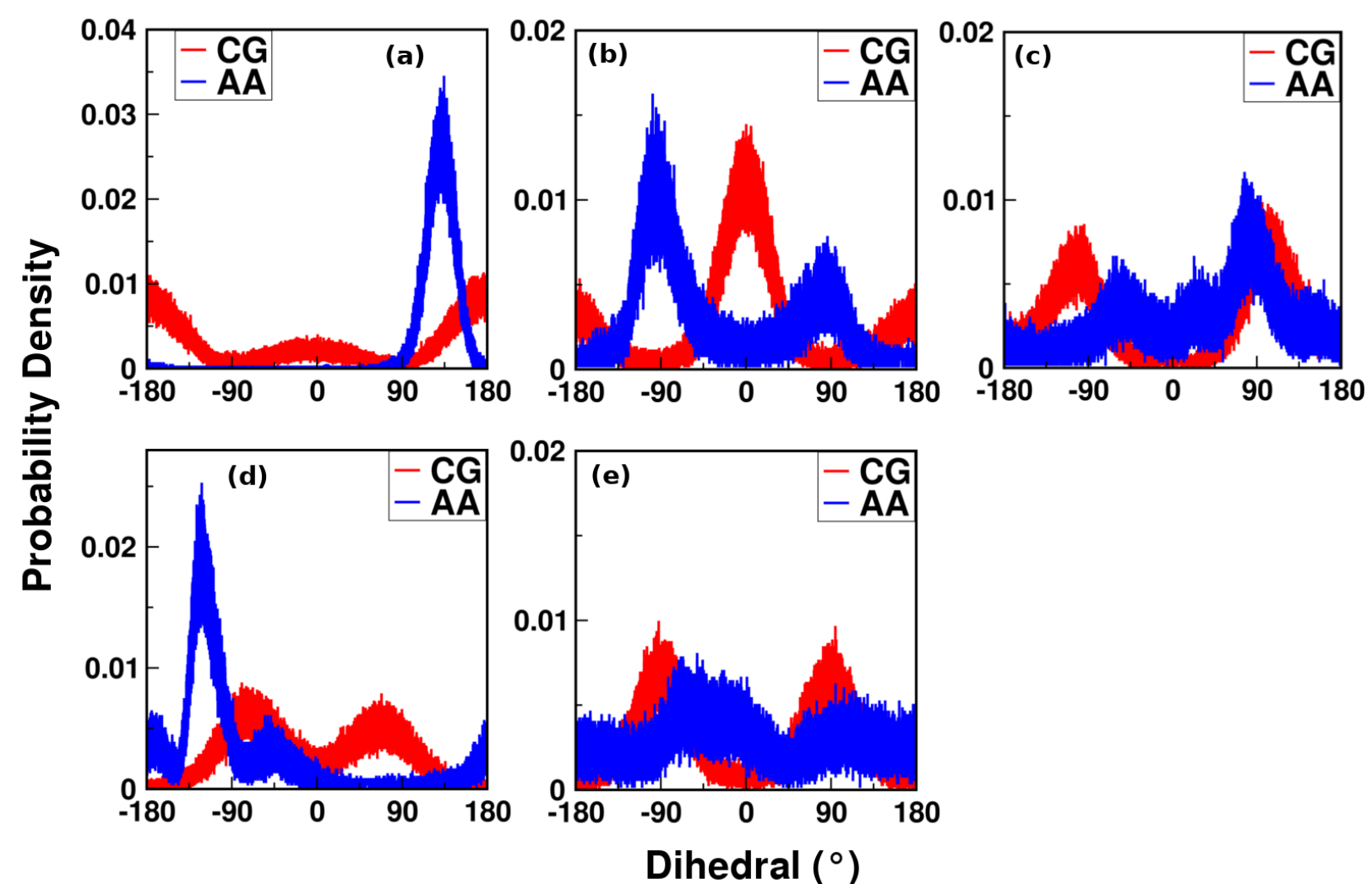

Figure C2.5: Distributions of dihedrals (a) NCC1-CO-NC-COO1, (b) NCC1-CO-NC-RS1, (c) CO-NCRS1-RS2 (d) COO1-NC-RS1-RS2, and (e) NC-RS1-RS2-RS3
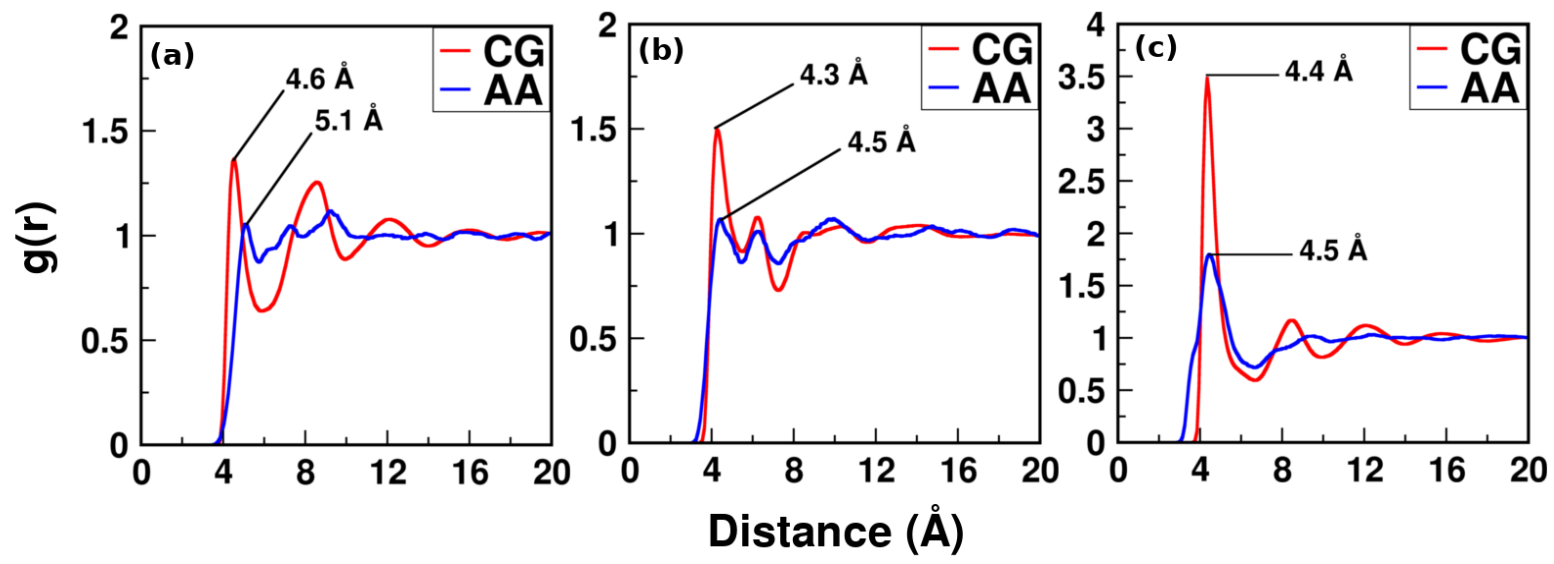

Figure C2.6: RDFs of (a) RS1, (b) RS2, and (c) RS3 


\section{C3: Asparagine}

Asn-Asn Dipeptide

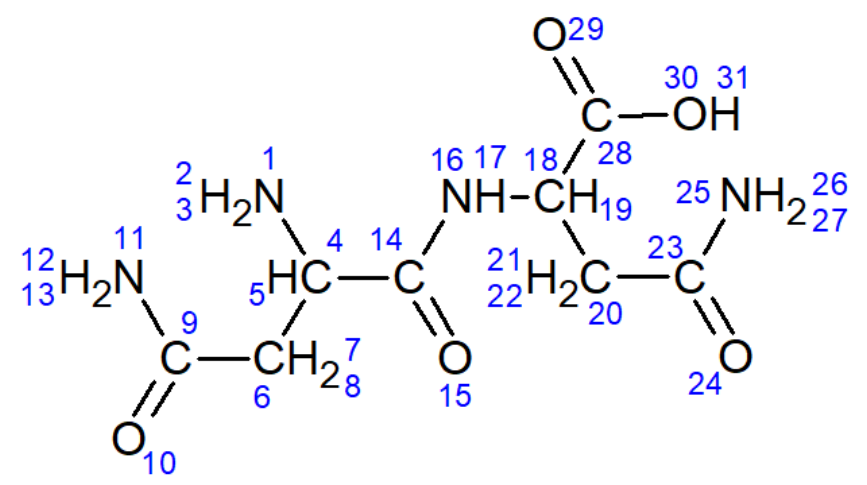

Figure C3.1: All-atom Asparagine dipeptide with atom numbering

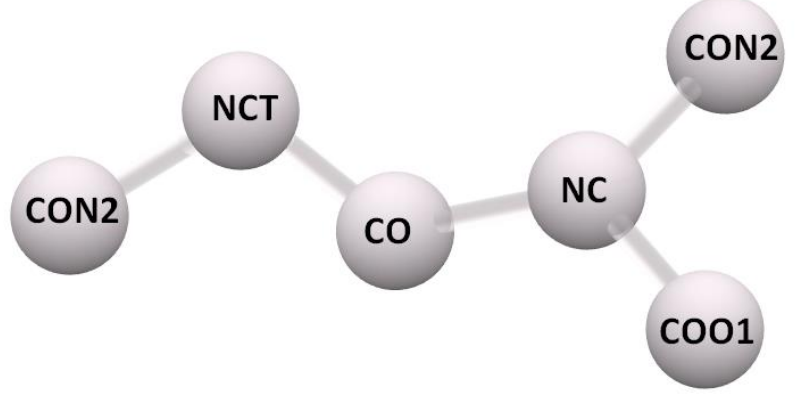

Figure C3.2: CG model of Asparagine dipeptide showing bead types 

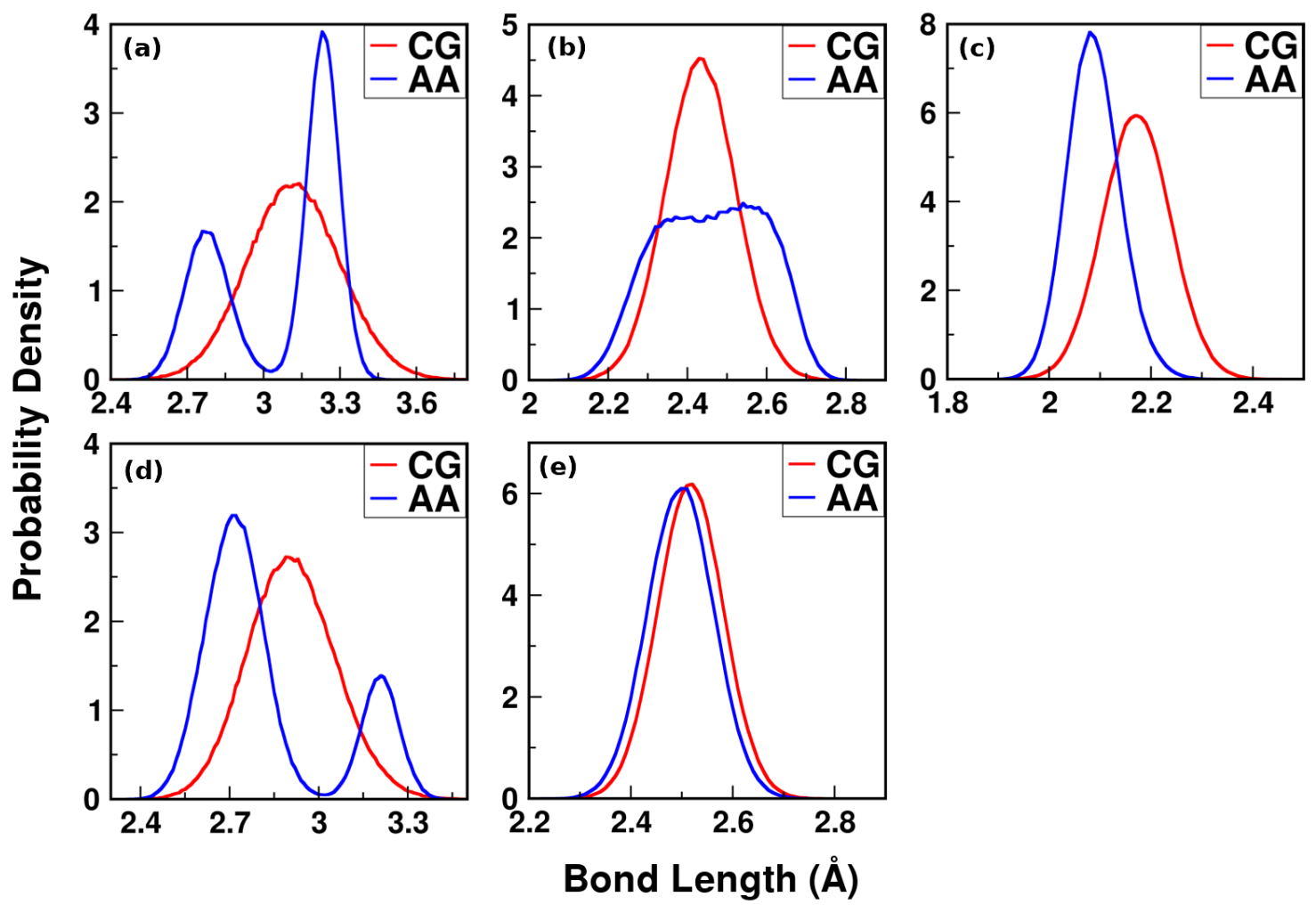

Figure C3.3: Distributions of bonds (a) NS-NCT, (b) NCT-CO, (c) CO-NC, (d) NC-CON2, and (e) NCCOO1
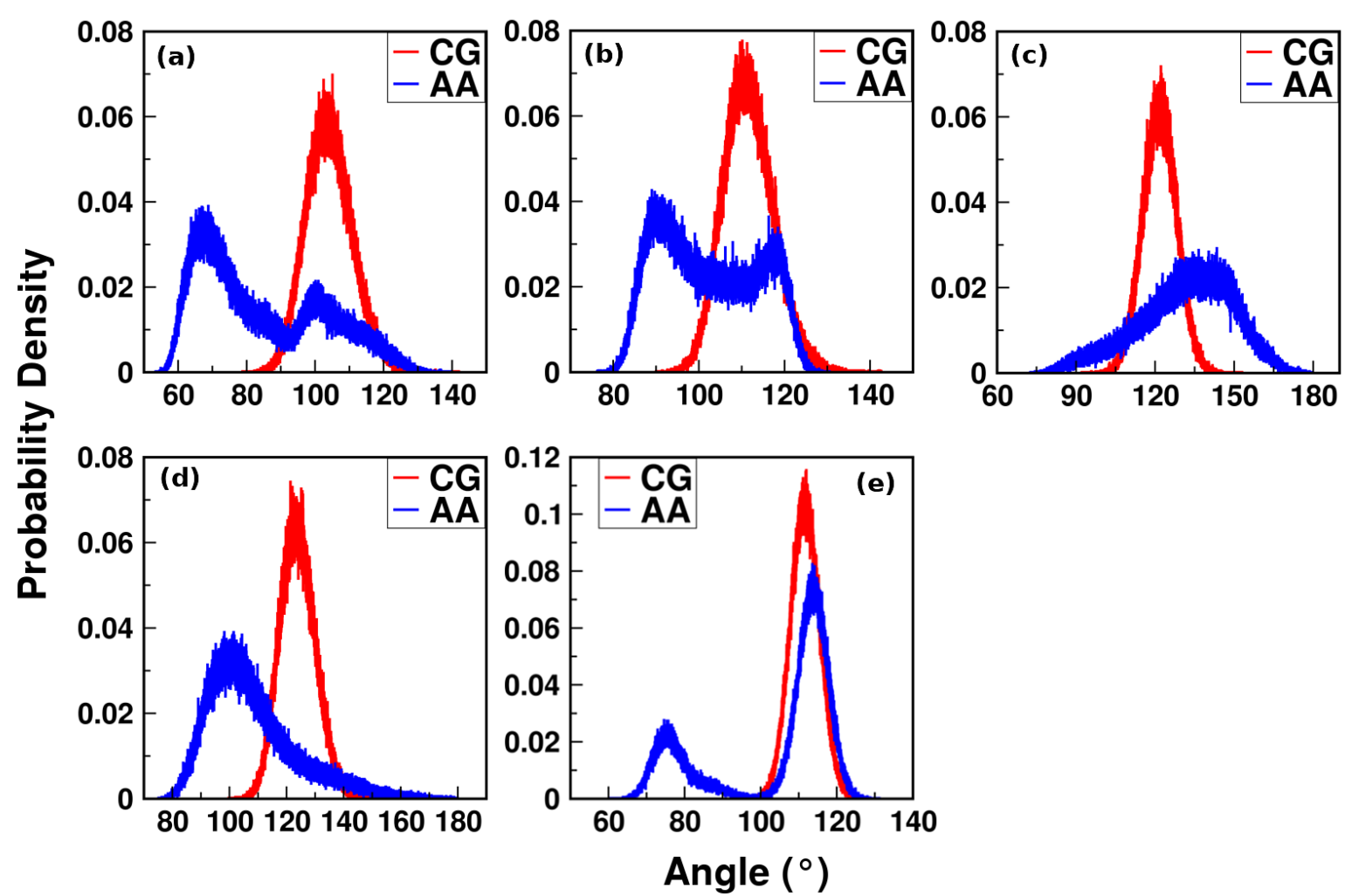

Figure C3.4: Distributions of angles (a) CON2-NT-CO, (b) NT-CO-NC, (c) CO-NC-CON2, (d) CO-NCCOO1, and (e) CON2-NC-COO1 

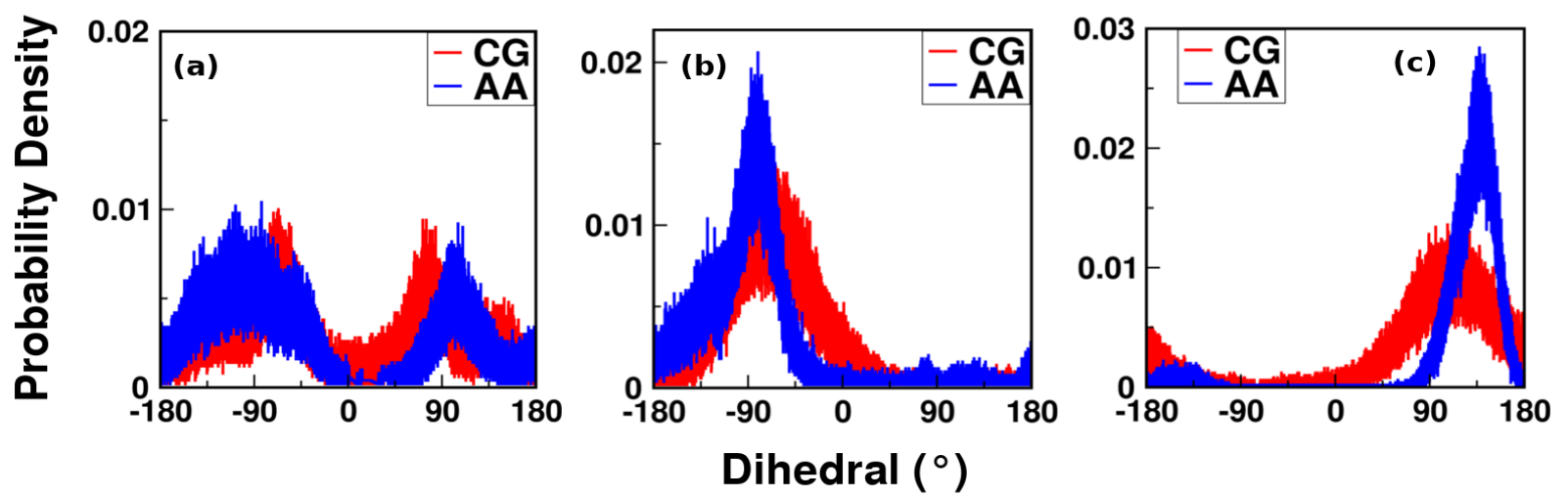

Figure C3.5: Distributions of dihedrals (a) CON2-NCT-CO-NC, (b) NCT-CO-NC-CON2, and (c) NCT-CONC-COO1

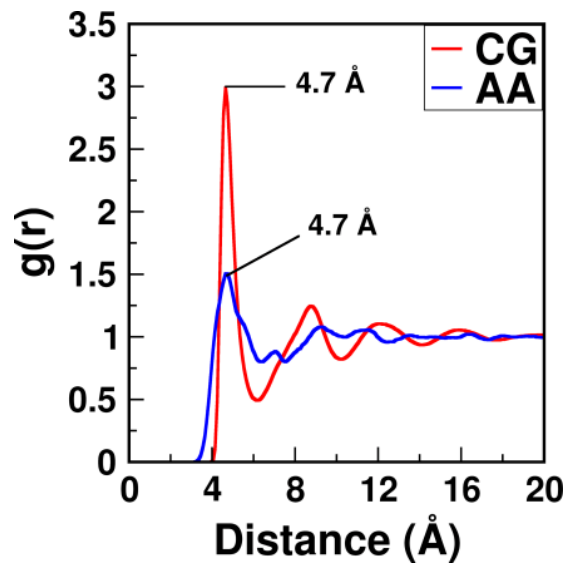

Figure C3.6: RDF of CON2

Analogue: Acetamide<smiles>CC(N)=O</smiles>

Figure C3.7: All-atom acetamide

\section{CON2}

Figure C3.8: CG model of acetamide showing bead type 


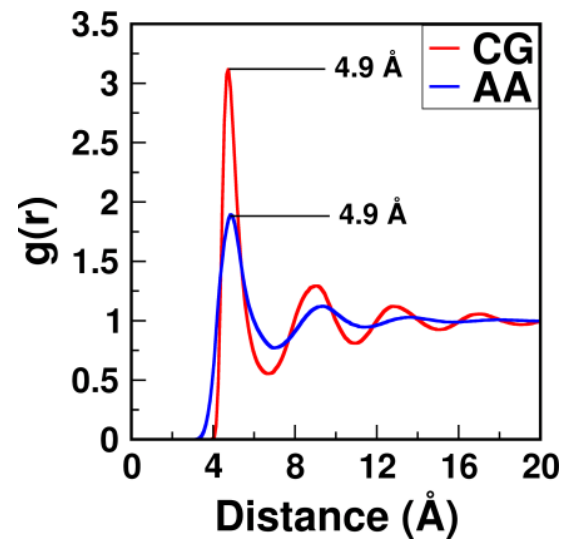

Figure C3.9: RDF of CON2 


\section{C4: Aspartic Acid}

\section{Asp-Asp Dipeptide}

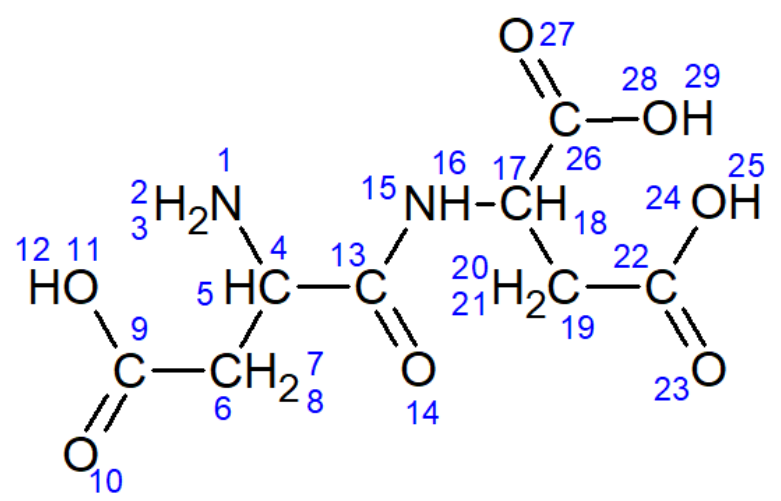

Figure C4.1: All-atom Aspartic Acid dipeptide with atom numbering

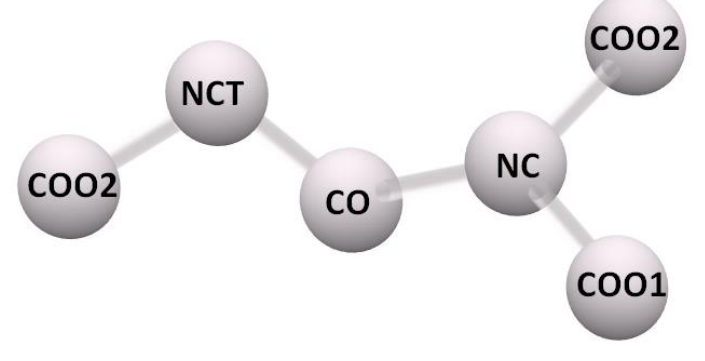

Figure C4.2: CG model of Aspartic Acid dipeptide showing bead types 

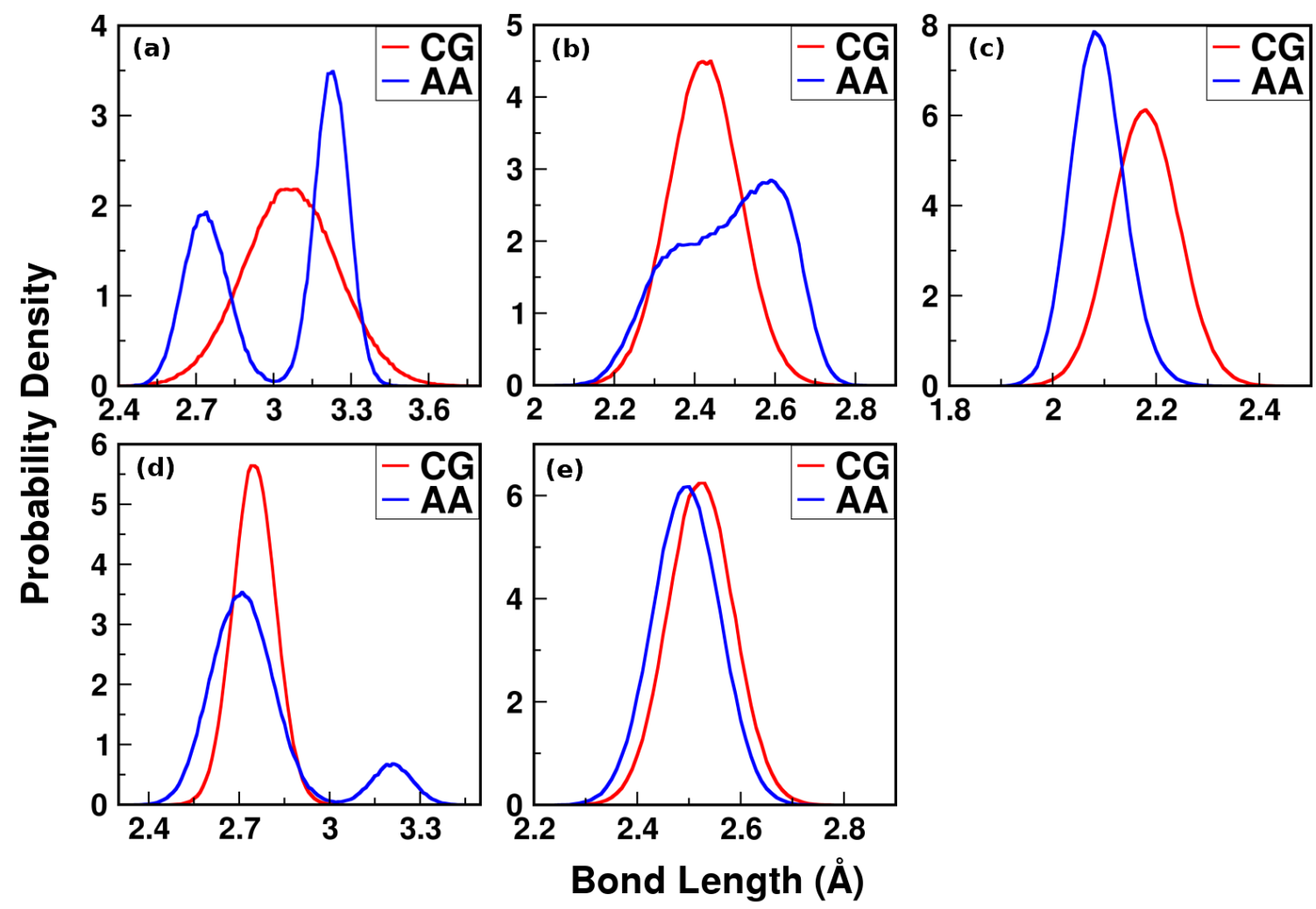

Figure C4.3: Distributions of bonds (a) COO2-NCT, (b) NCT-CO, (c) CO-NC, (d) NC-COO2, and (e) NCCOO1
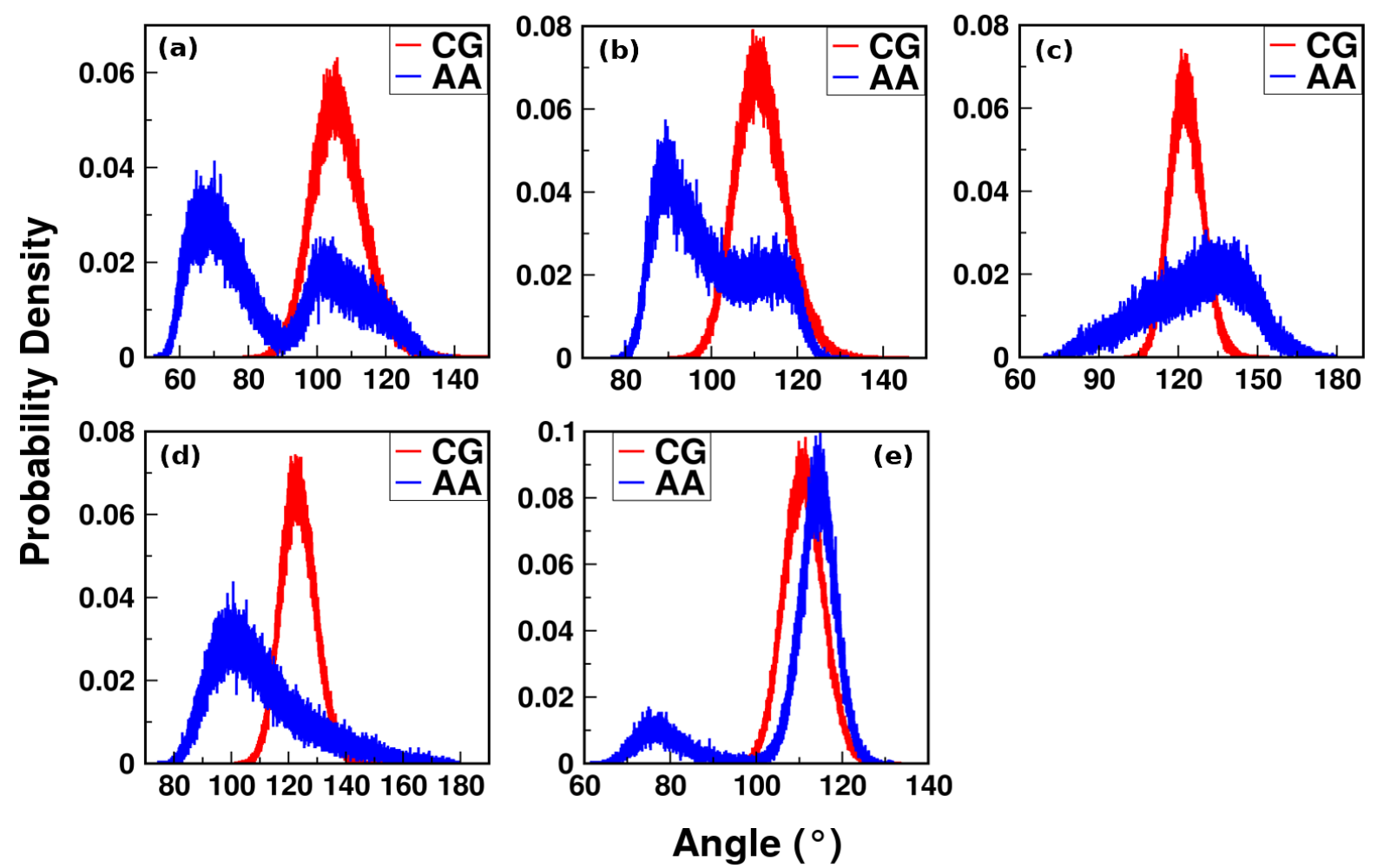

Figure 6.4: Distributions of angles (a) COO2-NCT-CO, (b) NCT-CO-NC, (c) CO-NC-COO2, (d) CO-NCCOO1, and (e) COO2-NC-COO1 

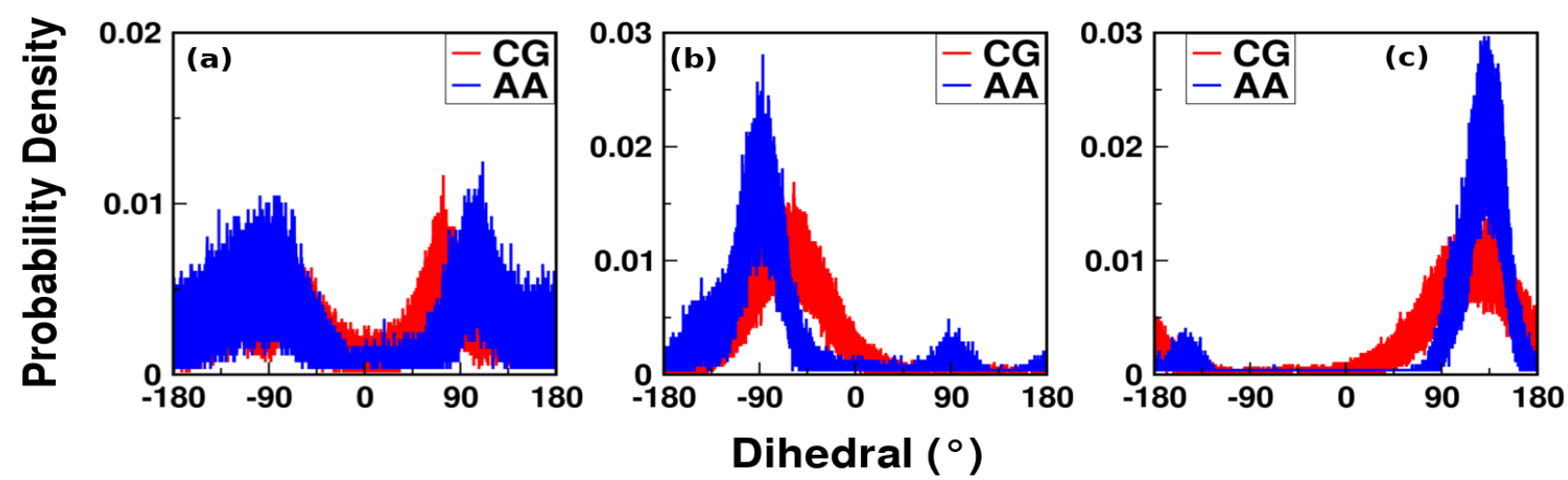

Figure C4.5: Distributions of dihedrals (a) COO2-NCT-CO-NC, (b) NCT-CO-NC-COO2, and (c) NCT-CONC-COO1

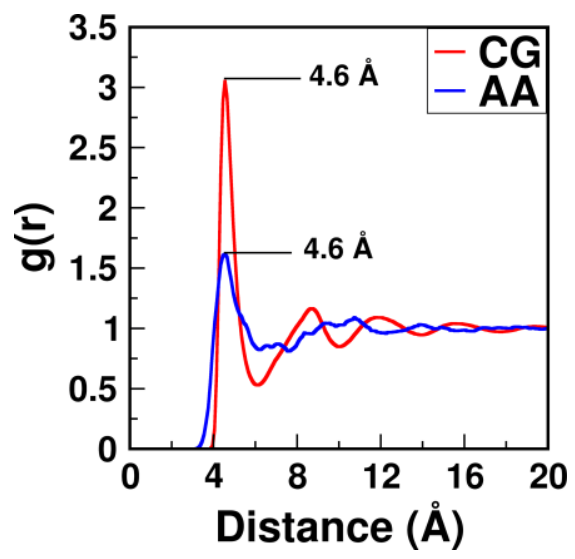

Figure C4.6: $\mathrm{RDF}$ of $\mathrm{COO} 2$

Analogue: Acetic Acid<smiles>CC(=O)O</smiles>

Figure C4.7: All-atom acetic acid

\section{$\mathrm{COO2}$}

Figure C4.8: CG model of acetic acid showing bead type 


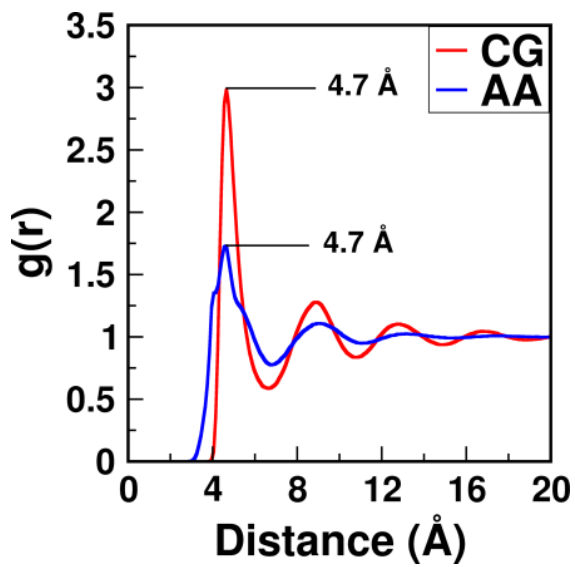

Figure C4.9: $\mathrm{RDF}$ of $\mathrm{COO} 2$ 


\section{C5: Backbone \& Termini}

Backbone Carbonyl Analogue: Diethylketone<smiles>CCC(=O)C(C)CC</smiles>

Figure C5.1: All-atom diethylketone with atom numbering

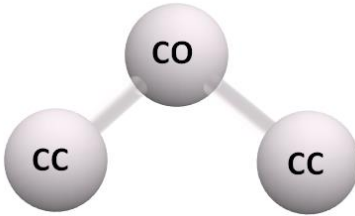

Figure C5.2: CG model of diethylketone showing bead types

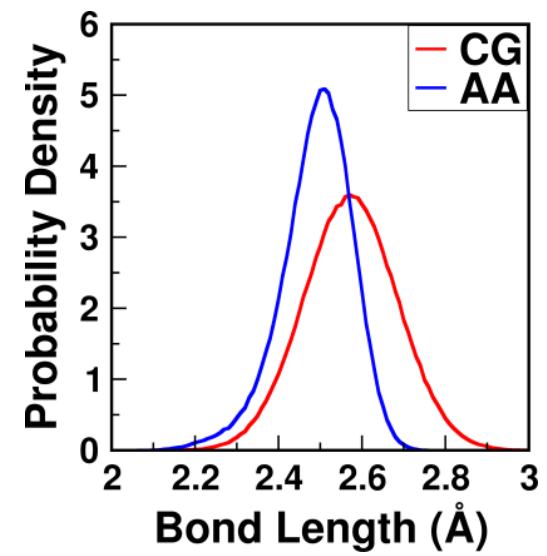

Figure C5.3: Distribution of bond CC-CO

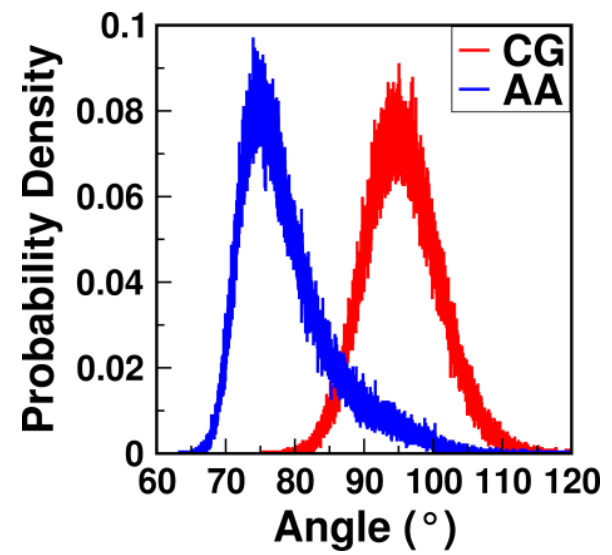

Figure C5.4: Distribution of angle CC-CO-CC 


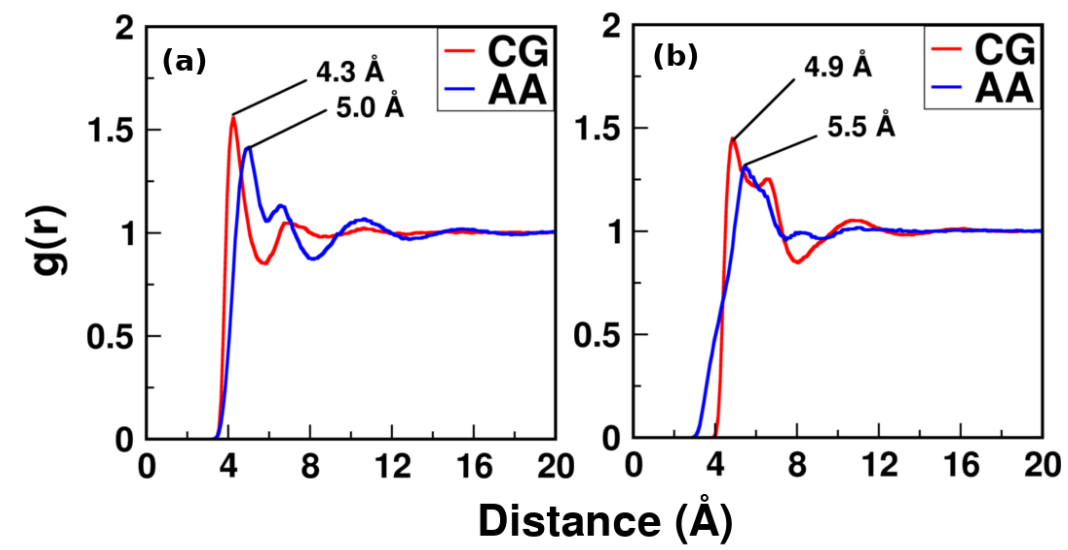

Figure C5.5: RDFs of (a) CC and (b) CO

Backbone N-C $\alpha$ Analogue: Methylacetamide

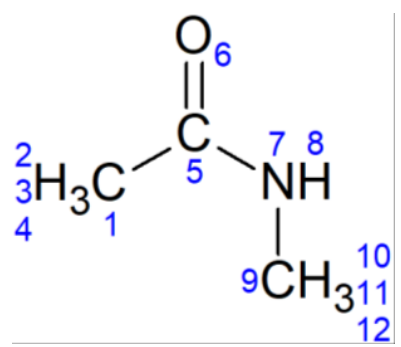

Figure C5.6: All-atom N-methylacetamide with atom numbering

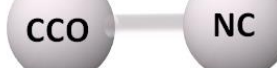

Figure C5.7: CG model of N-methylacetamide showing bead types

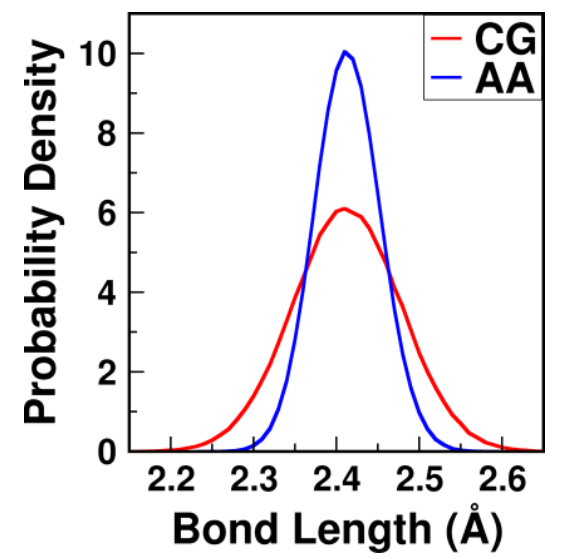

Figure C5.8: Distribution of bond NC-CCO 


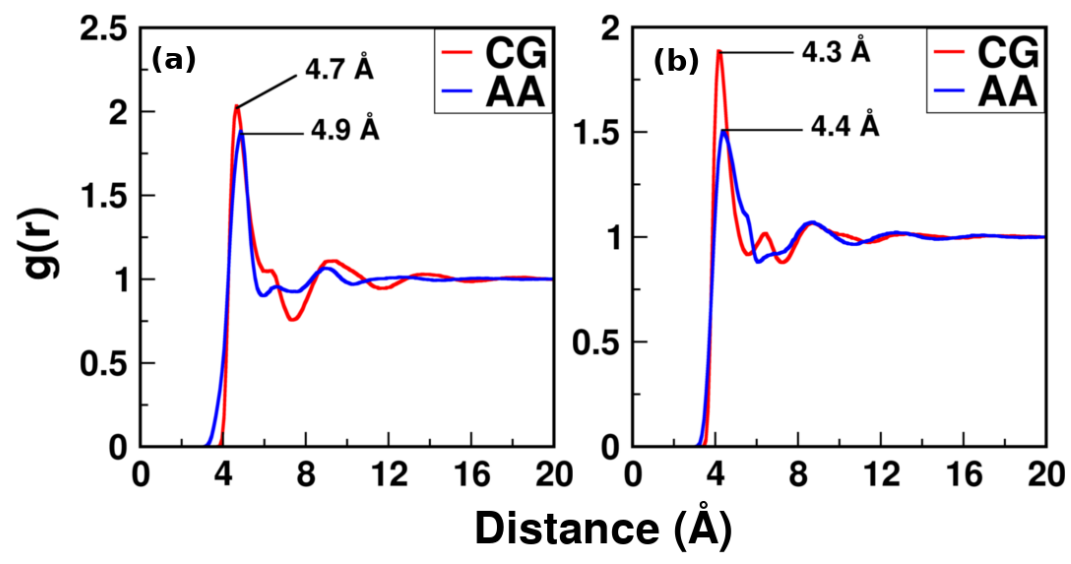

Figure C5.9: RDFs of (a) CCO and (b) NC 


\section{C6: Cysteine}

\section{Cys-Cys Dipeptide}

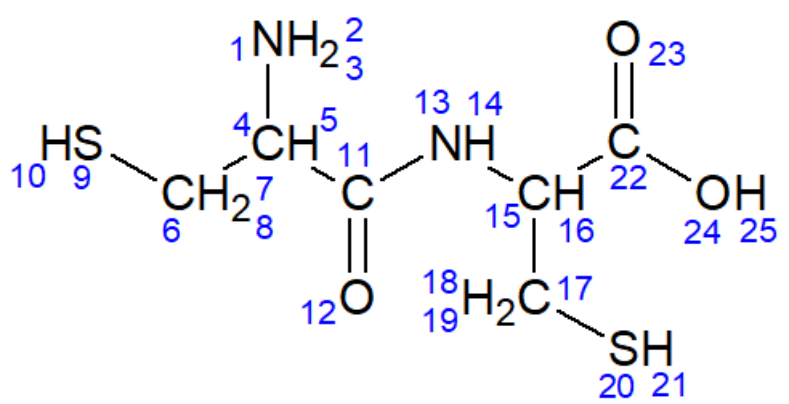

Figure C6.1: All-atom Cysteine dipeptide with atom numbering

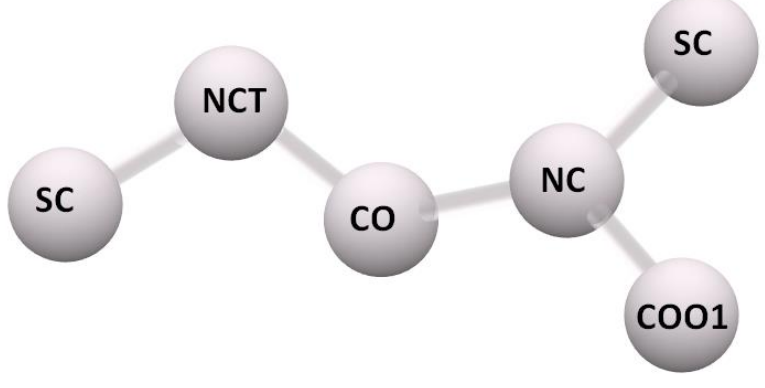

Figure C6.2: CG model of Cysteine dipeptide showing bead types 

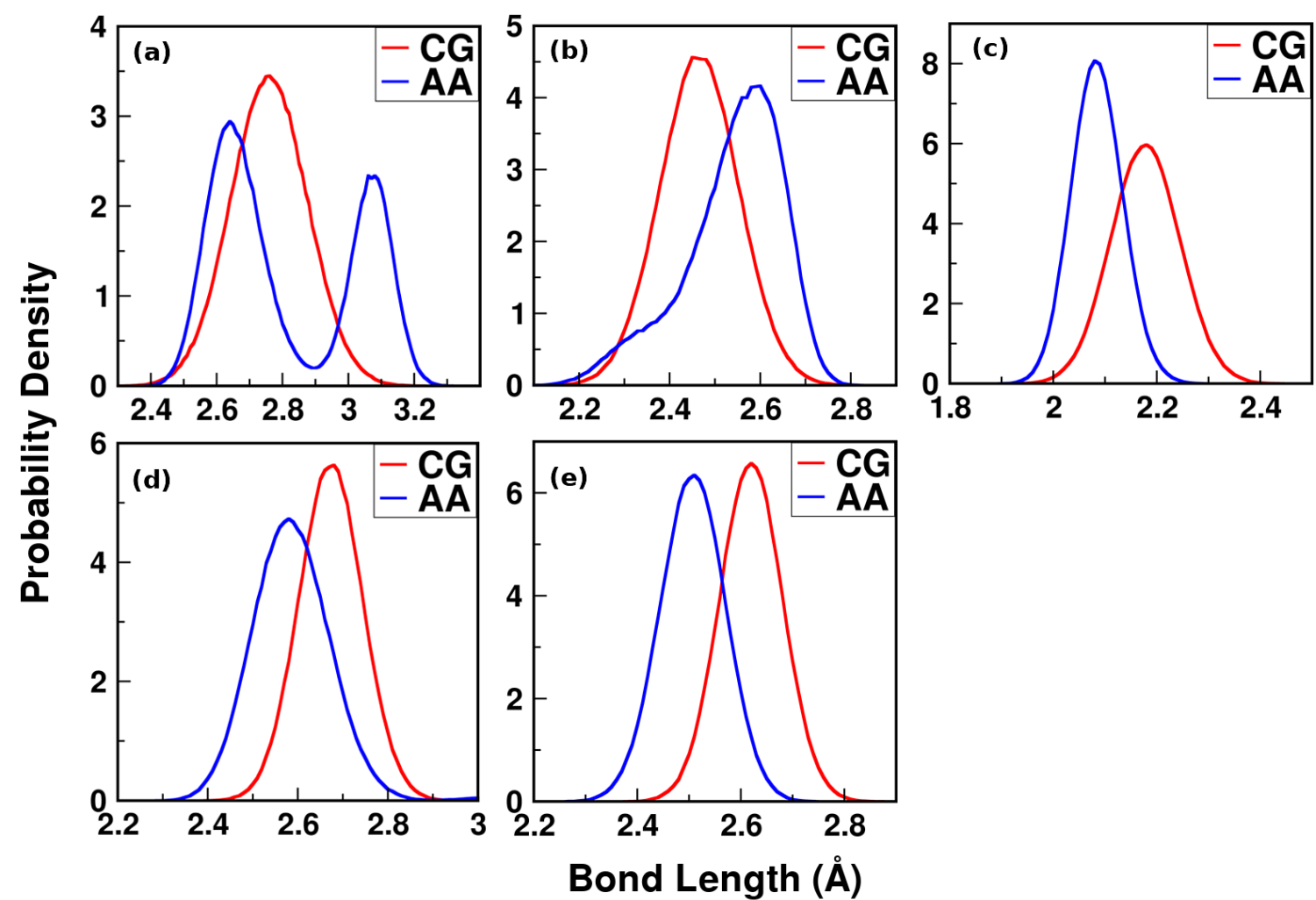

Figure C6.3: Distributions of bonds (a) SC-NCT, (b) NCT-CO, (c) CO-NC, (d) NC-SC, and (e) NC-COO1
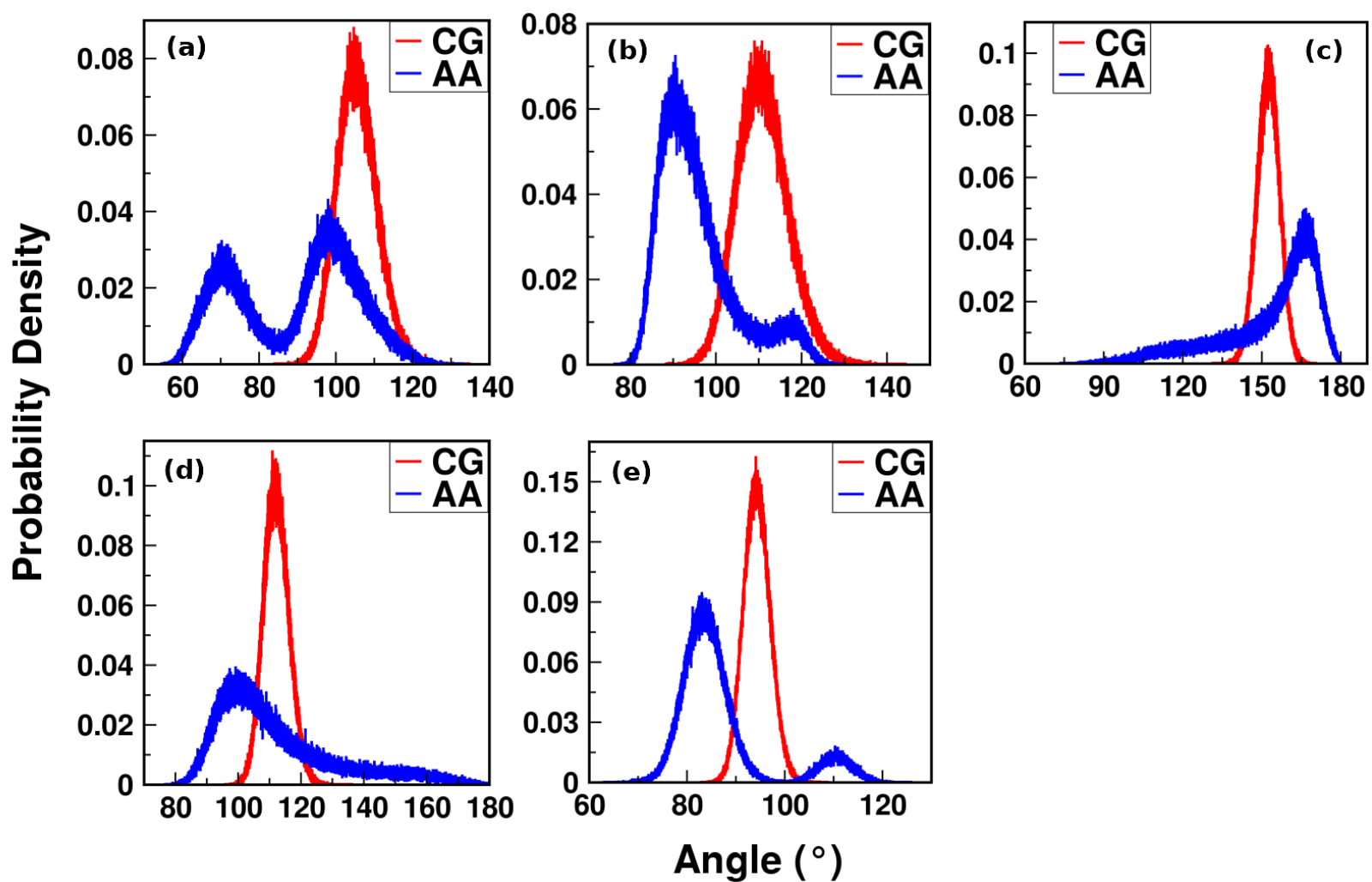

Figure C6.4: Distributions of angles (a) SC-NCT-CO, (b) NCT-CO-NC, (c) CO-NC-SC, (d) CO-NC-COO1, and (e) SC-NC-COO1 

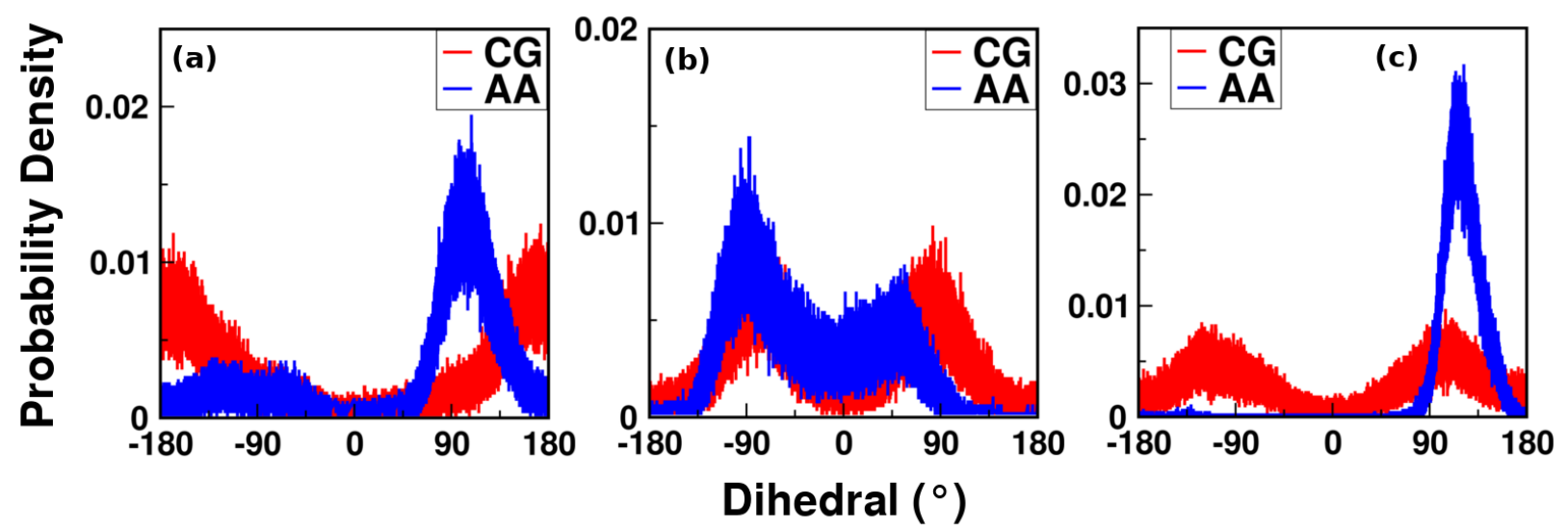

Figure C6.5: Distributions of dihedrals (a) SC-NCT-CO-NC, (b) NCT-CO-NC-SC, and (c) NCT-CO-NCCOO1

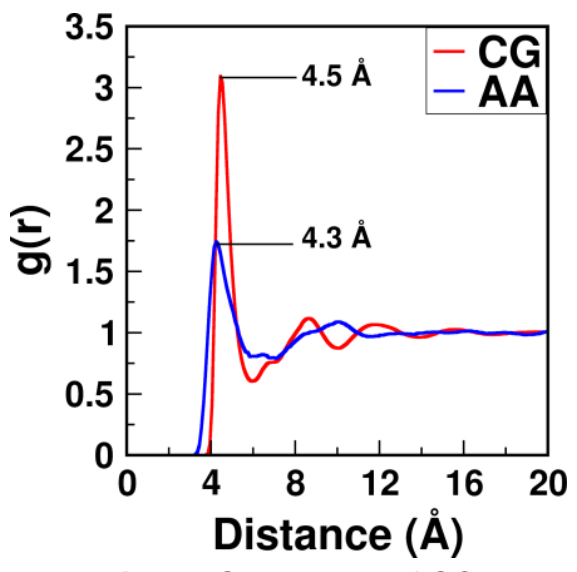

Figure C6.6: RDF of SC

Analogue: Methanethiol<smiles>CS</smiles>

Figure C6.7: All-atom methanethiol

\section{SC}

Figure C6.8: CG model of methanethiol showing bead type 


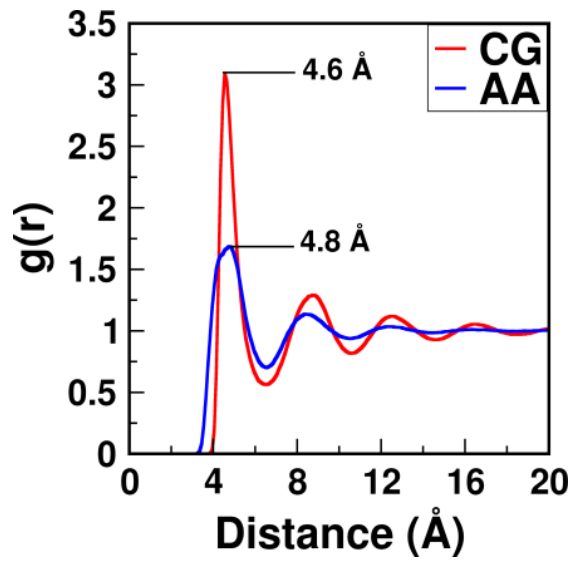

Figure C6.9: RDF of SC 


\section{C7: Glutamic Acid}

\section{Glu-Glu Dipeptide}

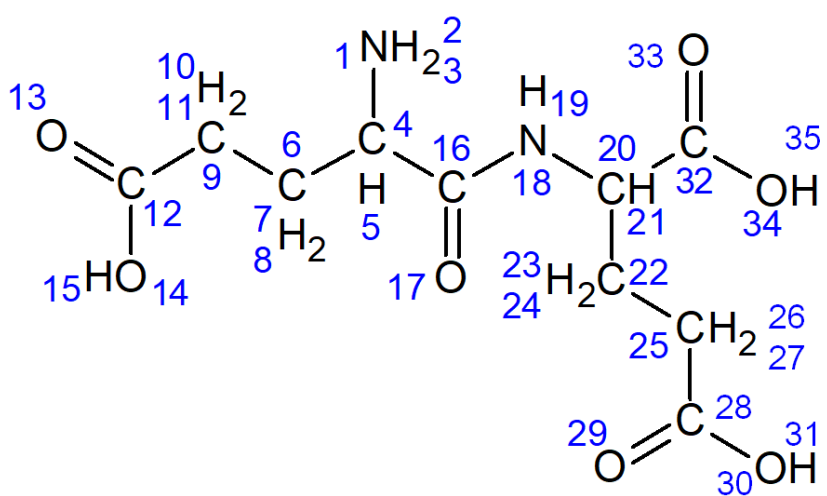

Figure C7.1: All-atom Glutamic Acid dipeptide with atom numbering

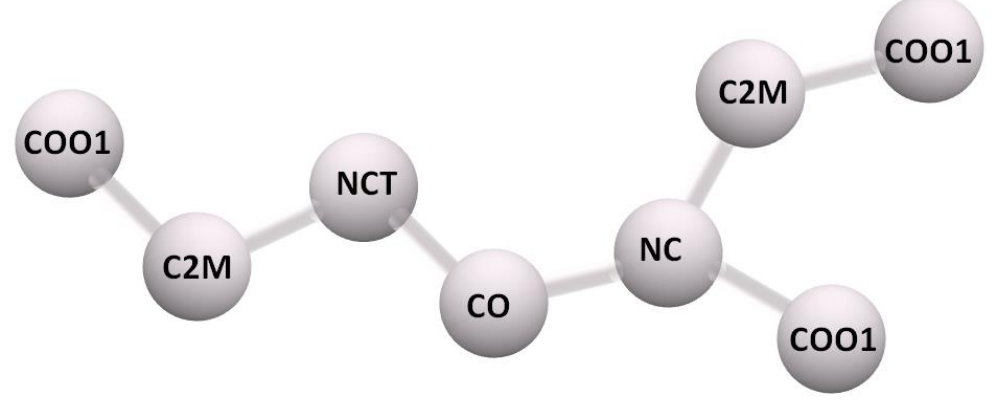

Figure C7.2: CG model of Glutamic Acid dipeptide showing bead types 

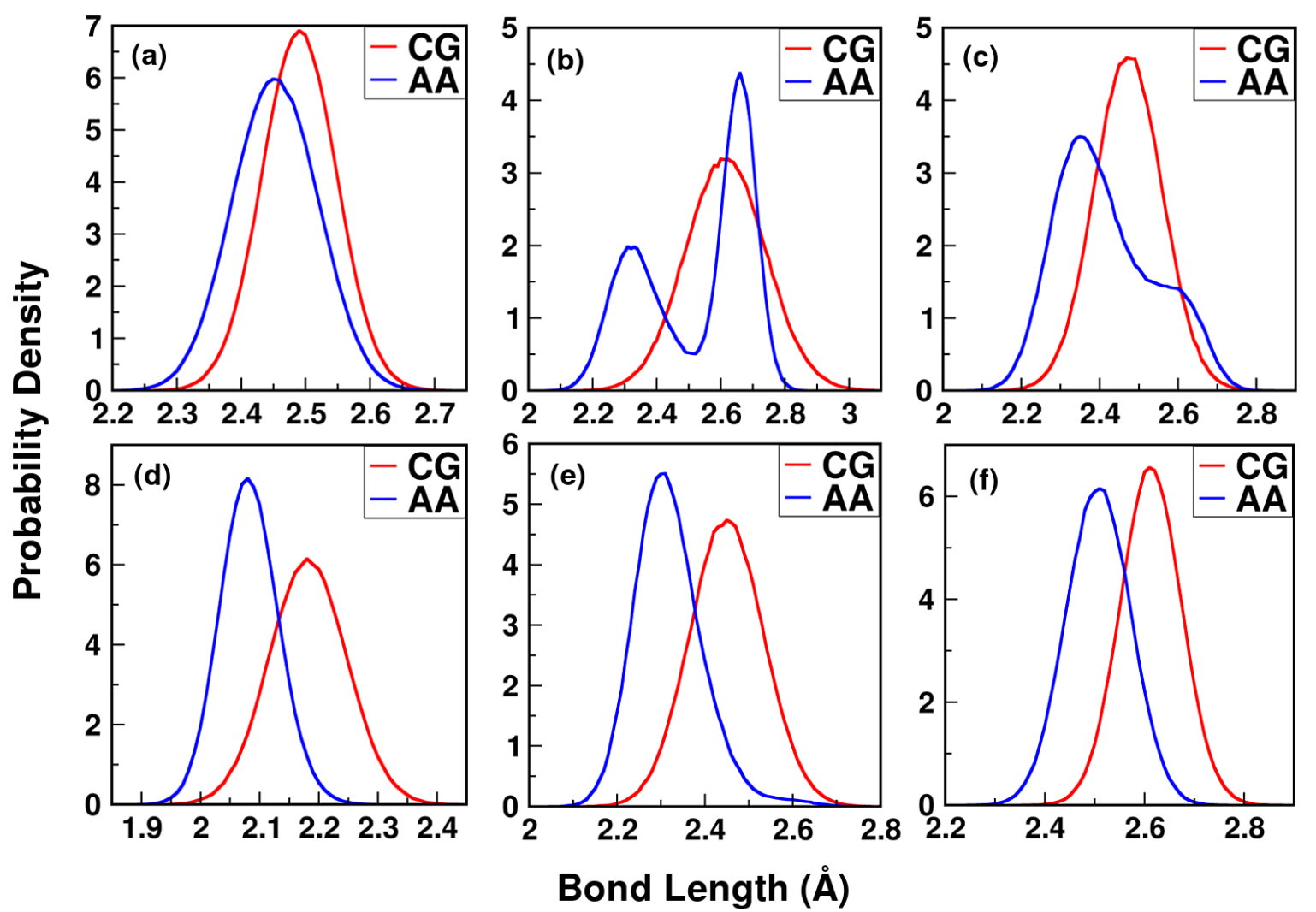

Figure C7.3: Distributions of bonds (a) COO1-C2M, (b) C2M-NCT, (c) NCT-CO, (d) CO-NC, (e) NC-C2M, and (f) NC-COO1 

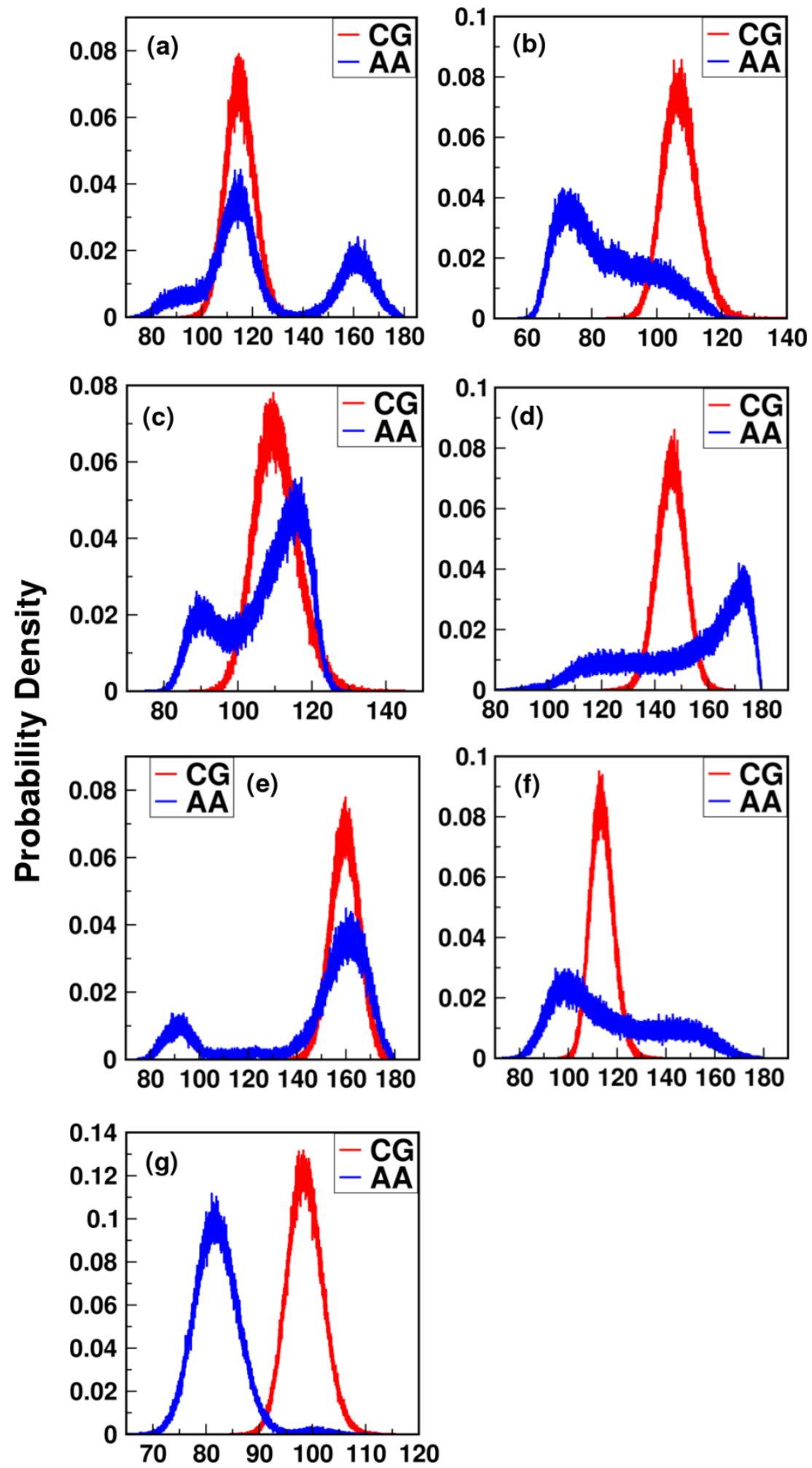

\section{Angle $\left({ }^{\circ}\right)$}

Figure C7.4: Distributions of angles (a) COO1-C2M-NCT, (b) C2M-NCT-CO, (c) NCT-CO-NC, (d) CO$\mathrm{NC}-\mathrm{C} 2 \mathrm{M}$, (e) NC-C2M-COO1, (f) CO-NC-COO1, and (g) C2M-NC-COO1 

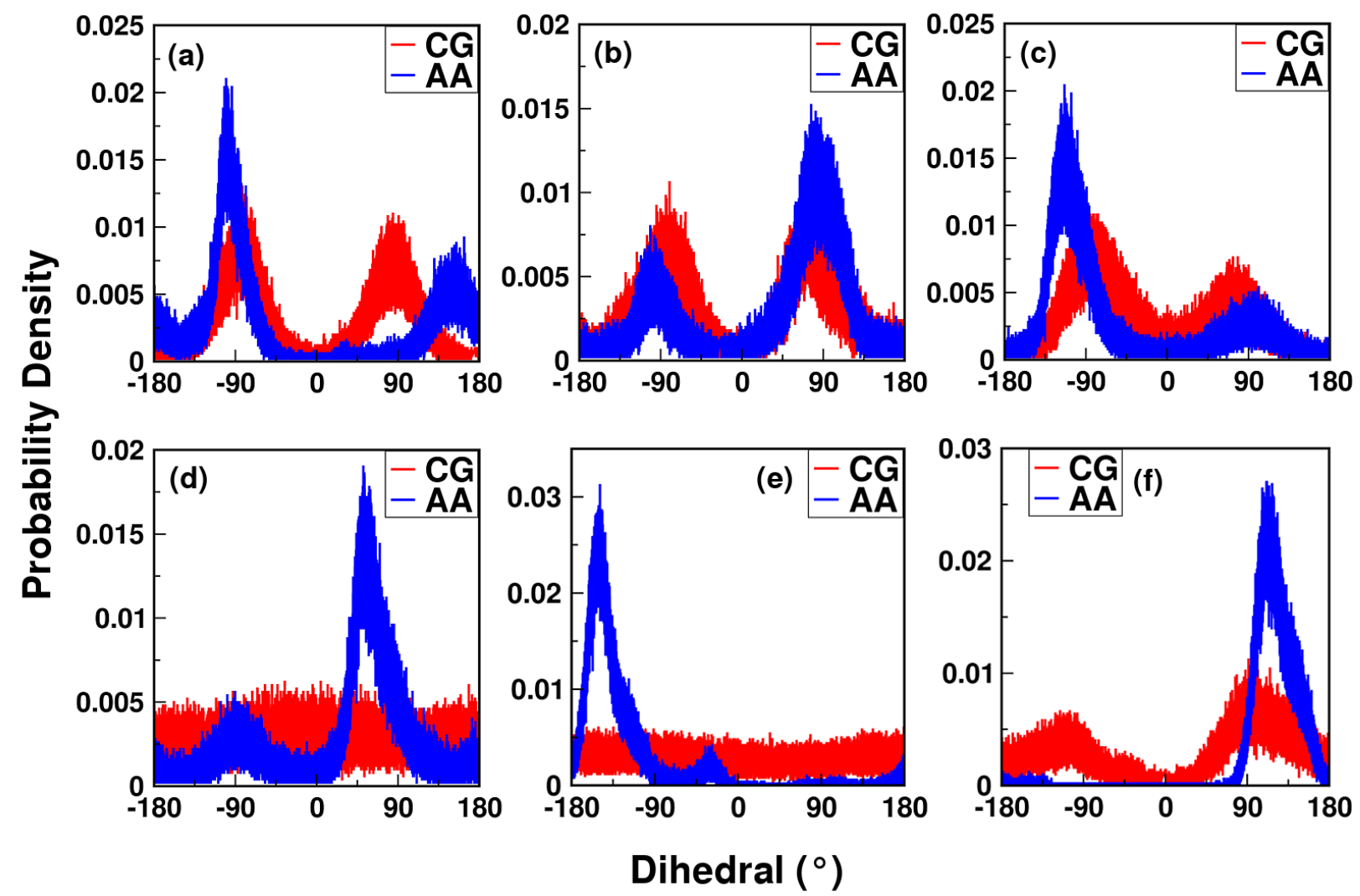

Figure C7.5: Distributions of dihedrals (a) COO1-C2M-NCT-CO, (b) C2M-NCT-CO-NC, (c) NCT-CO-NCC2M, (d) CO-NC-C2M-COO1, (e) COO1-C2M-NC-COO1, and (f) NCT-CO-NC-COO1

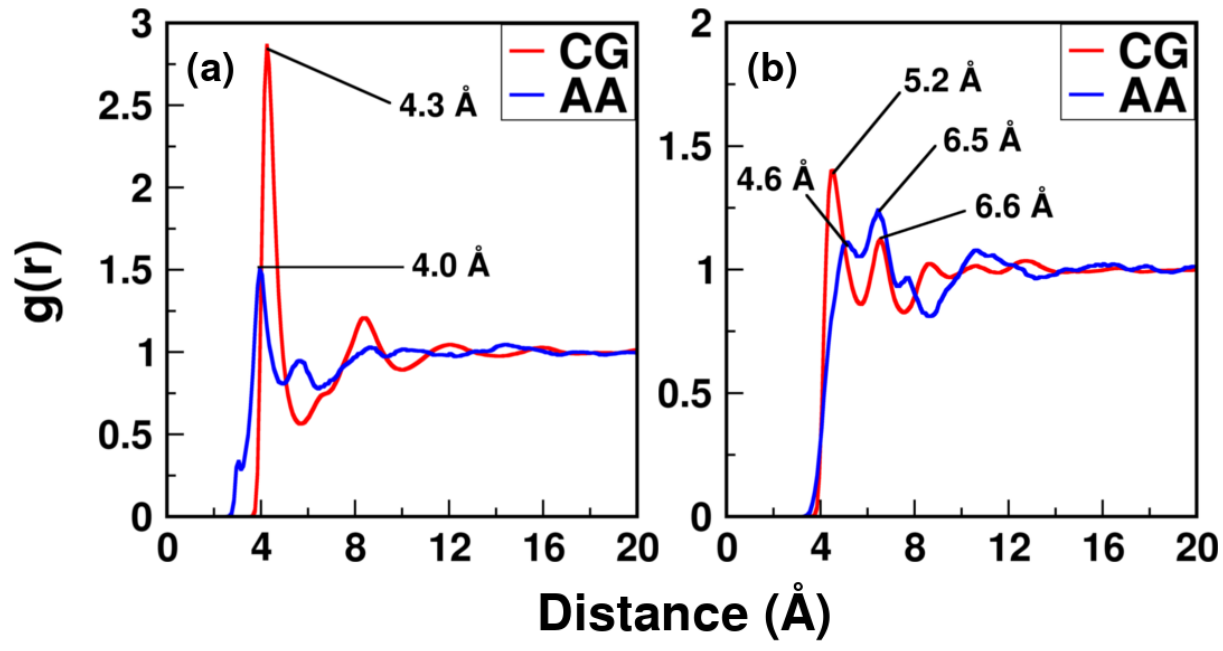

Figure C7.6: RDFs of (a) COO1 and (b) C2M 


\section{C8: Glutamine}

\section{Gln-Gln Dipeptide}

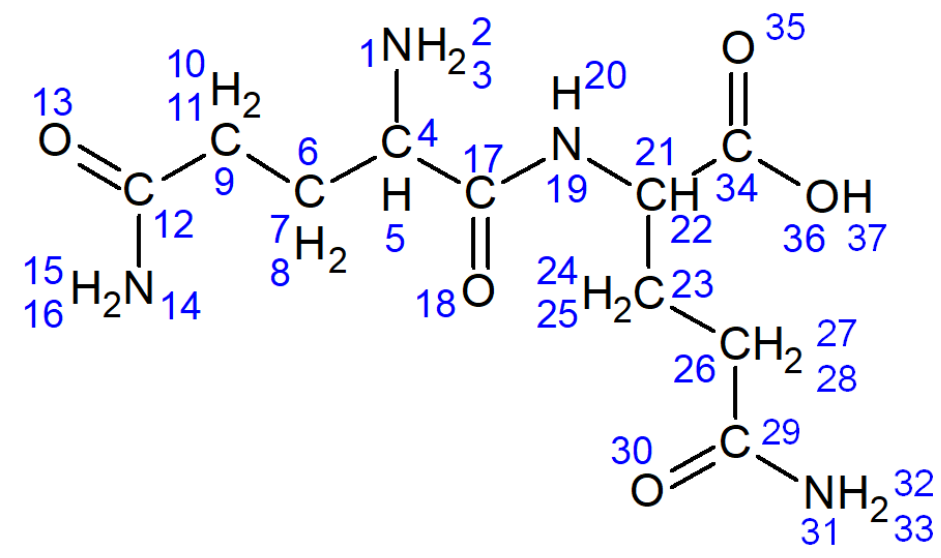

Figure C8.1: All-atom Glutamine dipeptide with atom numbering

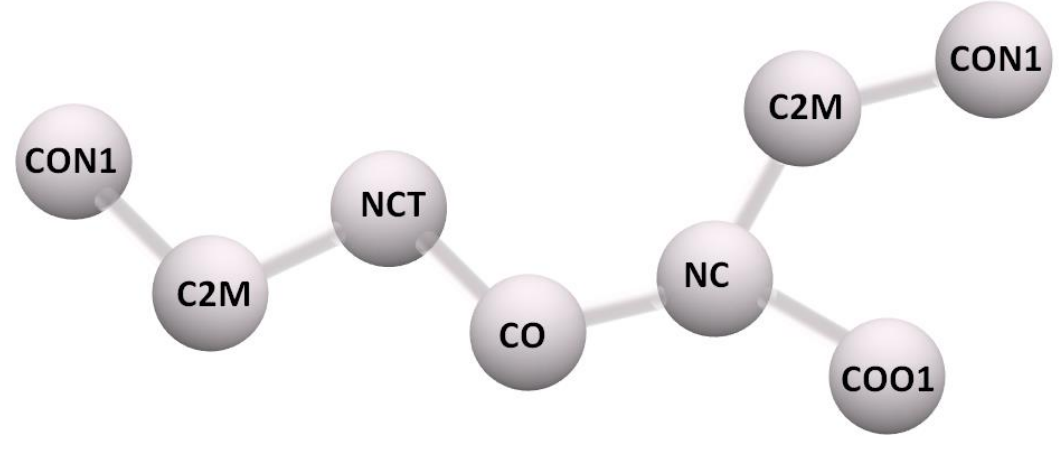

Figure C8.2: CG model of Glutamine dipeptide showing bead types 

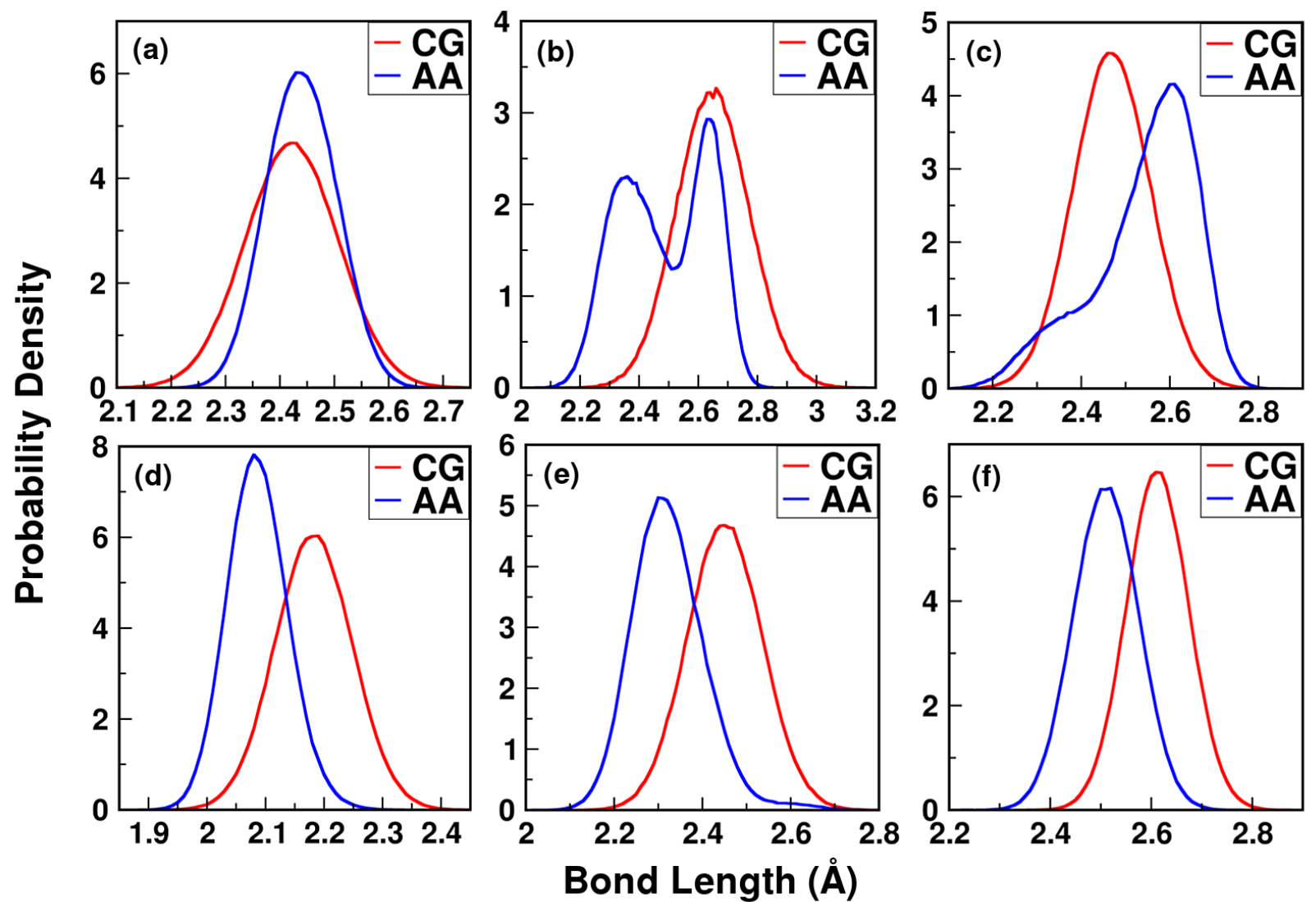

Figure C8.3: Distributions of bonds (a) CON1-C2M, (b) C2M-NCT, (c) NCT-CO, (d) CO-NC, (e) NC-C2M, and (f) NC-COO1 

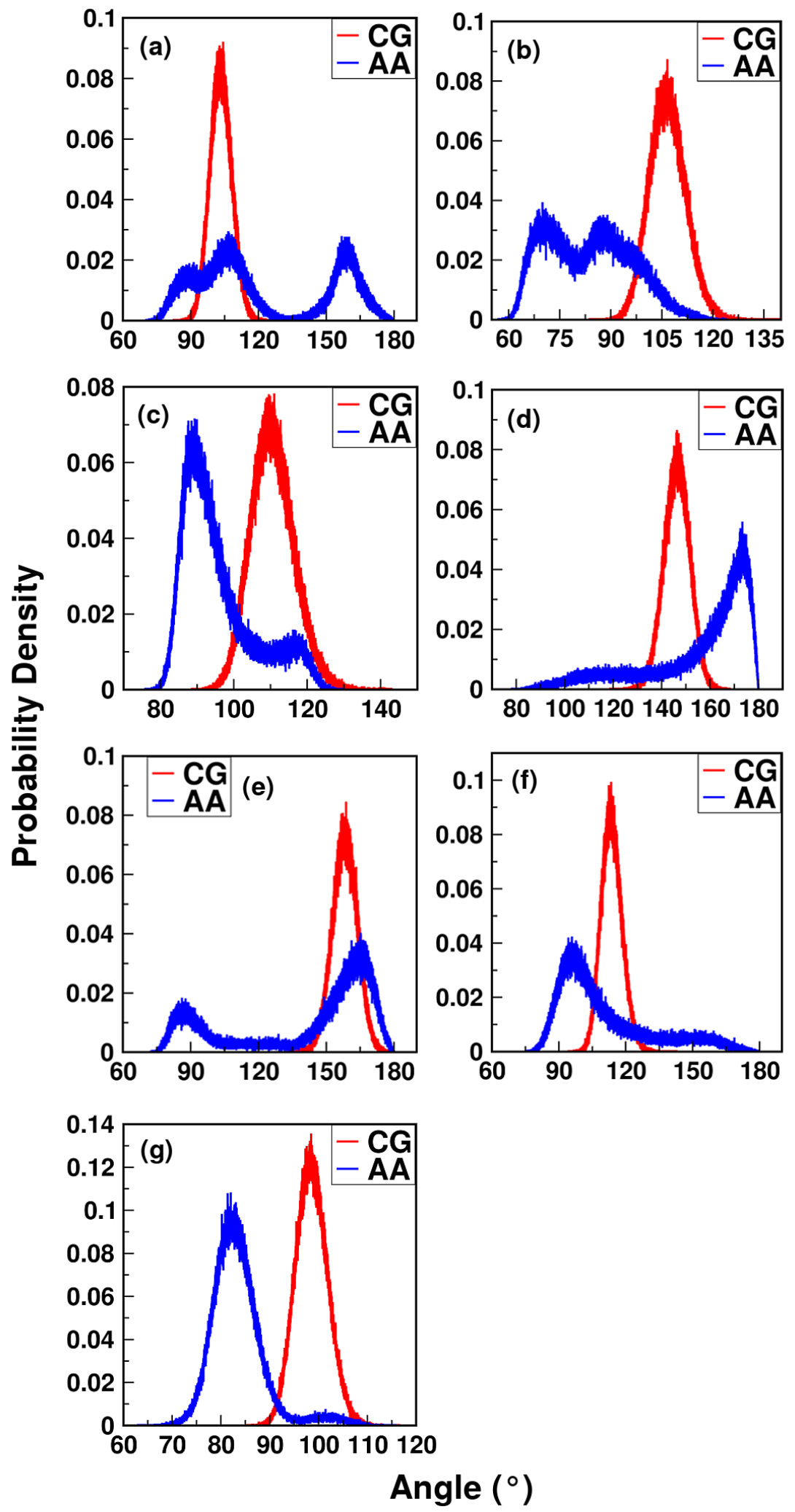

Figure C8.4: Distributions of angles (a) CON1-C2M-NCT, (b) C2M-NCT-CO, (c) NCT-CO-NC, (d) CONC-C2M, (e) NC-C2M-CON1, (f) CO-NC-COO1, and (g) C2M-NC-COO1 

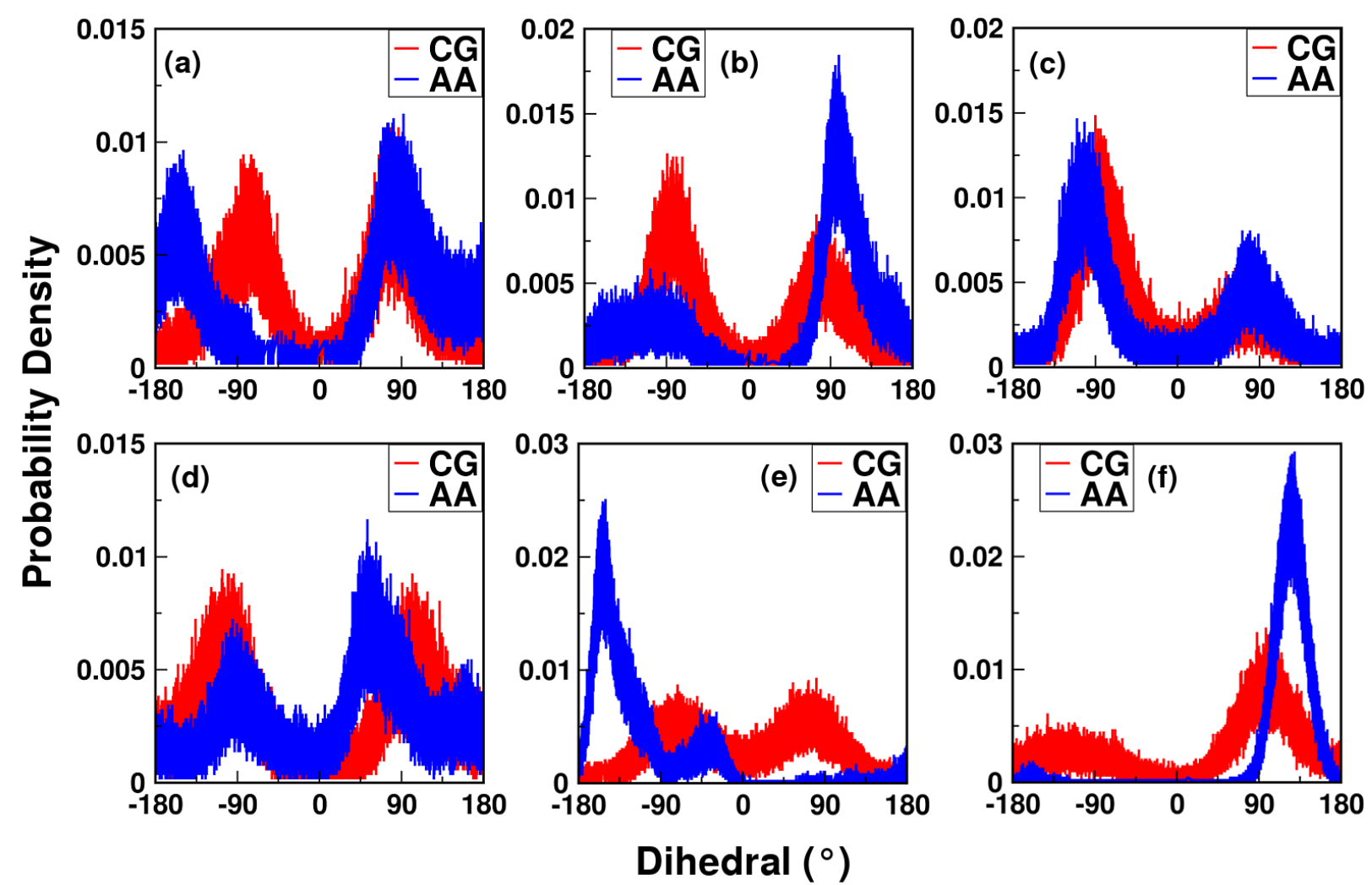

Figure C8.5: Distributions of dihedrals (a) CON1-C2M-NCT-CO, (b) C2M-NCT-CO-NC, (c) NCT-CO-NCC2M, (d) CO-NC-C2M-CON1, (e) CON1-C2M-NC-COO1, and (f) NCT-CO-NC-COO1

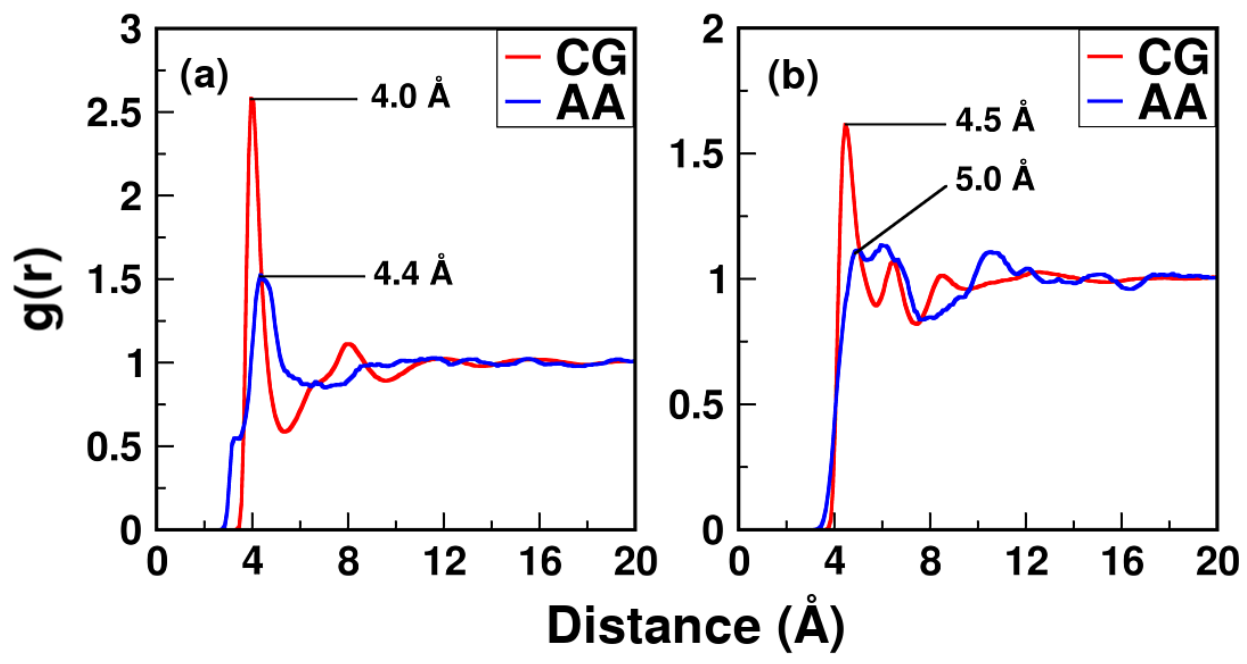

Figure C8.6: RDFs of (a) CON1 and (b) C2M 
Analogue: Propionamide

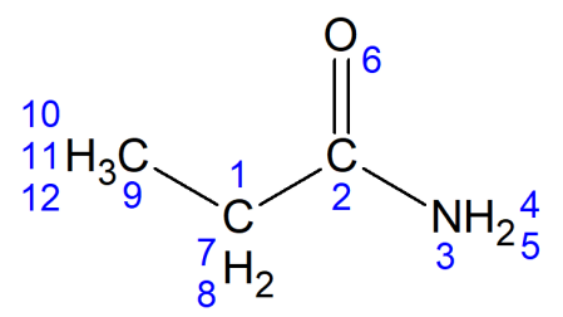

Figure C8.7: All-atom propionamide with atom numbering

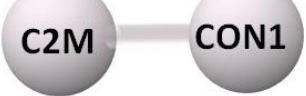

Figure C8.8: CG model of propionamide showing bead type

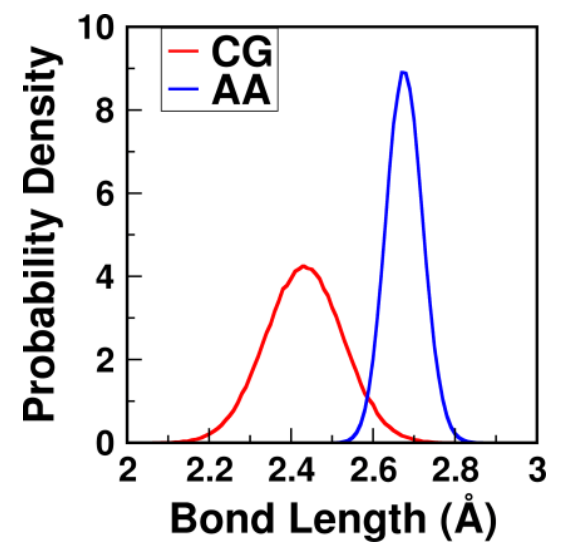

Figure C8.9: Distribution of bond CON1-C2M. The equilibrium bond length from the GInGln dipeptide was used so as to provide a better match to the glutamine side chain.

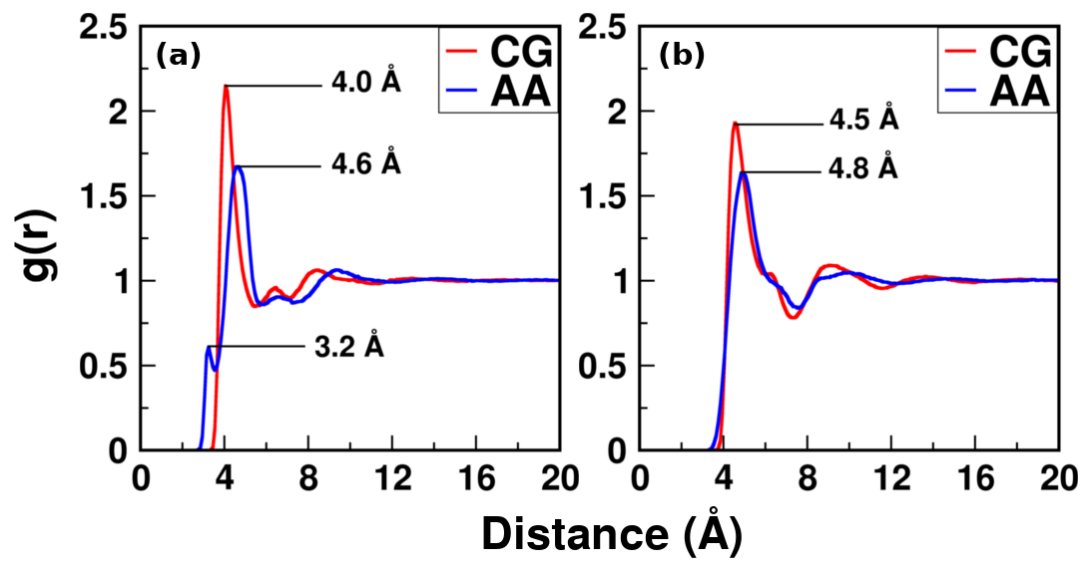

Figure C8.10: RDFs of (a) CON1 and (b) C2M 


\section{C9: Glycine}

\section{Gly-Gly Dipeptide}

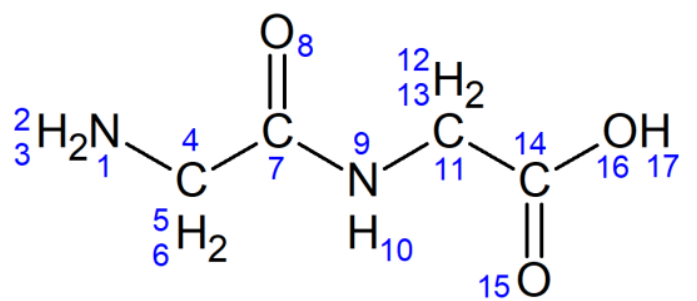

Figure C9.1: All-atom Glycine dipeptide with atom numbering

\section{GNT}

GNC

CO

COO1

Figure C9.2: CG model of Glycine dipeptide showing bead types

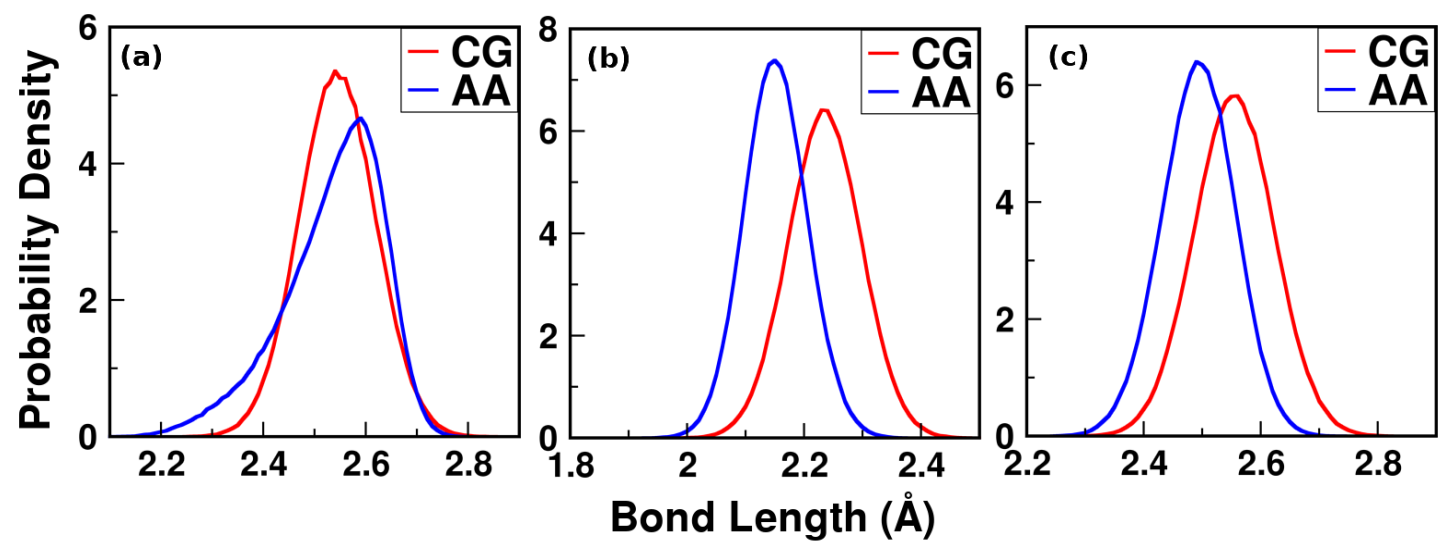

Figure C9.3: Distributions of bonds (a) GNT-CO, (b) CO-GNC, and (c) GNC-COO1

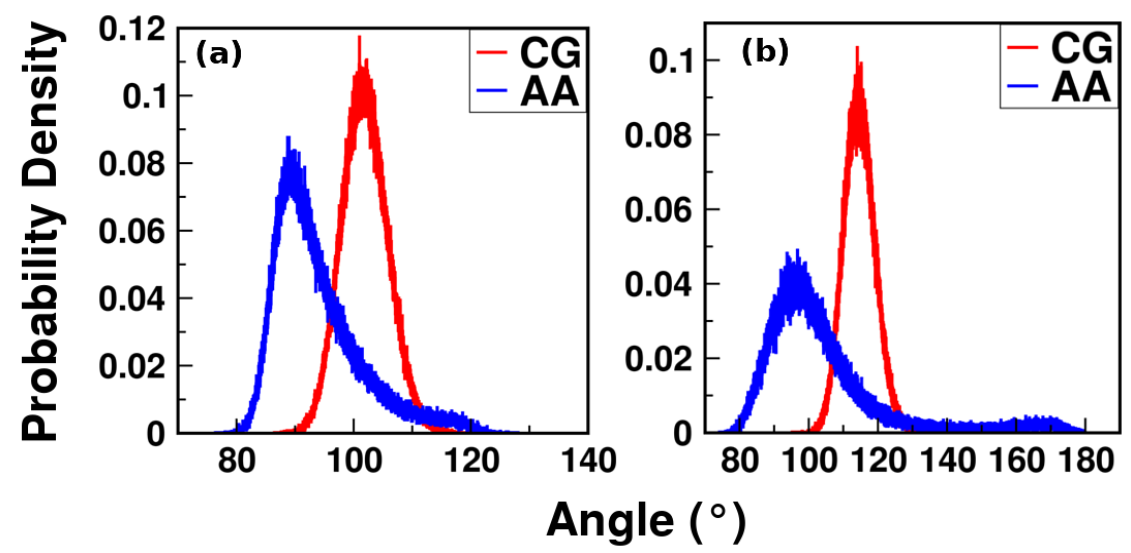

Figure C9.4: Distributions of angles (a) GNT-CO-GNC and (b) CO-GNC-COO1 


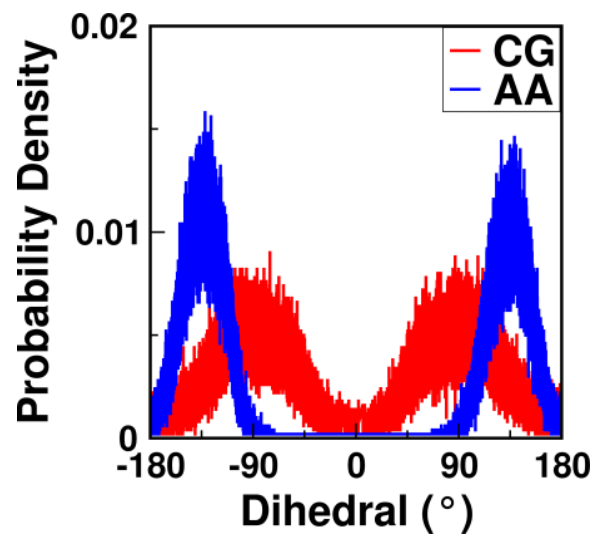

Figure C9.5: Distribution of dihedral GNT-CO-GNC-COO1

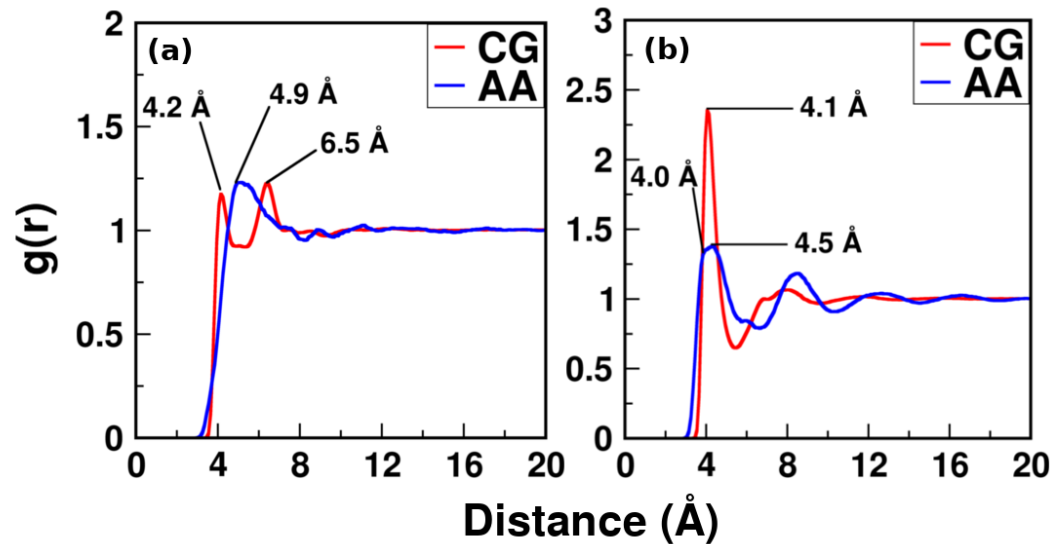

Figure C9.6: RDFs of (a) GNC and (b) GNT 


\section{C10: Histidine}

\section{His-His Dipeptide}

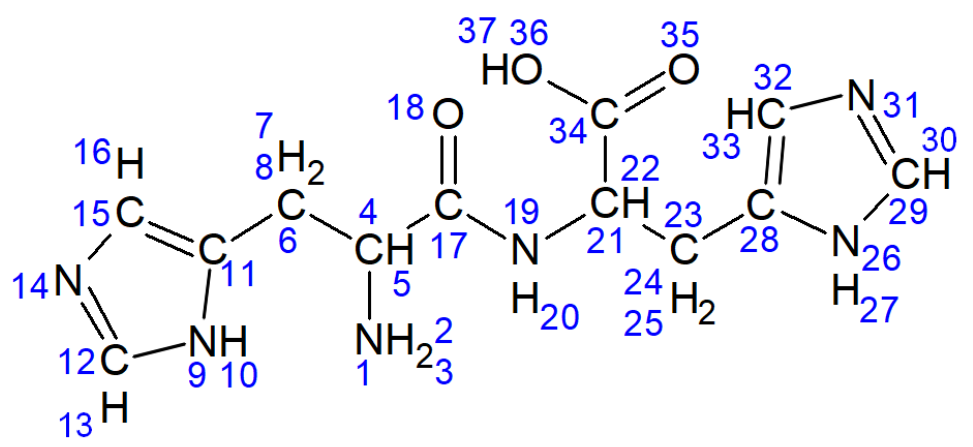

Figure C10.1: All-atom Histidine dipeptide with atom numbering

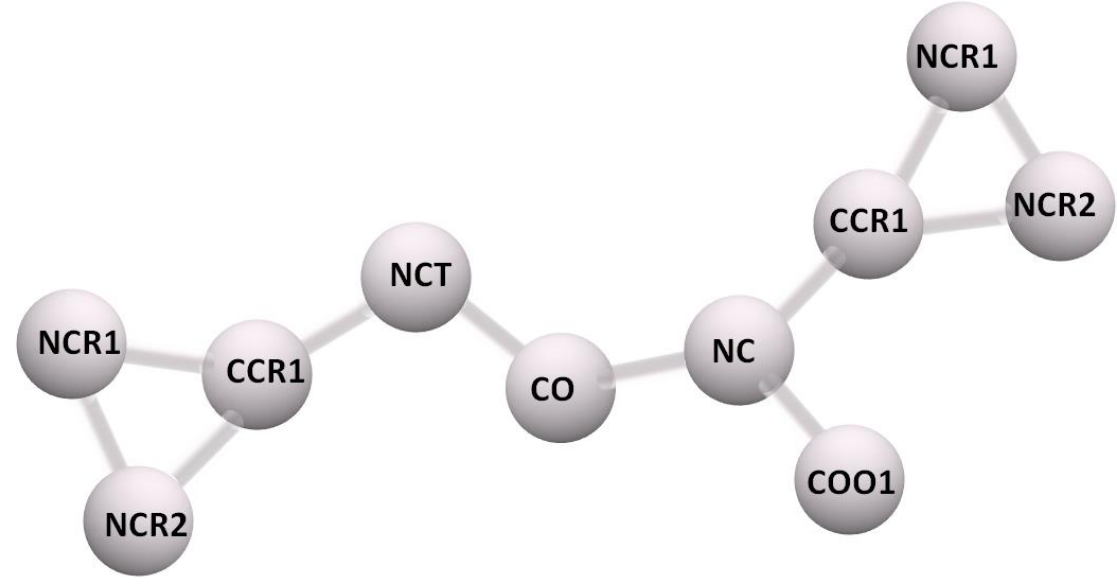

Figure C10.2: CG model of Histidine dipeptide showing bead types 


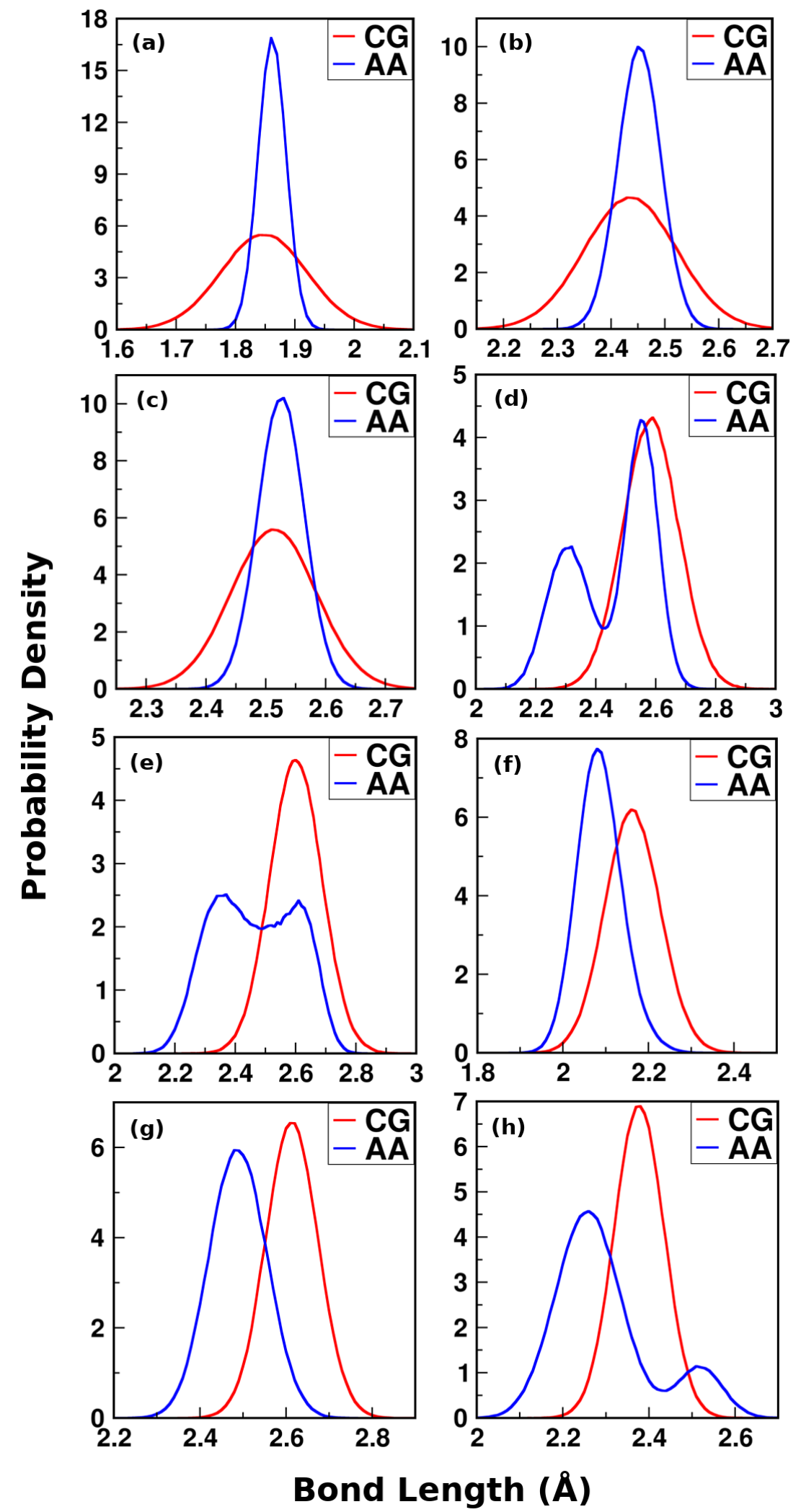

Figure C10.3: Distributions of bonds (a) NCR2-NCR1, (b) NCR1-CCR1, (c) NCR2-CCR1, (d) CCR1NCT, (e) NCT-CO, (f) CO-NC, (g) NC-COO1, and (h) NC-CCR1 

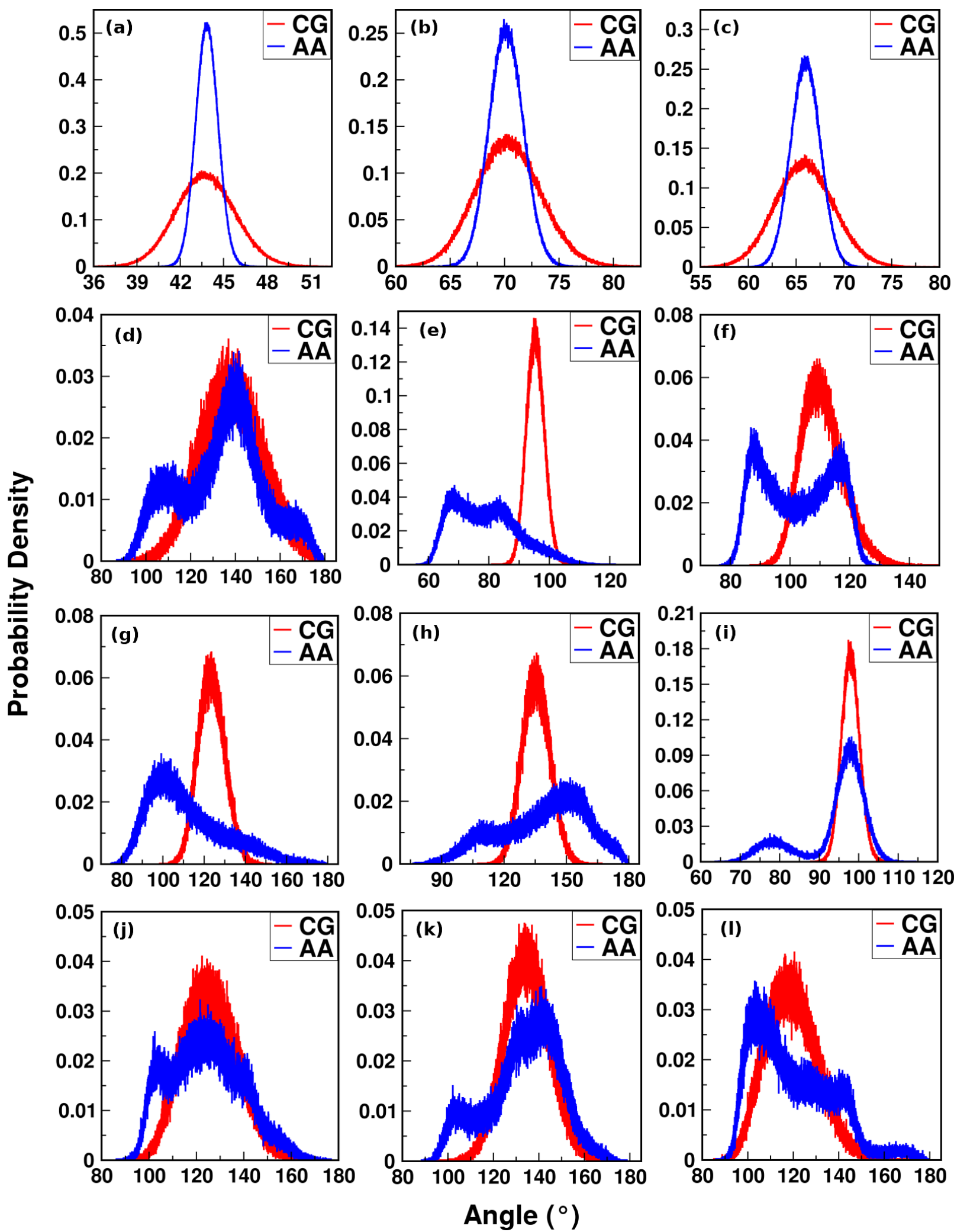

Figure C10.4: Distributions of angles (a) NCR2-CCR1-NCR1, (b) CCR1-NCR1-NCR2, (c) NCR1-NCR2CCR1, (d) NCR1-CCR1-NCT, (e) CCR1-NCT-CO, (f) NCT-CO-NC, (g) CO-NC-COO1, (h) CO-NC-CCR1, (i) COO1-NC-CCR1, (j) NC-CCR1-NCR2, (k) NCR2-CCR1-NCT, and (l) NC-CCR1-NCR1 

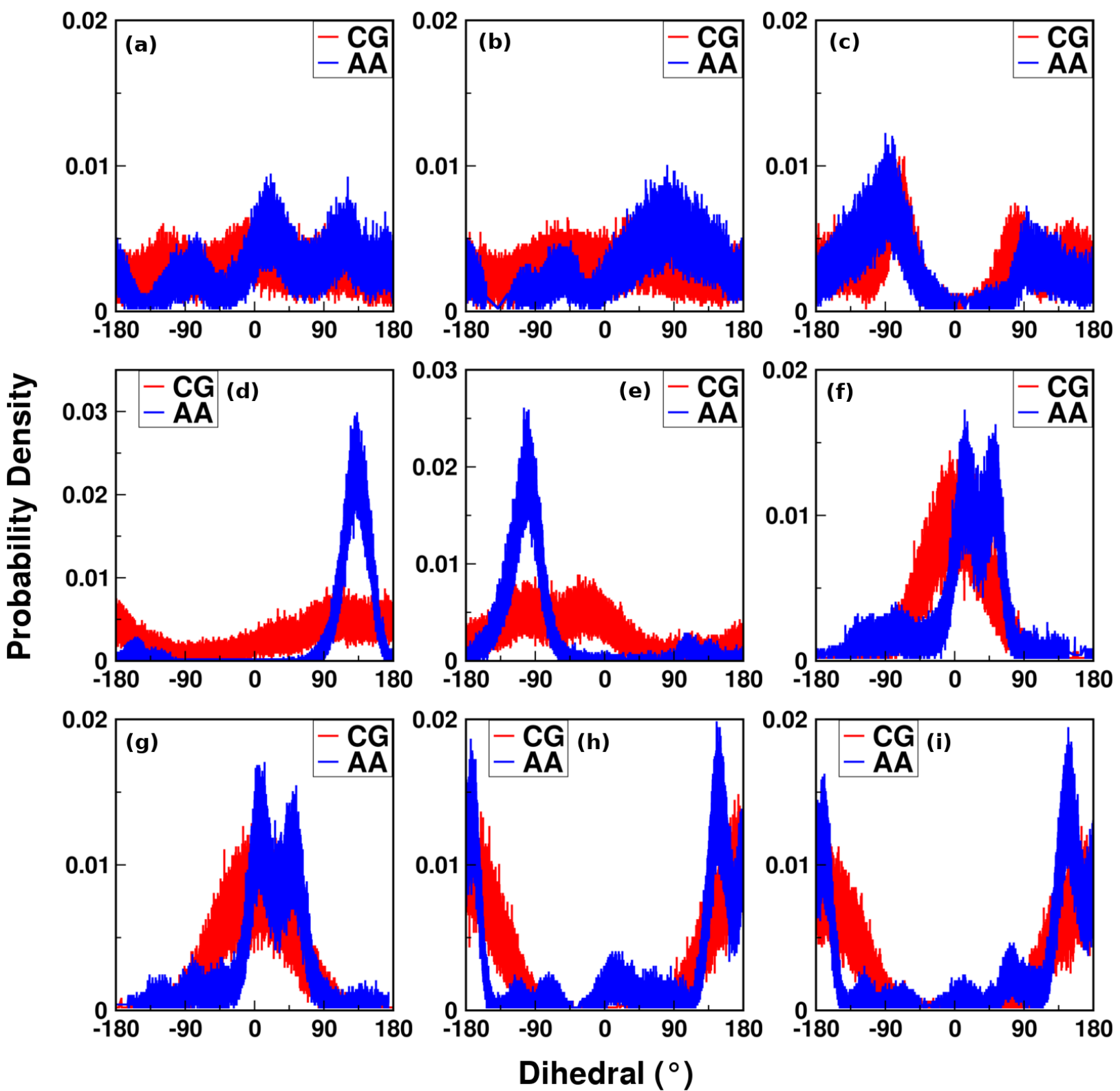

Figure C10.5: Distribution of dihedrals (a) NCR1-CCR1-NCT-CO, (b) NCR2-CCR1-NCT-CO, (c) CCR1NCT-CO-NC, (d) NCT-CO-NC-COO1, (e) NCT-CO-NC-CCR1, (f) CO-NC-CCR1-NCR2, (g) CO-NCCCR1-NCR1, (h) COO1-NC-CCR1-NCR2, and (i) COO1-NC-CCR1-NCR1 

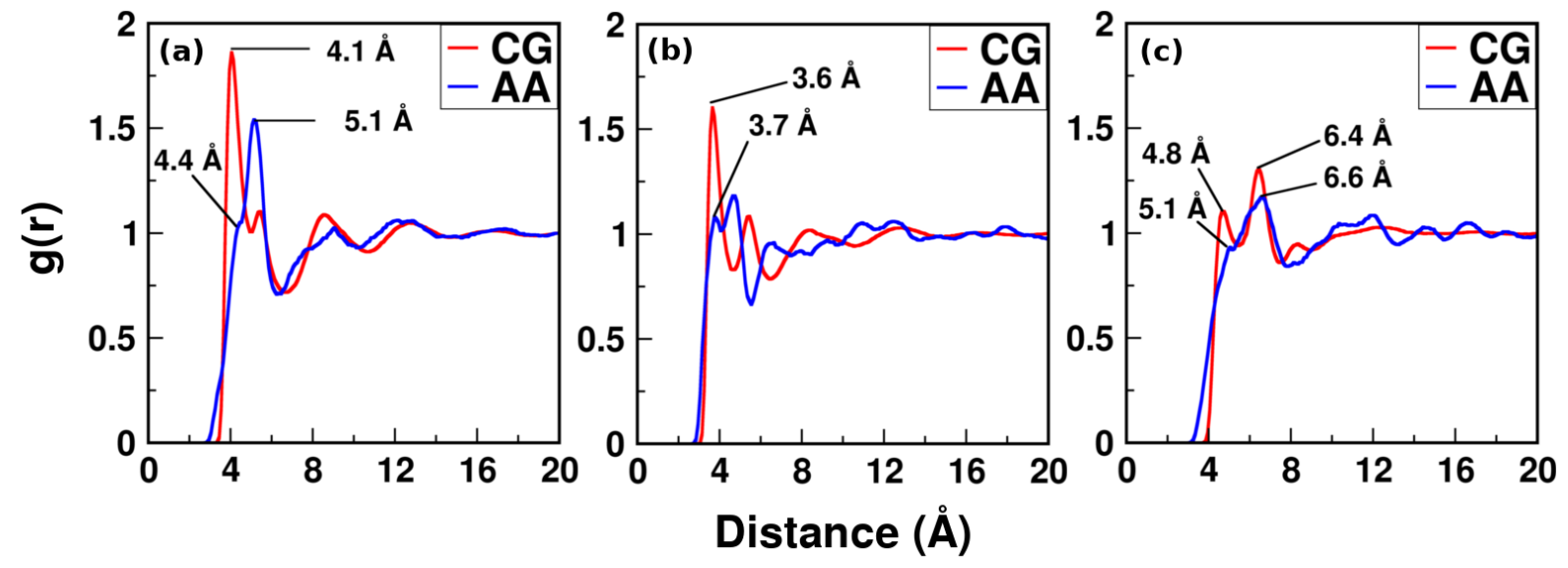

Figure C10.6: RDFs of (a) CCR1, (b) NCR1, and (c) NCR2

Analogue 1: Pyridine

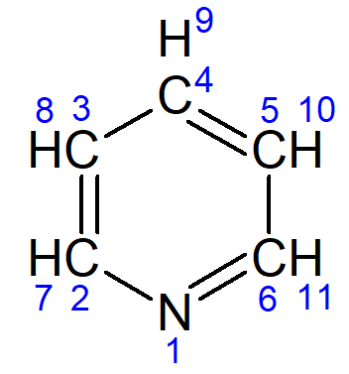

Figure C10.7: All-atom pyridine with atom numbering

BZ

BZ

NCR2

Figure C10.8: CG model of pyridine showing bead types

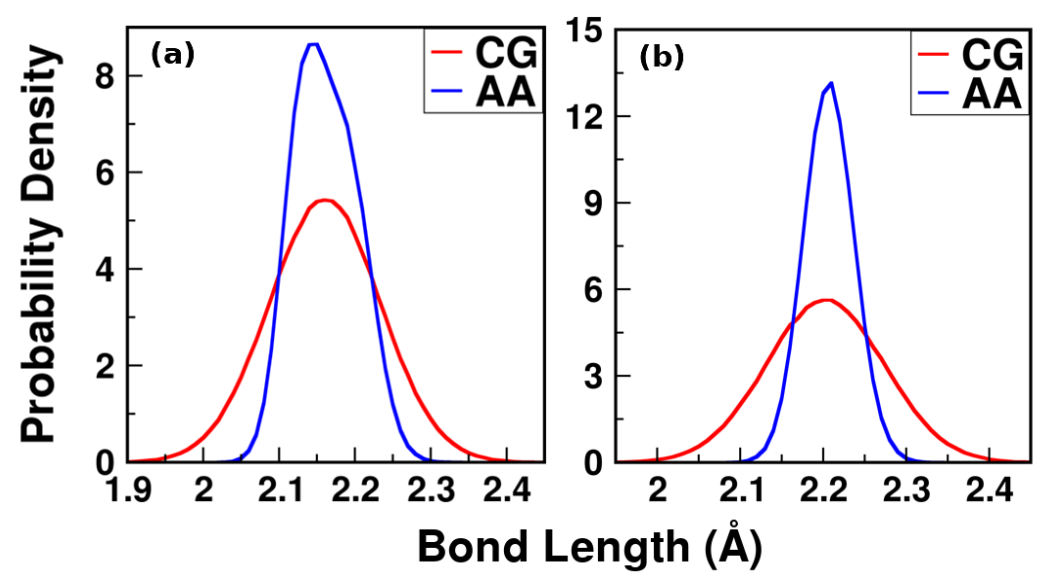

Figure C10.9: Distributions of bonds (a) NCR2-BZ and (b) BZ-BZ 


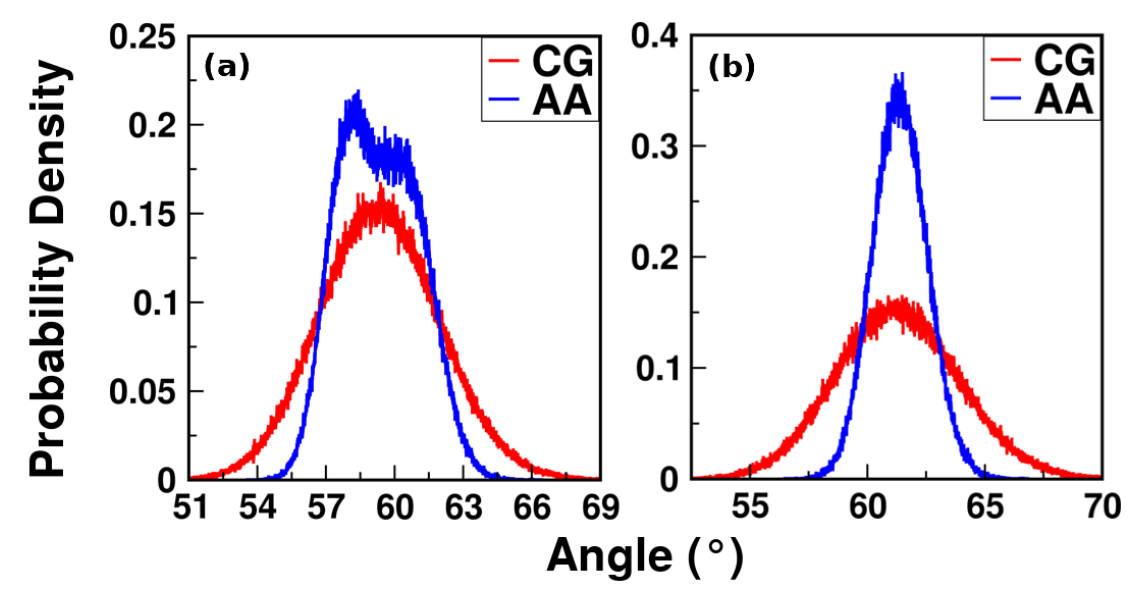

Figure C10.10: Distributions of angles (a) NCR2-BZ-BZ and (b) BZ-NCR2-BZ

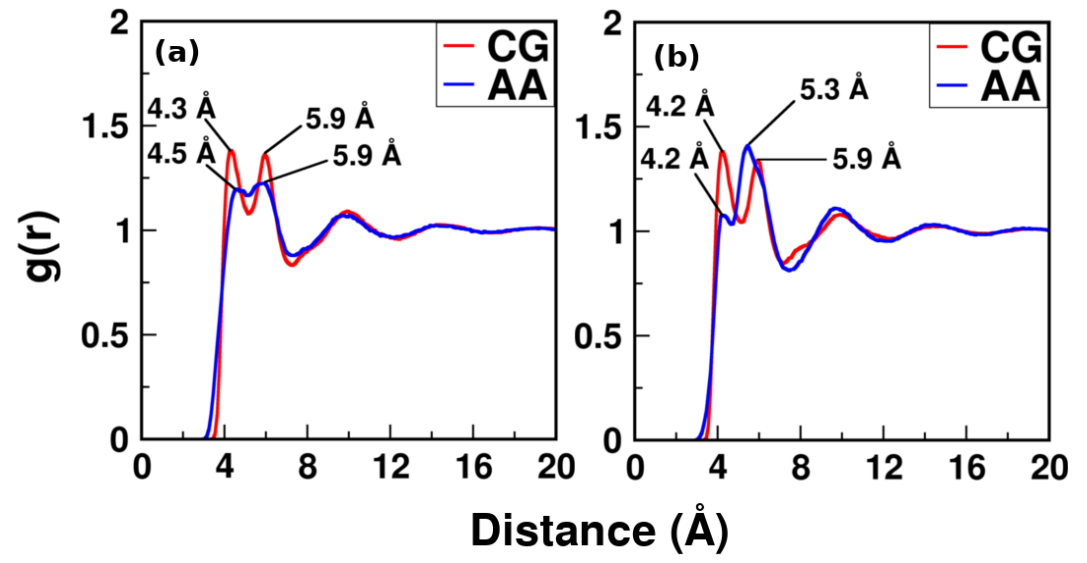

Figure C10.11: RDFs of (a) BZ and (b) NCR2

Analogue 2: Pyrrole

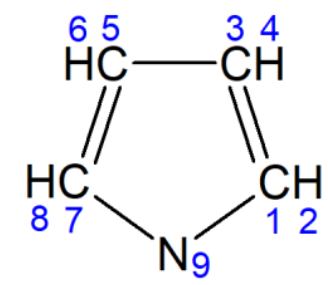

$\mathrm{H}_{10}$

Figure C10.12: All-atom pyrrole with atom numbering

\section{PL3}

NCR1

Figure C10.13: CG model of pyrrole showing bead types 


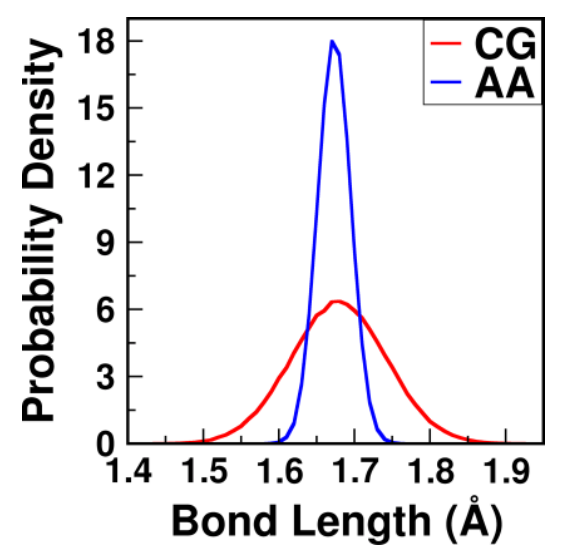

Figure C10.14: Distribution of bond NCR1-PL3

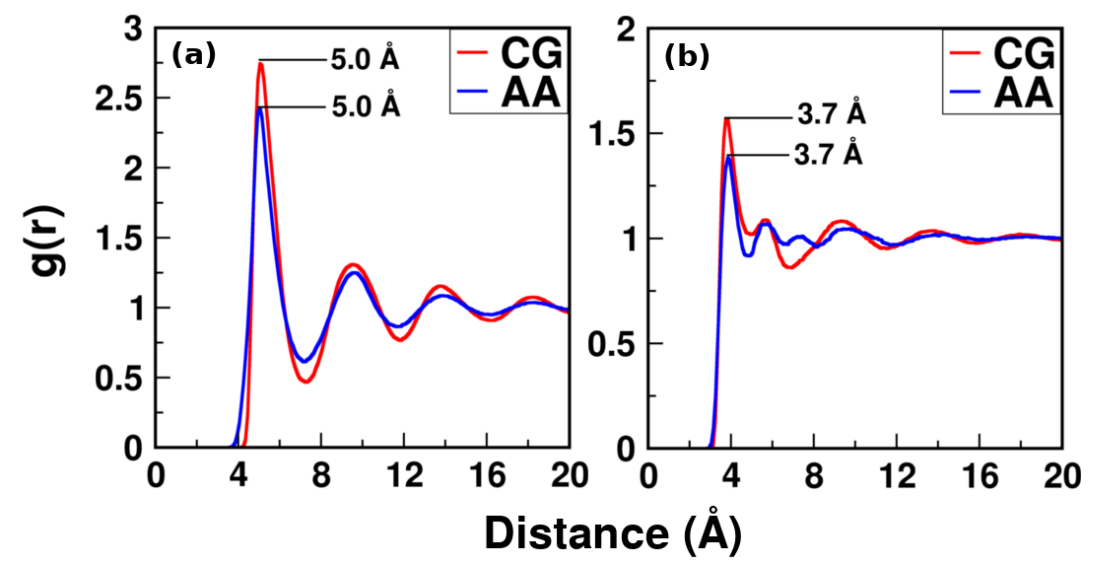

Figure C10.15: RDFs of (a) PL3 and (b) NCR1

Analogue 3: Toluene

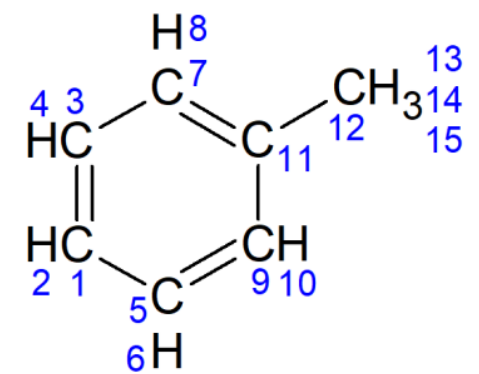

Figure C10.16: All-atom toluene with atom numbering

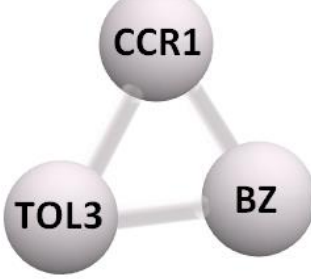

Figure C10.17: CG model of toluene showing bead types 

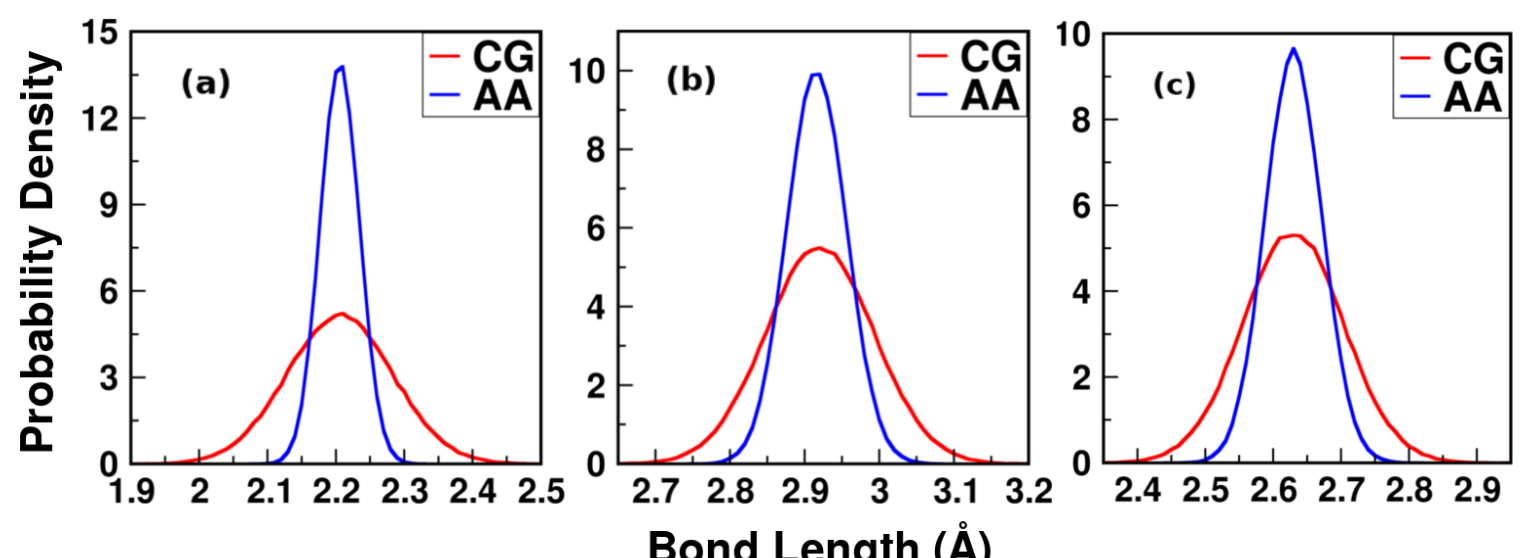

Figure C10.18: Distribution of bonds (a) TOL3-BZ (b) TOL3-CCR1, and (c) BZ-CCR1
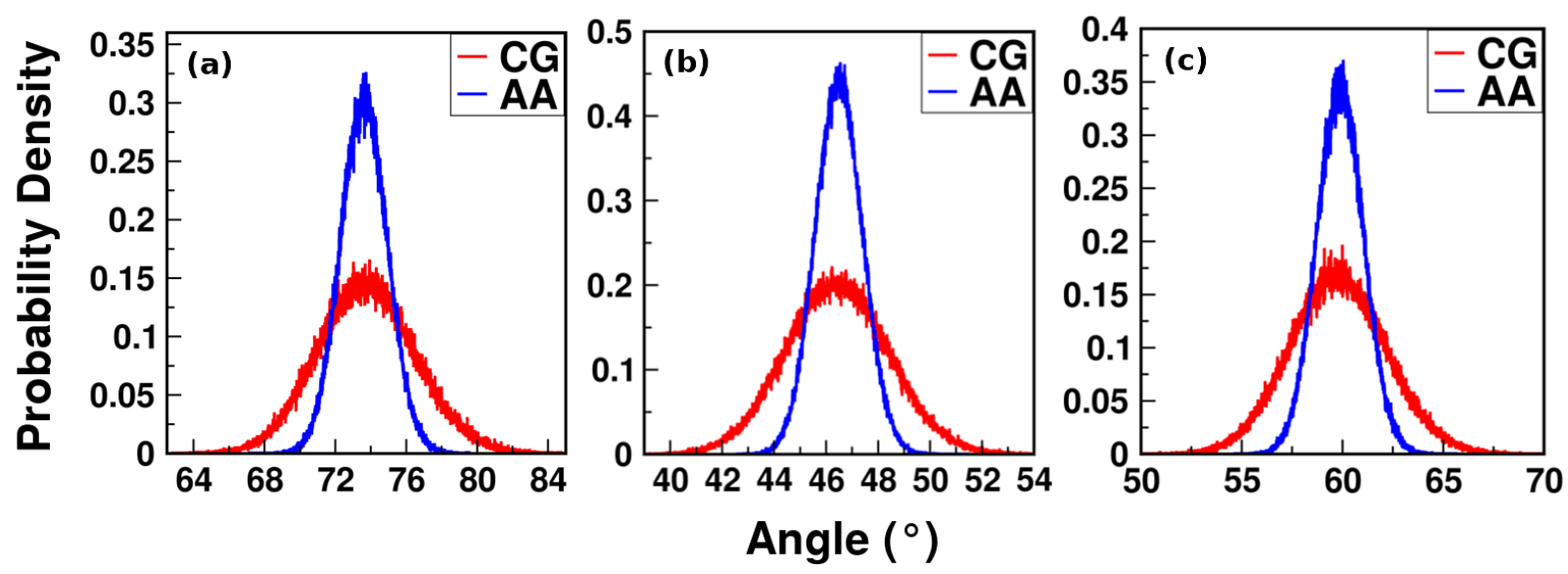

Figure C10.19: Distributions of angles (a) TOL3-BZ-CCR1, (b) TOL3-CCR1-BZ, and (c) BZ-TOL3-CCR1
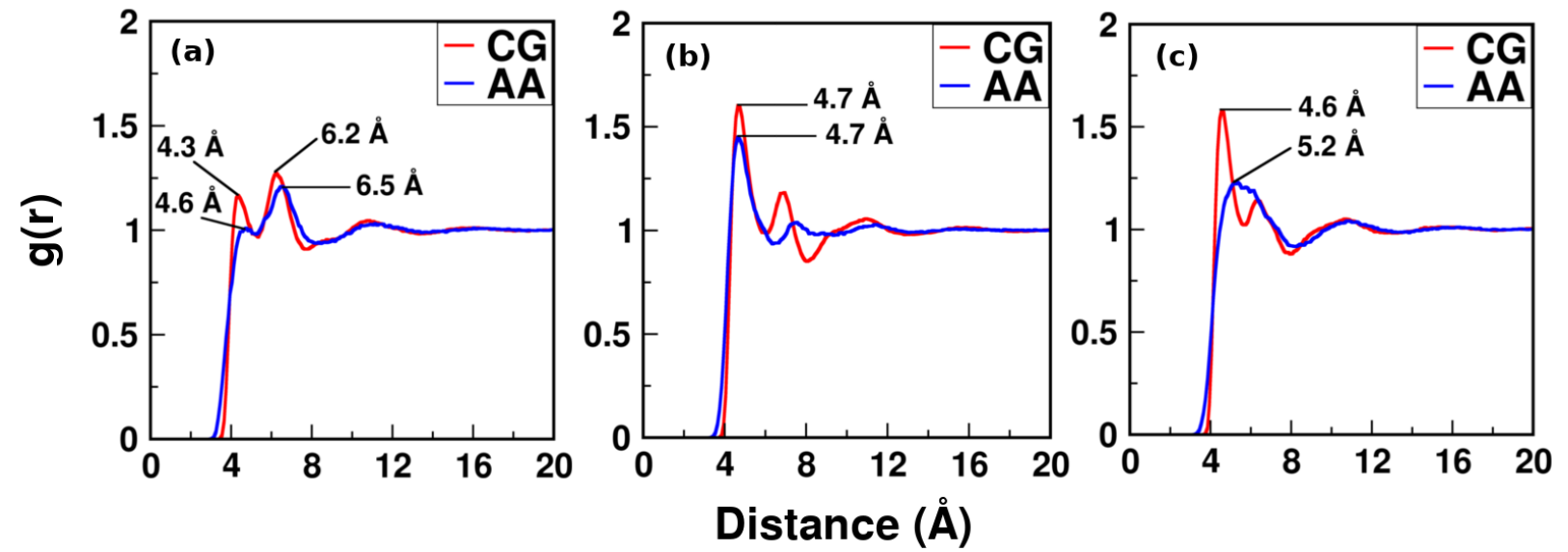

Figure C10.20: RDFs of (a) BZ, (b) CCR1, and (c) TOL3 


\section{C11: Isoleucine}

\section{Ile-Ile Dipeptide}

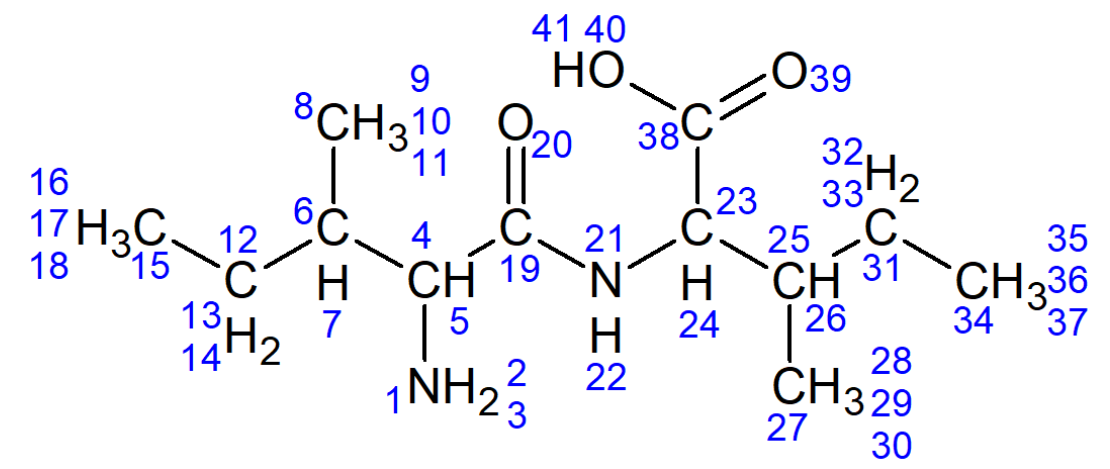

Figure C11.1: All-atom Isoleucine dipeptide with atom numbering

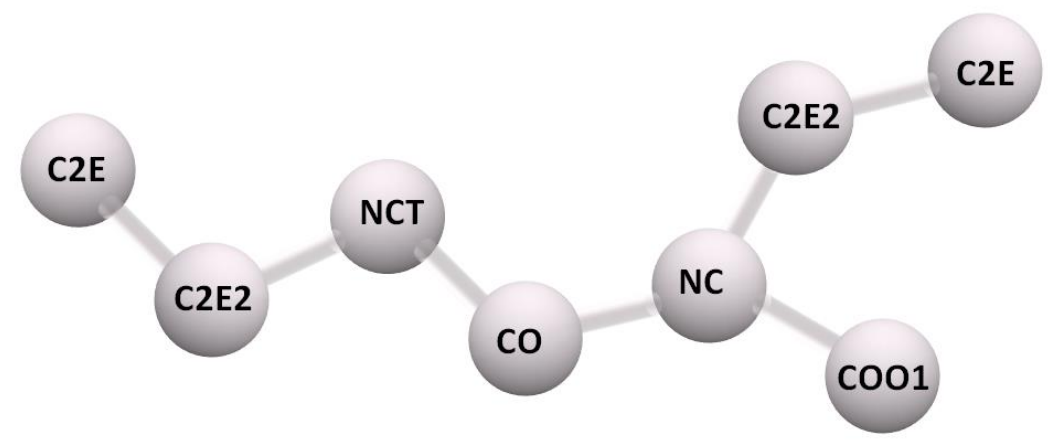

Figure C11.2: CG model of Isoleucine dipeptide showing bead types 

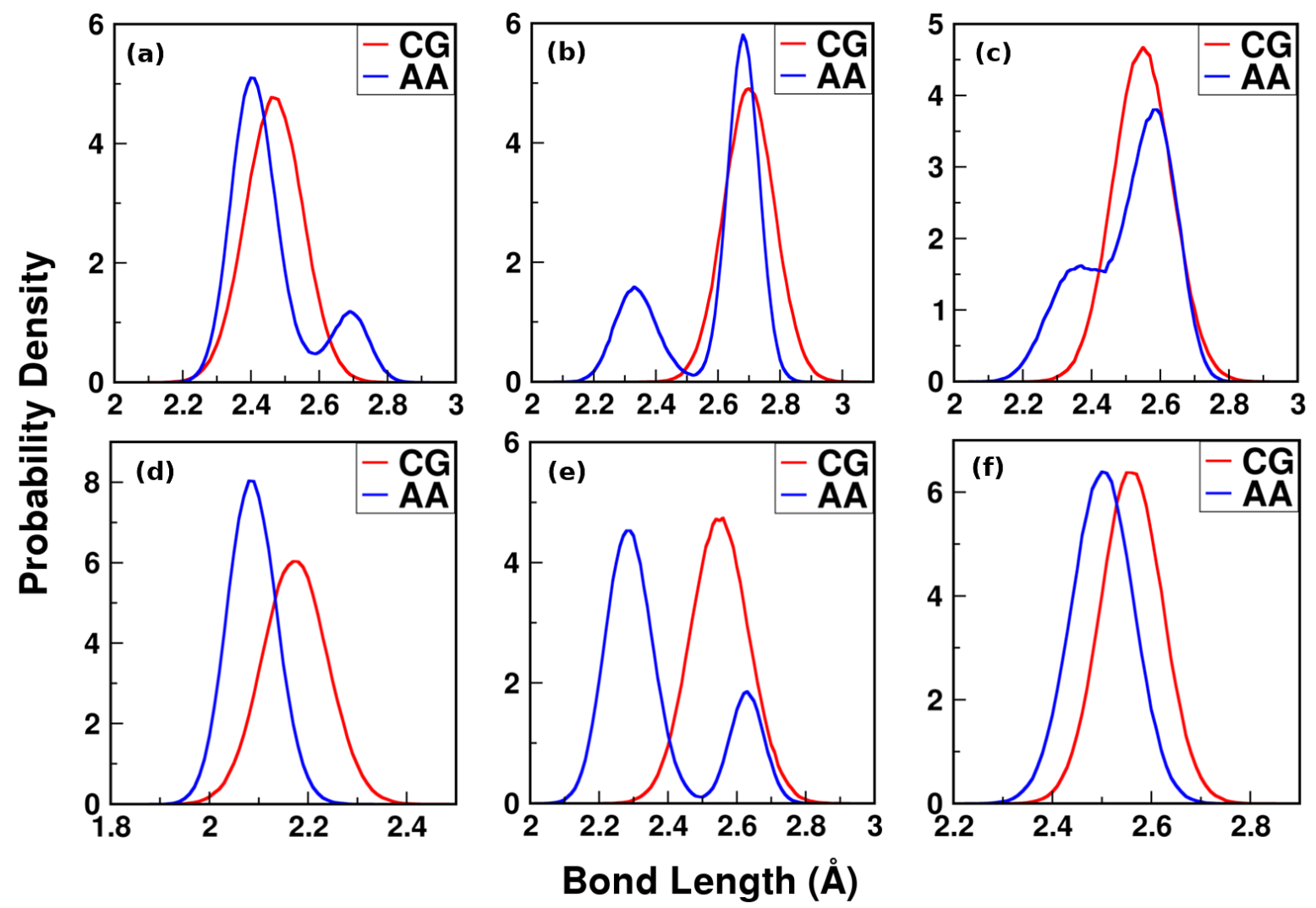

Figure C11.3: Distributions of bonds (a) C2E-C2E2, (b) C2E2-NCT, (c) NCT-CO, (d) CO-NC, (e) NC$\mathrm{C} 2 \mathrm{E} 2$, and (f) NC-COO1 

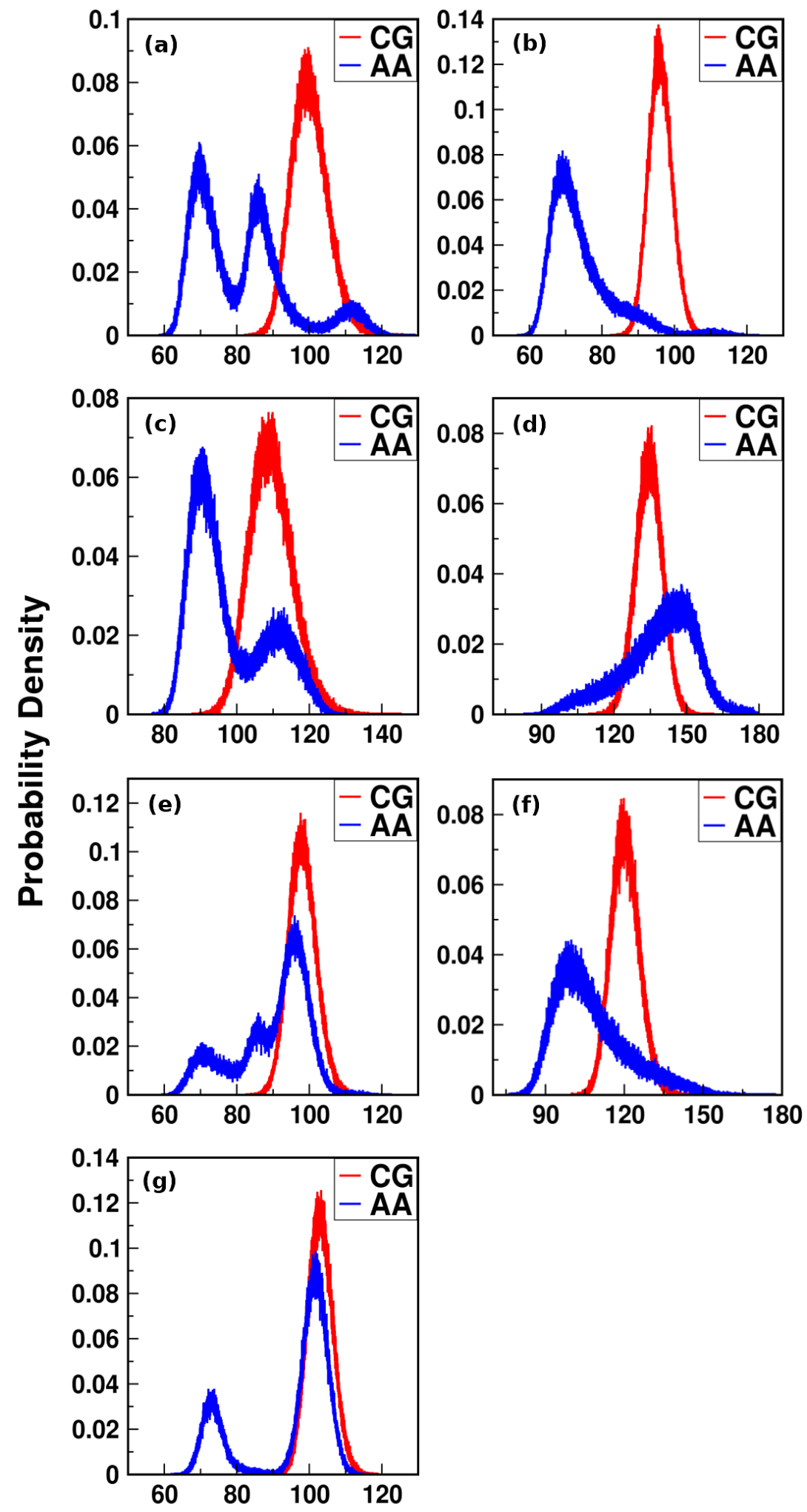

Angle $\left({ }^{\circ}\right)$

Figure C11.4: Distribution of angles (a) C2E-C2E2-NCT, (b) C2E2-NCT-CO, (c) NCT-CO-NC, (d) CONC-C2E2, (e) NC-C2E2-C2E, (f) CO-NC-COO1, and (g) C2E2-NC-COO1 

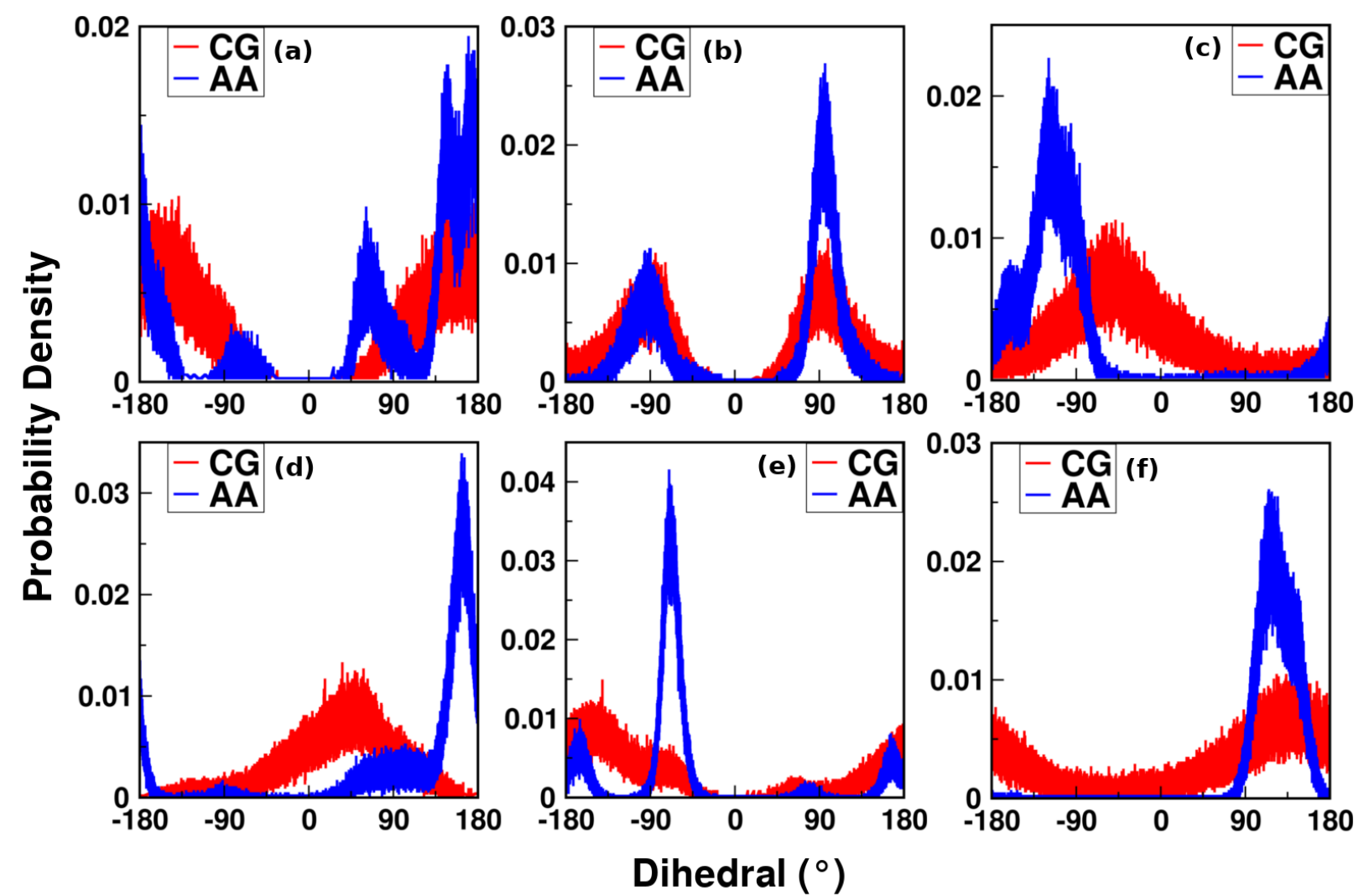

Figure C11.5: Distributions of dihedrals (a) C2E-C2E2-NCT-CO, (b) C2E2-NCT-CO-NC, (c) NCT-CONC-C2E2, (d) CO-NC-C2E2-C2E, (e) C2E-C2E2-NC-COO1, and (f) NCT-CO-NC-COO1

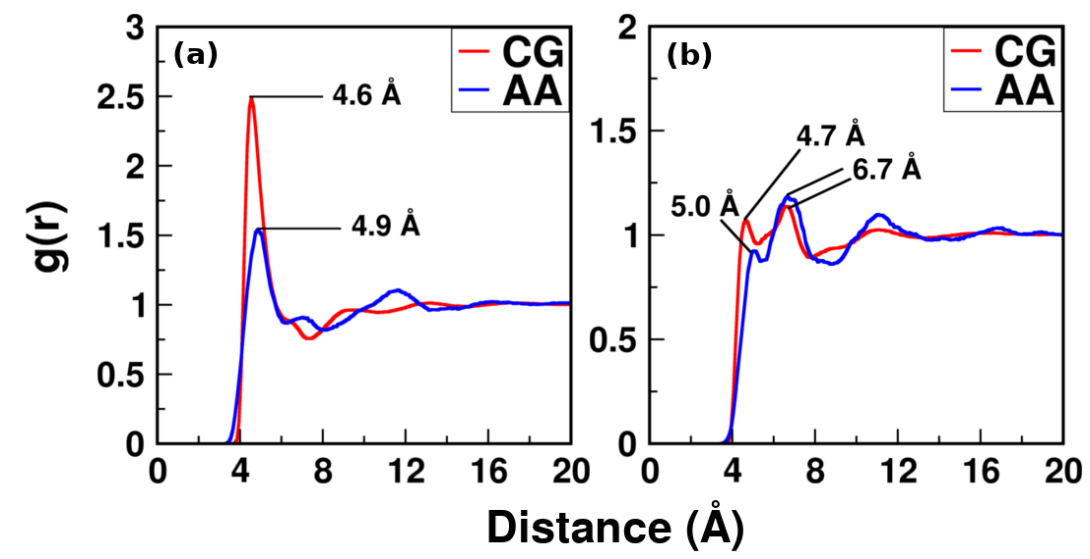

Figure C11.6: RDFs of (a) C2E and (b) C2E2 
Analogue: 3,4-dimethylhexane

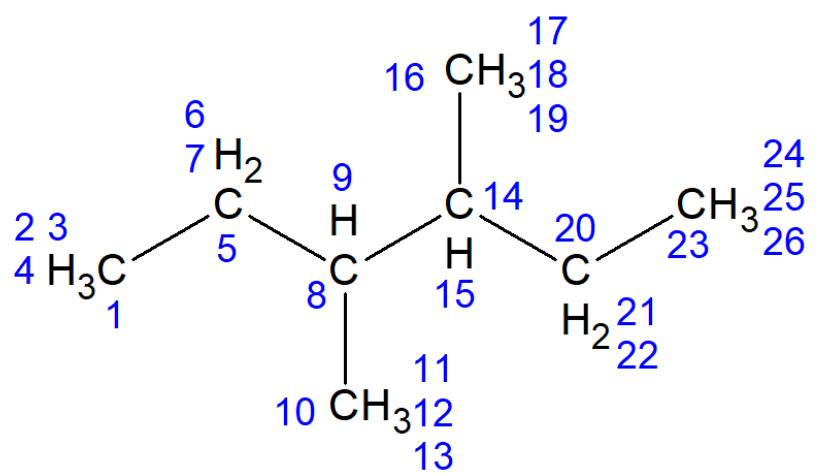

Figure C11.7: All-atom 3,4-dimethylhexane with atom numbering

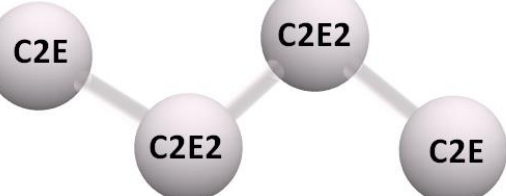

Figure C11.8: CG model of 3,4-dimethylhexane showing bead types

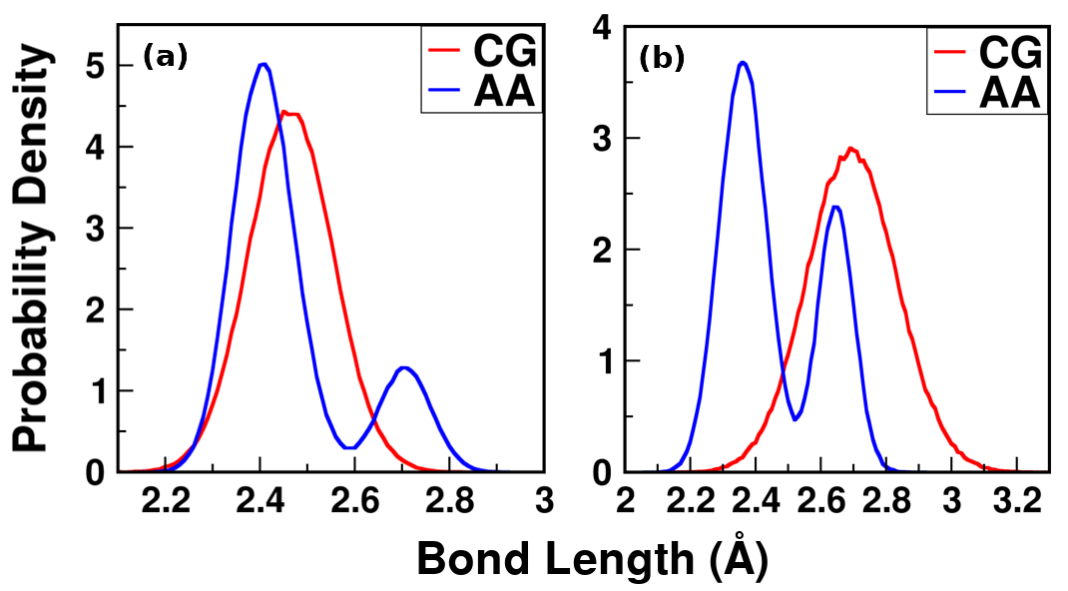

Figure C11.9: Distributions of bonds (a) C2E-C2E2 and (b) C2E2-C2E2

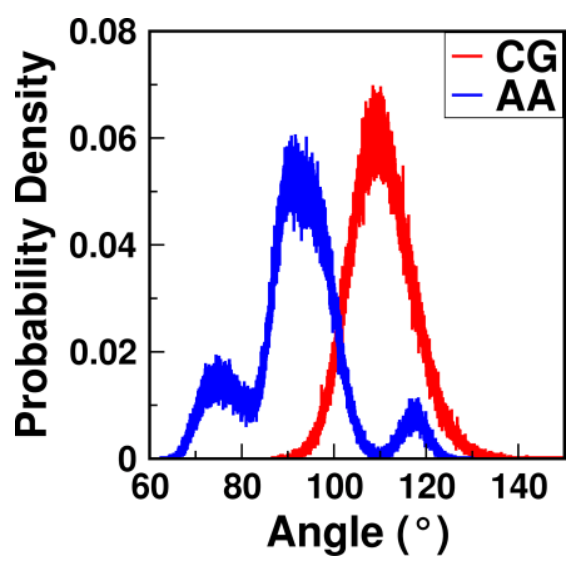

Figure C11.10: Distribution of angle C2E-C2E2-C2E2 


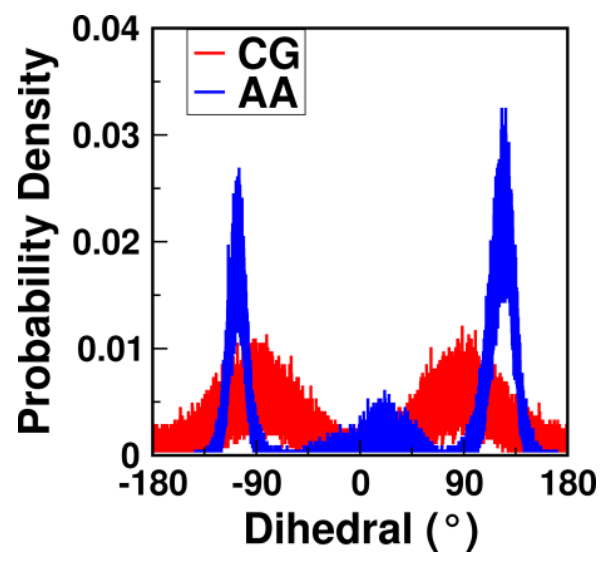

Figure C11.11: Distribution of dihedral C2E-C2E2-C2E2-C2E

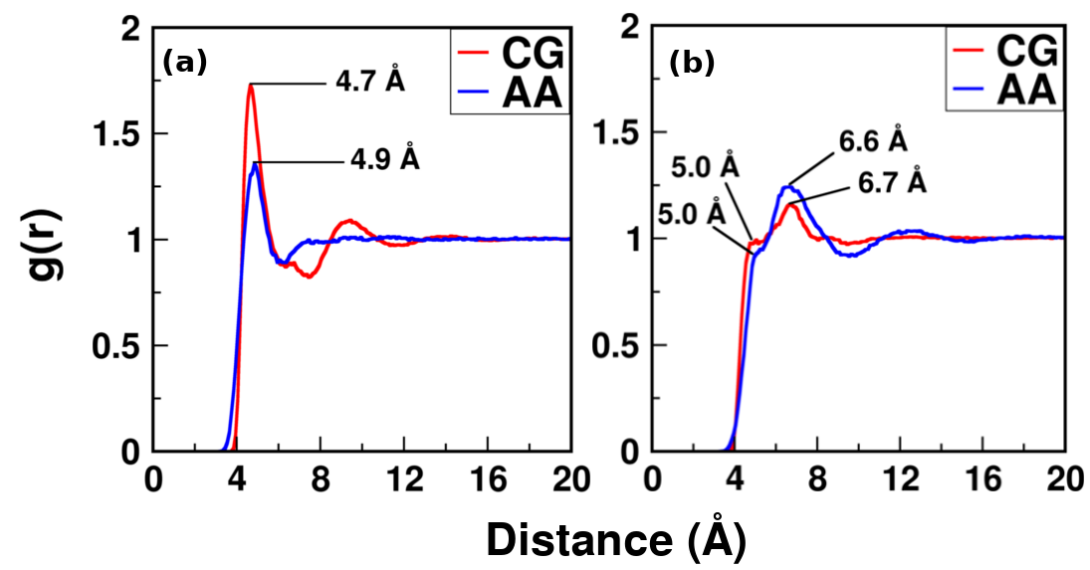

Figure C11.12: RDFs of (a) C2E and (b) C2E2 


\section{C12: Leucine}

\section{Leu-Leu Dipeptide}

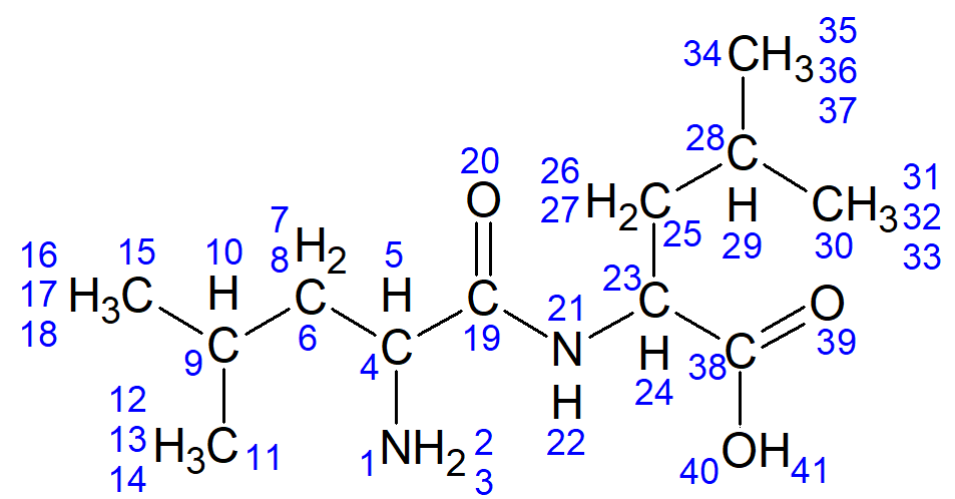

Figure C12.1: All-atom Leucine dipeptide with atom numbering

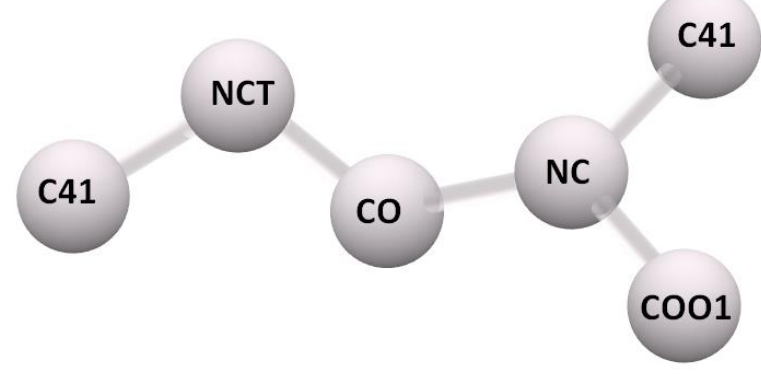

Figure C12.2: CG model of Leucine dipeptide showing bead types 

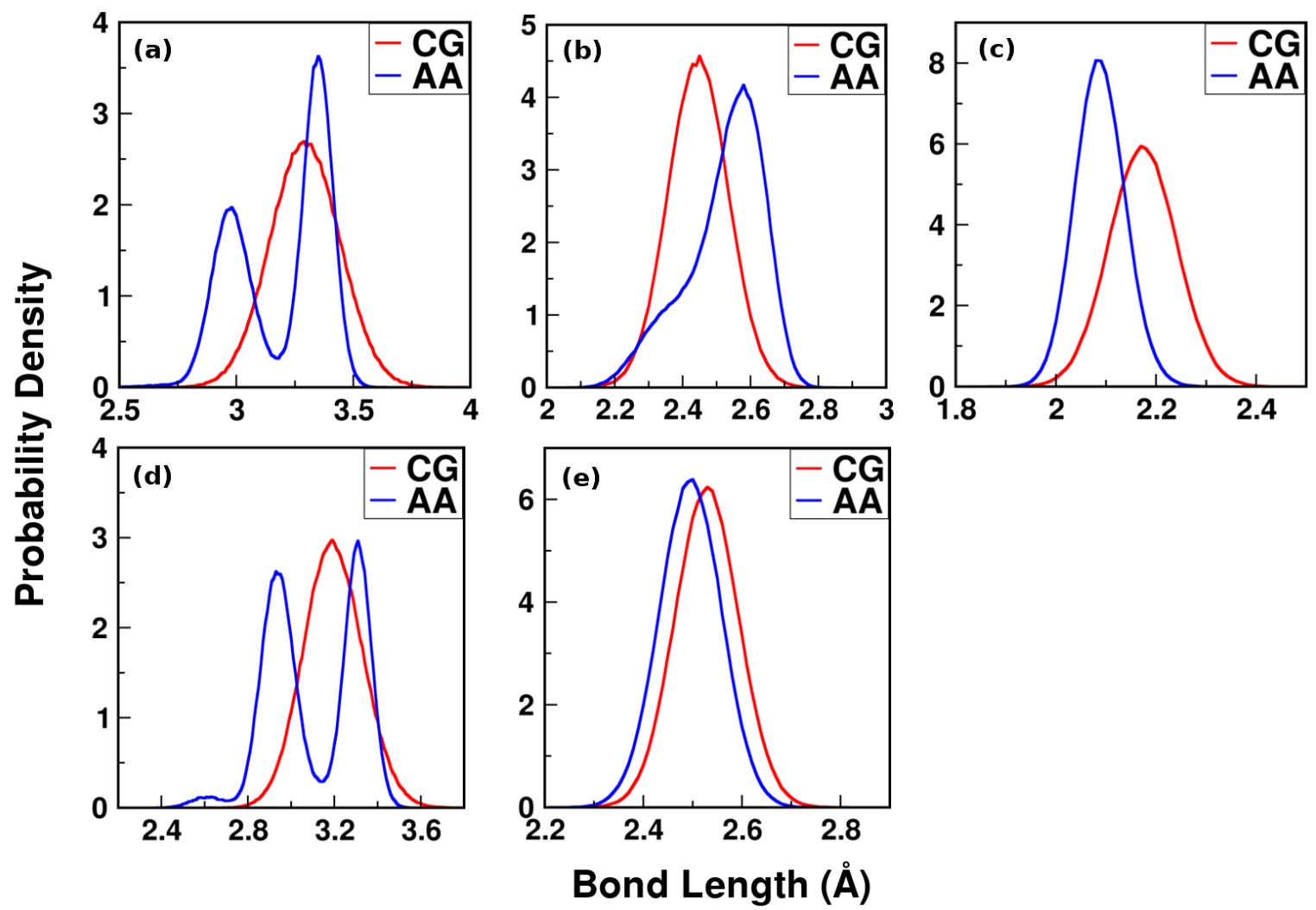

Figure C12.3: Distribution of bonds (a) C41-NCT, (b) NCT-CO, (c) CO-NC, (d) NC-C41, and (e) NCCOO1
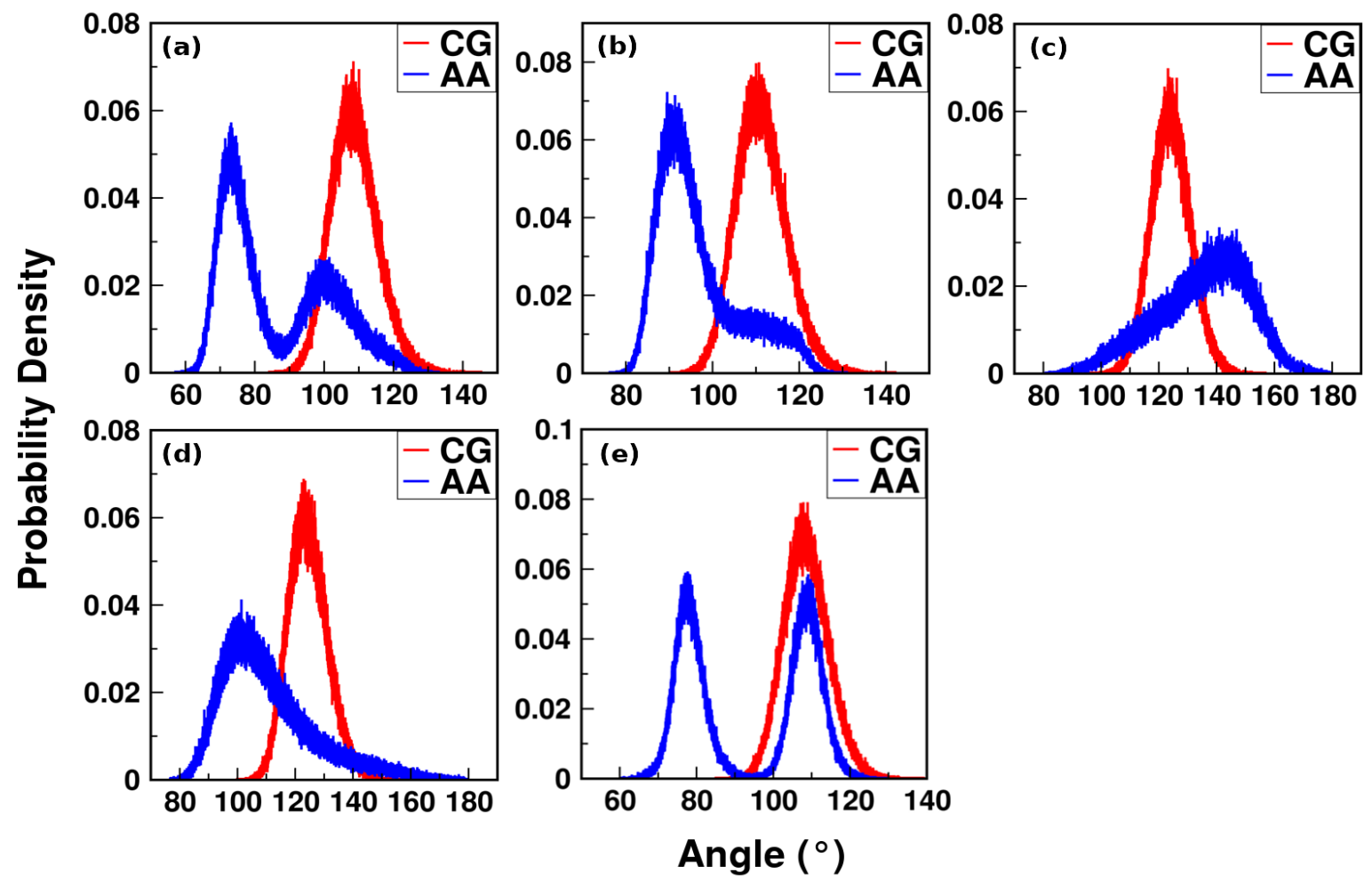

Figure C12.4: Distribution of angles (a) C41-NCT-CO, (b) NCT-CO-NC, (c) CO-NC-C41, (d) CO-NCCOO1, and (e) C41-NC-COO1 

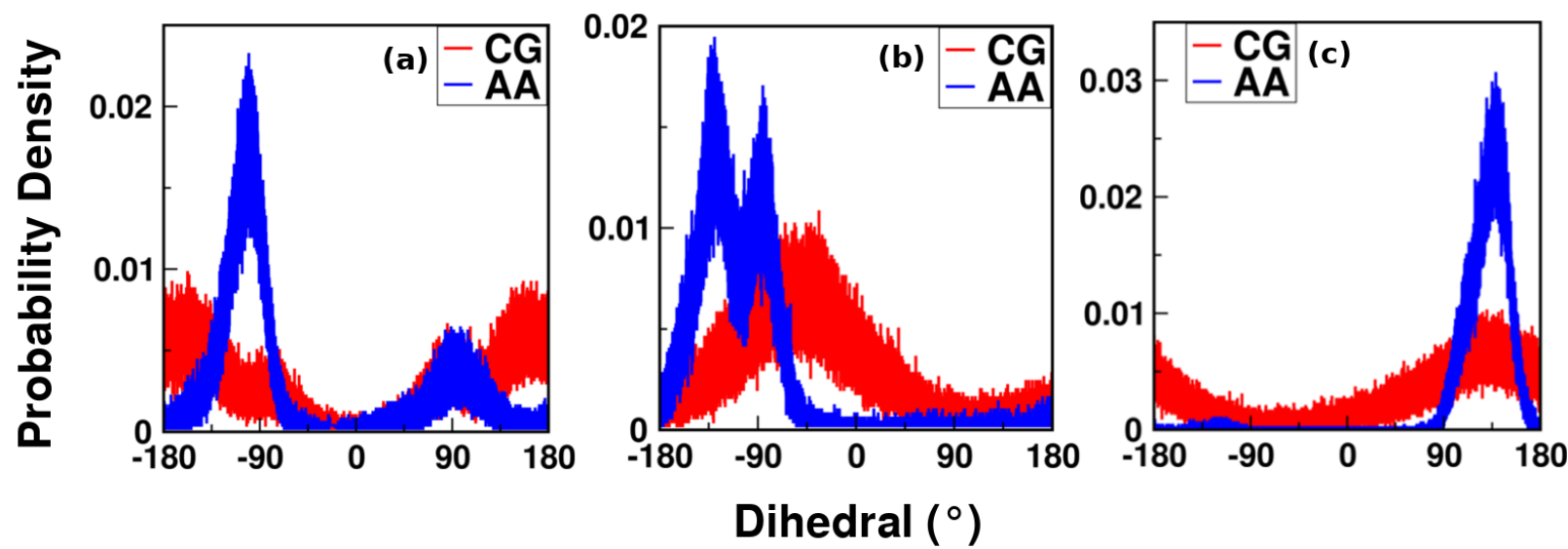

Figure C12.5: Distribution of dihedrals (a) C41-NCT-CO-NC, (b) NCT-CO-NC-C41, and (c) NCT-CO-NCCOO1

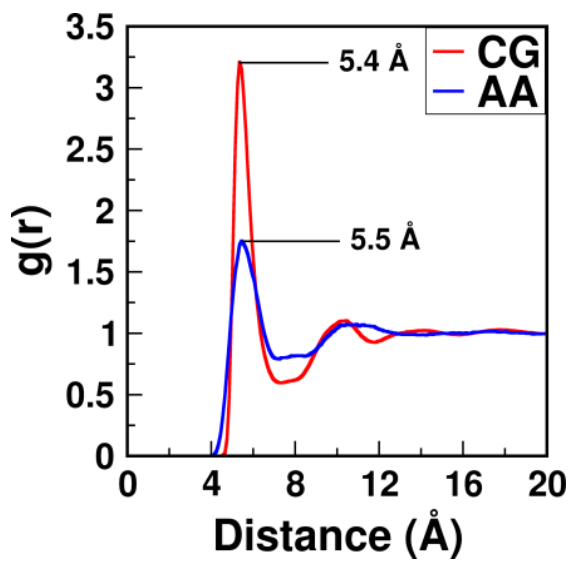

Figure C12.6: RDF of C41

Analogue: 2,5-dimethylhexane

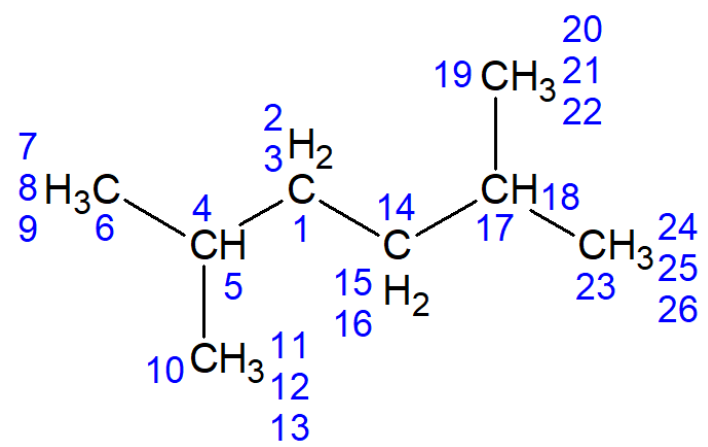

Figure C12.7: All-atom 2,5-dimethylhexane with atom numbering

\section{C41}

Figure C12.8: CG model of 2,5-dimethylhexane showing bead types 


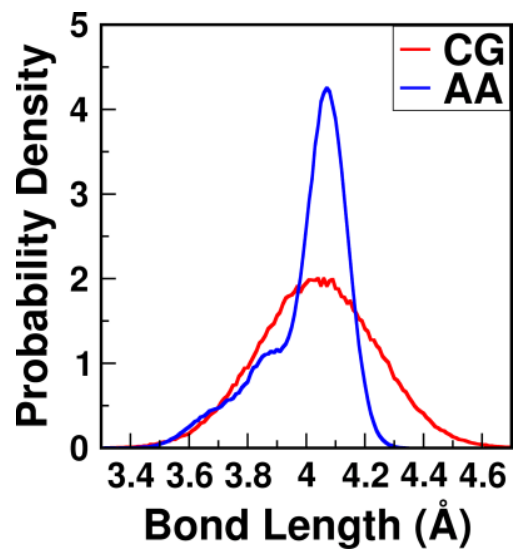

Figure C12.9: Distribution of bond C41-C41

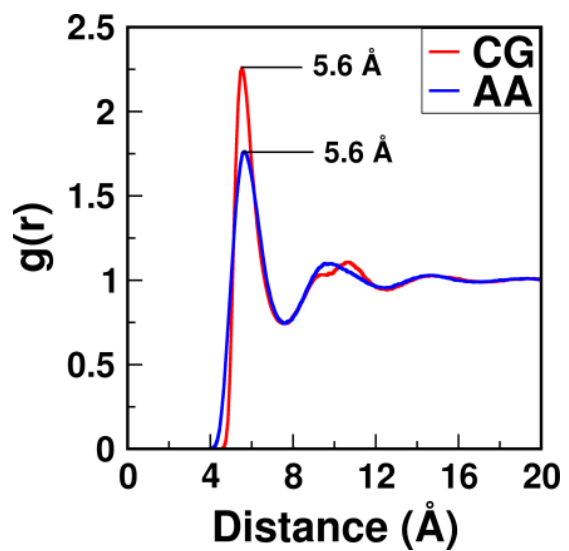

Figure C12.10: RDF of C41 


\section{C13: Lysine}

\section{Lys-Lys Dipeptide}

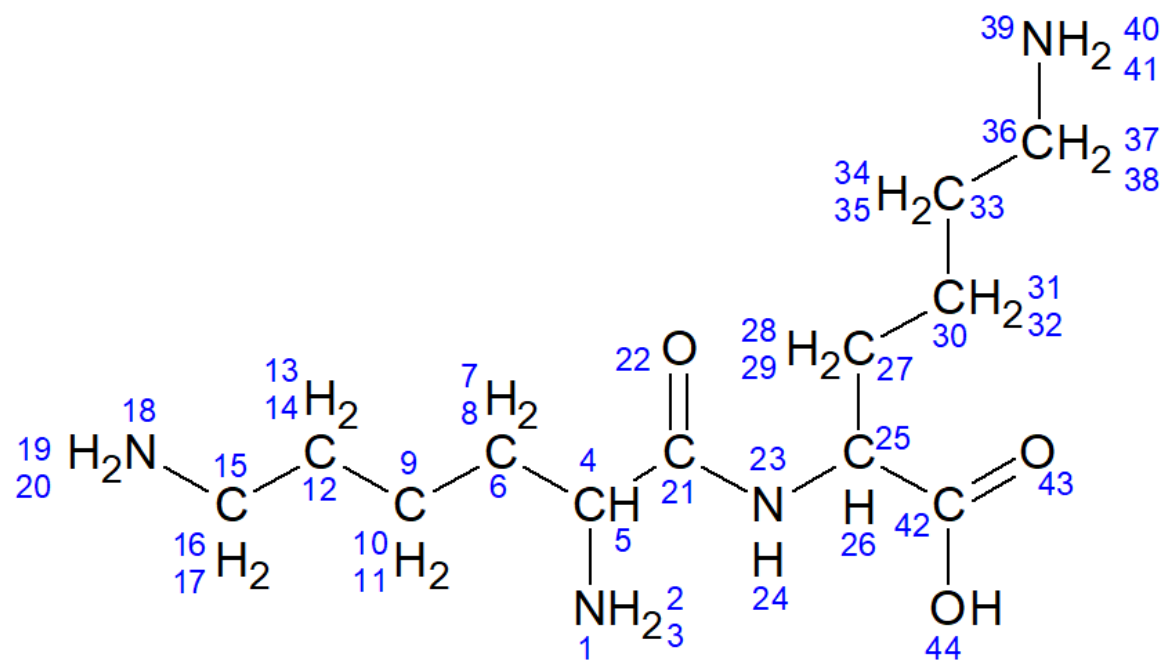

Figure C13.1: All-atom Lysine dipeptide with atom numbering

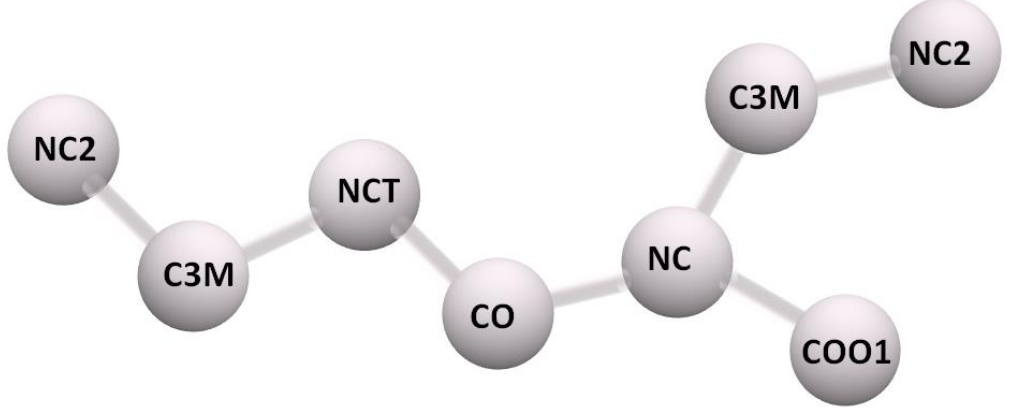

Figure C13.2: CG model of Lysine dipeptide 

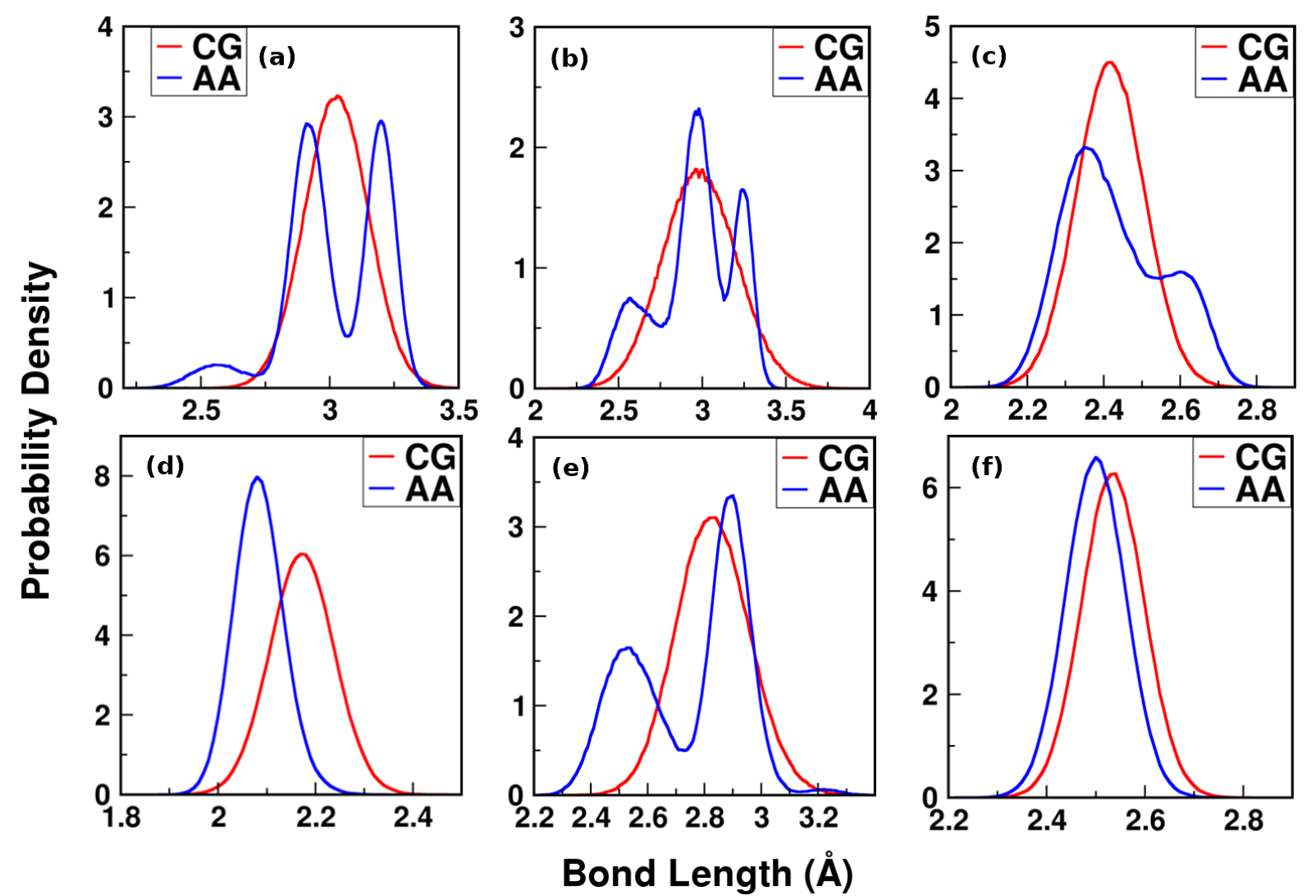

Figure C13.3: Distributions of bonds (a) NC2-C3M, (b) C3M-NCT, (c) NCT-CO, (d) CO-NC, (e) NC-C3M, and (f) NC-COO1 


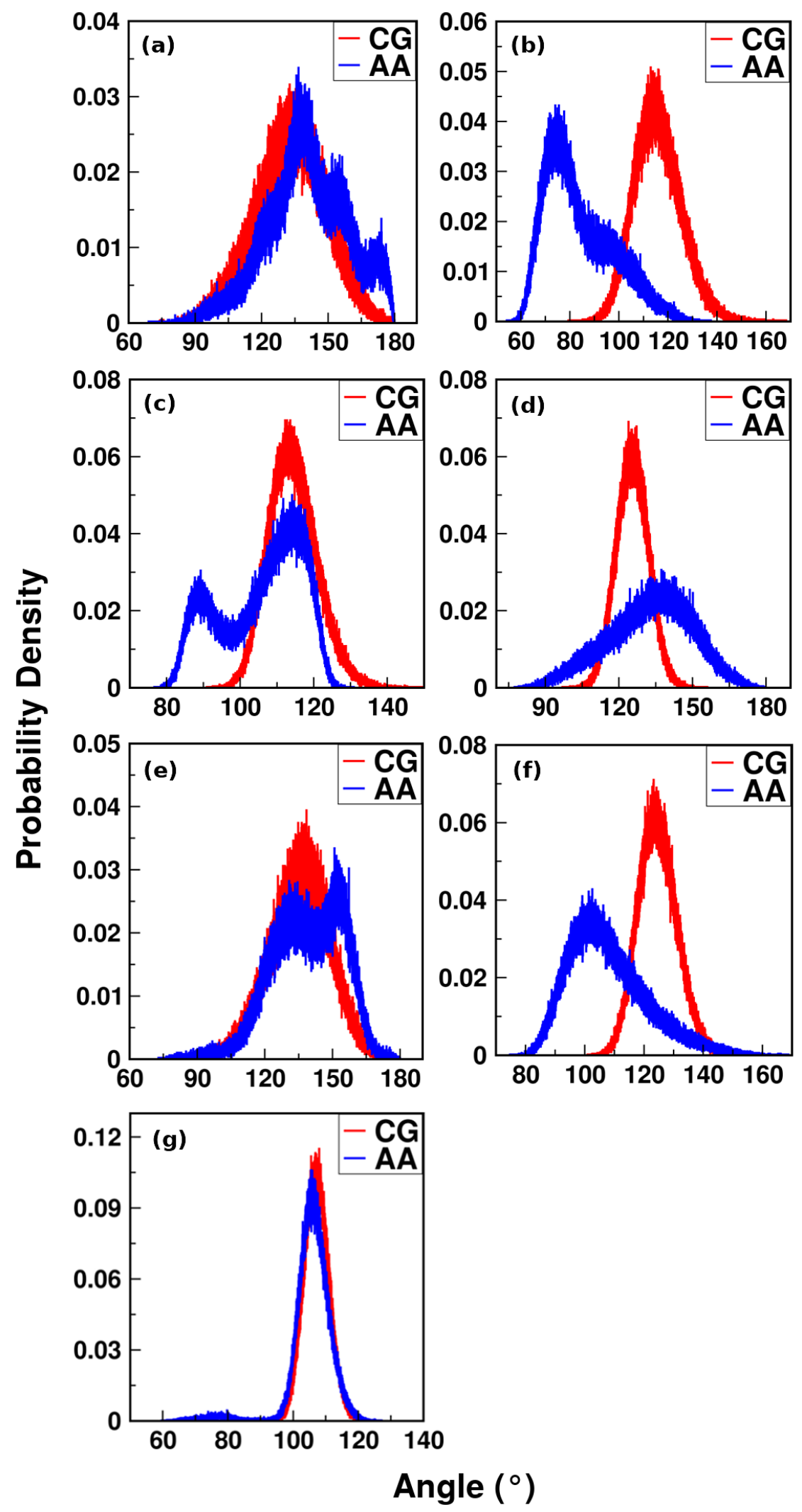

Figure C13.4: Distributions of angles (a) NC2-C3M-NCT, (b) C3M-NCT-CO, (c) NCT-CO-NC, (d) CO-NCC3M, (e) NC-C3M-NC2, (f) CO-NC-COO1, and (g) C3M-NC-COO1 

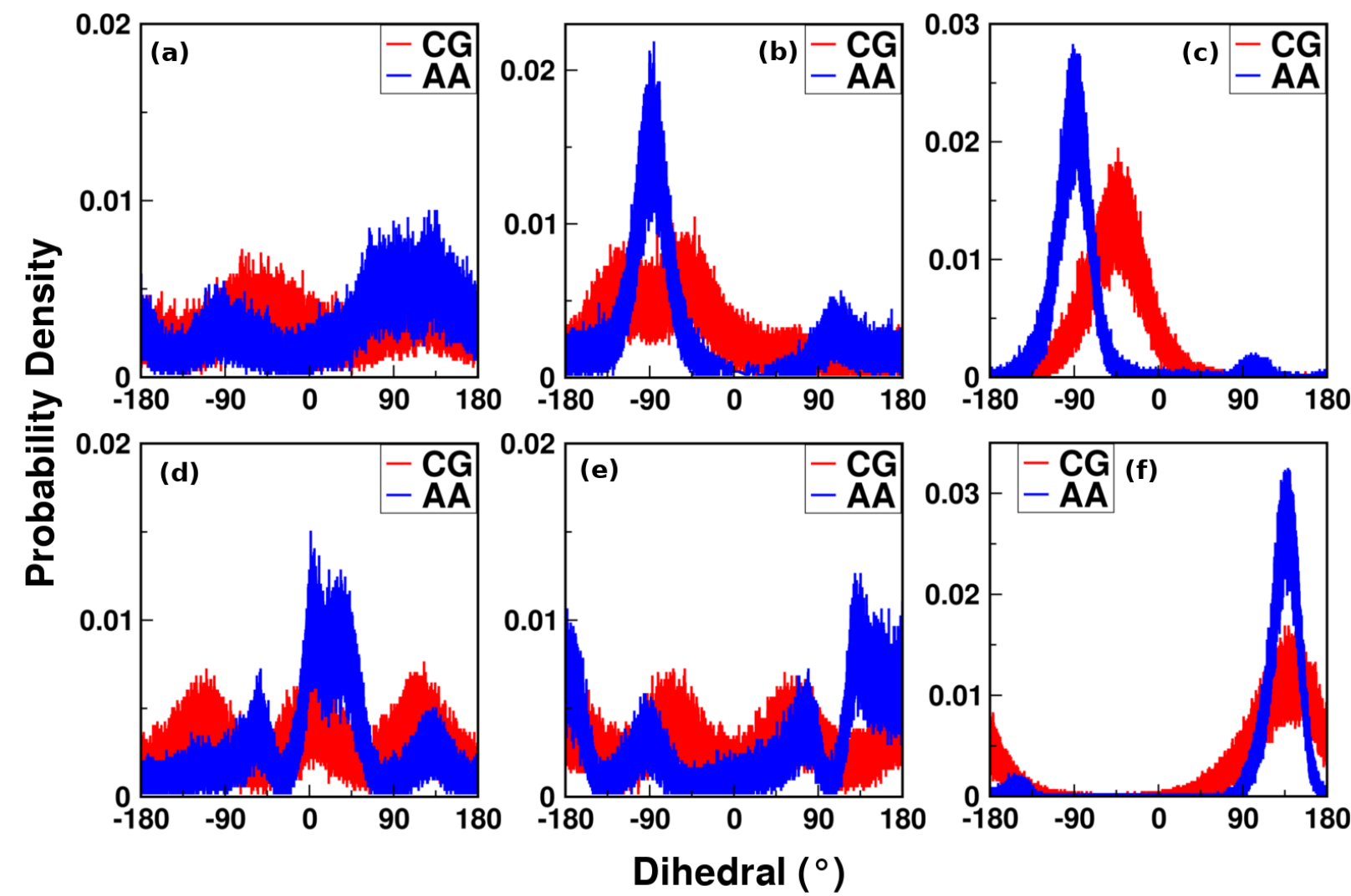

Figure C13.5: Distributions of dihedrals (a) NC2-C3M-NCT-CO, (b) C3M-NCT-CO-NC, (c) NCT-CO-NCC3M, (d) CO-NC-C3M-NC2, (e) NC2-C3M-NC-COO1, and (f) NCT-CO-NC-COO1

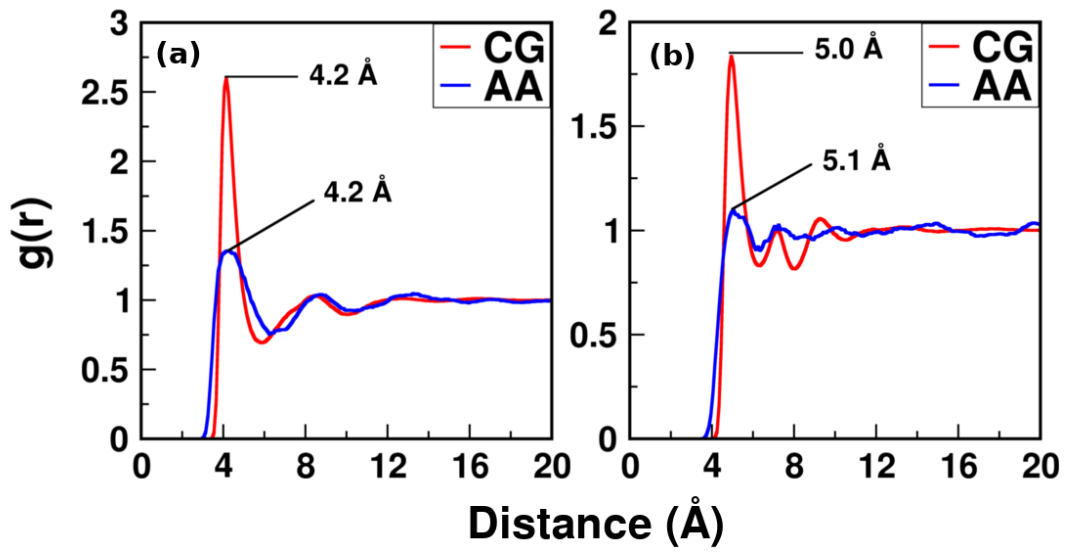

Figure C13.6: RDFs of (a) NC2 and (b) C3M

Analogue: N-butylamine

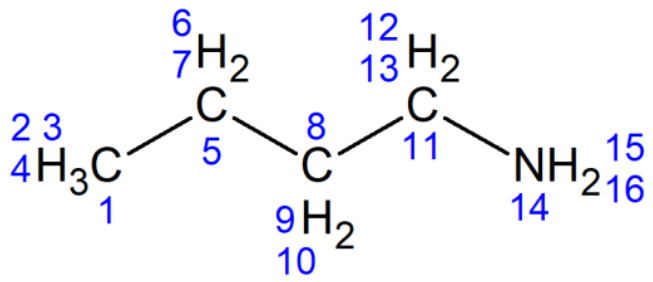

Figure C13.7: All-atom N-butylamine with atom numbering 


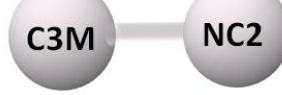

Figure C13.8: CG model of $\mathrm{N}$-butylamine showing bead types

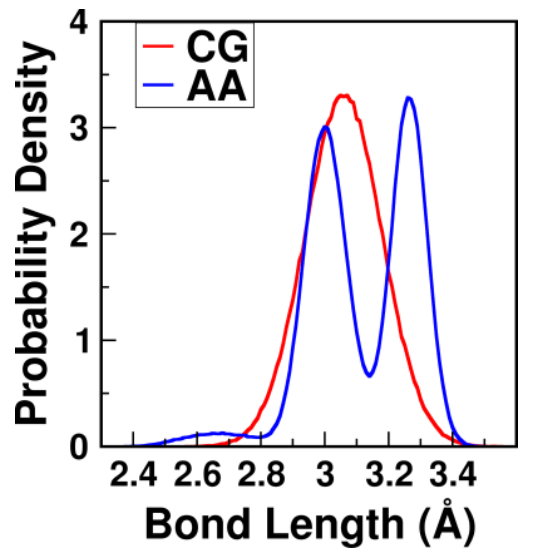

Figure C13.9: Distribution of bond NC2-C3M

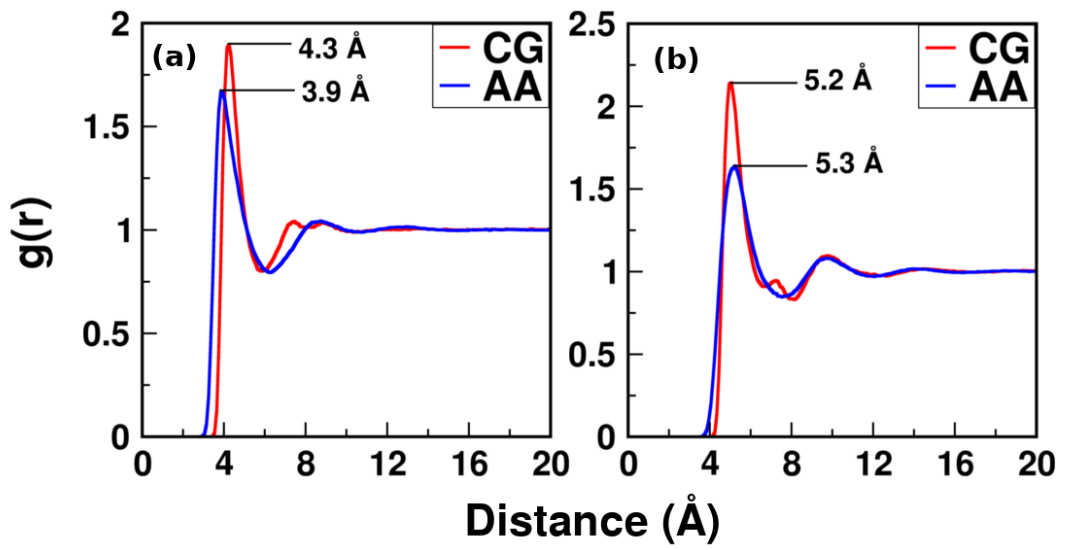

Figure C13.10: RDFs of (a) NC2 and (b) C3M 


\section{C14: Methionine}

Met-Met Dipeptide

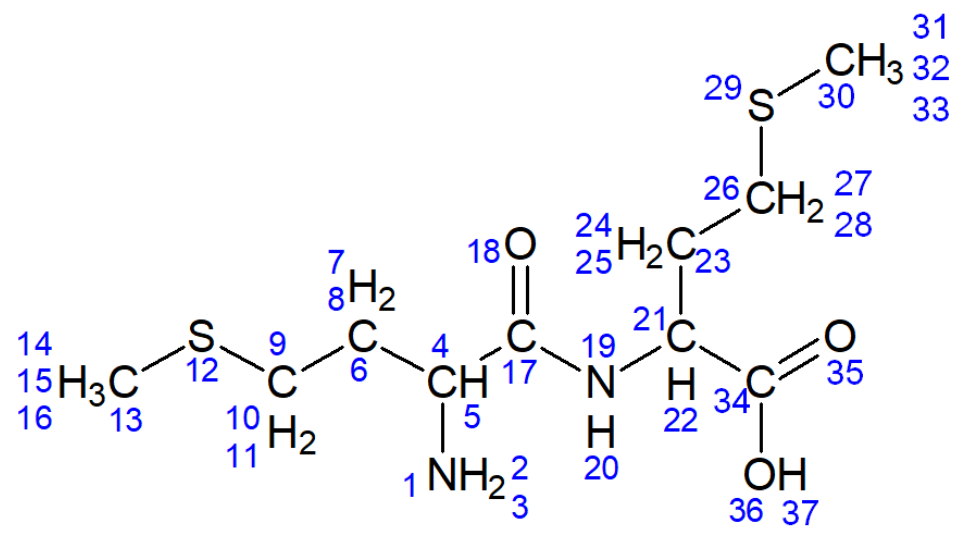

Figure C14.1: All-atom Methionine dipeptide with atom numbering

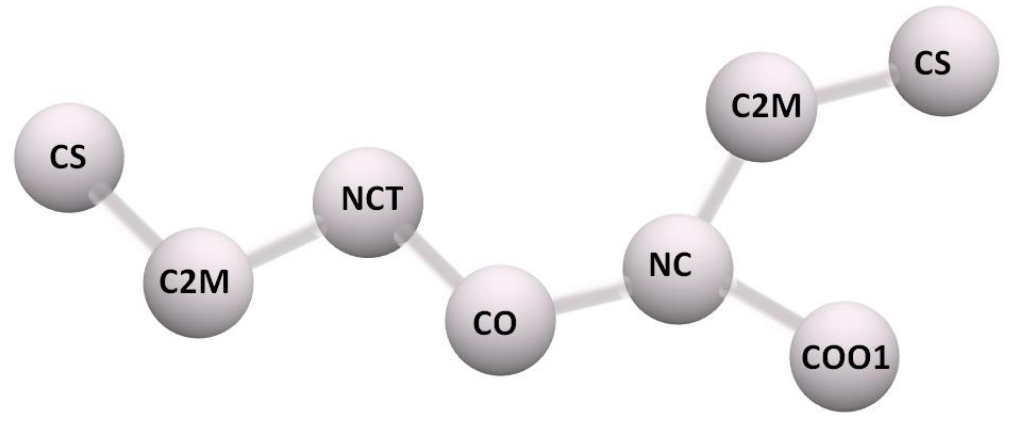

Figure C14.2: CG model of Methionine dipeptide showing bead types 

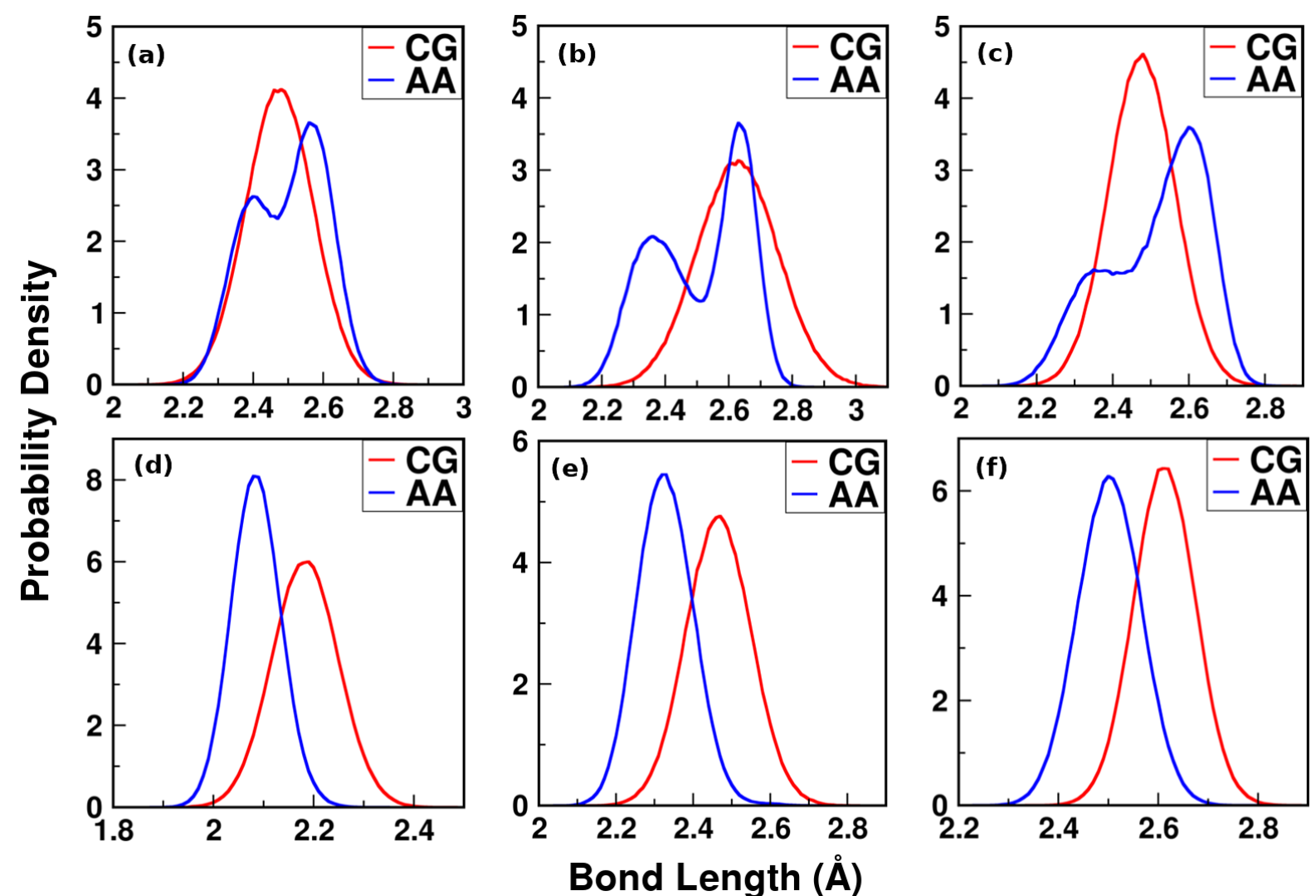

Figure C14.3: Distributions of bonds (a) CS-C2M, (b) C2M-NCT, (c) NCT-CO, (d) CO-NC, (e) NC-C2M, and (f) NC-COO1 

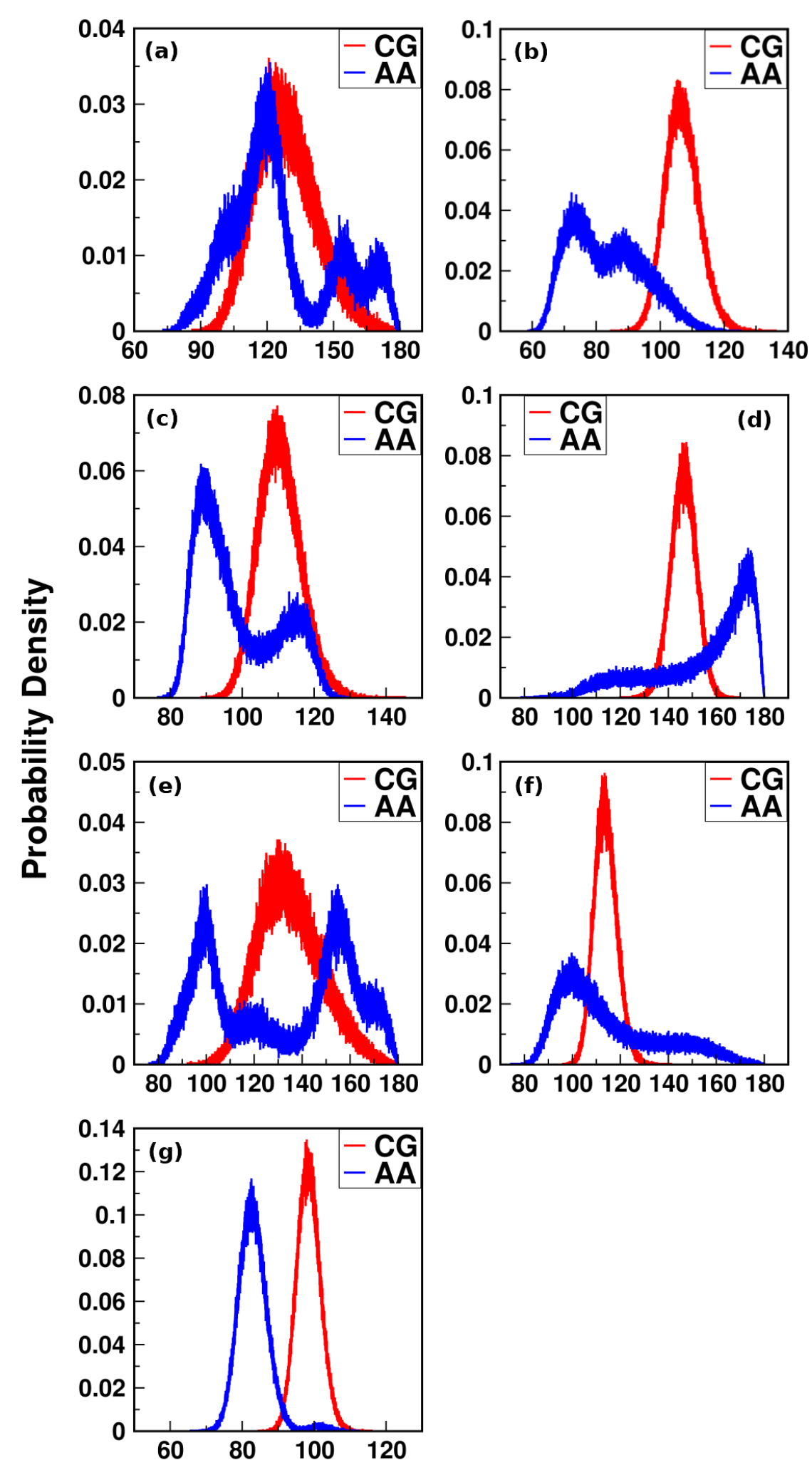

Angle $\left({ }^{\circ}\right)$

Figure C14.4: Distributions of angles (a) CS-C2M-NCT, (b) C2M-NCT-CO, (c) NCT-CO-NC, (d) CO-NCC2M, (e) NC-C2M-CS, (f) CO-NC-COO1, and (g) C2M-NC-COO1 

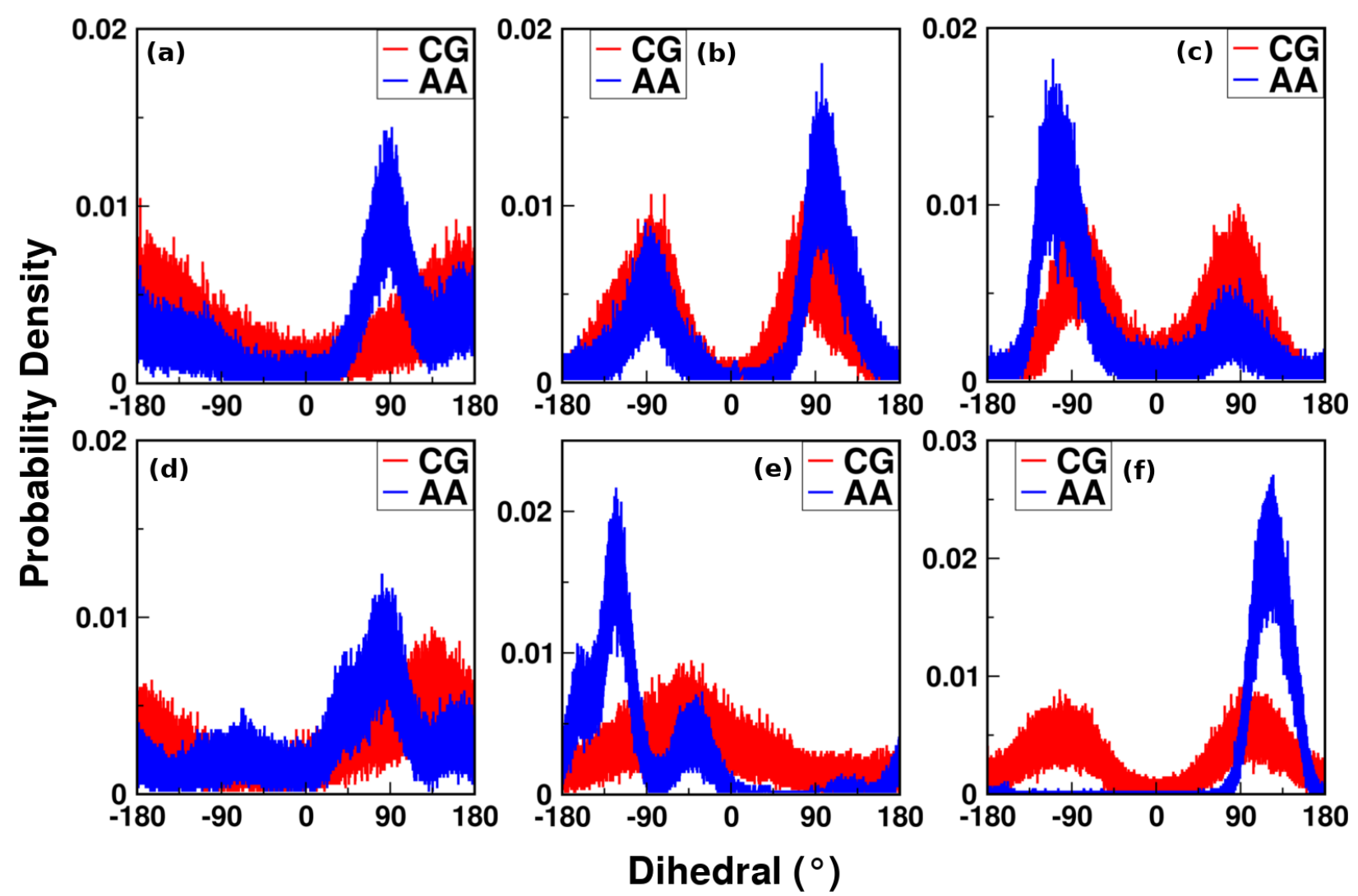

Figure C14.5: Distributions of dihedrals (a) CS-C2M-NCT-CO, (b) C2M-NCT-CO-NC, (c) NCT-CO-NCC2M, (d) CO-NC-C2M-CS, (e) CS-C2M-NC-COO1, and (f) NCT-CO-NC-COO1

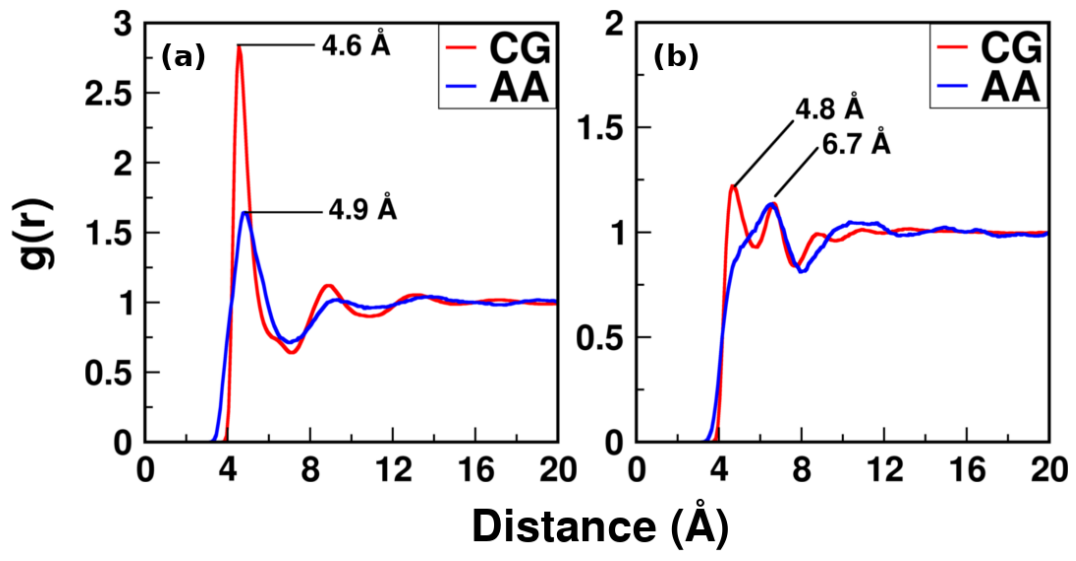

Figure C14.6: RDFs of (a) CS and (b) C2M

Analogue: Ethyl methyl sulfide

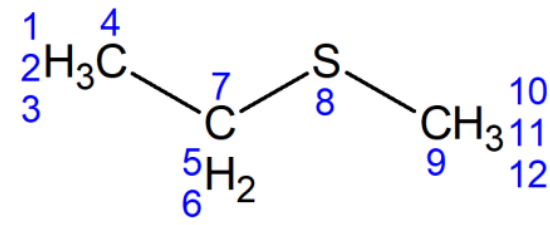

Figure C14.7: All-atom ethyl methyl sulfide with atom numbering 
Figure C14.8: CG model of ethyl methyl sulfide showing bead types

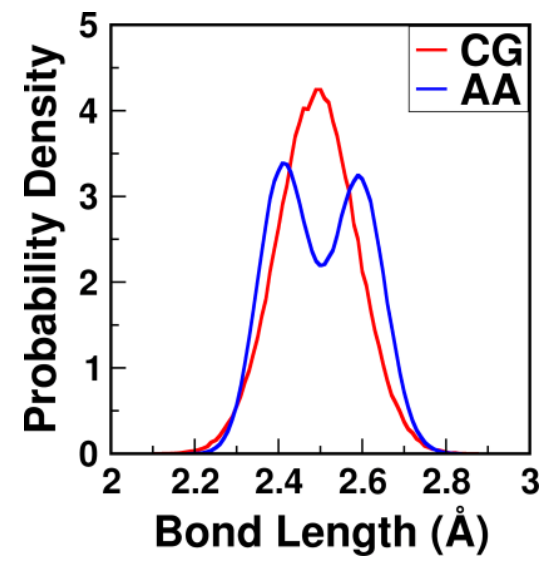

Figure C14.9: Distribution of bond CS-C2M

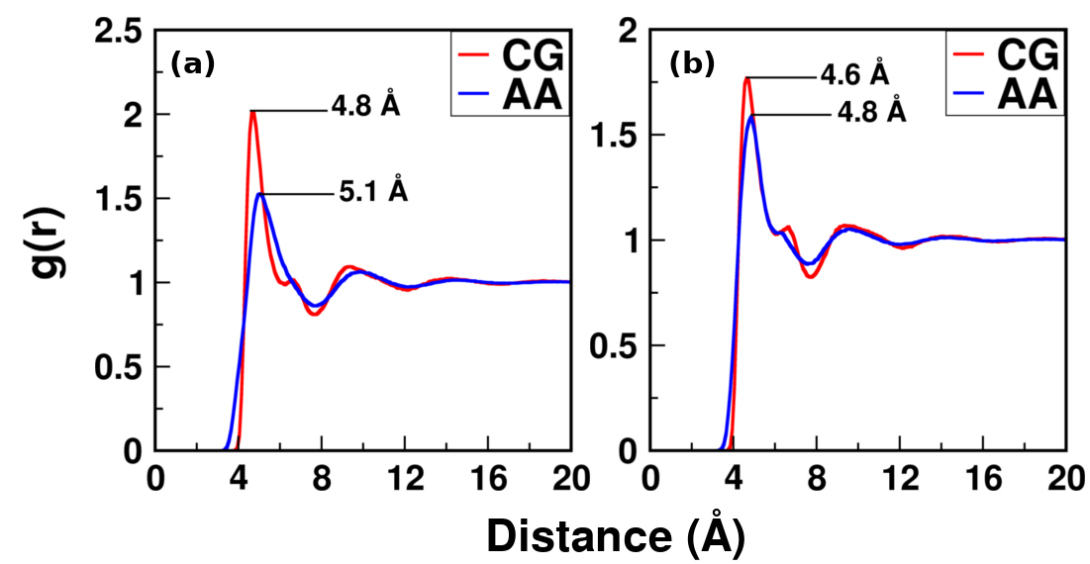

Figure C14.10: RDFs of (a) CS and (b) C2M 


\section{C15: Phenylalanine}

Phe-Phe Dipeptide

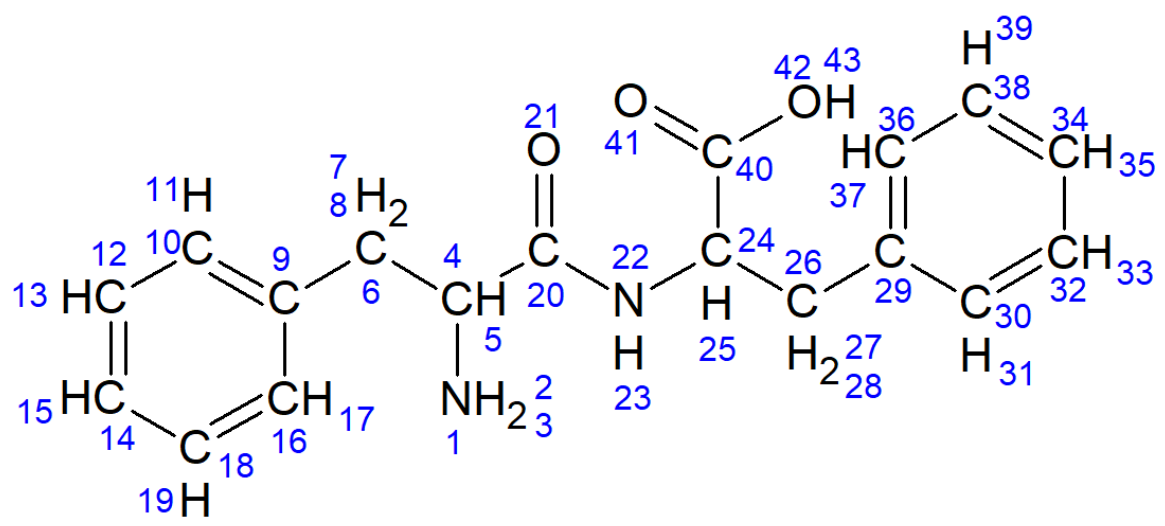

Figure C15.1: All-atom Phenylalanine dipeptide with atom numbering

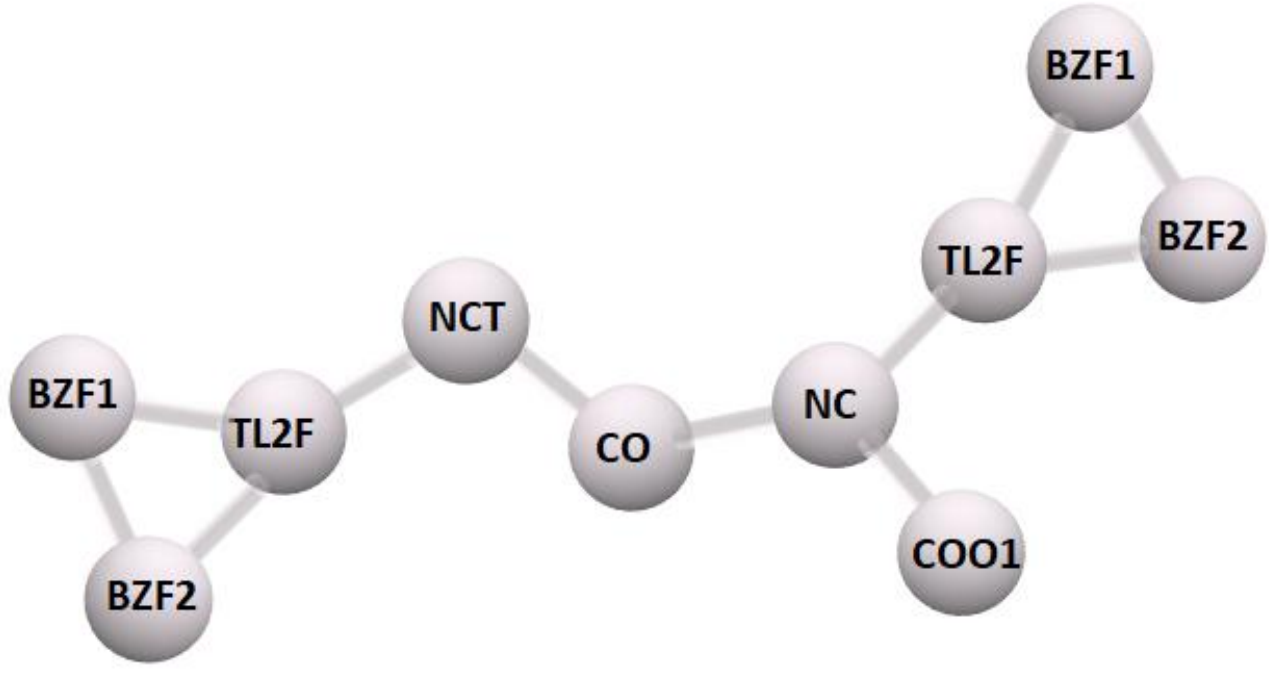

Figure C15.2: CG model of Phenylalanine dipeptide showing bead types 


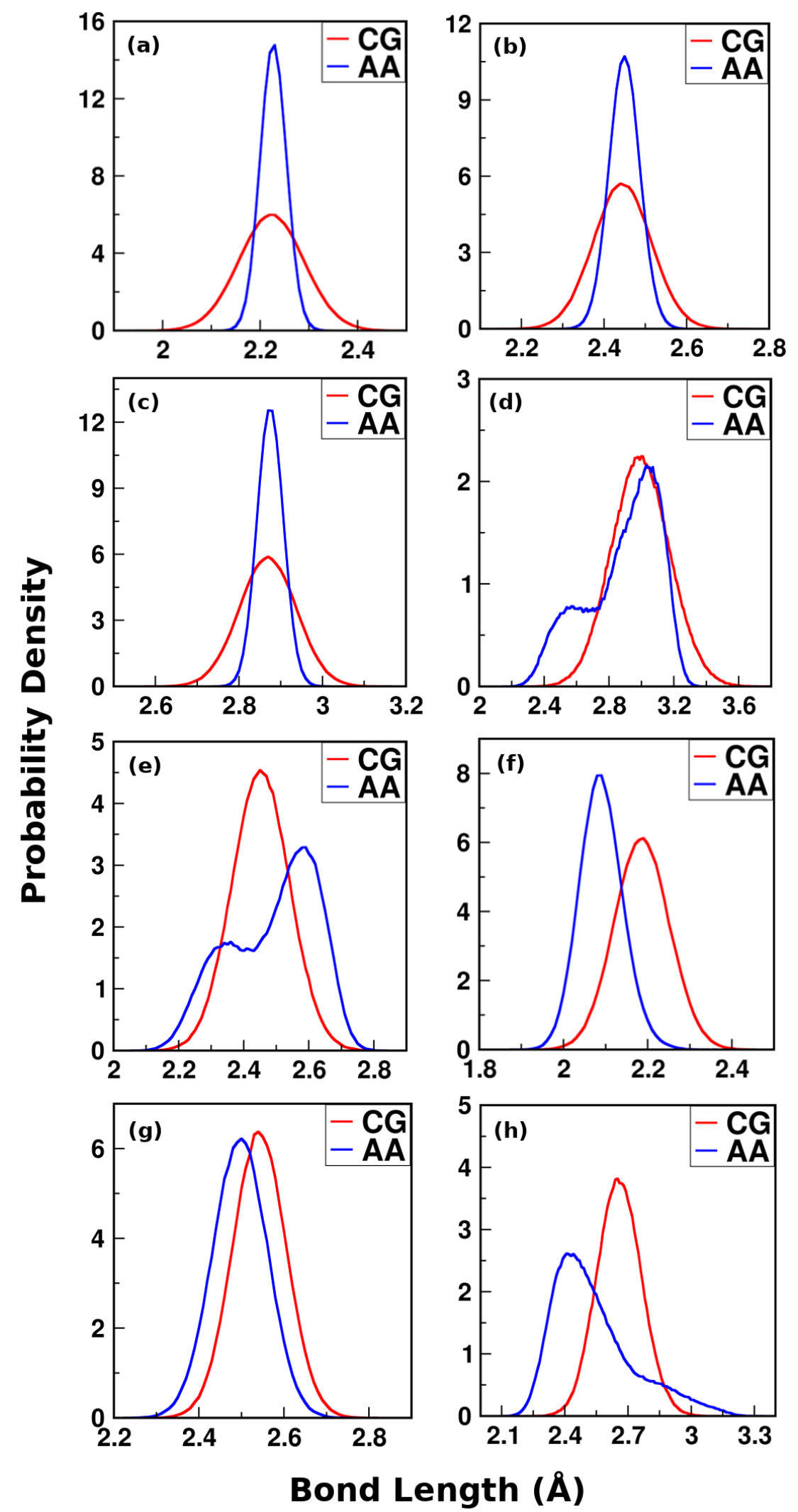

Figure C15.3: Distributions of bonds (a) BZF1-BZF2, (b) BZF1-TL2F, (c) BZF2-BZF1, (d) TL2F-NCT, (e) NCT-CO, (f) CO-NC, (g) NC-COO1, and (h) NC-TL2F 

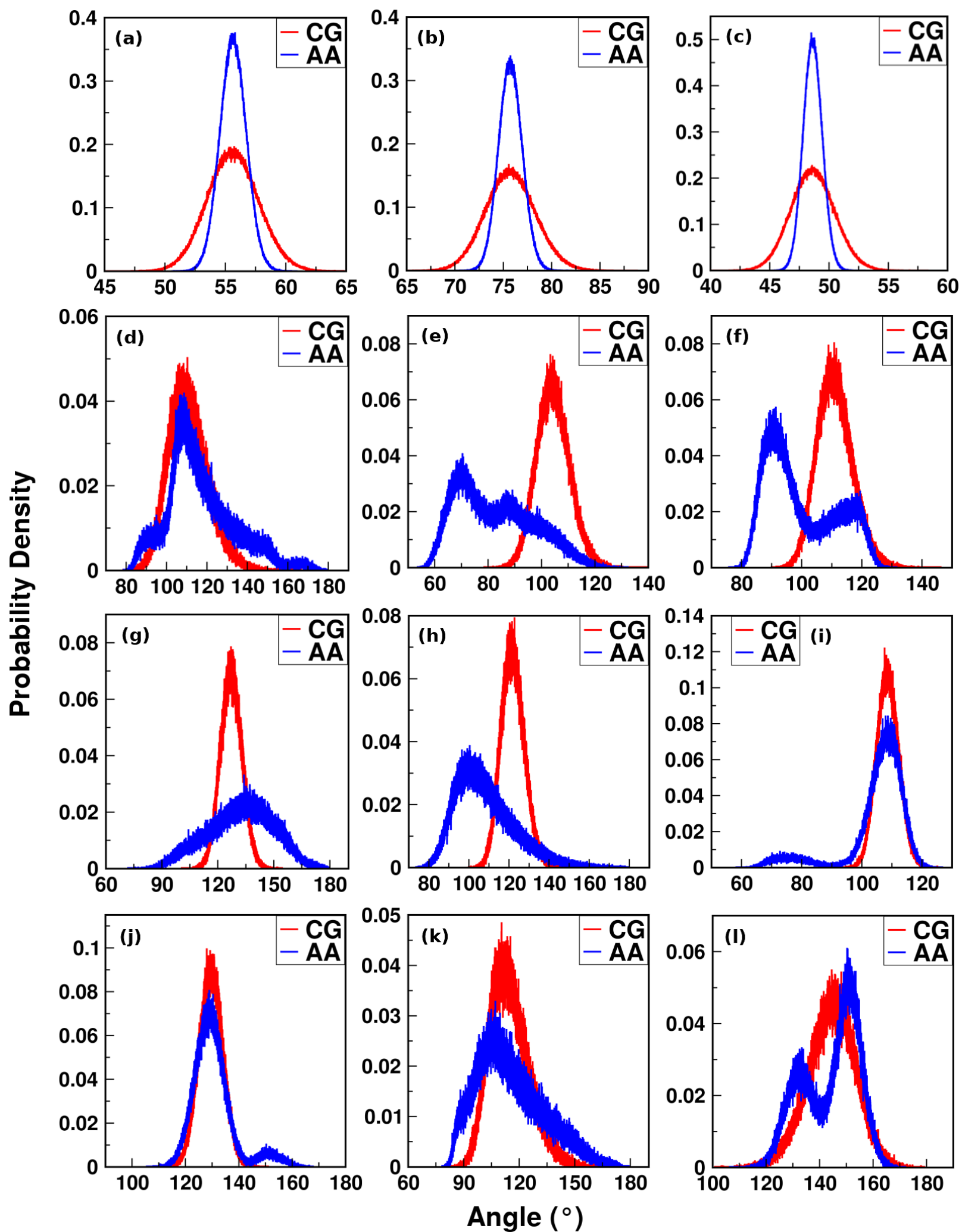

Figure C15.4: Distributions of angles (a) BZF2-BZF1-TL2F, (b) BZF1-BZF2-TL2F (c) BZF1-TL2F-BZF2, (d) BZF2-TL2F-NCT, (e) TL2F-NCT-CO, (f) NCT-CO-NC, (g) CO-NC-TL2F, (h) CO-NC-COO1, (i) COO1NC-TL2F, (j) NC-TL2F-BZF1, (k) NC-TL2F-BZF2, and (I) BZF1-TL2F-NCT 

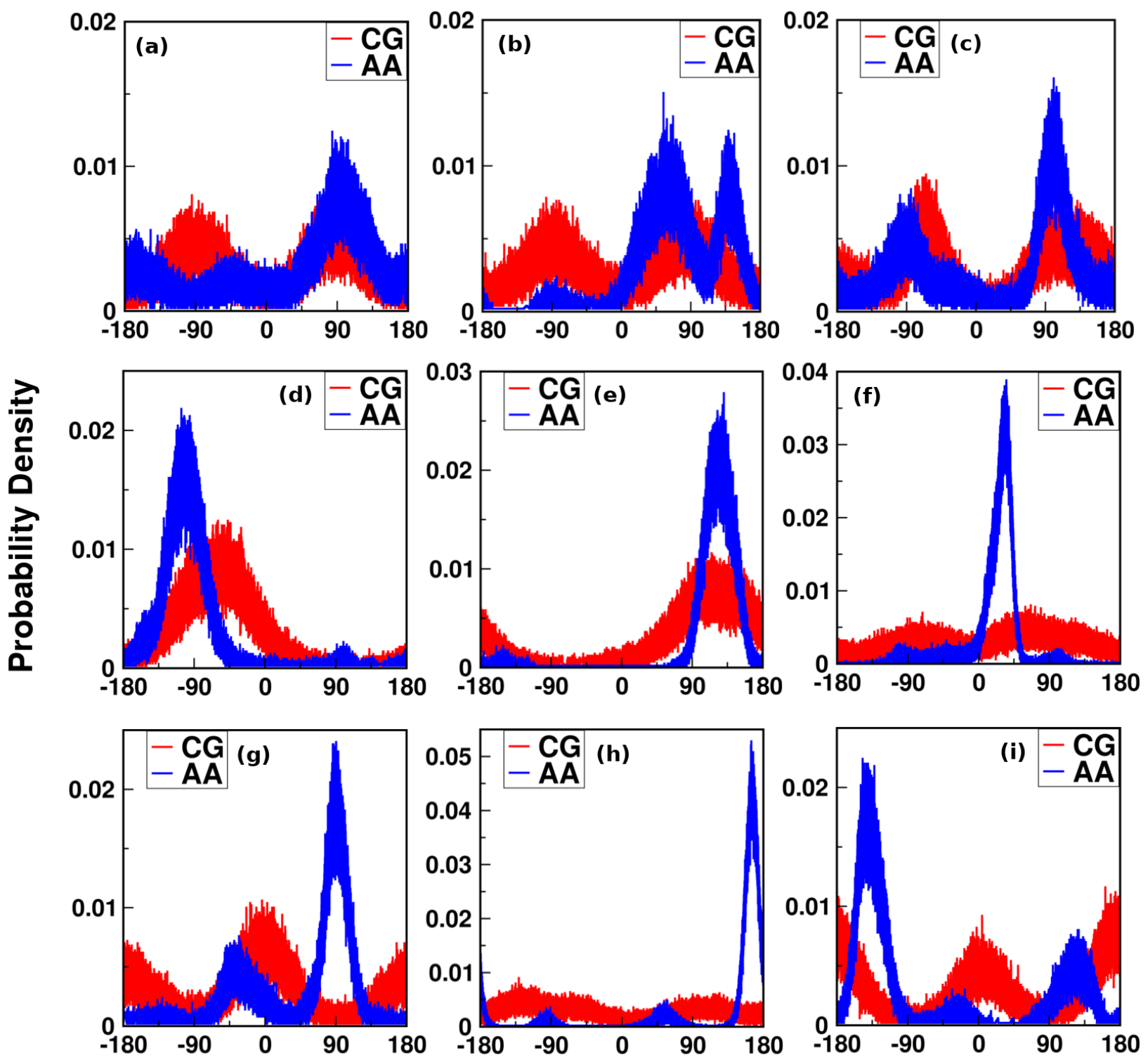

Dihedral $\left({ }^{\circ}\right)$

Figure C15.5: Distributions of dihedrals (a) BZF1-TL2F-NCT-CO, (b) BZF2-TL2F-NCT-CO, (c) TL2FNCT-CO-NC, (d) NCT-CO-NC-TL2F, (e) NCT-CO-NC-COO1, (f) CO-NC-TL2F-BZF1, (g) CO-NC-TL2FBZF2, (h) BZF1-TL2F-NC-COO1, and (i) BZF2-TL2F-NC-COO1 

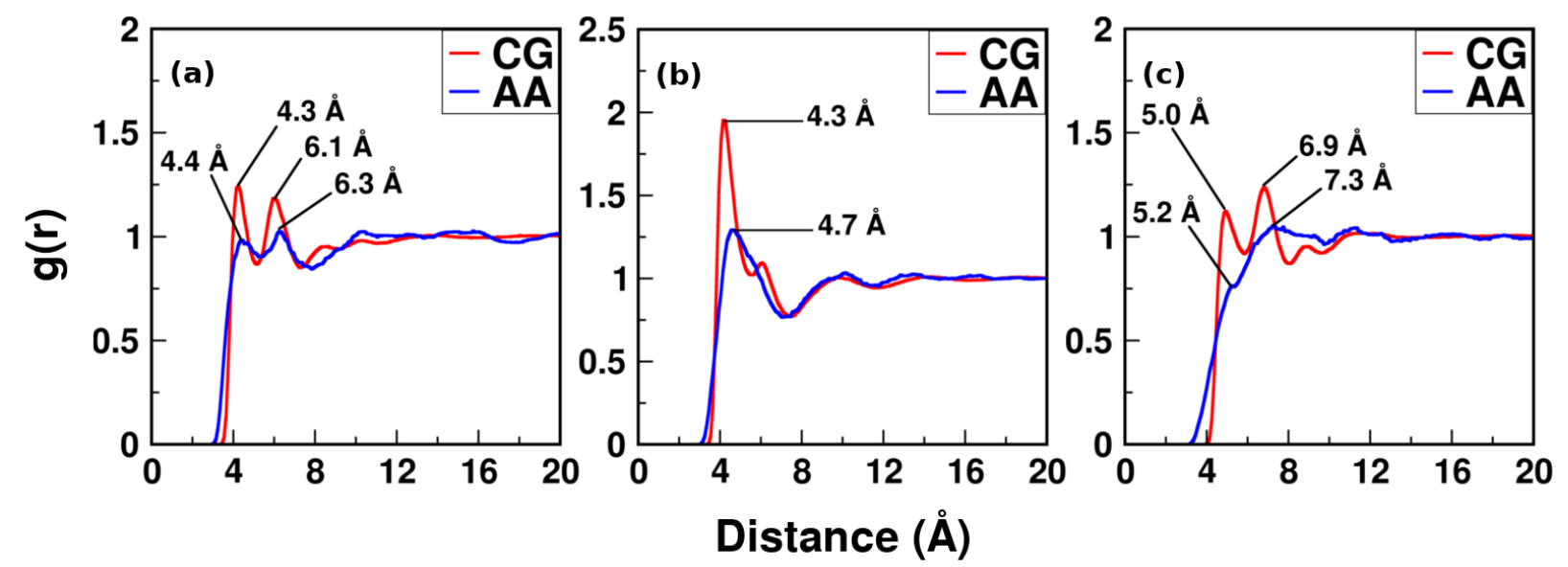

Figure C15.6: RDFs of (a) BZF2, (b) BZF1, and (c) TL2F

\section{Analogue: Toluene}

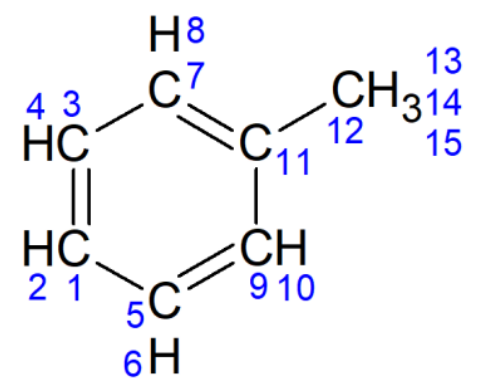

Figure C15.7: All-atom toluene with atom numbering

\section{TL2F}

\section{BZF1}

\section{BZF2}

Figure C15.8: CG model of toluene showing bead types
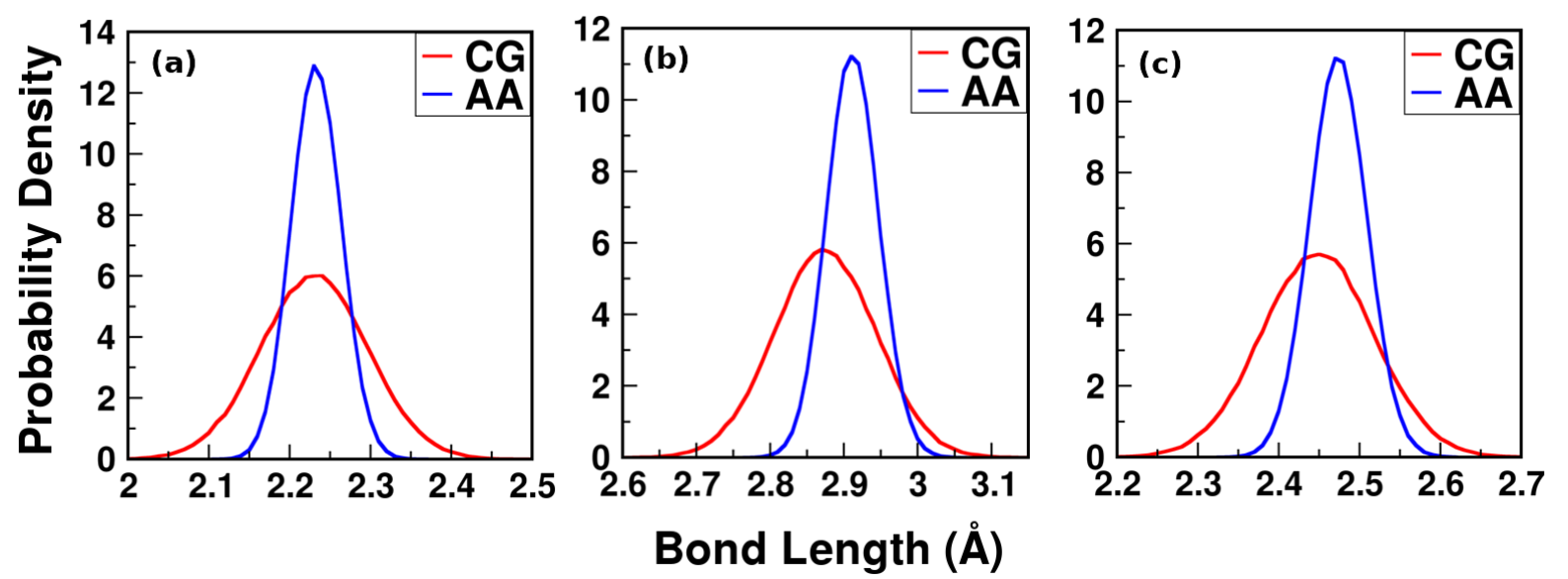

Figure C15.9: Distribution of bonds (a) BZF1-BZF2, (b) BZF1-TL2F, and (c) BZF2-TL2F 

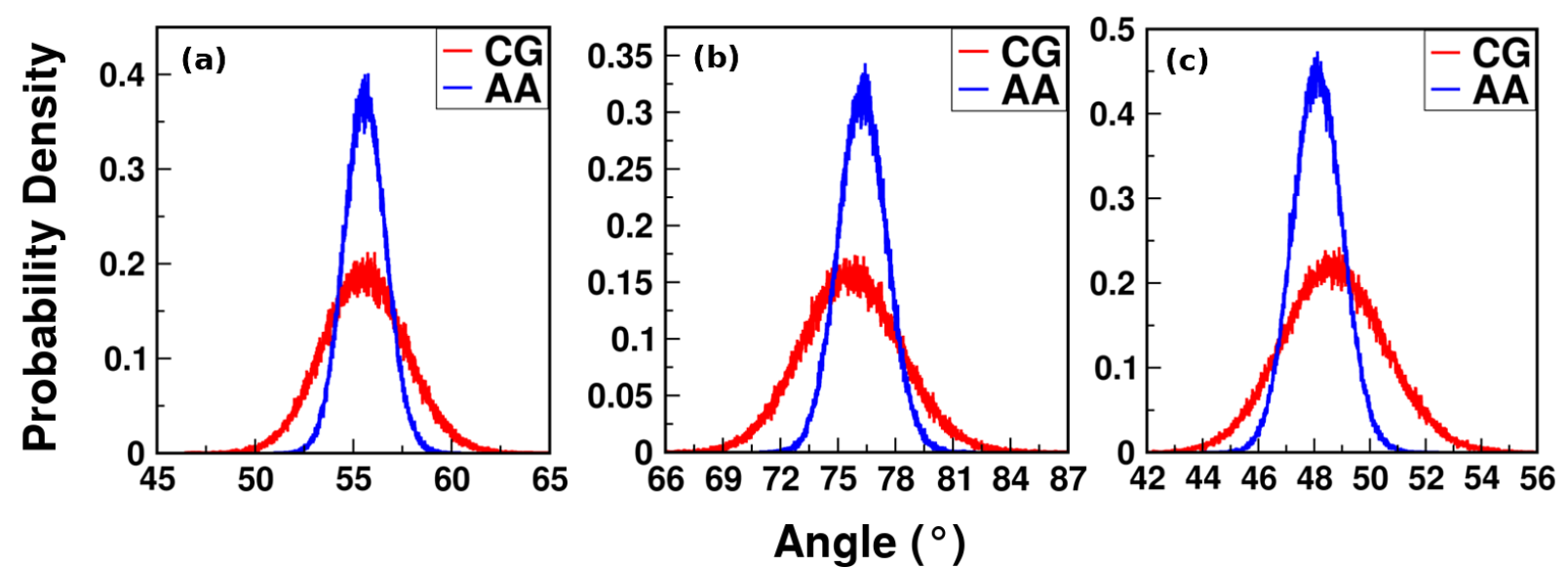

Figure C15.10: Distributions of angles (a) BZF2-BZF1-TL2F, (b) BZF1-BZF2-TL2F, and (c) BZF1-TL2FBZF2
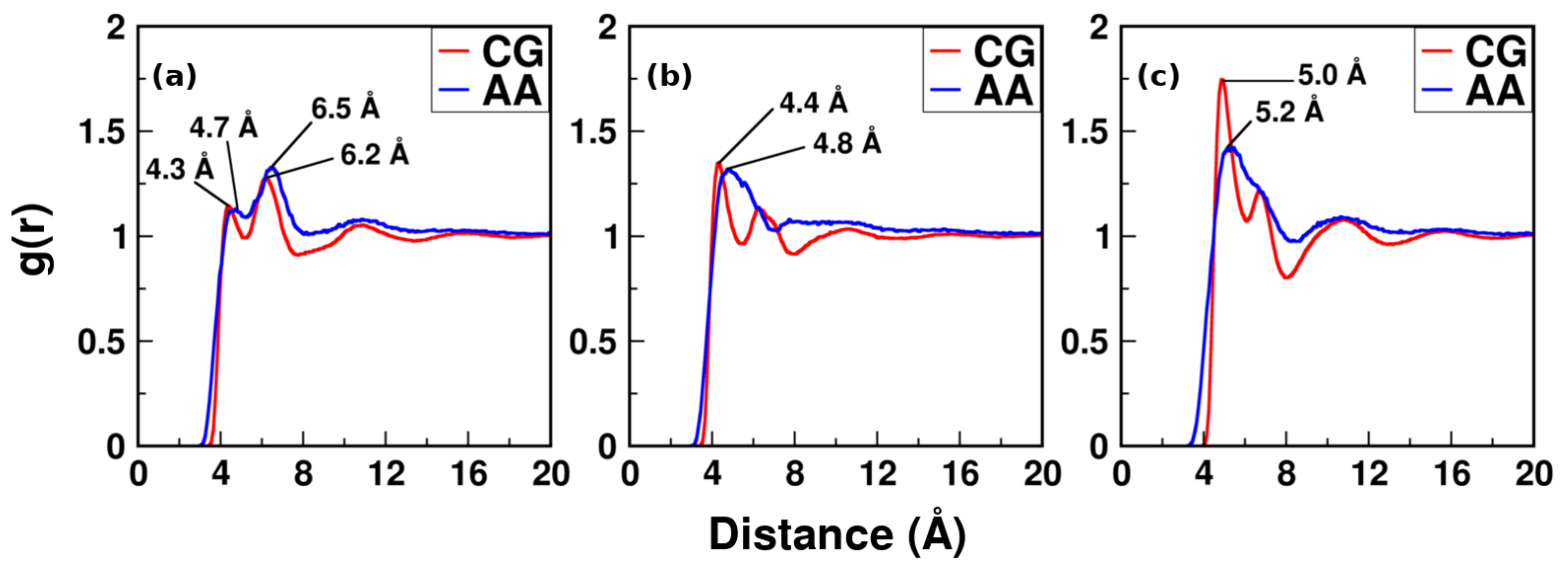

Figure C15.11: RDFs of (a) BZF2, (b) BZF1, and (c) TL2F 


\section{C16: Proline}

\section{Val-Pro-Leu Tripeptide}

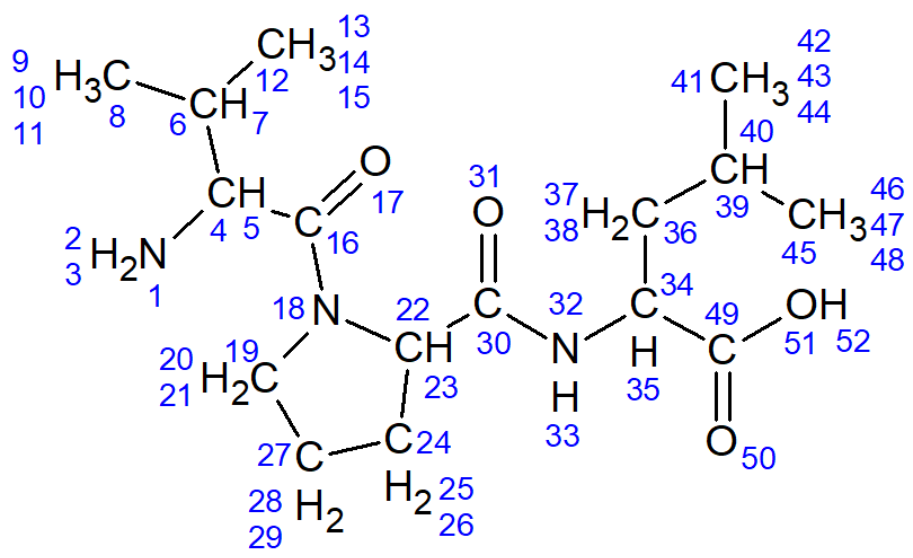

Figure C16.1: All-atom Val-Pro-Leu tripeptide with atom numbering

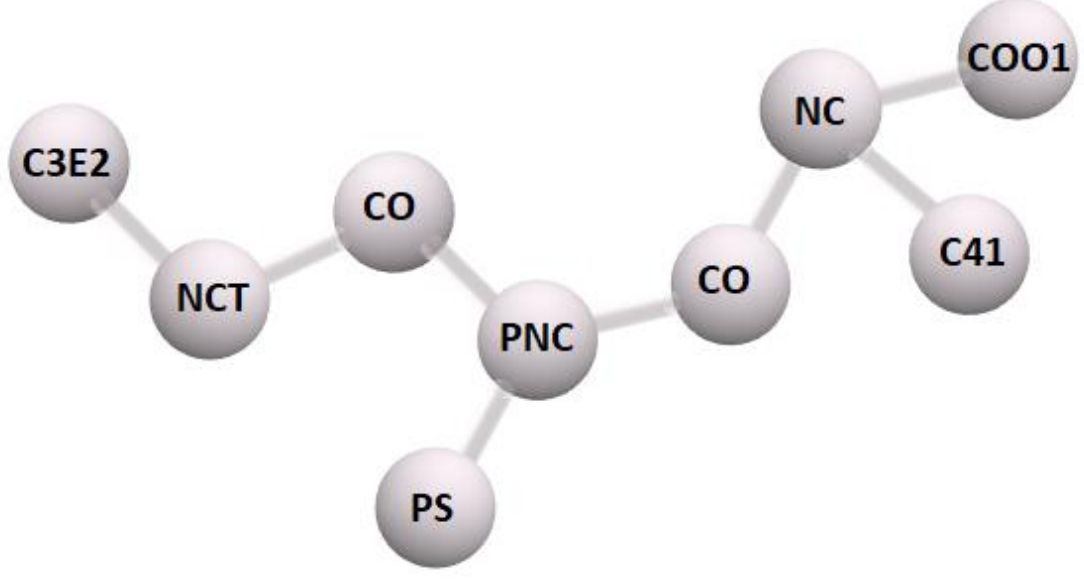

Figure C16.2: CG model of Val-Pro-Leu tripeptide showing bead types 

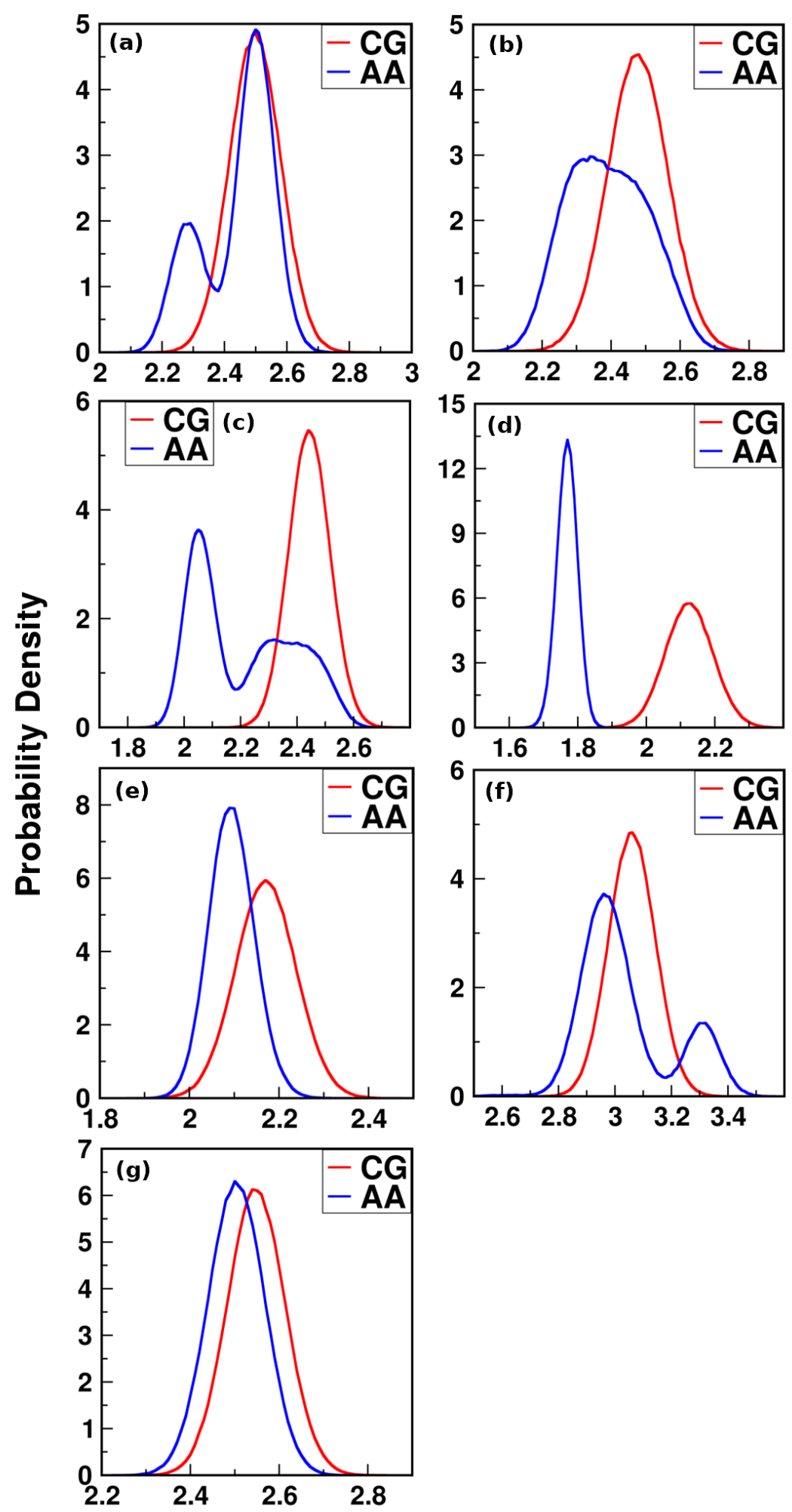

\section{Bond Length $(\AA)$}

Figure C16.3: Distributions of bonds (a) C3E2-NCT, (b) NCT-CO, (c) CO-PNC, (d) PNC-PS, (e) CO-NC, (f) NC-C41, and (g) NC-COO1 

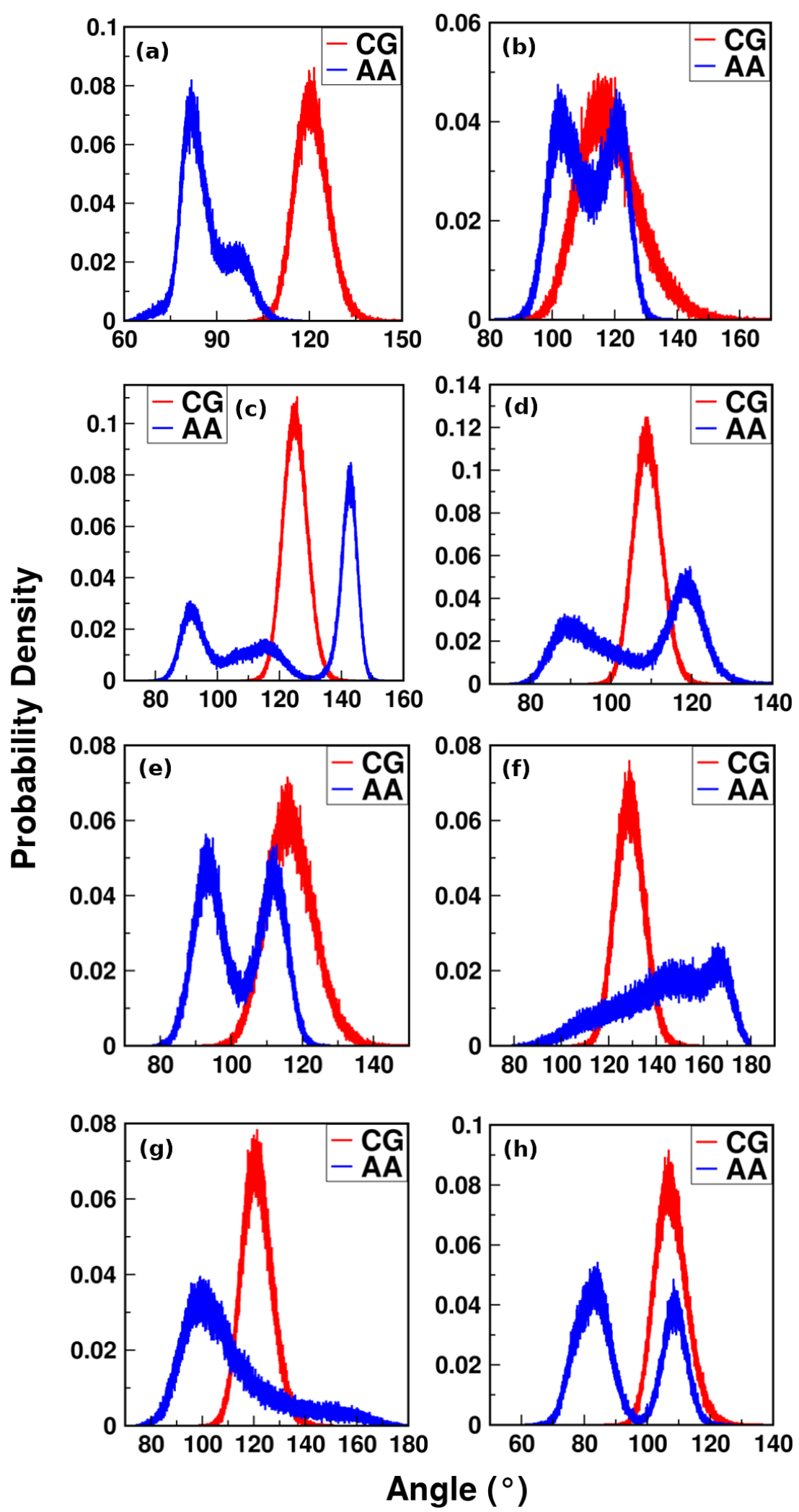

Figure C16.4: Distributions of angles (a) C3E2-NCT-CO, (b) NCT-CO-PNC, (c) CO-PNC-PS, (d) COPNC-CO, (e) PNC-CO-NC, (f) CO-NC-C41, (g) CO-NC-COO1, and (h) C41-NC-COO1 

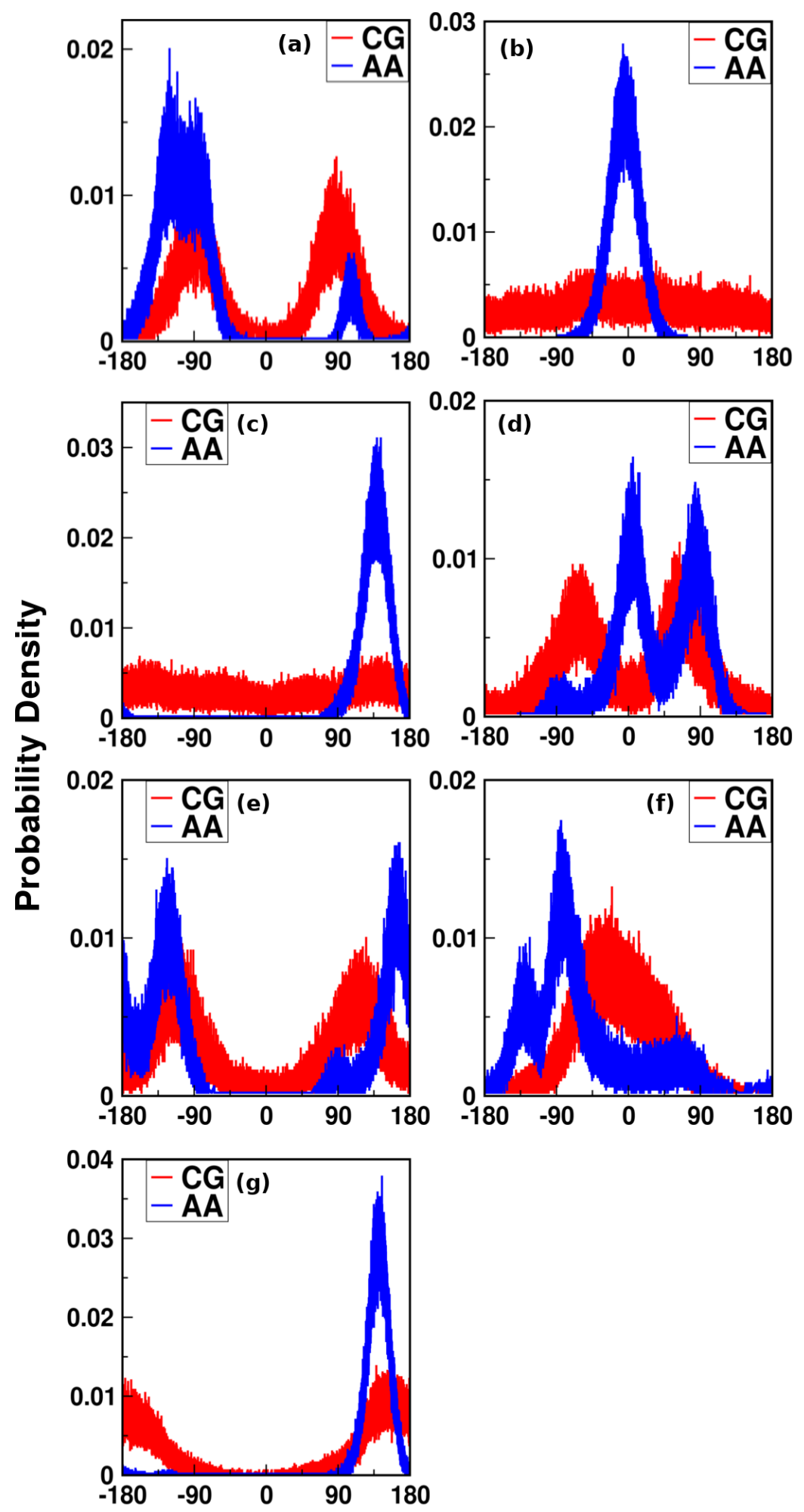

Dihedral $\left({ }^{\circ}\right)$

Figure C16.5: Distributions of dihedrals (a) C3E2-NCT-CO-PNC, (b) NCT-CO-PNC-PS (c) NCT-COPNC-CO, (d) CO-PNC-CO-NC, (e) PS-PNC-CO-NC, (f) PNC-CO-NC-C41, and (g) PNC-CO-NC-COO1 


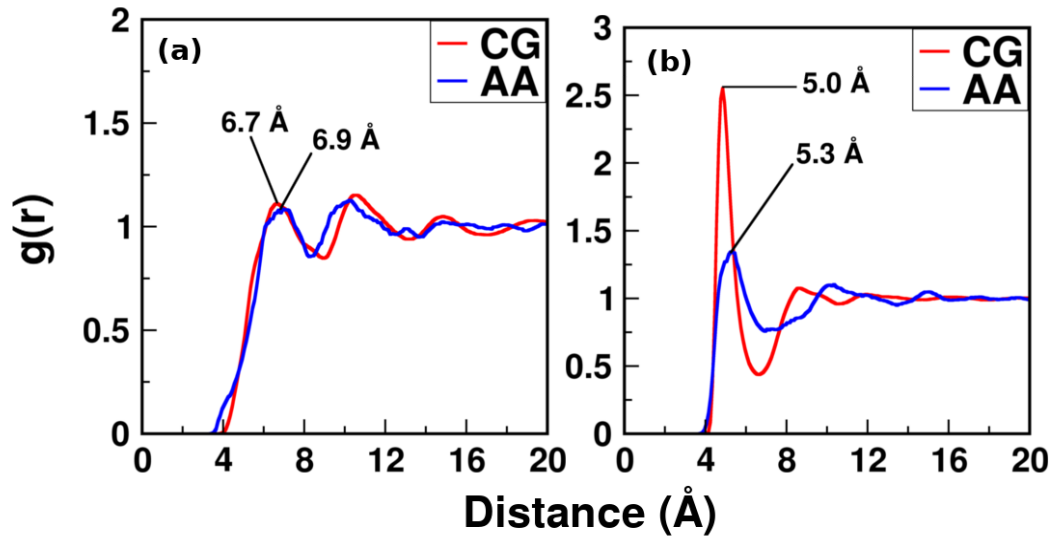

Figure C16.6: RDFs of (a) PNC and (b) PS 


\section{C17: Serine}

\section{Ser-Ser Dipeptide}

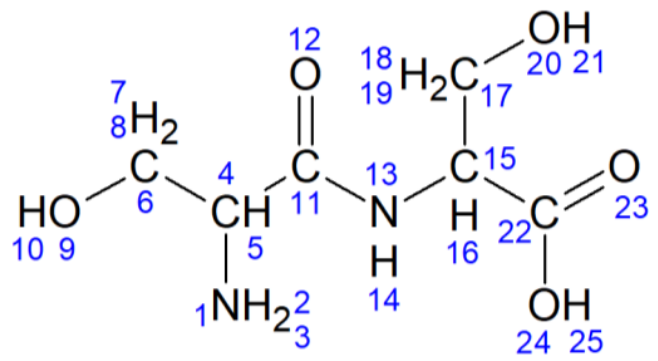

Figure C17.1: All-atom Serine dipeptide with atom numbering

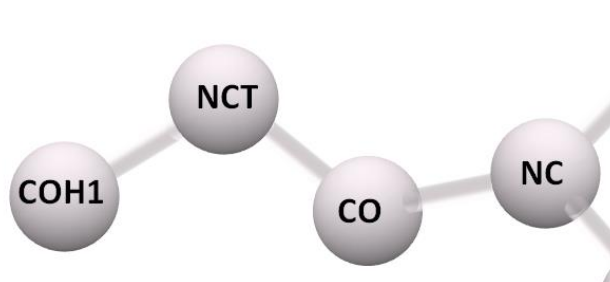

$\mathrm{COH} 1$

\section{CT}

Figure C17.2: CG model of Serine dipeptide showing bead types
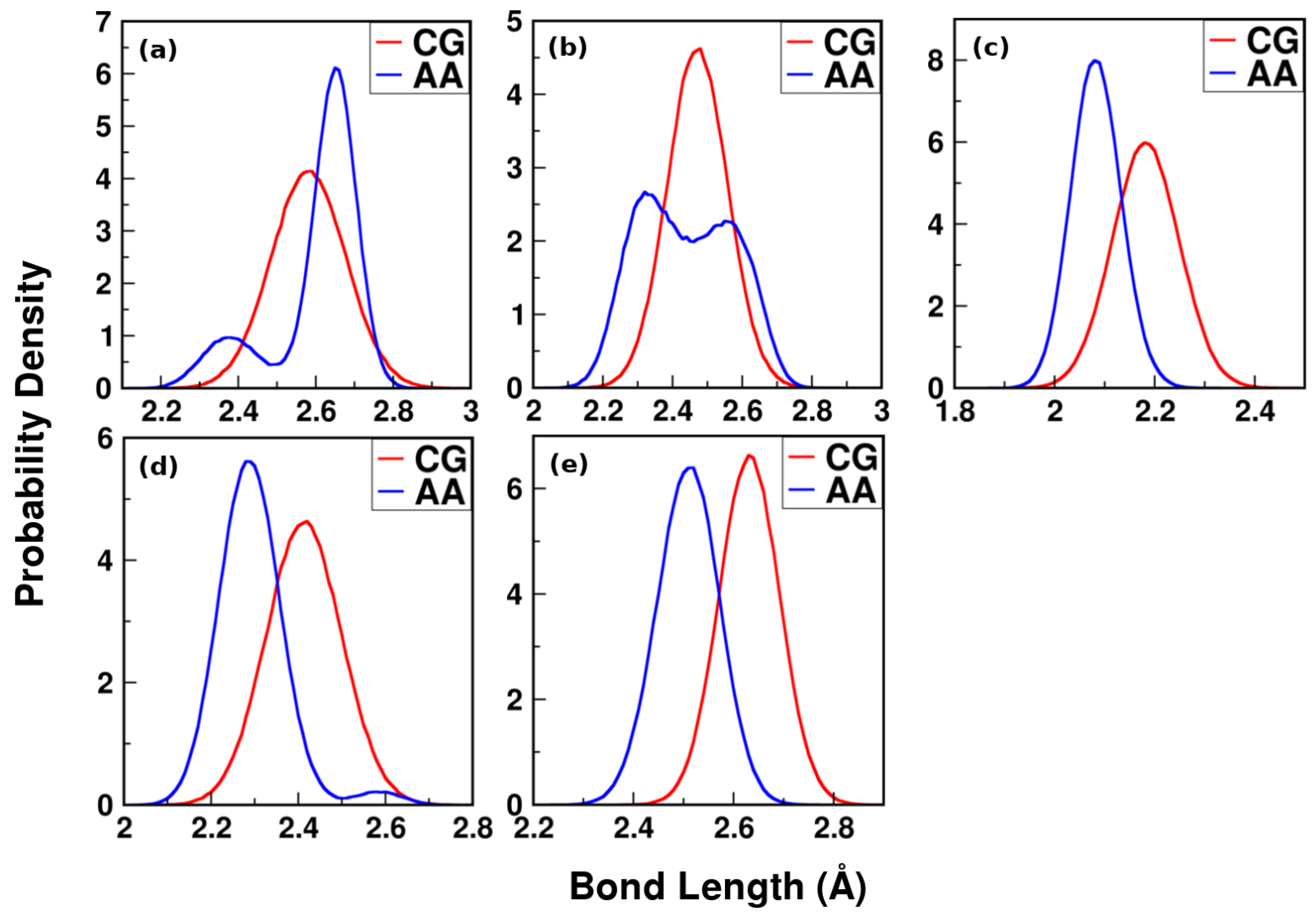

Figure C17.3: Distributions of bonds (a) COH1-NCT, (b) NCT-CO, (c) CO-NC, (d) NC-COH1, and (e) NCCOO1 

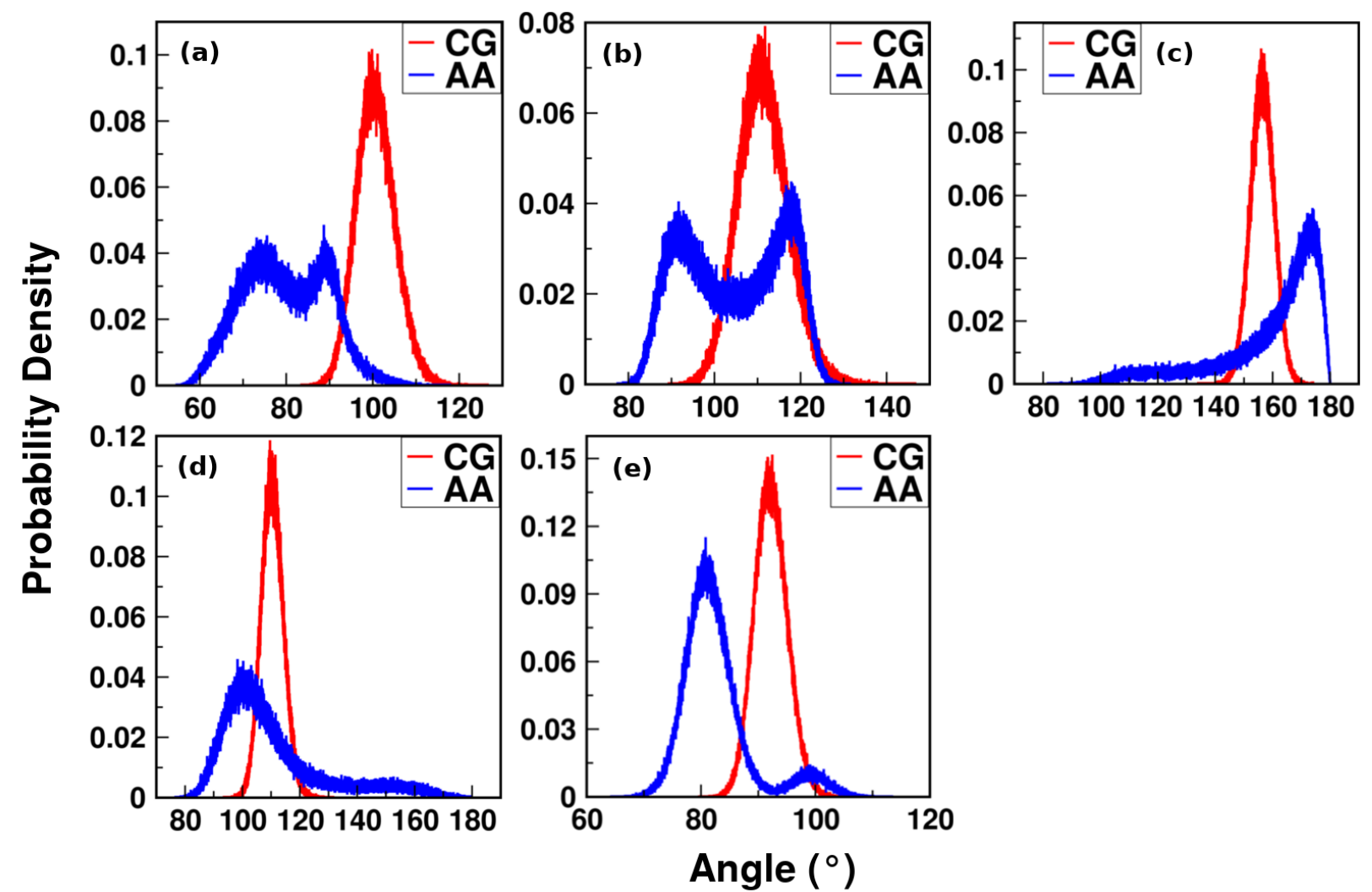

Figure C17.4: Distributions of angles (a) COH1-NCT-CO, (b) NCT-CO-NC, (c) CO-NC-COH1, (d) CO$\mathrm{NC}-\mathrm{COO} 1$, and (e) $\mathrm{COH} 1-\mathrm{NC}-\mathrm{COO} 1$
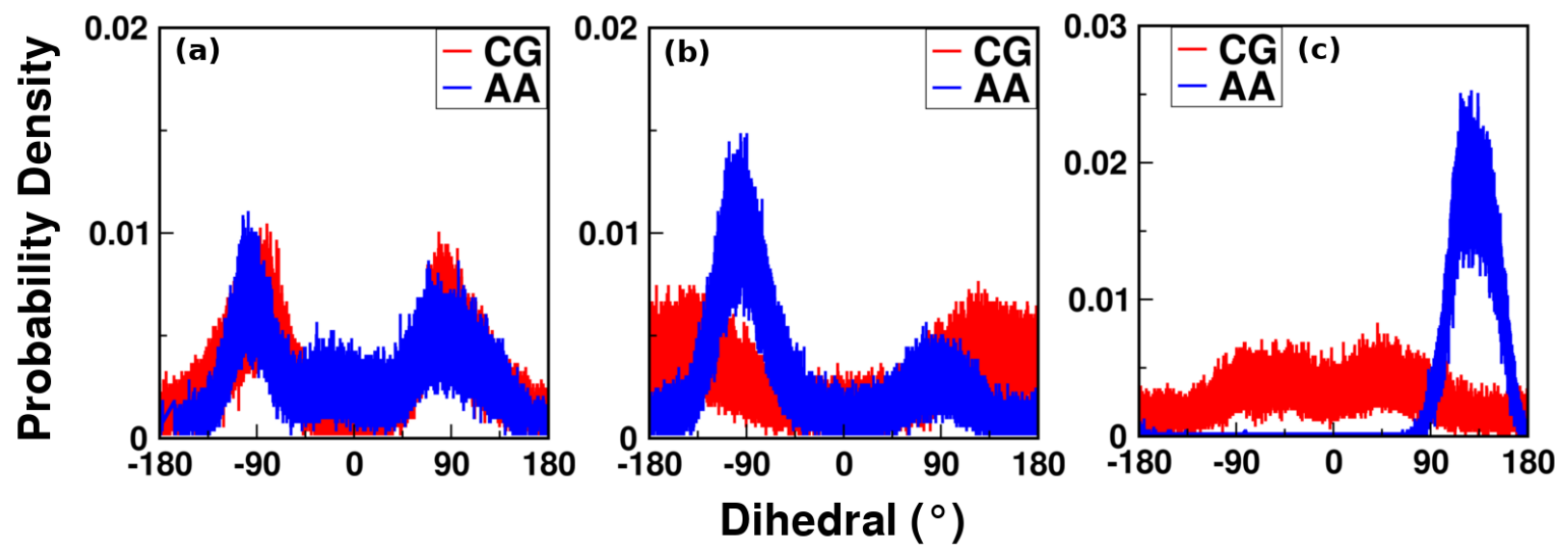

Figure C17.5: Distributions of dihedrals (a) COH1-NCT-CO-NC, (b) NCT-CO-NC-COH1, and (c) NCTCO-NC-COO1 


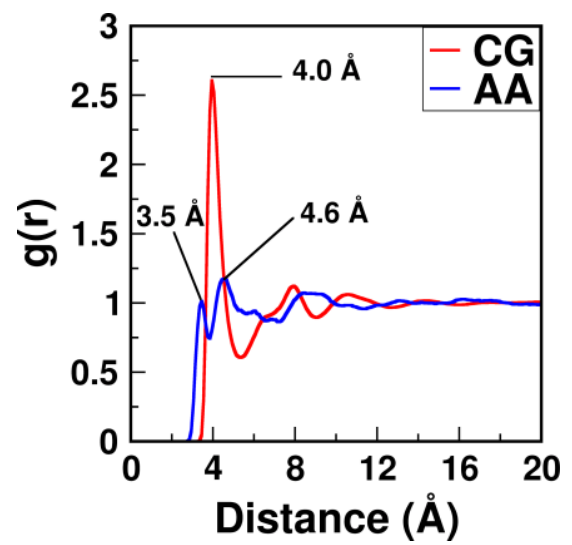

Figure C17.6: $\mathrm{RDF}$ of $\mathrm{COH} 1$

\section{Analogue: Methanol}<smiles>CO</smiles>

Figure C17.7: All-atom methanol

\section{$\mathrm{COH} 1$}

Figure C17.8: CG model of methanol showing bead type

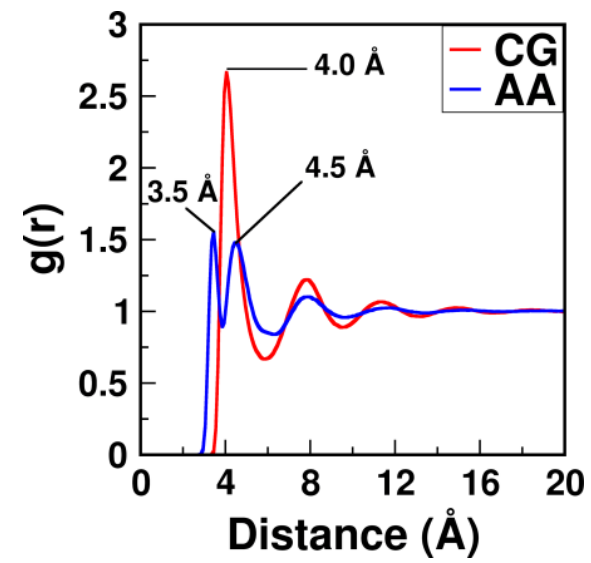

Figure C17.9: $\mathrm{RDF}$ of $\mathrm{COH} 1$ 


\section{C18: Threonine}

\section{Thr-Thr Dipeptide}

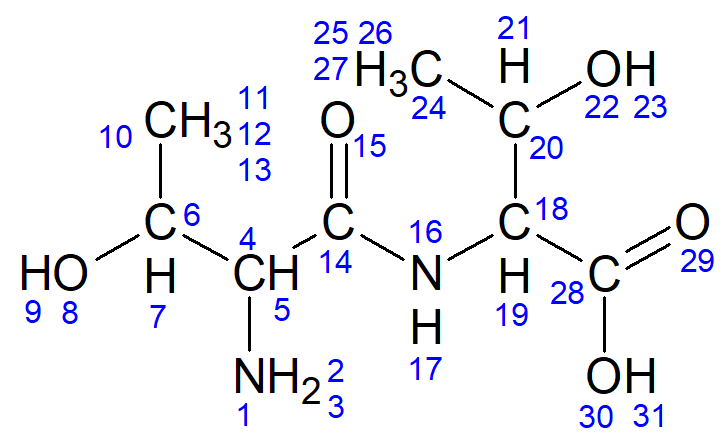

Figure C18.1: All-atom model of Threonine dipeptide with atom numbering

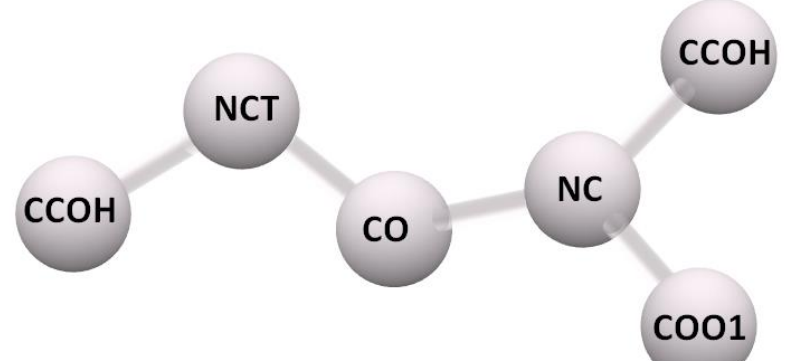

Figure C18.2: CG model of Threonine dipeptide
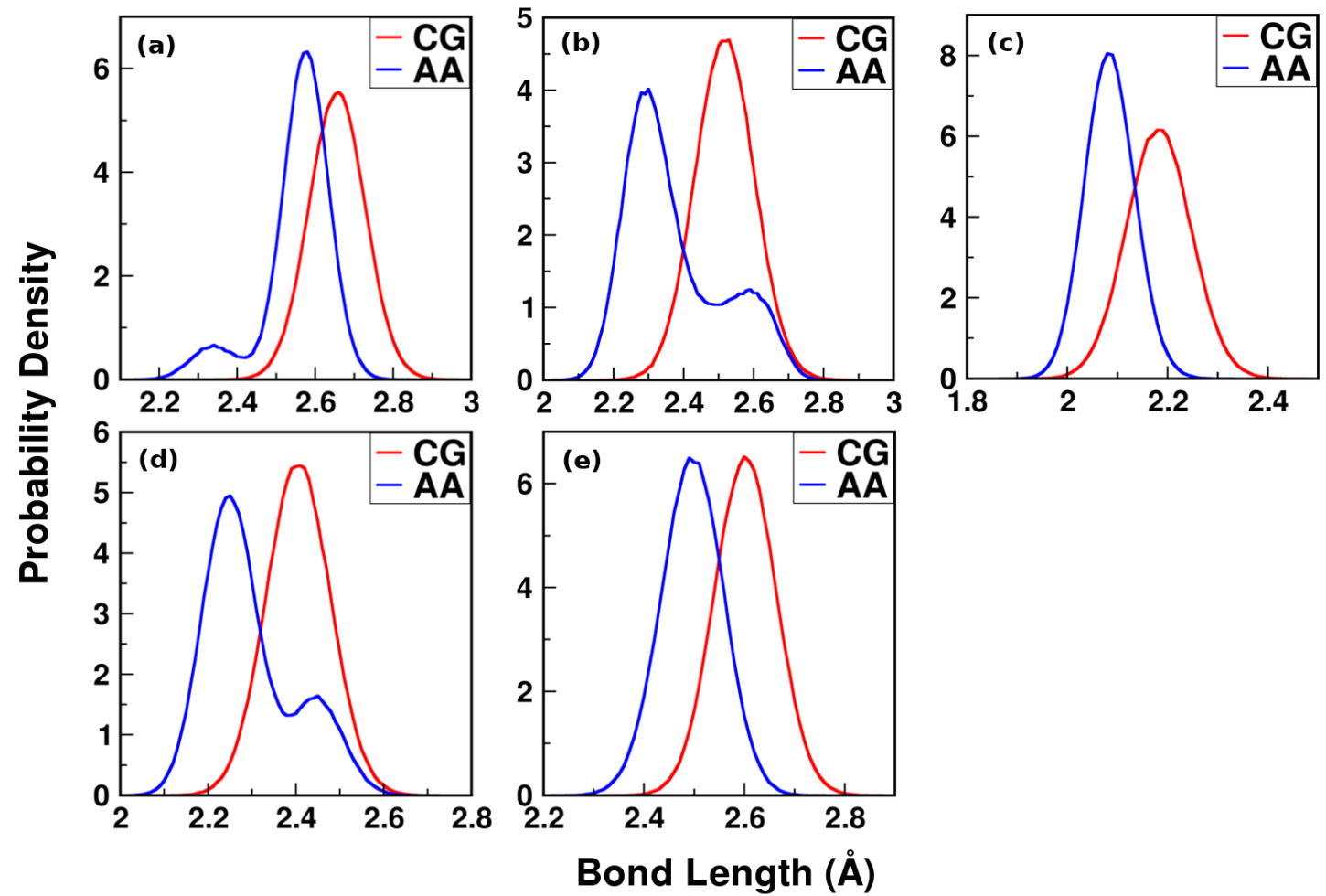

Figure C18.3: Distributions of bonds (a) CCOH-NCT, (b) NCT-CO, (c) CO-NC, (d) NC-CCOH, and (e) NC-COO1 

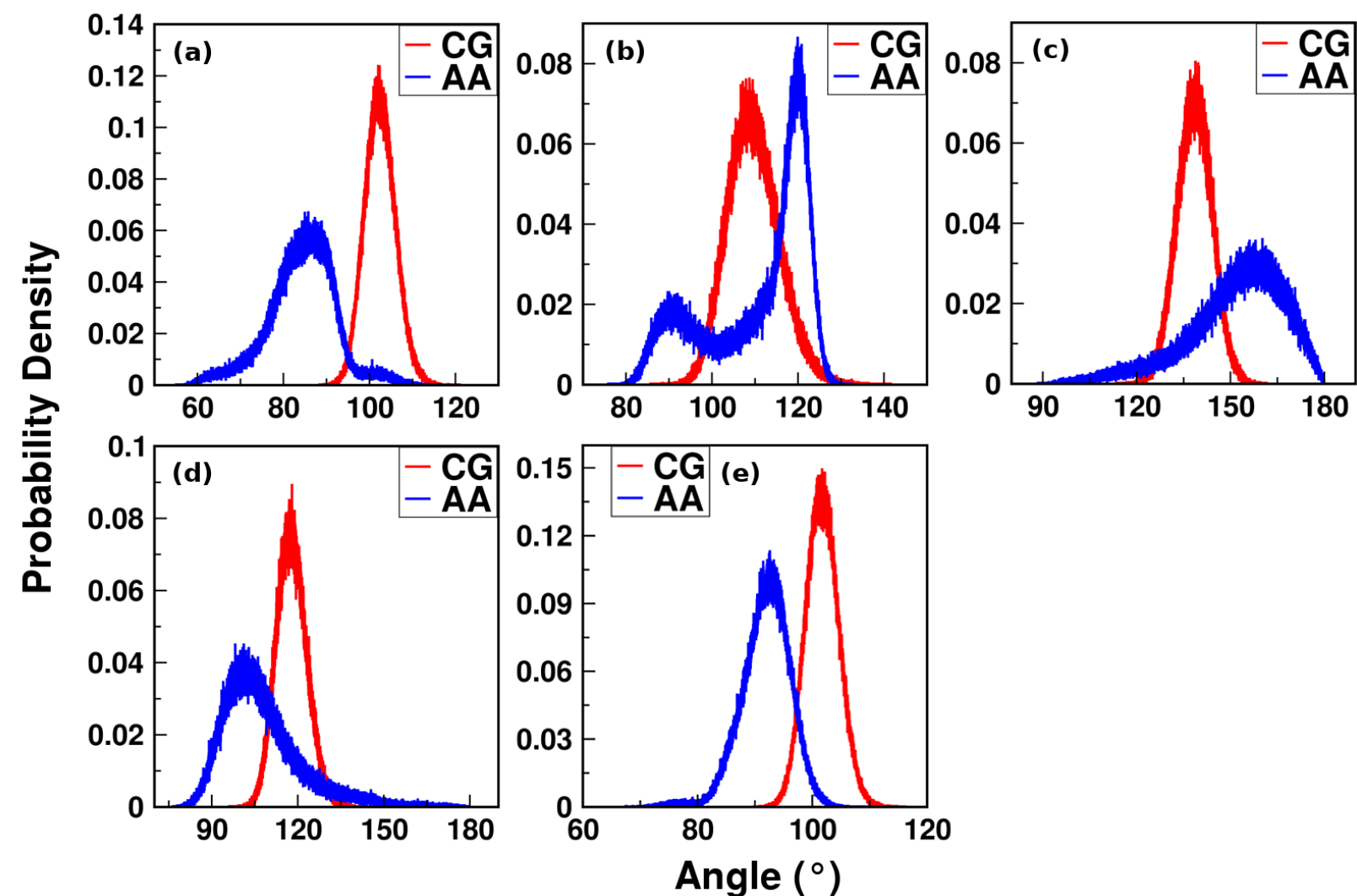

Figure C18.4: Distributions of angles (a) CCOH-NCT-CO, (b) NCT-CO-NC, (c) CO-NC-CCOH, (d) CO$\mathrm{NC}-\mathrm{COO} 1$, and (e) $\mathrm{CCOH}-\mathrm{NC}-\mathrm{COO} 1$
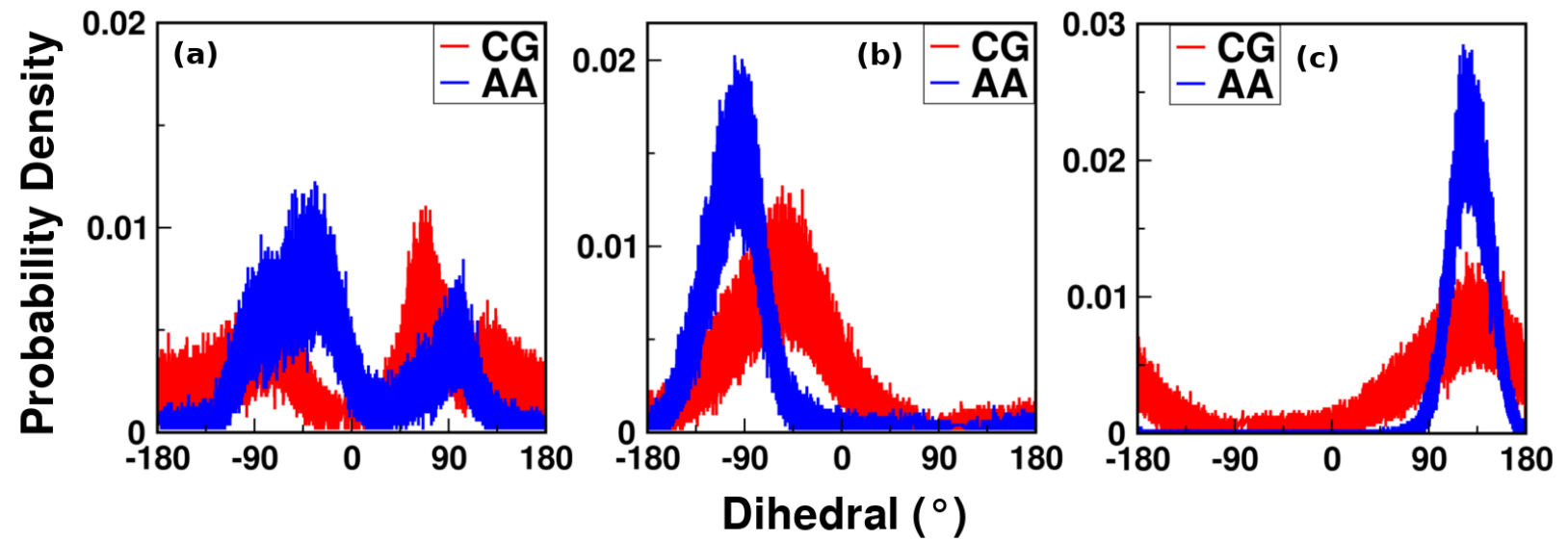

Figure C18.5: Distribution of dihedrals (a) CCOH-NCT-CO-NC, (b) NCT-CO-NC-CCOH, and (c) NCTCO-NC-COO1 


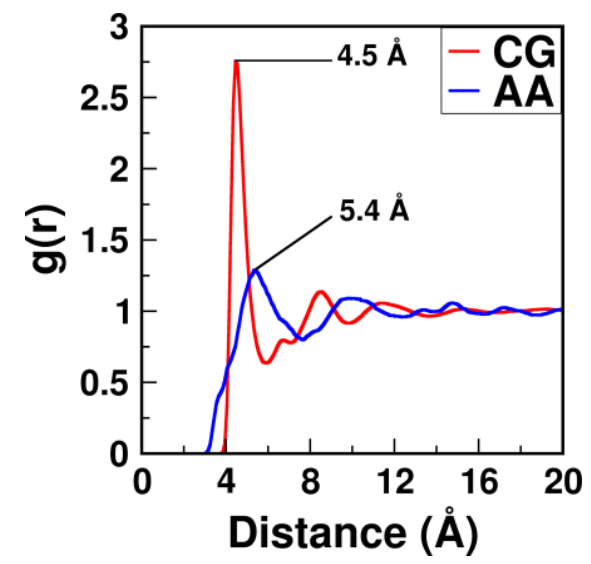

Figure C18.6: $\mathrm{RDF}$ of $\mathrm{CCOH}$

Analogue: Ethanol

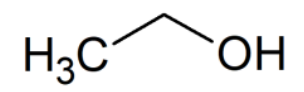

Figure C18.7: All-atom ethanol

\section{$\mathrm{CCOH}$}

Figure C18.8: CG model of ethanol showing bead type

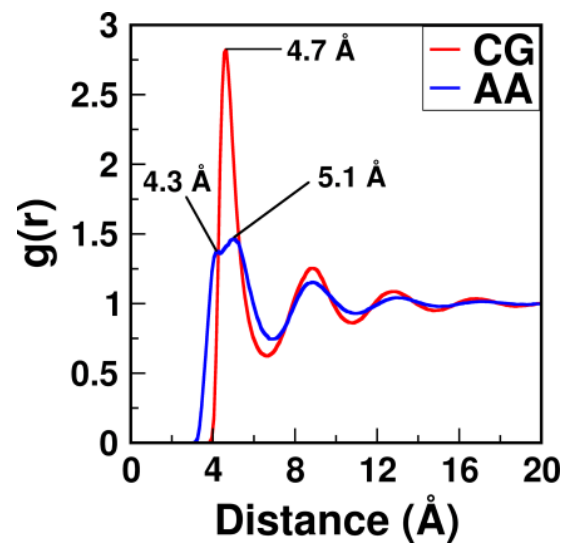

Figure C18.9: RDF of $\mathrm{CCOH}$ 


\section{C19: Tryptophan}

\section{Trp-Trp Dipeptide}

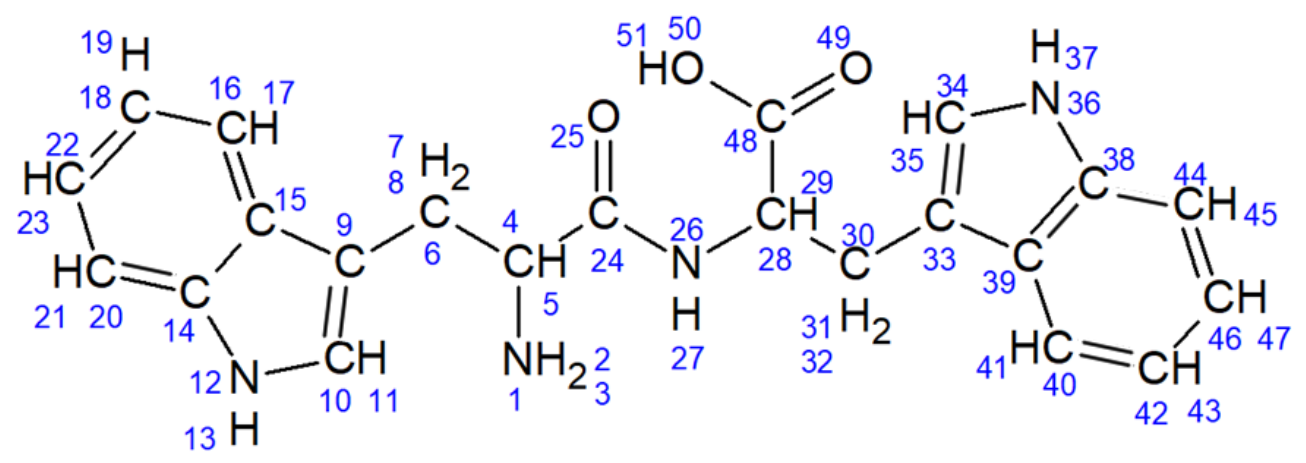

Figure C19.1: All-atom Tryptophan dipeptide with atom numbering

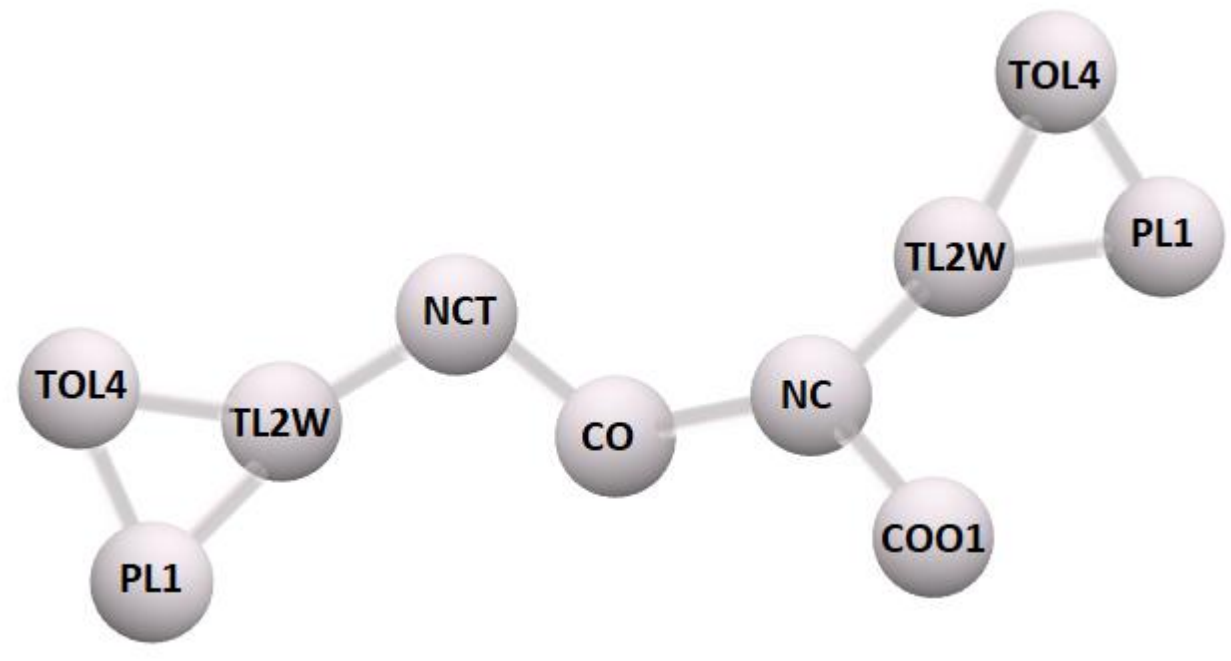

Figure C19.2: CG model of Tryptophan dipeptide showing bead types 

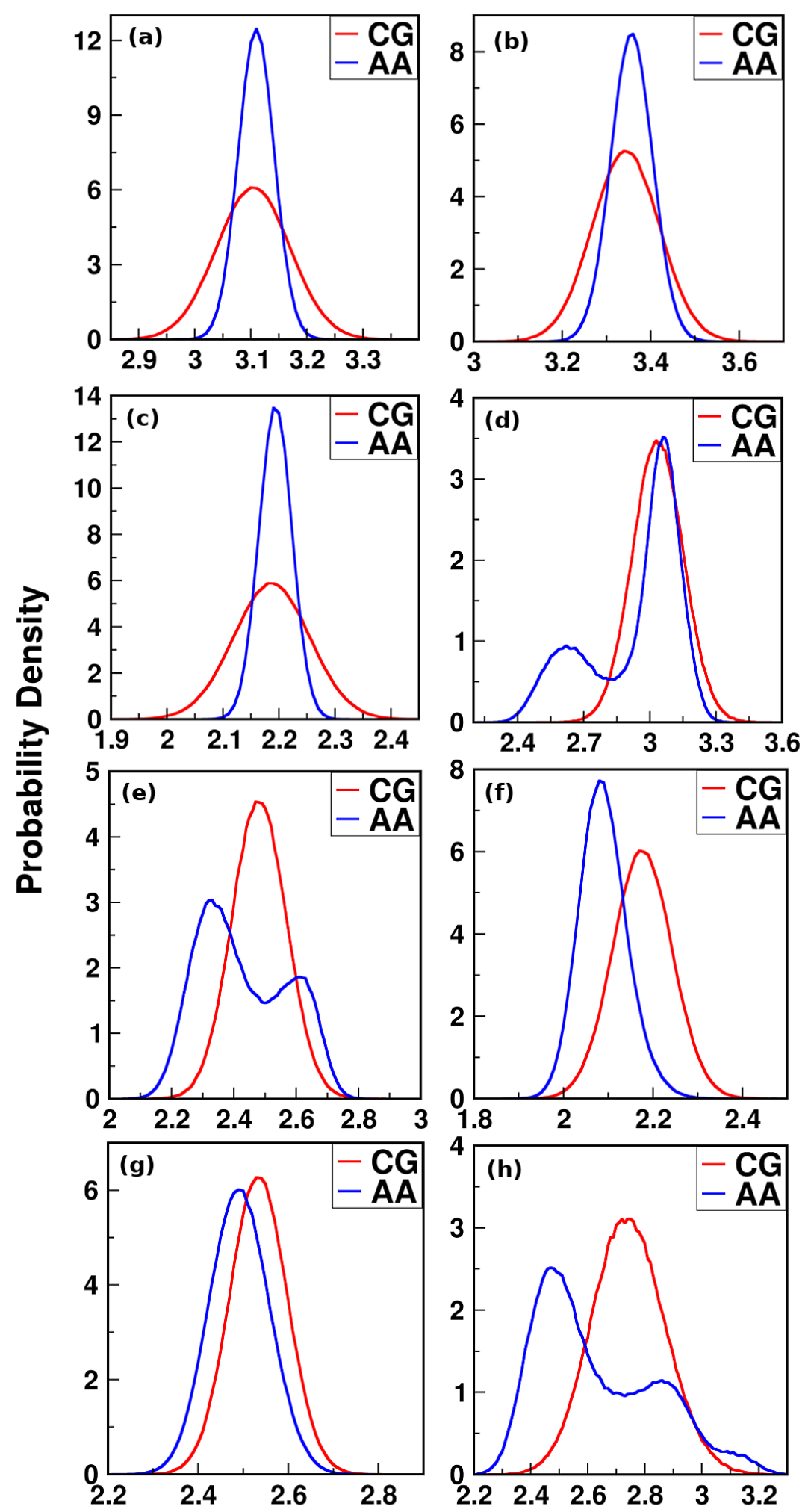

Bond Length ( $\AA$ )

Figure C19.3: Distributions of bonds (a) TOL4-PL1, (b) TOL4-TL2W, (c) TL2W-PL1, (d) TL2W-NCT, (e) NCT-CO, (f) CO-NC, (g) NC-COO1, and (h) NC-TL2W 

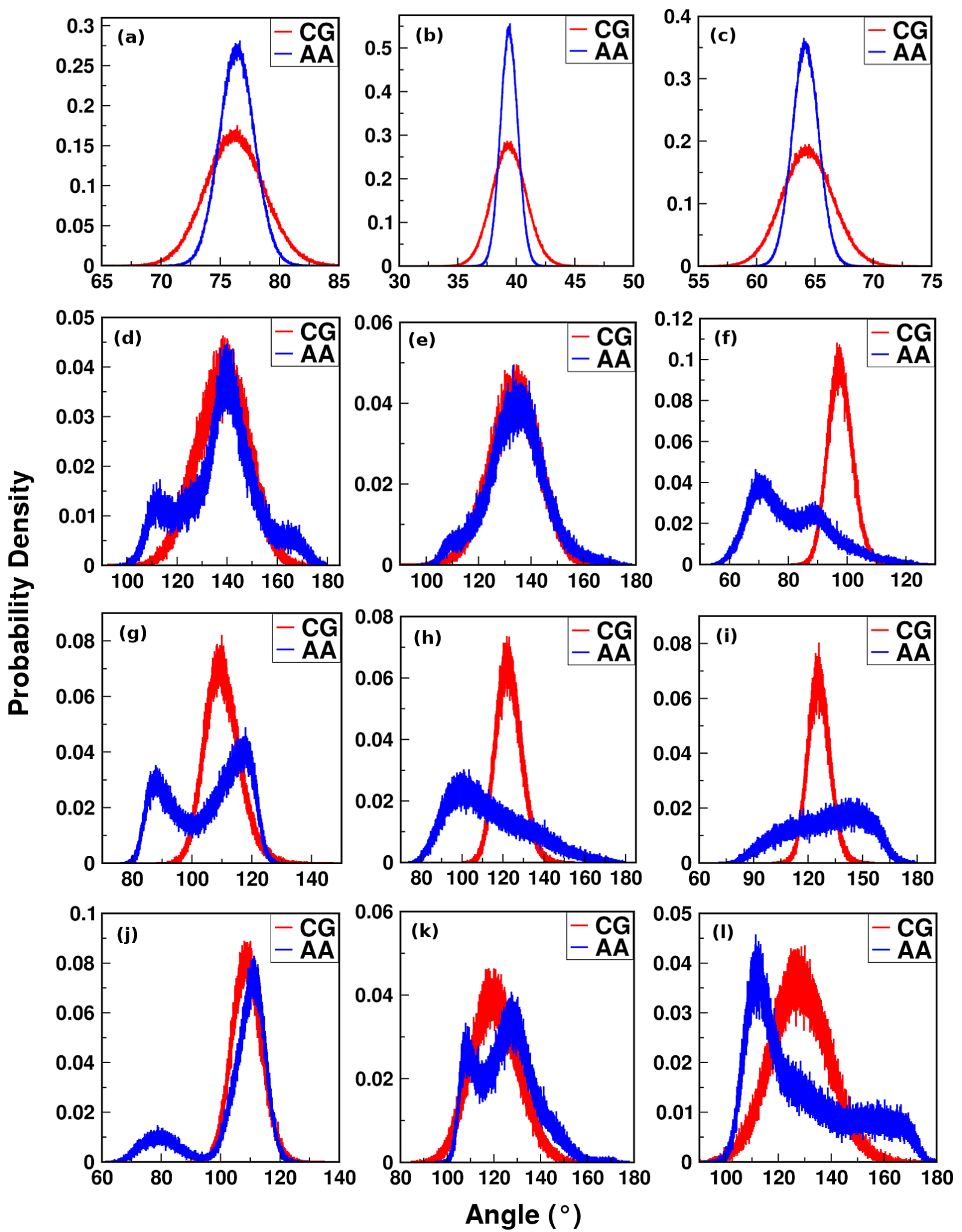

Figure C19.4: Distributions of angles (a) TL2W-PL1-TOL4, (b) PL1-TOL4-TL2W, (c) TOL4-TL2W-PL1, (d) TOL4-TL2W-NCT, (e) PL1-TL2W-NCT, (f) TL2W-NCT-CO, (g) NCT-CO-NC, (h) CO-NC-COO1, (i) CO-NC-TL2W, (j) COO1-NC-TL2W, (k) NC-TL2W-PL1, and (l) NC-TL2W-TOL4 

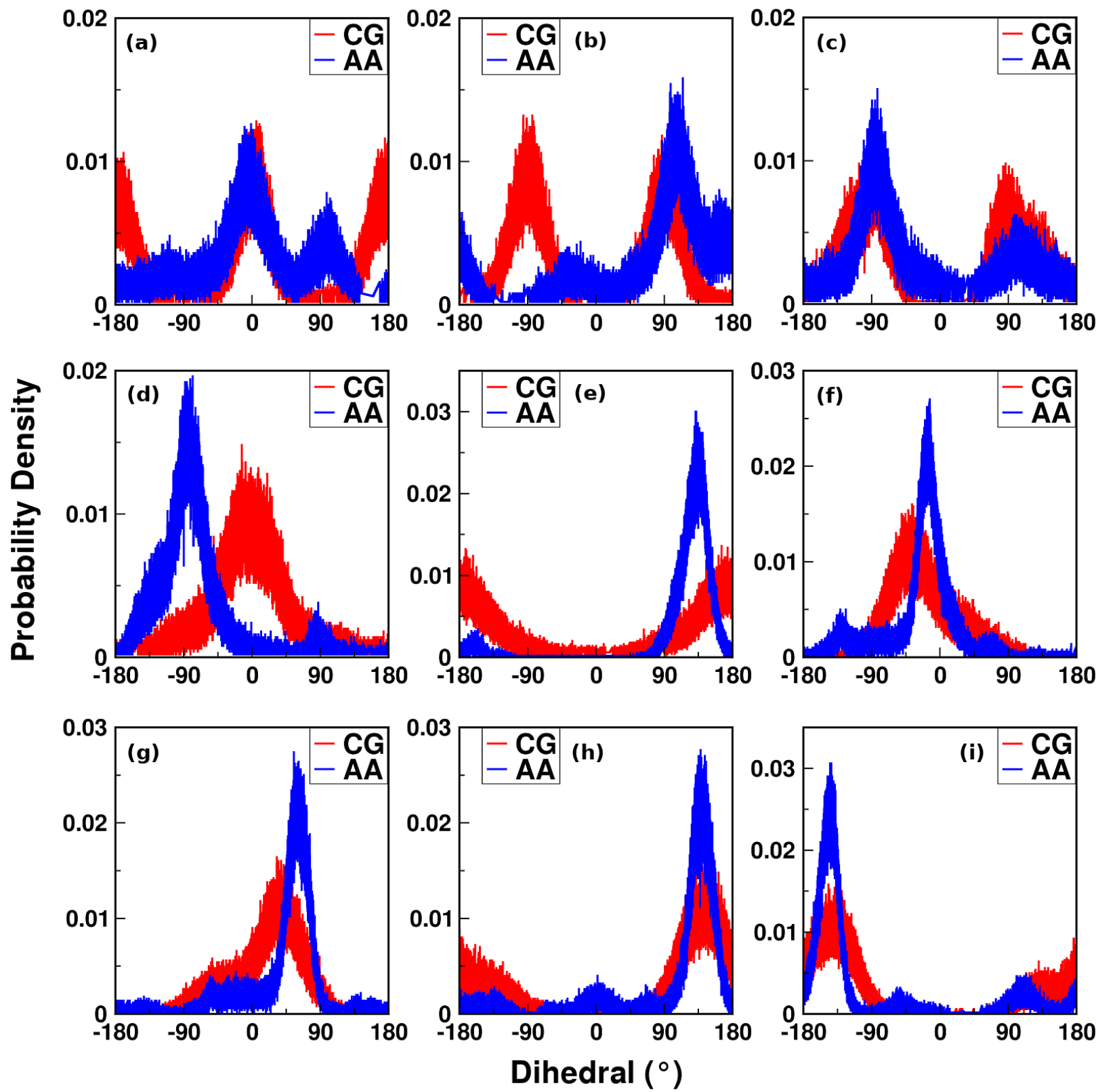

Figure C19.5: Distributions of dihedrals (a) TOL4-TL2W-NCT-CO, (b) PL1-TL2W-NCT-CO, (c) TL2WNCT-CO-NC, (d) NCT-CO-NC-TL2W, (e) NCT-CO-NC-COO1, (f) CO-NC-TL2W-PL1, (g) CO-NC-TL2WTOL4, (h) COO1-NC-TL2W-PL1, and (i) COO1-NC-TL2W-TOL4 

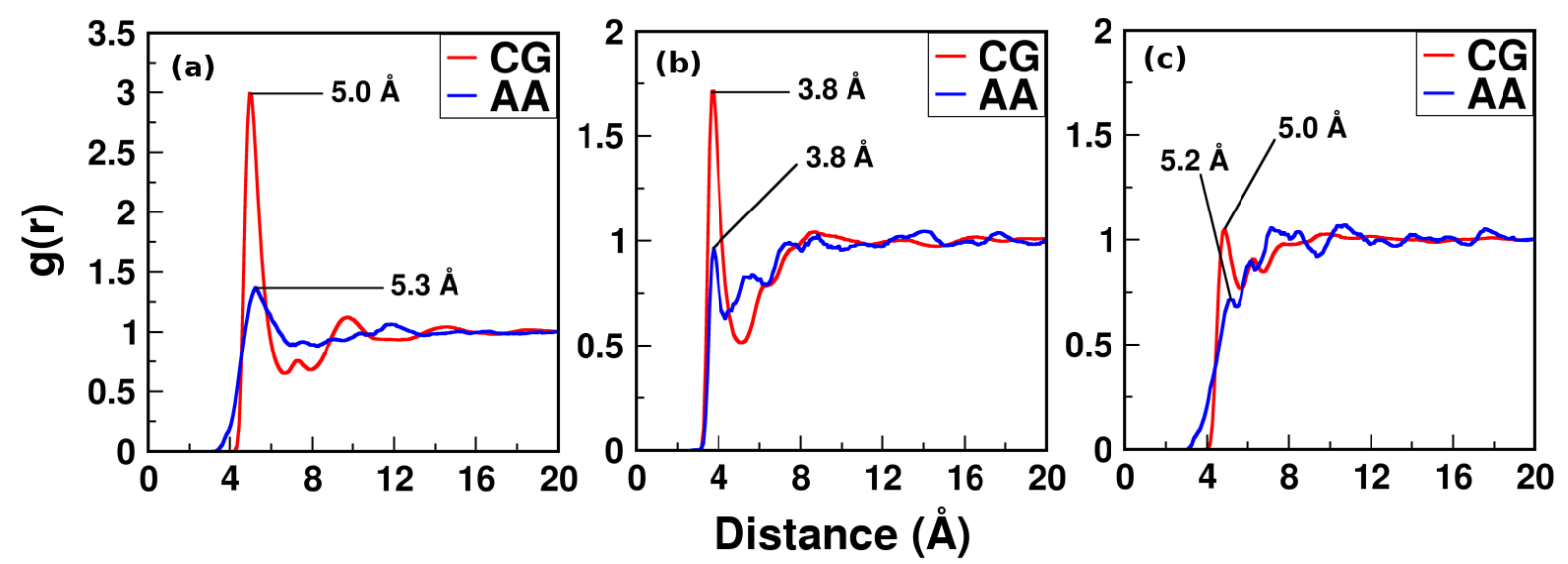

Figure C19.6: RDFs of (a) TOL4, (b) PL1, and (c) TL2W

Analogue 1: Pyrrole

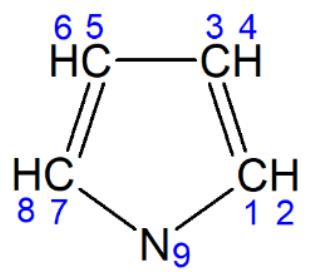

$\mathrm{H}_{10}$

Figure C19.7: All-atom pyrrole with atom numbering

PL2

PL1

Figure C19.8: CG model of pyrrole showing bead types

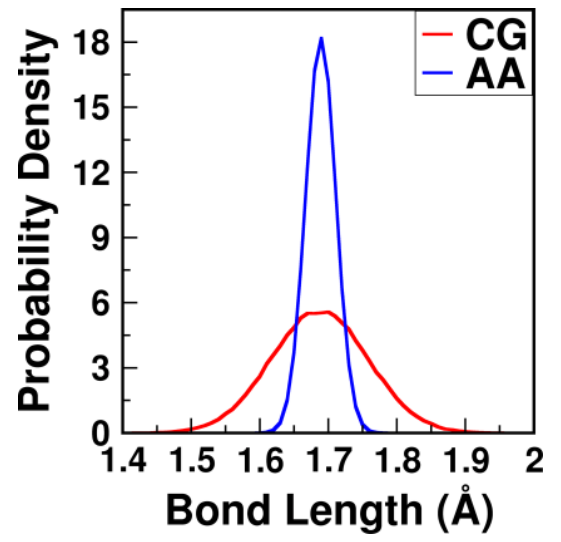

Figure C19.9: Distribution of bond PL1-PL2 


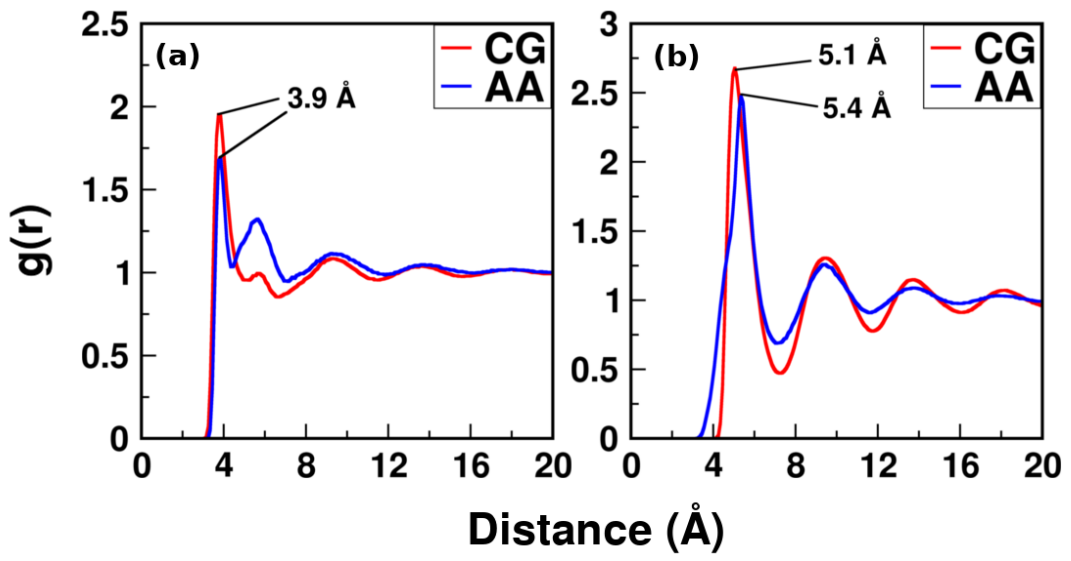

Figure C19.10: RDFs of (a) PL1 and (b) PL2

Analogue 2: Toluene

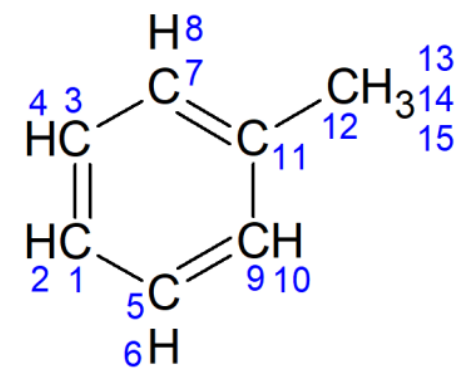

Figure C19.11: All-atom toluene with atom numbering

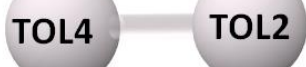

Figure C19.12: CG model of toluene showing bead types

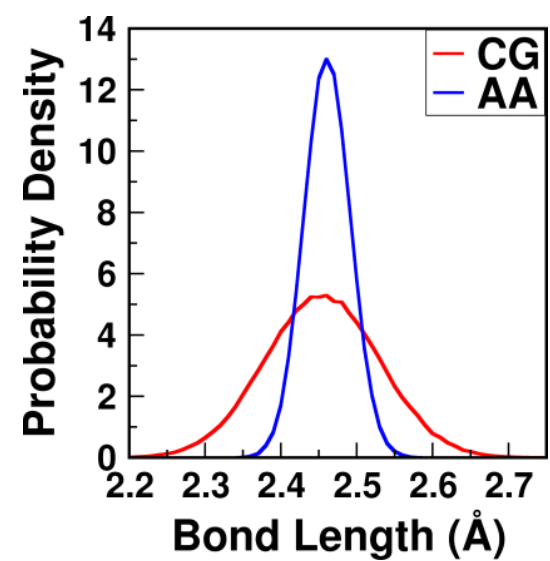

Figure C19.13: Distribution of bond TOL4-TOL2 


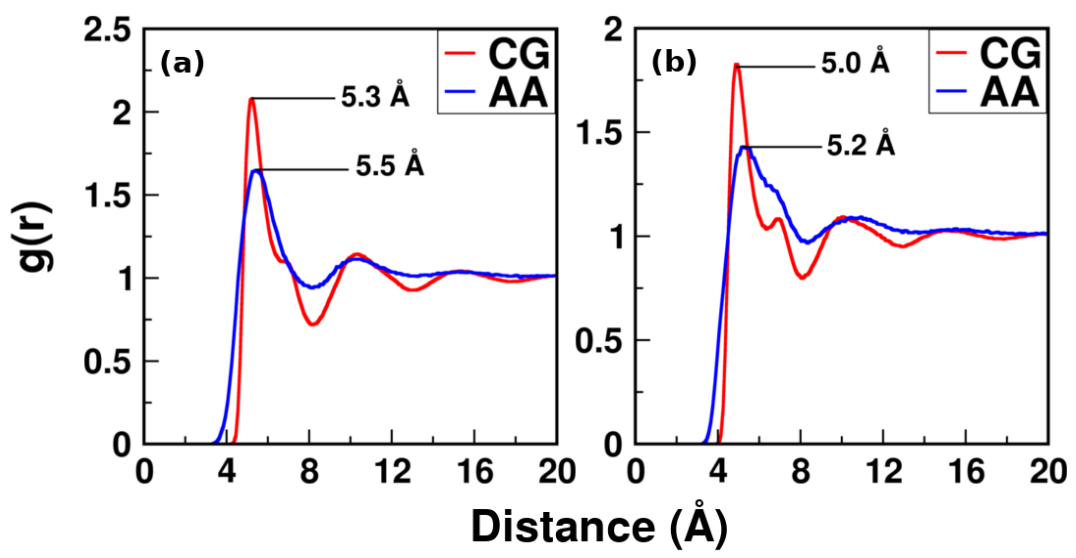

Figure C19.14: RDFs of (a) TOL4 and (b) TOL2 


\section{C20: Tyrosine}

\section{Tyr-Tyr Dipeptide}

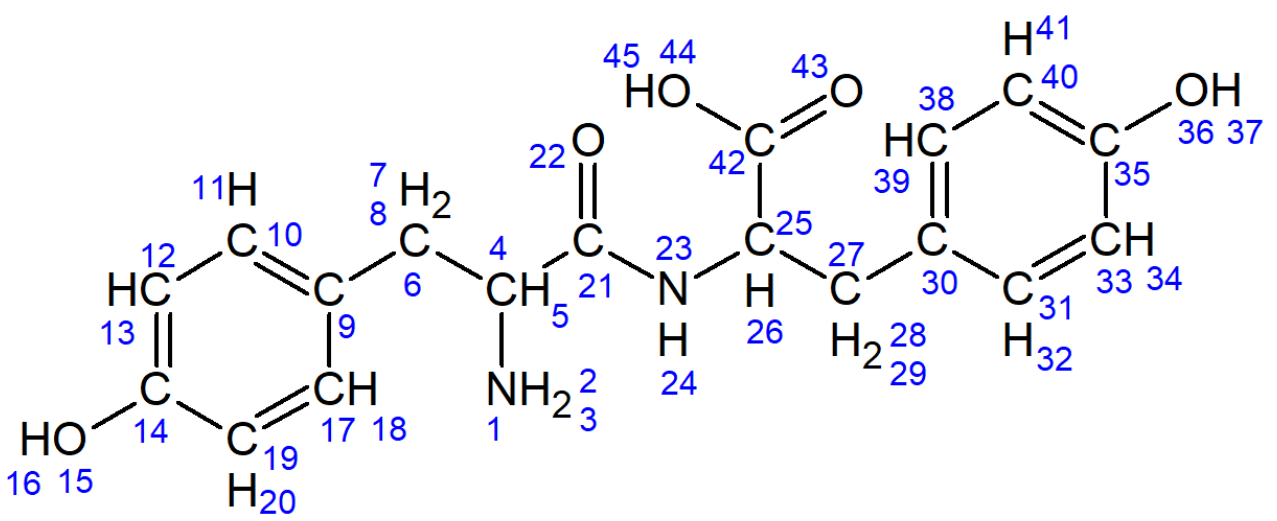

Figure C20.1: All-atom Tyrosine dipeptide with atom numbering

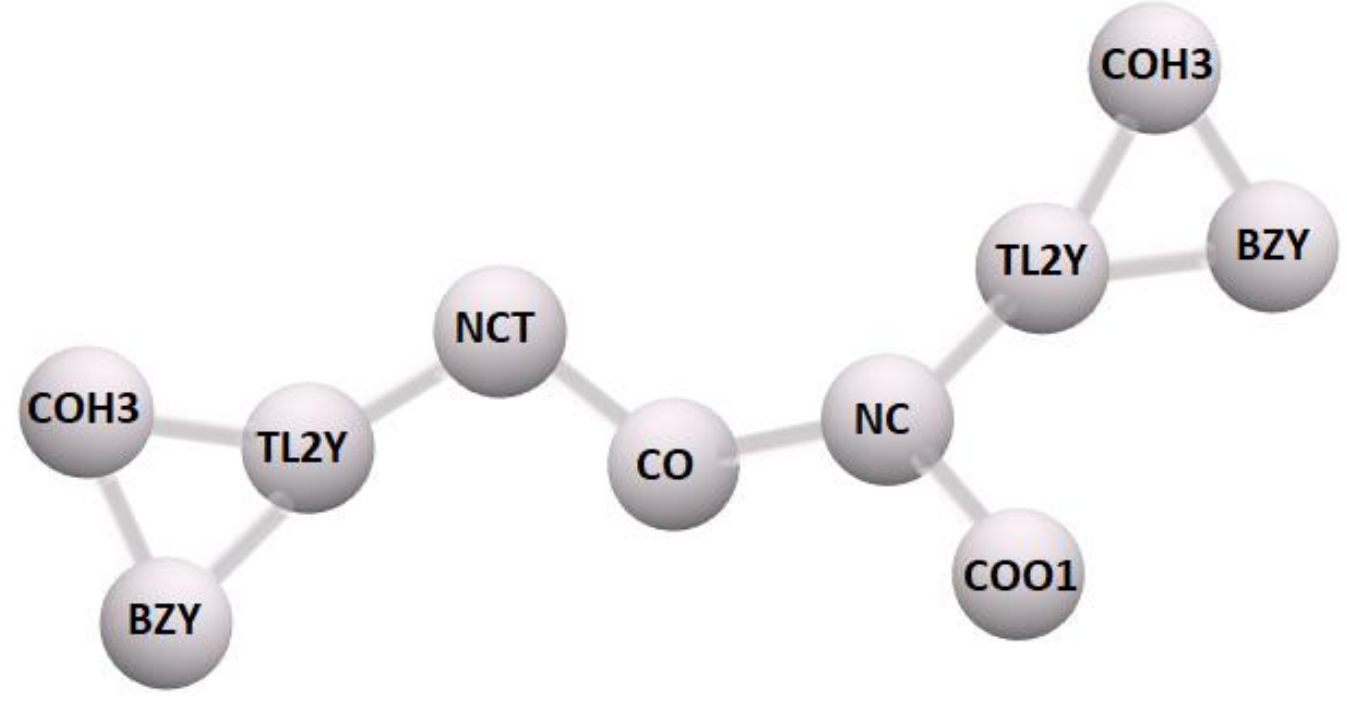

Figure C20.2: CG model of Tyrosine dipeptide showing bead types 

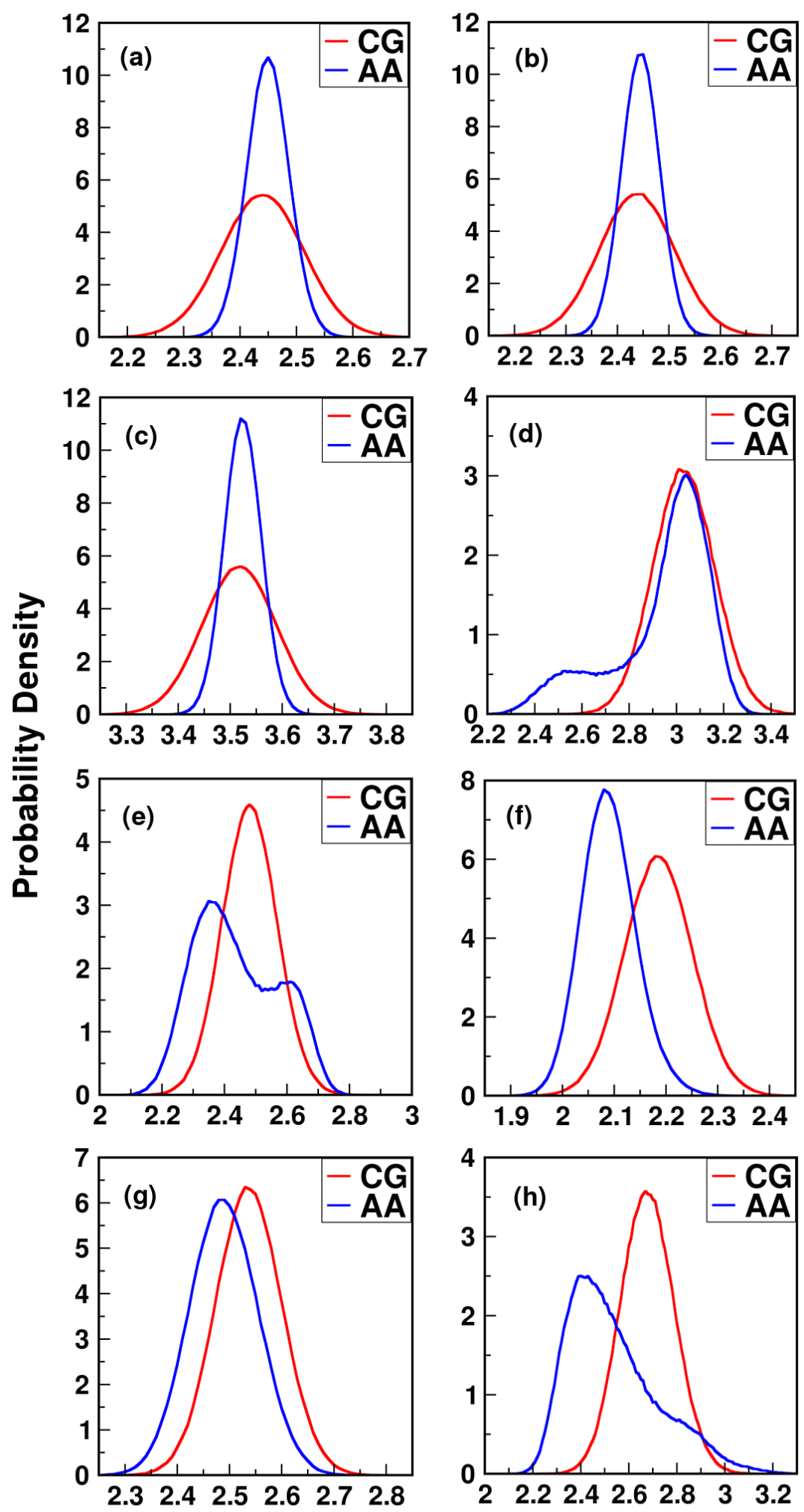

\section{Bond Length (Å)}

Figure C20.3: Distributions of bonds (a) TL2Y-BZY, (b) BZY-COH3, (c) COH3-TL2Y, (d) TL2Y-NCT, (e) NCT-CO, (f) CO-NC, (g) NC-COO1, and (h) NC-TL2Y 

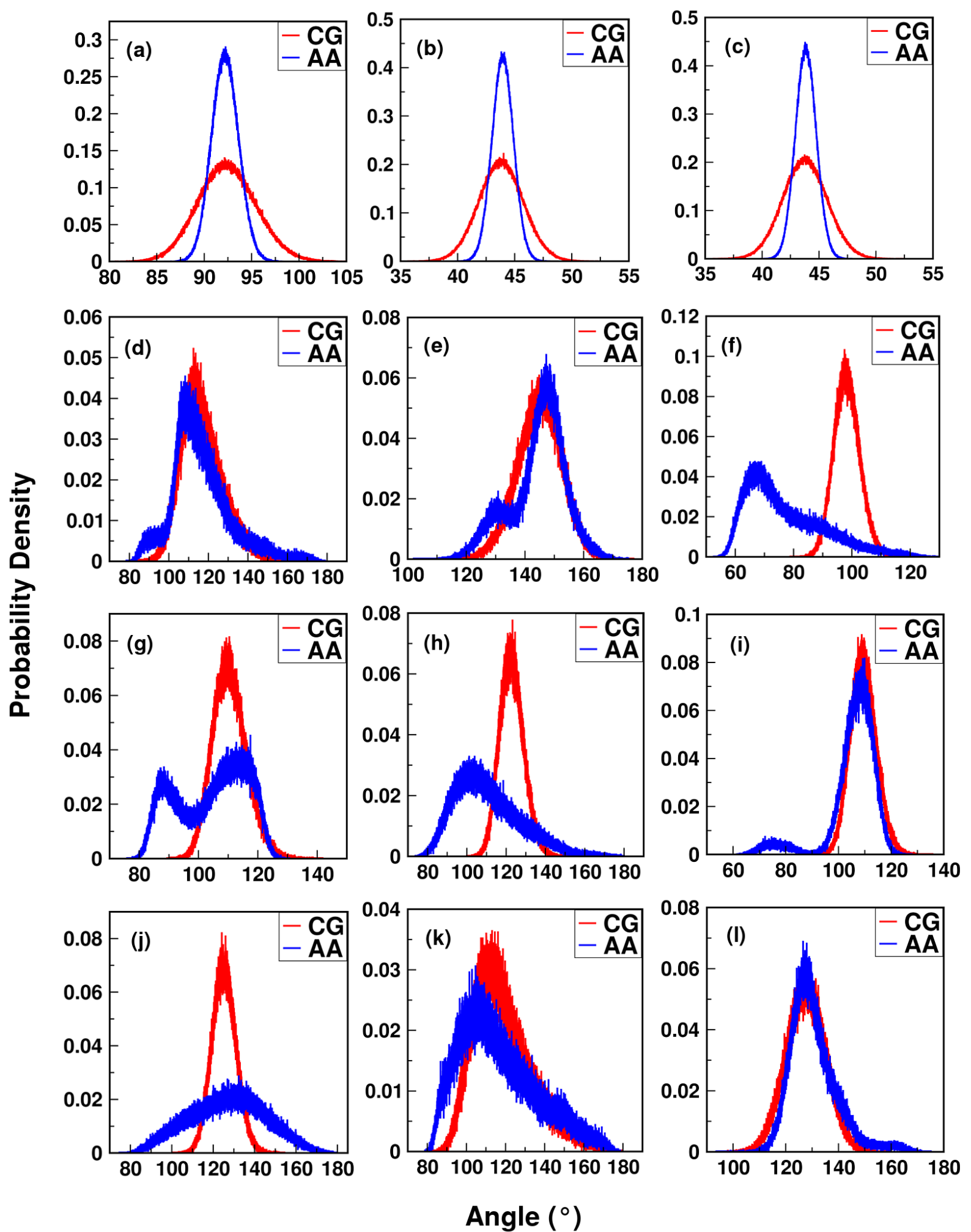

Figure C20.4: Distributions of angles (a) TL2Y-BZY-COH3, (b) BZY-YS3-TL2Y, (c) COH3-TL2Y-BZY, (d) BZY-TL2Y-NCT, (e) COH3-TL2Y-NCT, (f) TL2Y-NCT-CO, (g) NCT-CO-NC, (h) CO-NC-COO1, (i) TL2YNC-COO1, (j) CO-NC-TL2Y, (k) BZY-TL2Y-NC, and (l) COH3-TL2Y-NC 

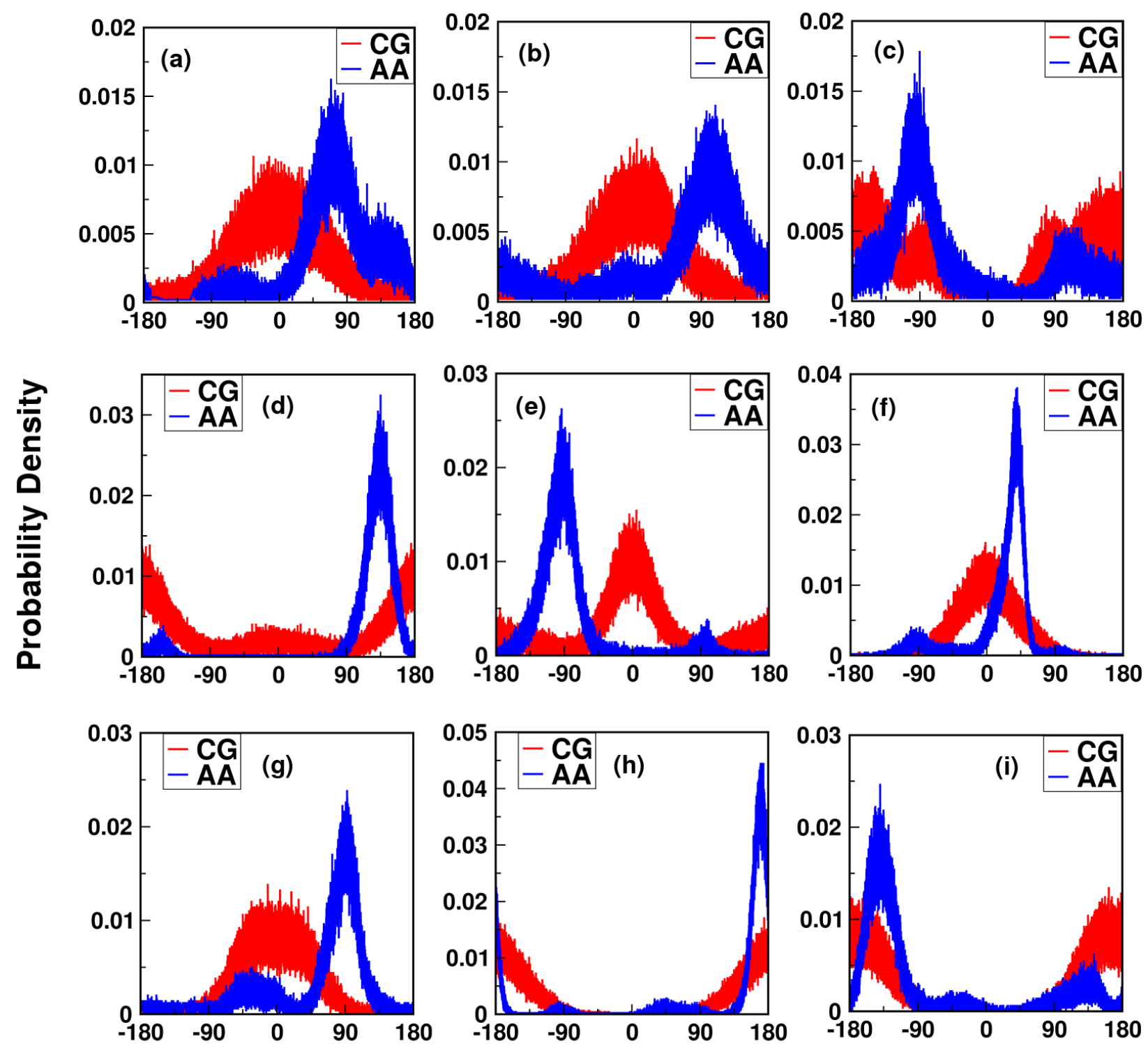

\section{Dihedral $\left({ }^{\circ}\right)$}

Figure C20.5: Distributions of dihedrals (a) COH3-TL2Y-NCT-CO, (b) BZ-TL2Y-NCT-CO, (c) TL2Y-NCTCO-NC, (d) NCT-CO-NC-COO1, (e) NCT-CO-NC-TL2Y, (f) CO-NC-TL2Y-COH3, (g) CO-NC-TL2Y-BZ, (h) COH3-TL2Y-NC-COO1, and (i) BZ-TL2Y-NC-COO1 

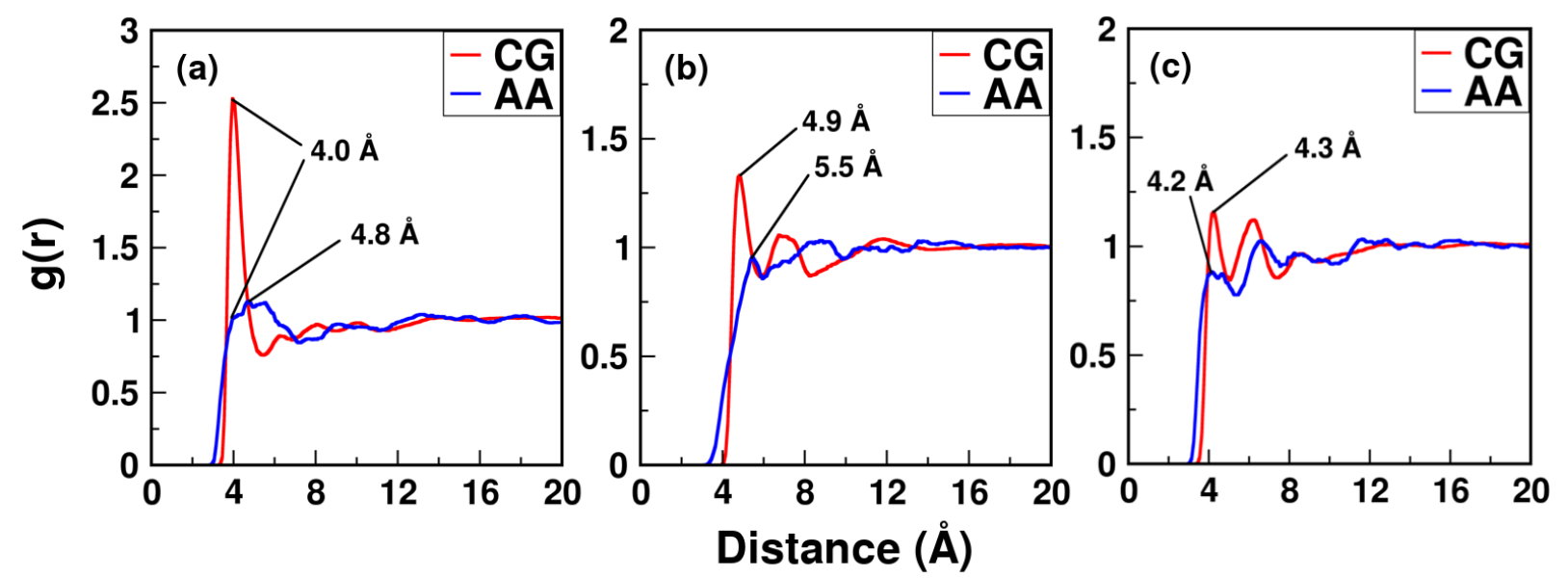

Figure C20.6: RDFs of (a) $\mathrm{COH} 3$, (b) TL2Y, and (c) BZY

\section{Analogue: m-Cresol}

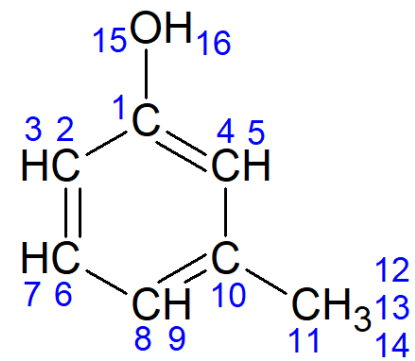

Figure C20.7: All-atom $\mathrm{m}$-Cresol with atom numbering

\section{$\mathrm{COH} 3$}

\section{BZY}

\section{TL2Y}

Figure C20.8: CG model of m-Cresol showing bead types
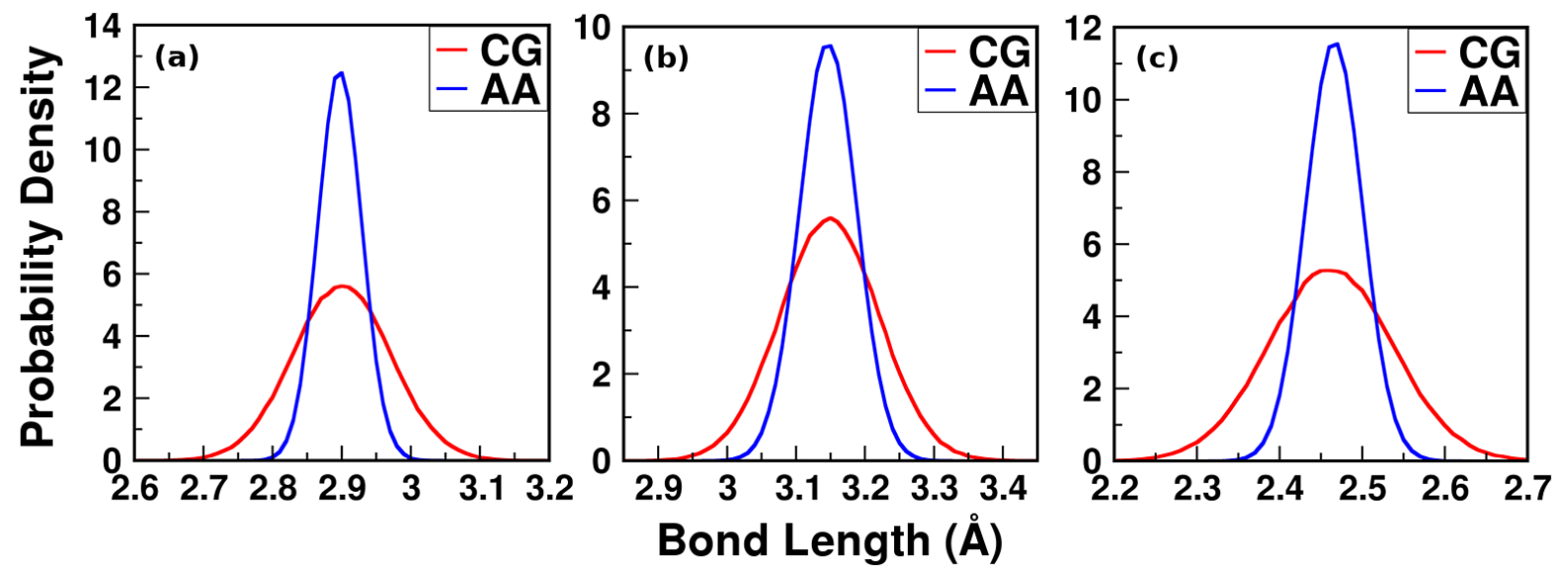

Figure C20.9: Distributions of bonds (a) COH3-BZY, (b) COH3-TL2Y, and (c) BZY-TL2Y 

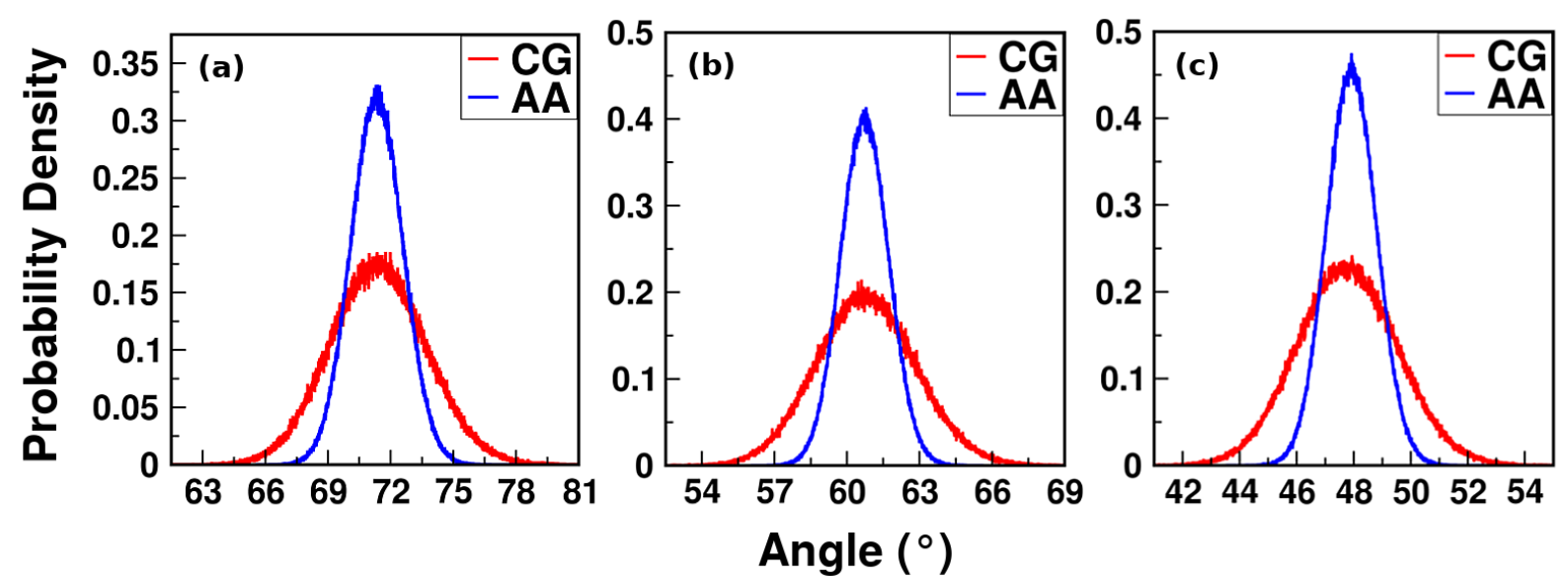

Figure C20.10: Distributions of angles (a) COH3-BZY-TL2Y, (b) COH3-TL2Y-BZY, and (c) BZY-COH3TL2Y
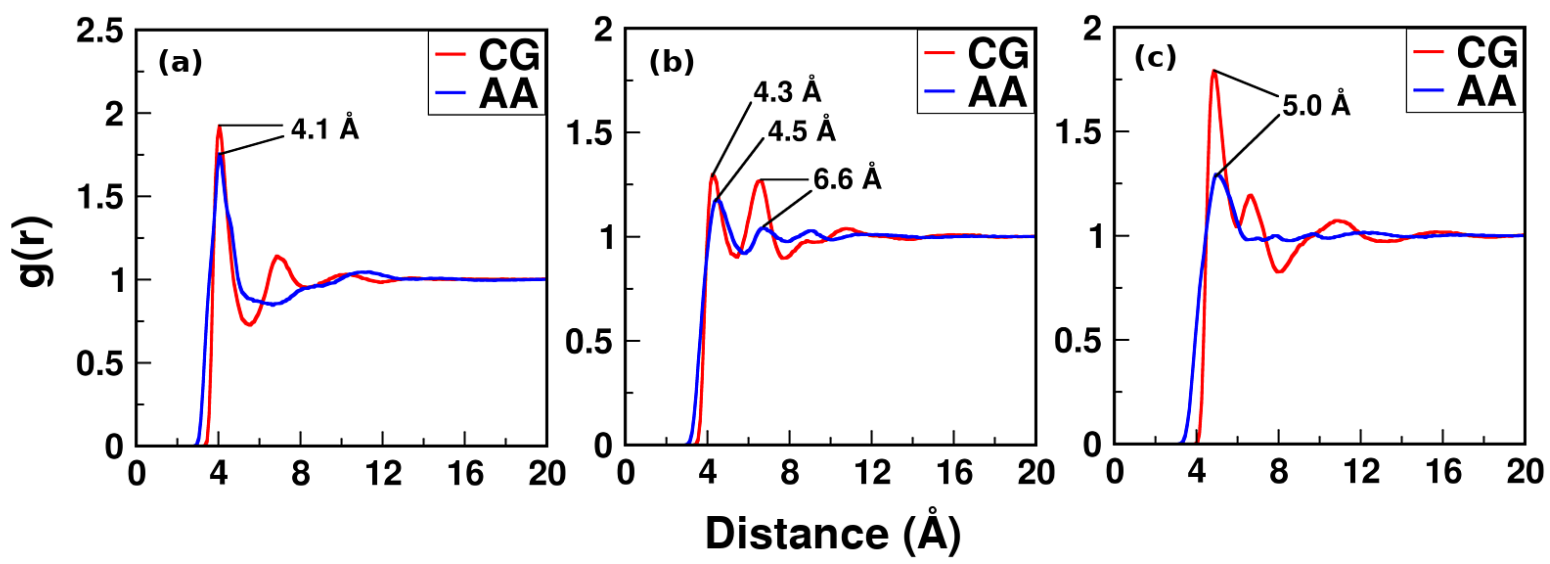

Figure C20.11: RDFs of (a) $\mathrm{COH} 3$, (b) BZY, and (c) TL2Y 


\section{C21: Valine}

\section{Val-Val Dipeptide}

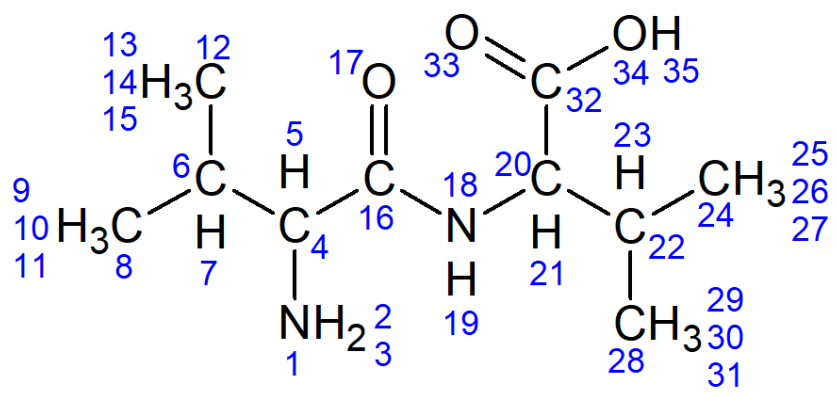

Figure C21.1: All-atom Valine dipeptide with atom numbering

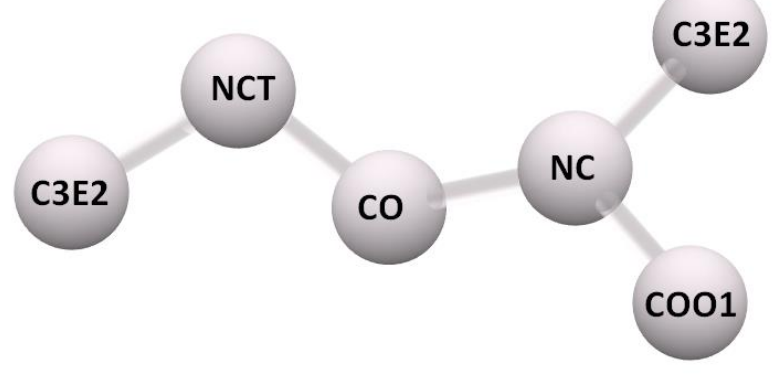

Figure C21.2: CG model of valine dipeptide showing bead types 

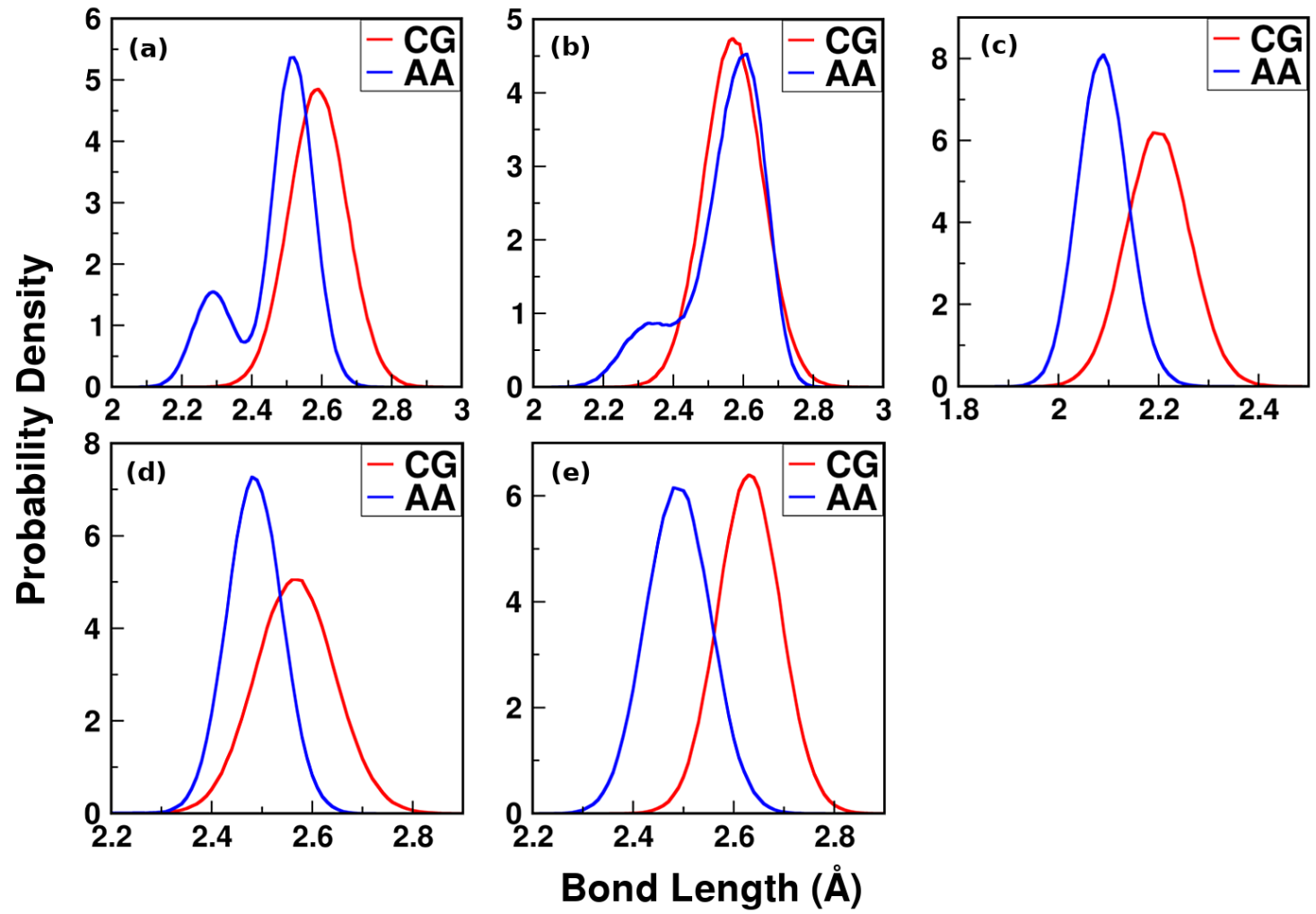

Figure C21.3: Distributions of bonds (a) C3E2-NCT, (b) NCT-CO, (c) CO-NC, (d) NC-C3E2, and (e) NC$\mathrm{COO1}$ 

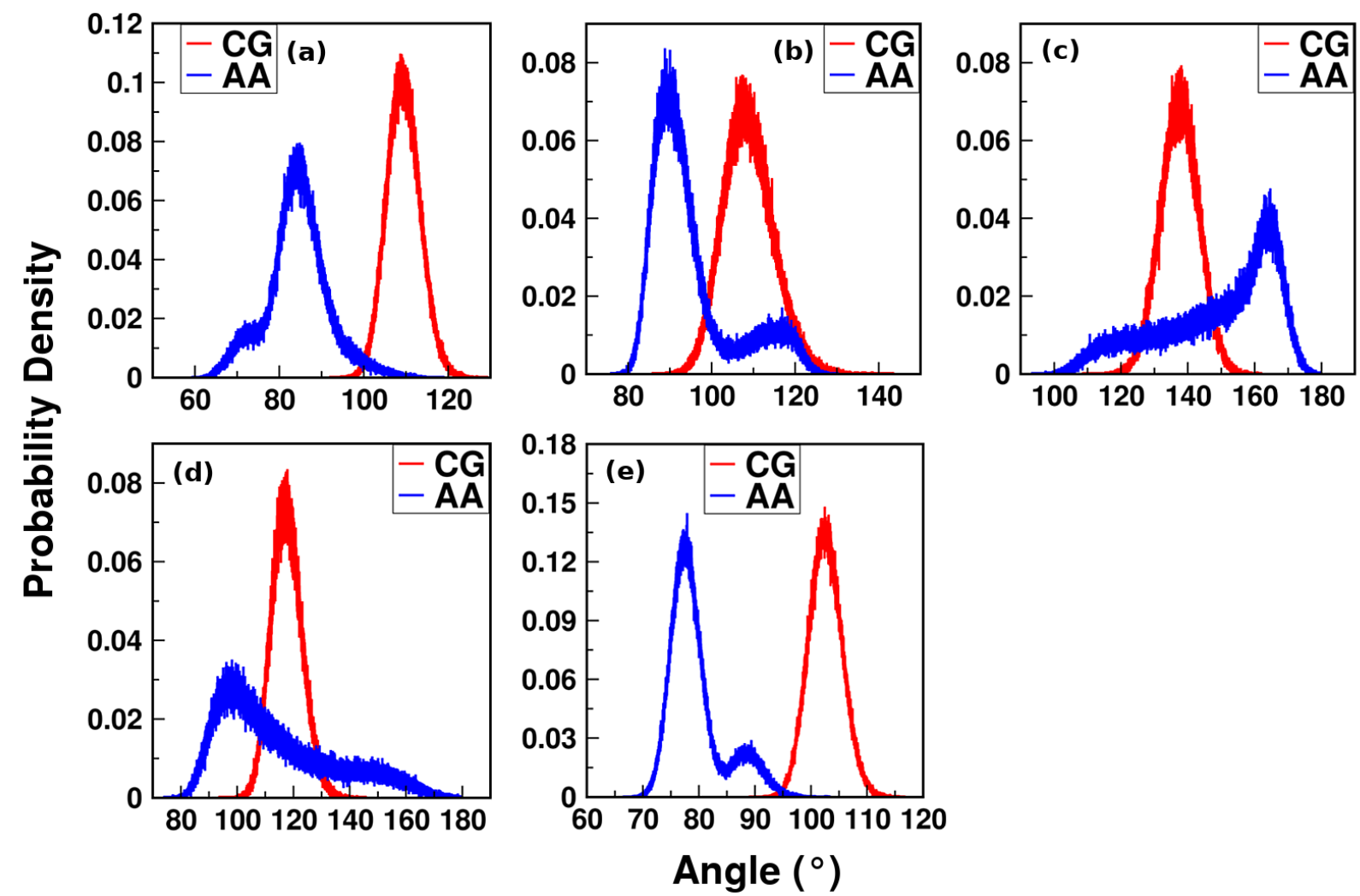

Figure C21.4: Distribution of angles (a) C3E2-NCT-CO (b) NCT-CO-NC, (c) CO-NC-C3E2, (d) CO-NCCOO1, and (e) C3E2-NC-COO1
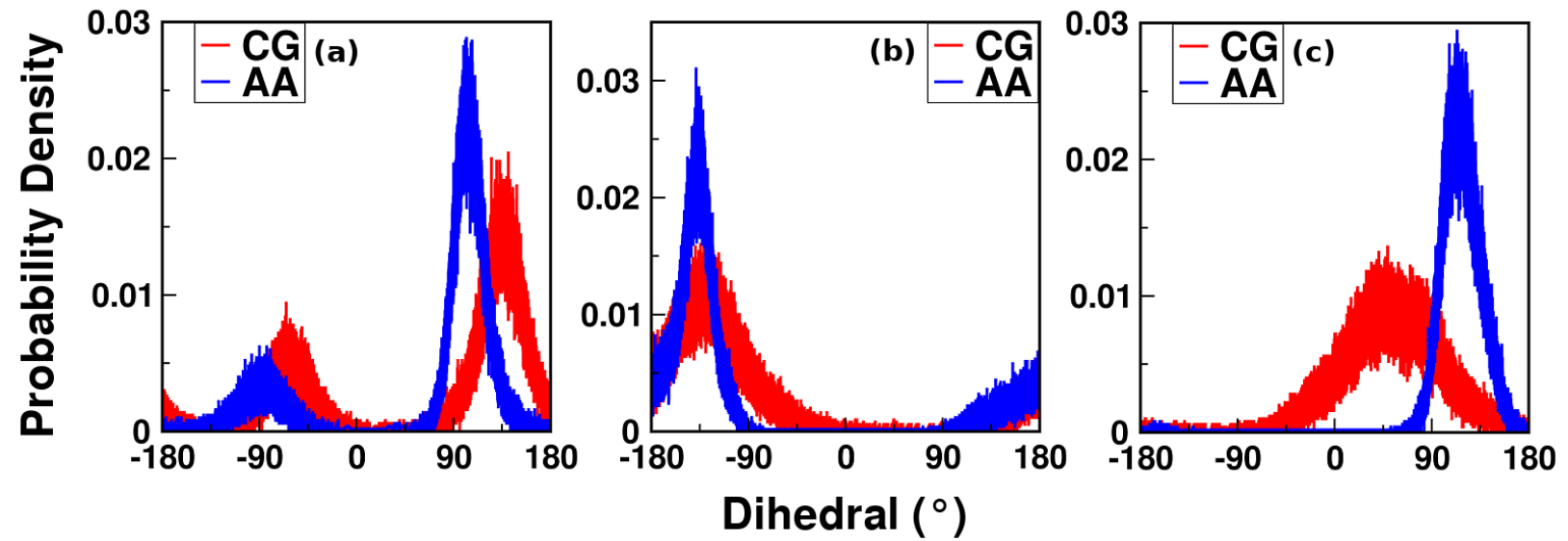

Figure C21.5: Distribution of dihedrals (a) C3E2-NCT-CO-NC, (b) NCT-CO-NC-C3E2, and (c) NCT-CONC-COO1 


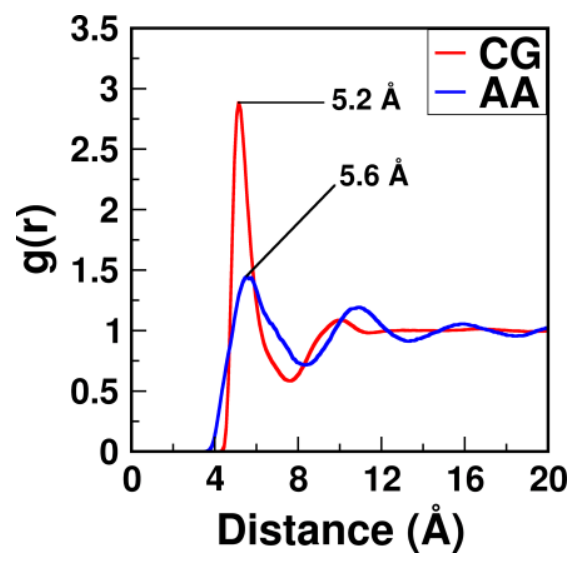

Figure C21.6: RDF of C3E2

\section{Analogue: 2,3-dimethylbutane}

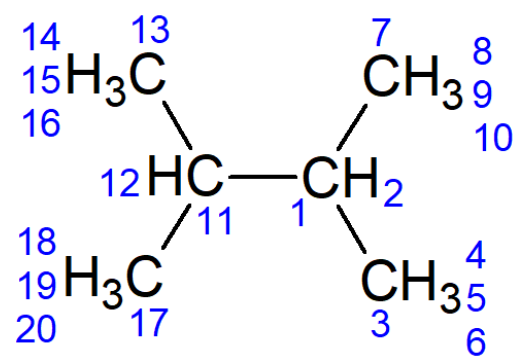

Figure C21.7: All-atom 2,3-dimethylbutane with atom numbering

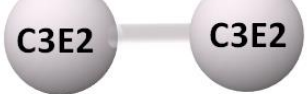

Figure C21.8: CG model of 2,3-dimethylbutane showing bead types

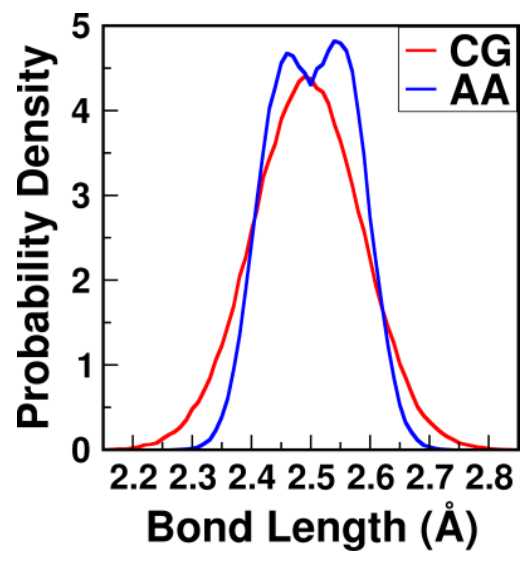

Figure C21.9: Distribution of bond C3E2-C3E2 


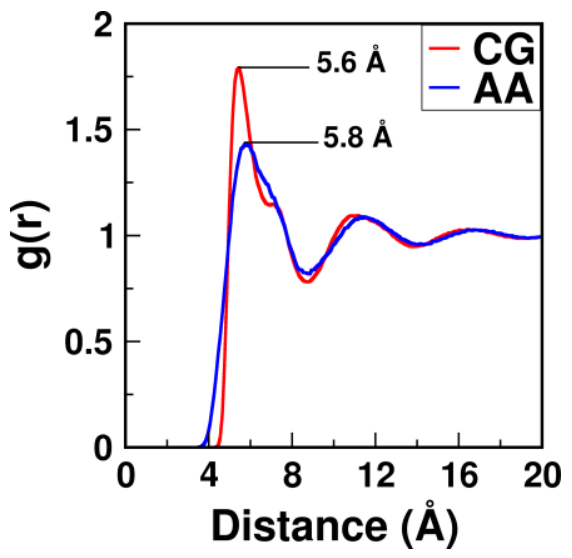

Figure C21.10: RDF of C3E2

\section{Val-Val-Val Tripeptide}

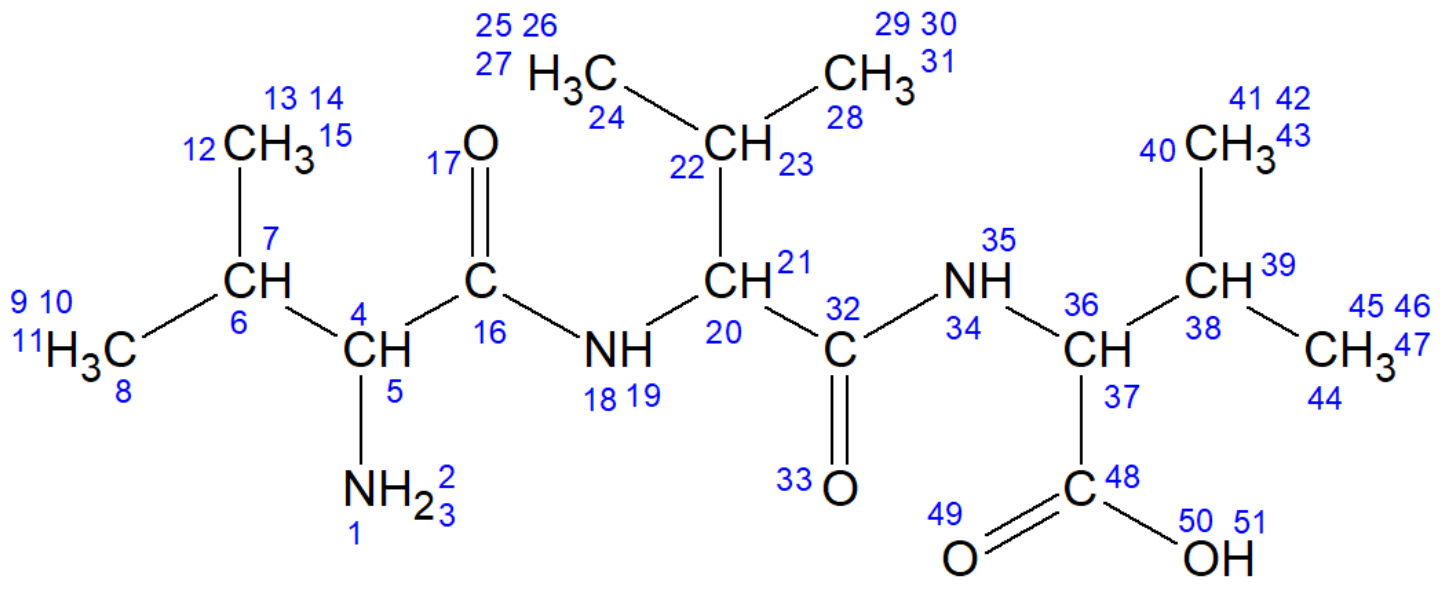

Figure C21.11: All-atom valine tripeptide with atom numbering

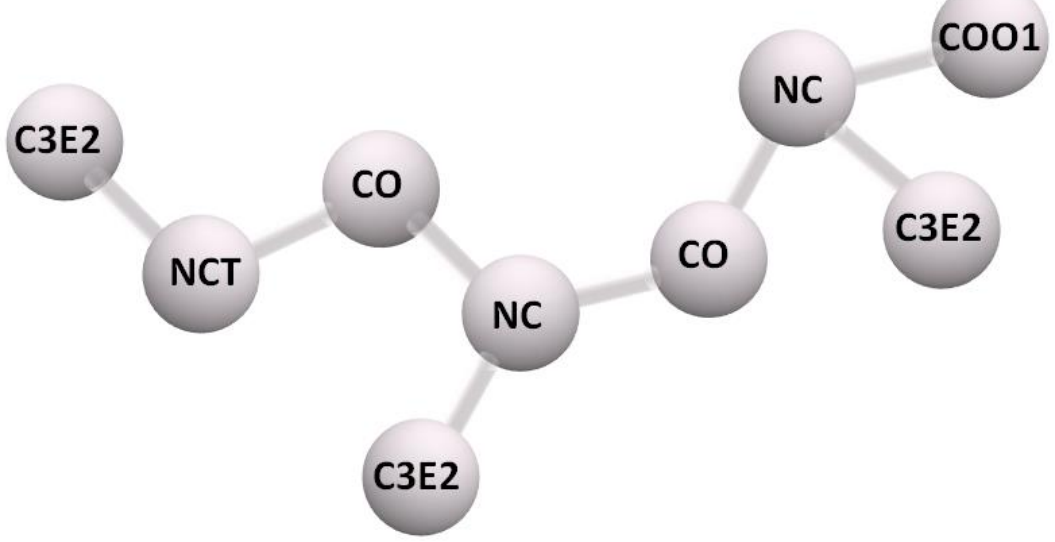

Figure C21.12: CG model of valine tripeptide showing bead types 


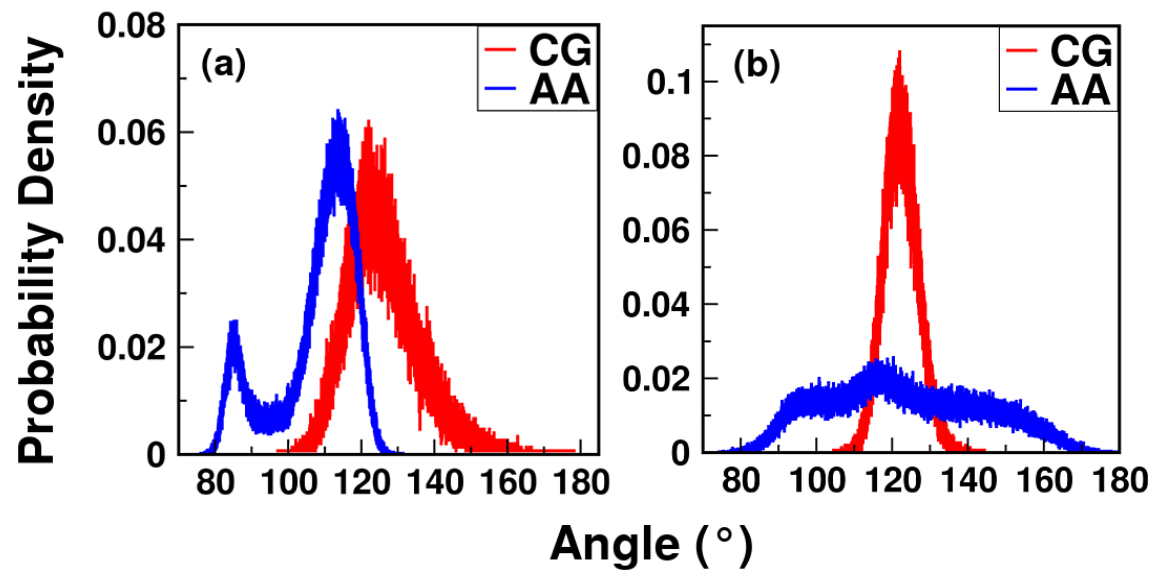

Figure C21.11: Angle distributions of (a) NC-CO-NC and (b) CO-NC-CO.
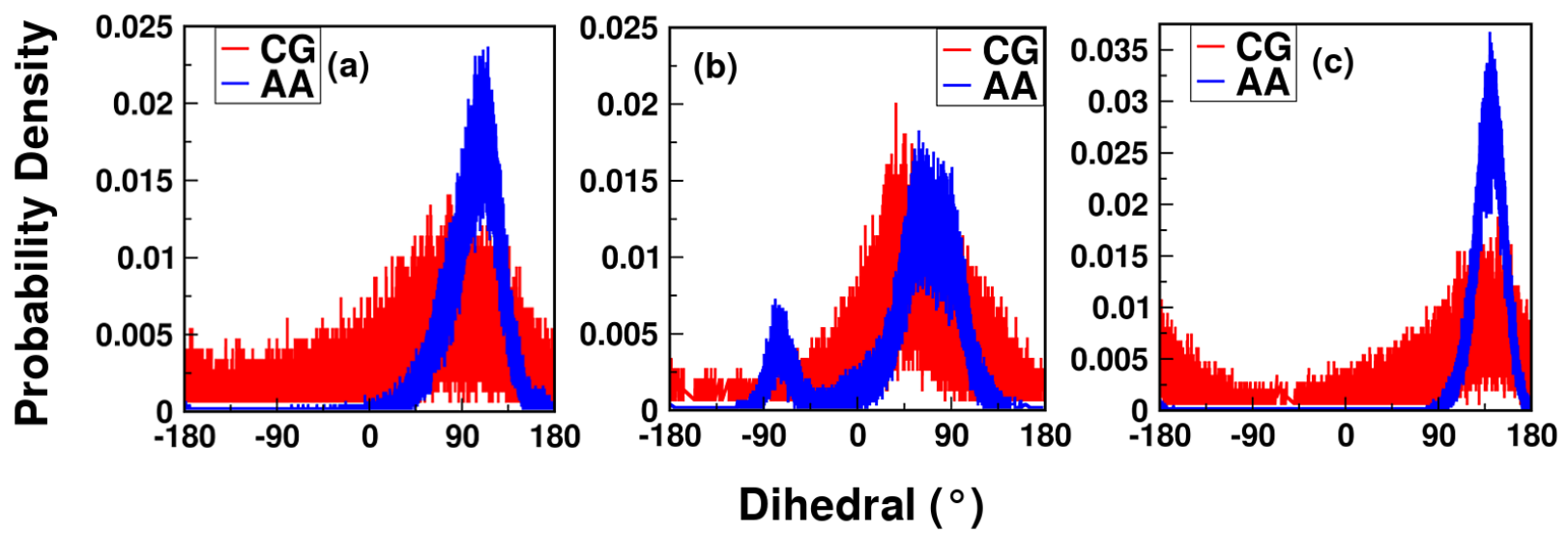

Figure C21.12: Dihedral distributions of (a) NT-CO-NC-CO, (b) CO-NC-CO-NC, and (c) NC-CO-NC-CT. 


\section{Appendix D: Topology, Parameter, and Configuration Files}

\section{D1: Topology File}

!! Coarse-Grained Topology File for amino acids/peptides !!

\begin{tabular}{|c|c|}
\hline MASS -1 C2E & 29.07 \\
\hline MASS -1 C $2 M$ & 28.06 \\
\hline MASS - 1 C3E & 43.10 \\
\hline MASS -1 C $3 M$ & 42.09 \\
\hline MASS -1 CCR1 & 26.04 ! His \\
\hline MASS -1 NCR1 & 28.04 ! His \\
\hline MASS -1 NCR2 & 27.03 ! His \\
\hline MASS -1 NCC1 & 44.09! Ala N-terminus \\
\hline MASS -1 NCC2 & 43.08 ! Ala backbone \\
\hline MASS -1 C41 & 57.13 ! Leu \\
\hline MASS -1 CON2 & $58.07 !$ Asn \\
\hline MASS -1 COO2 & $59.05 !$ Asp \\
\hline MASS -1 RS1 & 28.06 ! Arginine side chain bead \\
\hline MASS -1 RS2 & 29.05 ! Arginine side chain bead \\
\hline MASS - 1 RS3 & 43.06 ! Arginine side chain bead \\
\hline MASS -1 SC & 47.10 ! Cys \\
\hline MASS -1 CON1 & 44.04 ! Glutamine side chain outer \\
\hline MASS -1 GNT & 30.06 ! Glycine N-terminus \\
\hline MASS - 1 GNC & 29.05 ! Glycine backbone \\
\hline MASS -1 C2E2 & 28.06 ! Ile side chain inner \\
\hline MASS -1 CS & 47.10! Methionine side chain outer \\
\hline MASS -1 BZF2 & 26.04 \\
\hline MASS -1 BZF1 & 26.04 \\
\hline MASS - 1 TL2F & 39.06 ! Phenylalanine toluene bead \\
\hline MASS -1 TL2W & 39.06 ! Tryptophan toluene bead \\
\hline MASS -1 TL2Y & 39.06 ! Tyrosine toluene bead \\
\hline MASS -1 PNC & 27.03 ! Proline N-C bead \\
\hline MASS -1 PS & 42.09! Proline 3-C side chain bead \\
\hline MASS -1 COH1 & 31.04 ! Serine side chain bead \\
\hline MASS $-1 \mathrm{CCOH}$ & 45.07! Threonine side chain \\
\hline MASS -1 TOL4 & $52.08 ! \operatorname{Trp}$ \\
\hline MASS -1 PL1 & $40.05 ! \operatorname{Trp}$ \\
\hline MASS - 1 COH3 & 42.04 \\
\hline MASS -1 BZY & 26.04 \\
\hline MASS -1 C3E2 & 43.10 \\
\hline MASS -1 CO & 28.01 ! backbone carbonyl \\
\hline MASS -1 NC & 28.04 ! backbone N-C \\
\hline
\end{tabular}




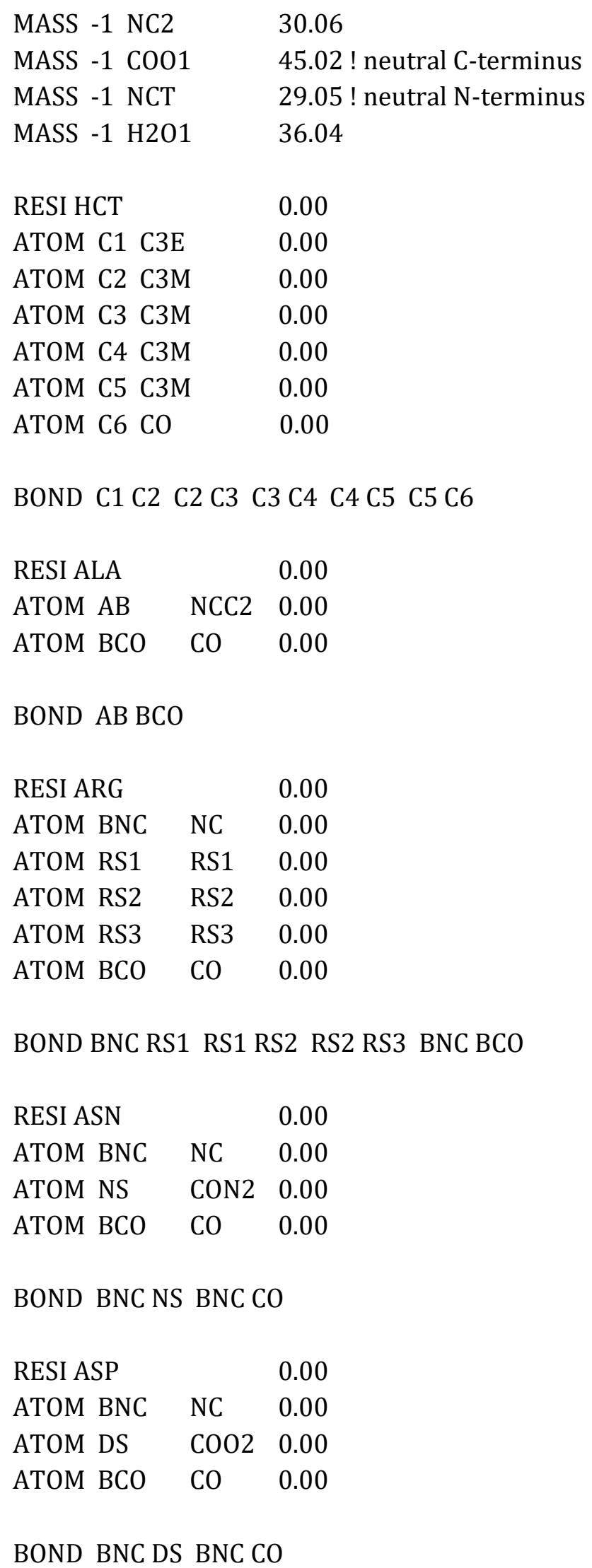




$\begin{array}{lll}\text { RESI CYS } & & 0.00 \\ \text { ATOM BNC } & \text { NC } & 0.00 \\ \text { ATOM CS } & \text { SC } & 0.00 \\ \text { ATOM BCO } & \text { CO } & 0.00 \\ & & \\ \text { BOND BNC CS BNC CO } \\ \text { RESI GLU } & & 0.00 \\ \text { ATOM BNC } & \text { NC } & 0.00 \\ \text { ATOM ES1 } & \text { C2M } & 0.00 \\ \text { ATOM ES2 } & \text { COO1 } & 0.00 \\ \text { ATOM BCO } & \text { CO } & 0.00 \\ \text { BOND BNC ES1 ES1 ES2 BNC BCO } \\ \text { RESI GLN } & & \\ \text { ATOM BNC } & \text { NC } & 0.00 \\ \text { ATOM BNC } & \text { NC } & 0.00 \\ \text { ATOM IS2 } & \text { C2E } & 0.00 \\ \text { ATOM BCO } & \text { CO } & 0.00 \\ \text { ATOM QS2 } & \text { C2M } & 0.00 \\ \text { ATOM BCO } & \text { CO } & 0.00 \\ \text { ATOM BNC HS1 HS1 HS2 HS1 HS3 HS2 HS3 BNC BCO } \\ \text { BTON }\end{array}$


BOND BNC IS1 IS1 IS2 BNC BCO

\begin{tabular}{|c|c|c|}
\hline RESI LEU & & 0.00 \\
\hline ATOM BNC & $\mathrm{NC}$ & 0.00 \\
\hline ATOM LS & $\mathrm{C} 41$ & 0.00 \\
\hline ATOM BCO & $\mathrm{CO}$ & 0.00 \\
\hline BOND BNC L & BNC E & \\
\hline RESI LYS & & 0.00 \\
\hline ATOM BNC & NC & 0.00 \\
\hline ATOM KS1 & C3M & 0.00 \\
\hline ATOM KS2 & NC2 & 0.00 \\
\hline ATOM BCO & $\mathrm{CO}$ & 0.00 \\
\hline BOND BNC K & $1 \mathrm{KS} 1$ & KS2 BNC BCO \\
\hline RESI MET & & 0.00 \\
\hline ATOM BNC & NC & 0.00 \\
\hline ATOM MS1 & $\mathrm{C} 2 \mathrm{M}$ & 0.00 \\
\hline ATOM MS2 & CS & 0.00 \\
\hline BOND BNC M & 1 MS1 & MS2 BNC BCO \\
\hline RESI PHE & & 0.00 \\
\hline ATOM BNC & NC & 0.00 \\
\hline ATOM FS1 & TL2F & 0.00 \\
\hline ATOM FS21 & BZF1 & 0.00 \\
\hline ATOM FS22 & BZF2 & 0.00 \\
\hline ATOM BCO & $\mathrm{CO}$ & 0.00 \\
\hline BOND BNC F & 1 FS1 & S21 FS1 FS22 FS21 FS22 BNC BCO \\
\hline RESI PRO & & 0.00 \\
\hline ATOM PNC & PNC & 0.00 \\
\hline ATOM PS & PS & 0.00 \\
\hline ATOM BCO & $\mathrm{CO}$ & 0.00 \\
\hline BOND PNC P & PNC B & \\
\hline RESI SER & & 0.00 \\
\hline ATOM BNC & NC & 0.00 \\
\hline
\end{tabular}




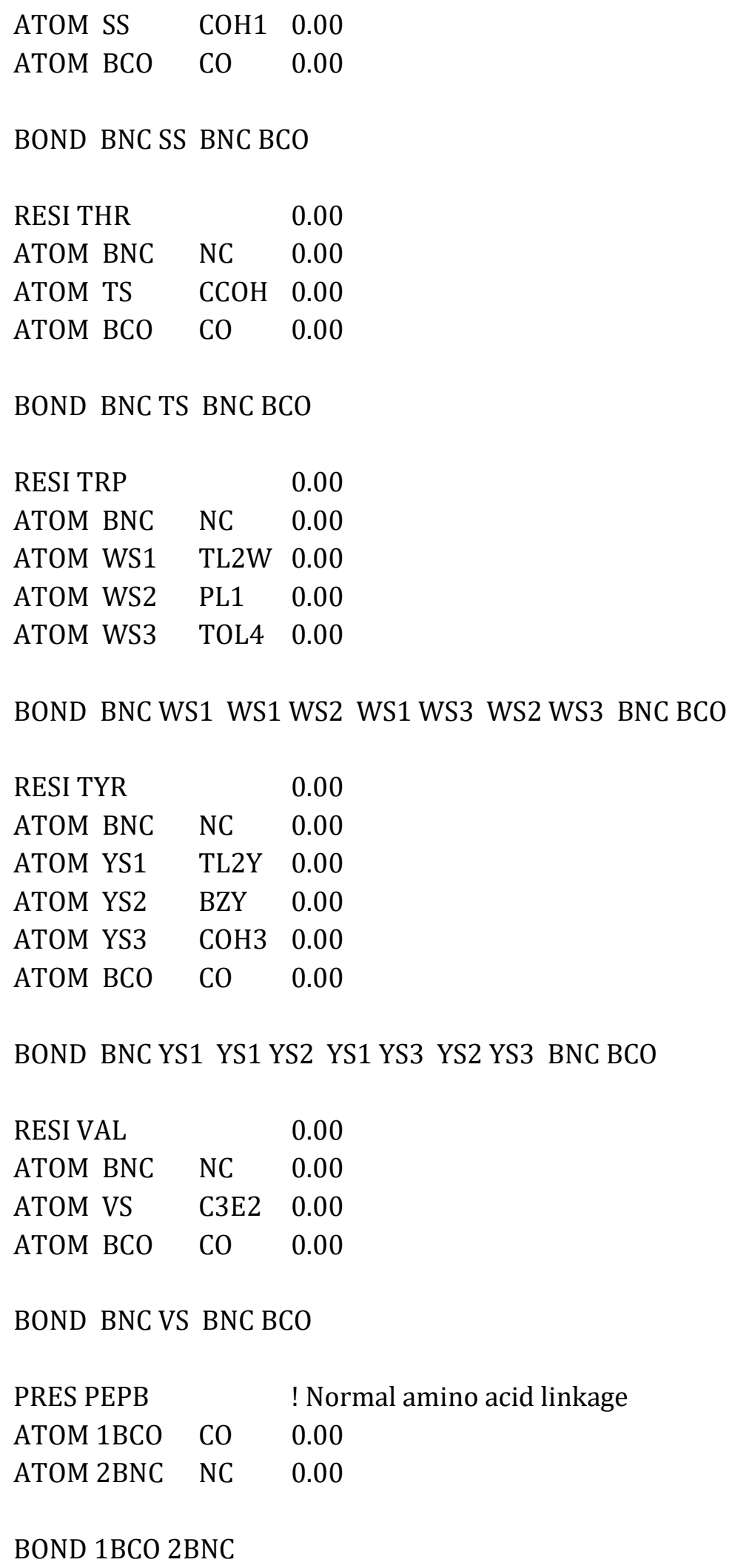




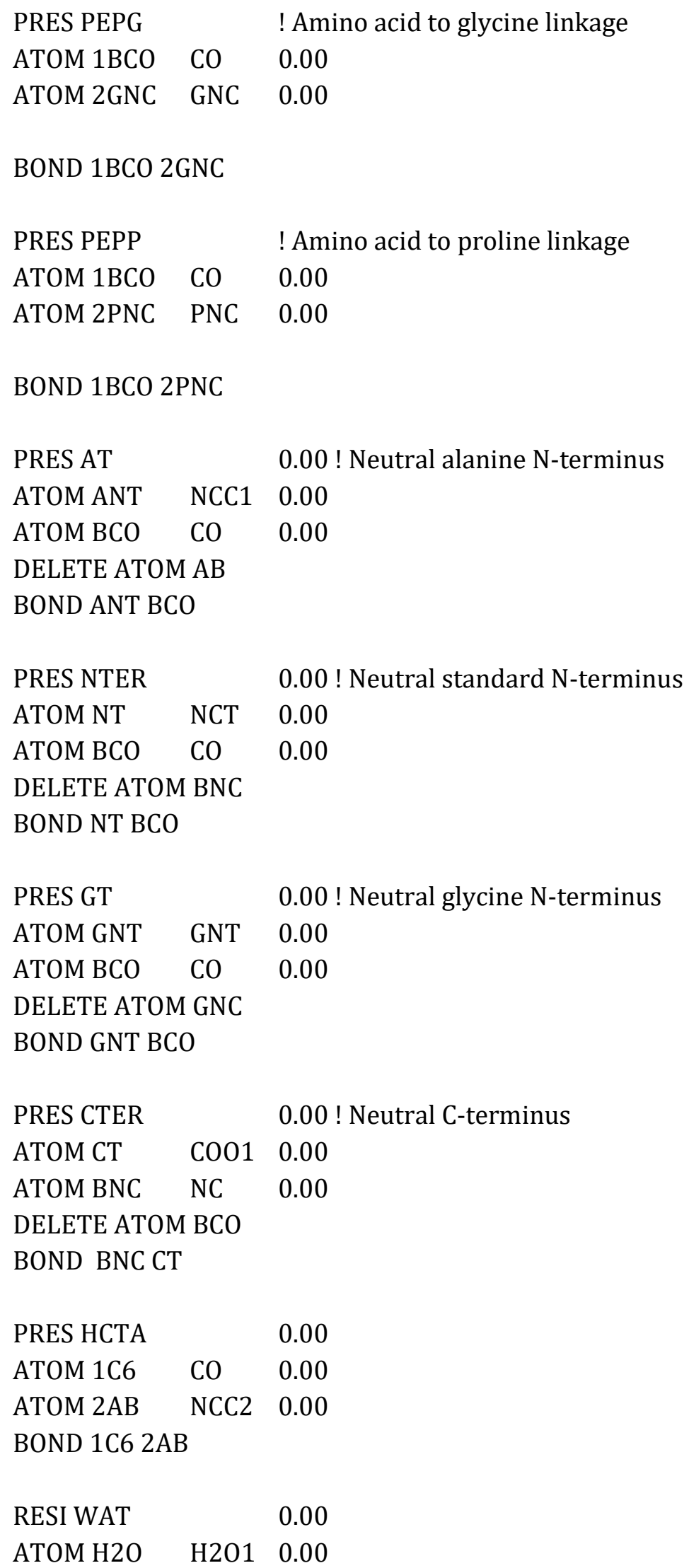




\section{D2: Parameter File}

!! Coarse-Grained Parameter File for the amino acids and peptides !!

!! This is for use with "exclude 1-2" ONLY. !!

!! Only neutral amino acids and neutral termini are represented. !!

\begin{tabular}{|c|c|}
\hline ATOMS & \\
\hline MASS - 1 C2E & 29.07 \\
\hline MASS - 1 C $2 M$ & 28.06 \\
\hline MASS -1 C3E & 43.10 \\
\hline MASS -1 C3M & 42.09 \\
\hline MASS -1 CCR1 & 26.04 ! His \\
\hline MASS -1 NCR1 & 28.04 ! His \\
\hline MASS -1 NCR2 & 27.03 ! His \\
\hline MASS -1 NCC1 & 44.09 ! Ala N-terminus \\
\hline MASS -1 NCC2 & 43.08 ! Ala backbone \\
\hline MASS -1 C41 & $57.13 ! \mathrm{Leu}$ \\
\hline MASS -1 CON2 & $58.07 !$ Asn \\
\hline MASS -1 COO2 & $59.05 !$ Asp \\
\hline MASS -1 RS1 & 28.06 ! Arginine side chain bead \\
\hline MASS -1 RS2 & 29.05 ! Arginine side chain bead \\
\hline MASS -1 RS3 & 43.06 ! Arginine side chain bead \\
\hline MASS -1 SC & $47.10 !$ Cys \\
\hline MASS - 1 CON1 & 44.04 ! Glutamine side chain outer \\
\hline MASS -1 GNT & 30.06 ! Glycine N-terminus \\
\hline MASS -1 GNC & 29.05 ! Glycine backbone \\
\hline MASS -1 C2E2 & 28.06 ! Ile side chain inner \\
\hline MASS - 1 CS & 47.10! Methionine side chain outer \\
\hline MASS -1 BZF2 & 26.04 \\
\hline MASS -1 BZF1 & 26.04 \\
\hline MASS -1 TL2F & 39.06 ! Phenylalanine toluene bead \\
\hline MASS -1 TL2W & 39.06 ! Tryptophan toluene bead \\
\hline MASS -1 TL2Y & 39.06 ! Tyrosine toluene bead \\
\hline MASS -1 PNC & 27.03 ! Proline N-C bead \\
\hline MASS - 1 PS & 42.09! Proline 3-C side chain bead \\
\hline MASS - 1 COH1 & 31.04 ! Serine side chain bead \\
\hline MASS $-1 \mathrm{CCOH}$ & 45.07! Threonine side chain \\
\hline MASS -1 TOL4 & $52.08 ! \operatorname{Trp}$ \\
\hline MASS -1 PL1 & 40.05 ! Trp \\
\hline MASS - 1 COH3 & 42.04 \\
\hline MASS -1 BZY & 26.04 \\
\hline MASS -1 C3E2 & 43.10 \\
\hline MASS -1 CO & 28.01 ! backbone carbonyl \\
\hline
\end{tabular}




$\begin{array}{lll}\text { MASS }-1 & \text { NC } & 28.04 \text { ! backbone N-C } \\ \text { MASS }-1 \text { NC2 } & 30.06 \\ \text { MASS }-1 \text { CO01 } & 45.02 \text { ! neutral C-terminus } \\ \text { MASS }-1 \text { NCT } & 29.05 \text { ! neutral N-terminus } \\ \text { MASS }-1 \text { H2O1 } & 36.04\end{array}$

\begin{tabular}{|c|c|c|c|}
\hline \multicolumn{4}{|c|}{ BONDS } \\
\hline \multicolumn{4}{|c|}{$!$} \\
\hline \multicolumn{4}{|c|}{$! \mathrm{V}($ bond $)=\mathrm{Kb}(\mathrm{r}-\mathrm{r} 0)^{* *} 2$} \\
\hline \multicolumn{4}{|c|}{ ! } \\
\hline \multicolumn{4}{|c|}{ !Kb: kcal/mole/A**2 } \\
\hline \multicolumn{4}{|c|}{ !r0: A } \\
\hline \multicolumn{4}{|l|}{ ! } \\
\hline \multicolumn{2}{|c|}{ !bead types } & $\mathrm{Kb}$ & r0 \\
\hline \multicolumn{4}{|c|}{ ! Alanine } \\
\hline NCC1 & $\mathrm{CO}$ & 15 & 2.57 \\
\hline $\mathrm{CO}$ & NCC2 & 10 & 2.63 \\
\hline NCC2 & C001 & 30 & 2.54 \\
\hline \multicolumn{4}{|c|}{ ! Asparagine } \\
\hline CON2 & NCT & 8 & 3.00 \\
\hline NC & CON2 & 8 & 2.75 \\
\hline \multicolumn{4}{|c|}{ ! Aspartic Acid } \\
\hline $\mathrm{COO} 2$ & NCT & 8 & 3.00 \\
\hline NC & $\mathrm{COO} 2$ & 55 & 2.71 \\
\hline \multicolumn{4}{|c|}{ ! Arginine } \\
\hline NC & RS1 & 10 & $2.30 ! \mathrm{RS} 1=\mathrm{C} 2 \mathrm{M}$ \\
\hline RS1 & $\mathrm{RS} 2$ & 15 & 2.53 \\
\hline RS2 & RS3 & 50 & 2.4 \\
\hline \multicolumn{4}{|c|}{ ! Cysteine } \\
\hline SC & NCT & 20 & 2.65 \\
\hline $\mathrm{NC}$ & SC & 55 & 2.58 \\
\hline \multicolumn{4}{|c|}{ ! Glutamic Acid } \\
\hline Co01 & $\mathrm{C} 2 \mathrm{M}$ & 90.98 & 2.5 \\
\hline $\mathrm{C} 2 \mathrm{M}$ & NCT & 15 & 2.45 ! Same as Gln, Met \\
\hline $\mathrm{NC}$ & $\mathrm{C} 2 \mathrm{M}$ & 34 & 2.30 ! Same as Gln, Met \\
\hline
\end{tabular}


! Glutamine

$\begin{array}{llll}\text { CON1 } & \text { C2M } & 42 & 2.44 \\ \text { C2M } & \text { NCT } & 15 & 2.45 \text { ! Same as Glu, Met } \\ \text { NC } & \text { C2M } & 34 & 2.30 \text { ! Same as Glu, Met }\end{array}$

! Glycine

$\begin{array}{llll}\text { GNT } & \text { CO } & 50 & 2.50 \\ \text { CO } & \text { GNC } & 70 & 2.15 \\ \text { GNC } & \text { CO01 } & 60 & 2.50\end{array}$

! Histidine

NCR2 NCR1

$50 \quad 1.86$

NCR1 CCR1

$30 \quad 2.45$

NCR2 CCR1

$50 \quad 2.53$

CCR1 NCT

$30 \quad 2.35$

NC CCR1

$80 \quad 2.26$

! Isoleucine

C2E C2E2

$44 \quad 2.41$

C2E2 NCT

$40 \quad 2.50$

NC C2E2

$30 \quad 2.28$

! Leucine

$\begin{array}{llll}\text { C41 } & \text { NCT } & 12 & 3.18 \\ \text { NC } & \text { C41 } & 10 & 2.95\end{array}$

! Lysine

$\begin{array}{llll}\text { NC2 } & \text { C3M } & 20 & 3.07 \\ \text { C3M } & \text { NCT } & 5 & 2.90 \\ \text { NC } & \text { C3M } & 10 & 2.52\end{array}$

! Methionine

CS C2M

$32 \quad 2.5$

C2M NCT

$15 \quad 2.45$ ! Same as Glu, Gln

$\mathrm{NC} \quad \mathrm{C} 2 \mathrm{M}$

$34 \quad 2.30$ ! Same as Glu, Gln

! Phenylalanine

$\begin{array}{llll}\text { BZF1 } & \text { BZF2 } & 62 & 2.23 \\ \text { BZF1 } & \text { TL2F } & 55 & 2.875 \\ \text { BZF2 } & \text { TL2F } & 53 & 2.45 \\ \text { TL2F } & \text { NCT } & 7 & 2.75 \\ \text { NC } & \text { TL2F } & 17 & 2.43\end{array}$


! Proline

\begin{tabular}{|c|c|c|c|}
\hline CO & PNC & 40 & 2.04 \\
\hline PNC & PS & 45 & 1.77 \\
\hline \multicolumn{4}{|c|}{ ! Serine } \\
\hline COH1 & NCT & 30 & 2.5 \\
\hline $\mathrm{NC}$ & $\mathrm{COH} 1$ & 35 & 2.28 \\
\hline \multicolumn{4}{|c|}{ ! Threonine } \\
\hline $\mathrm{CCOH}$ & NCT & 55 & 2.58 \\
\hline NC & $\mathrm{CCOH}$ & 45 & 2.25 \\
\hline \multicolumn{4}{|c|}{ ! Tryptophan } \\
\hline TOL4 & PL1 & 60 & 3.11 \\
\hline TOL4 & TL2W & 40 & 3.358 \\
\hline TL2W & PL1 & 60 & 2.195 \\
\hline TL2W & NCT & 20 & 2.90 \\
\hline $\mathrm{NC}$ & TL2W & 10 & 2.50 \\
\hline
\end{tabular}

! Tyrosine

$\begin{array}{llll}\text { TL2Y } & \text { BZY } & 50 & 2.45 \\ \text { BZY } & \text { COH3 } & 50 & 2.45 \\ \text { COH3 } & \text { TL2Y } & 50 & 3.52 \\ \text { TL2Y } & \text { NCT } & 15 & 2.83 \\ \text { NC } & \text { TL2Y } & 15 & 2.42\end{array}$

! Valine

$\begin{array}{llll}\text { C3E2 } & \text { NCT } & 40 & 2.44 \\ \text { NC } & \text { C3E2 } & 35 & 2.26\end{array}$

! Backbone

$\begin{array}{lll}\text { NCT } & \text { CO } & 35\end{array}$

$\begin{array}{llll}\mathrm{CO} & \mathrm{NC} & 60 & 2.08\end{array}$

$\begin{array}{llll}\mathrm{NC} & \mathrm{COO} 1 & 70 & 2.48\end{array}$

! Carbon-Carbon \& Carbon-Peptide

$\begin{array}{llll}\text { C3E } & \text { C3M } & 37.695 & 3.561 \\ \text { C3M } & \text { C3M } & 37.695 & 3.561 \text { ! from C3E-C3M } \\ \text { C3M } & \text { CO } & 38.537 & 2.914 \text { ! from C2M-C3M } \\ \text { C2E } & \text { C2M } & 35.293 & 2.561 \\ \text { C2M } & \text { C2M } & 36.609 & 2.520\end{array}$




\begin{tabular}{|c|c|c|c|c|c|}
\hline \multicolumn{6}{|c|}{ ANGLES } \\
\hline \multicolumn{6}{|c|}{ ! } \\
\hline \multicolumn{6}{|c|}{ !V(angle $)=$ Ktheta $(\text { Theta }- \text { Theta } 0)^{* * 2}$} \\
\hline \multicolumn{6}{|c|}{ ! } \\
\hline \multicolumn{6}{|c|}{ !Ktheta: kcal/mole/rad**2 } \\
\hline \multicolumn{6}{|c|}{ !Theta0: degrees } \\
\hline \multicolumn{6}{|c|}{ ! } \\
\hline \multicolumn{3}{|c|}{ !bead types } & \multicolumn{3}{|c|}{ Ktheta Theta0 } \\
\hline \multicolumn{6}{|c|}{ ! Alanine } \\
\hline NCC1 & $\mathrm{CO}$ & NCC2 & 20 & 97 & \\
\hline $\mathrm{CO}$ & NCC2 & $\mathrm{CO}$ & 5 & 91 & \\
\hline NCC2 & $\mathrm{CO}$ & NCC2 & 17 & 96 & \\
\hline $\mathrm{CO}$ & NCC2 & C001 & 6 & 83 & \\
\hline \multicolumn{6}{|c|}{ ! Asparagine } \\
\hline CON2 & NCT & $\mathrm{CO}$ & 6 & 68 & \\
\hline $\mathrm{CO}$ & NC & CON2 & 3 & 138 & \\
\hline CON2 & NC & $\mathrm{CO01}$ & 50 & 114 & \\
\hline \multicolumn{6}{|c|}{ ! Aspartic Acid } \\
\hline COO2 & NCT & $\mathrm{CO}$ & 5 & 75 & \\
\hline $\mathrm{CO}$ & $\mathrm{NC}$ & $\mathrm{COO} 2$ & 2 & 138 & \\
\hline COO2 & $\mathrm{NC}$ & C001 & 27 & 114 & \\
\hline \multicolumn{6}{|c|}{ ! Arginine } \\
\hline $\mathrm{CO}$ & $\mathrm{NC}$ & RS1 & 10 & 140 & \\
\hline RS1 & NC & Co01 & 20 & 83 & \\
\hline $\mathrm{NC}$ & RS1 & $\mathrm{RS} 2$ & 10 & 153 & \\
\hline RS1 & $\mathrm{RS} 2$ & RS3 & 5 & 157 & \\
\hline \multicolumn{6}{|c|}{ ! Cysteine } \\
\hline SC & NCT & $\mathrm{CO}$ & 10 & 71 & \\
\hline $\mathrm{CO}$ & NC & $\mathrm{SC}$ & 30 & 166 & \\
\hline SC & NC & Coo1 & 35 & 84 & \\
\hline \multicolumn{6}{|c|}{ ! Glutamic Acid } \\
\hline C001 & $\mathrm{C} 2 \mathrm{M}$ & NCT & 25 & 114 & \\
\hline $\mathrm{C} 2 \mathrm{M}$ & NCT & $\mathrm{CO}$ & 10 & 73 & ! Same in Gln, Met \\
\hline $\mathrm{CO}$ & NC & $\mathrm{C} 2 \mathrm{M}$ & 12 & 173 & ! Same in Gln, Met \\
\hline NC & $\mathrm{C} 2 \mathrm{M}$ & C001 & 25 & 162 & \\
\hline $\mathrm{C} 2 \mathrm{M}$ & NC & Co01 & 20 & 83 & ! Same in Gln, Met \\
\hline
\end{tabular}


! Glutamine

$\begin{array}{llllll}\text { CON1 } & \text { C2M } & \text { NCT } & 30 & 100 & \\ \text { C2M } & \text { NCT } & \text { CO } & 10 & 73 & \text { ! Same in Gln, Met } \\ \text { CO } & \text { NC } & \text { C2M } & 12 & 173 & \text { ! Same in Gln, Met } \\ \text { NC } & \text { C2M } & \text { CON1 } & 30 & 160 & \\ \text { C2M } & \text { NC } & \text { COO1 } & 20 & 83 & \text { ! Same in Gln, Met }\end{array}$

! Glycine

GNT CO GNC $\quad 30 \quad 90$

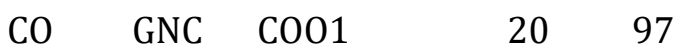

! Histidine

$\begin{array}{llllll}\text { NCR2 } & \text { CCR1 } & \text { NCR1 } & 40 & 44 & \text { ! This and next } 3 \text { must add to 180deg } \\ \text { CCR1 } & \text { NCR1 } & \text { NCR2 } & 25 & 70 \\ \text { NCR1 } & \text { NCR2 } & \text { CCR1 } & 25 & 66 \\ \text { NCR1 } & \text { CCR1 } & \text { NCT } & 5 & 140 \\ \text { CCR1 } & \text { NCT } & \text { CO } & 40 & 68 \\ \text { CO } & \text { NC } & \text { CCR1 } & 1 & 140 \\ \text { CO01 } & \text { NC } & \text { CCR1 } & 100 & 90 \\ \text { NC } & \text { CCR1 } & \text { NCR2 } & 7 & 124 \\ \text { NCR2 } & \text { CCR1 } & \text { NCT } & 10 & 135 \\ \text { NC } & \text { CCR1 } & \text { NCR1 } & 7 & 120\end{array}$

! Isoleucine

$\begin{array}{lllll}\text { C2E } & \text { C2E2 } & \text { NCT } & 10 & 70 \\ \text { C2E2 } & \text { NCT } & \text { CO } & 30 & 70 \\ \text { CO } & \text { NC } & \text { C2E2 } & 10 & 146 \\ \text { NC } & \text { C2E2 } & \text { C2E } & 20 & 70 \\ \text { C2E2 } & \text { NC } & \text { COO1 } & 40 & 100\end{array}$

! Leucine

$\begin{array}{lllll}\text { C41 } & \text { NCT } & \text { CO } & 6 & 73 \\ \text { CO } & \text { NC } & \text { C41 } & 2 & 144 \\ \text { C41 } & \text { NC } & \text { C001 } & 6 & 110\end{array}$

! Lysine

$\begin{array}{lllll}\text { NC2 } & \text { C3M } & \text { NCT } & 3 & 140 \\ \text { C3M } & \text { NCT } & \text { CO } & 3 & 75 \\ \text { CO } & \text { NC } & \text { C3M } & 1 & 140 \\ \text { NC } & \text { C3M } & \text { NC2 } & 5 & 140 \\ \text { C3M } & \text { NC } & \text { COO1 } & 40 & 106\end{array}$




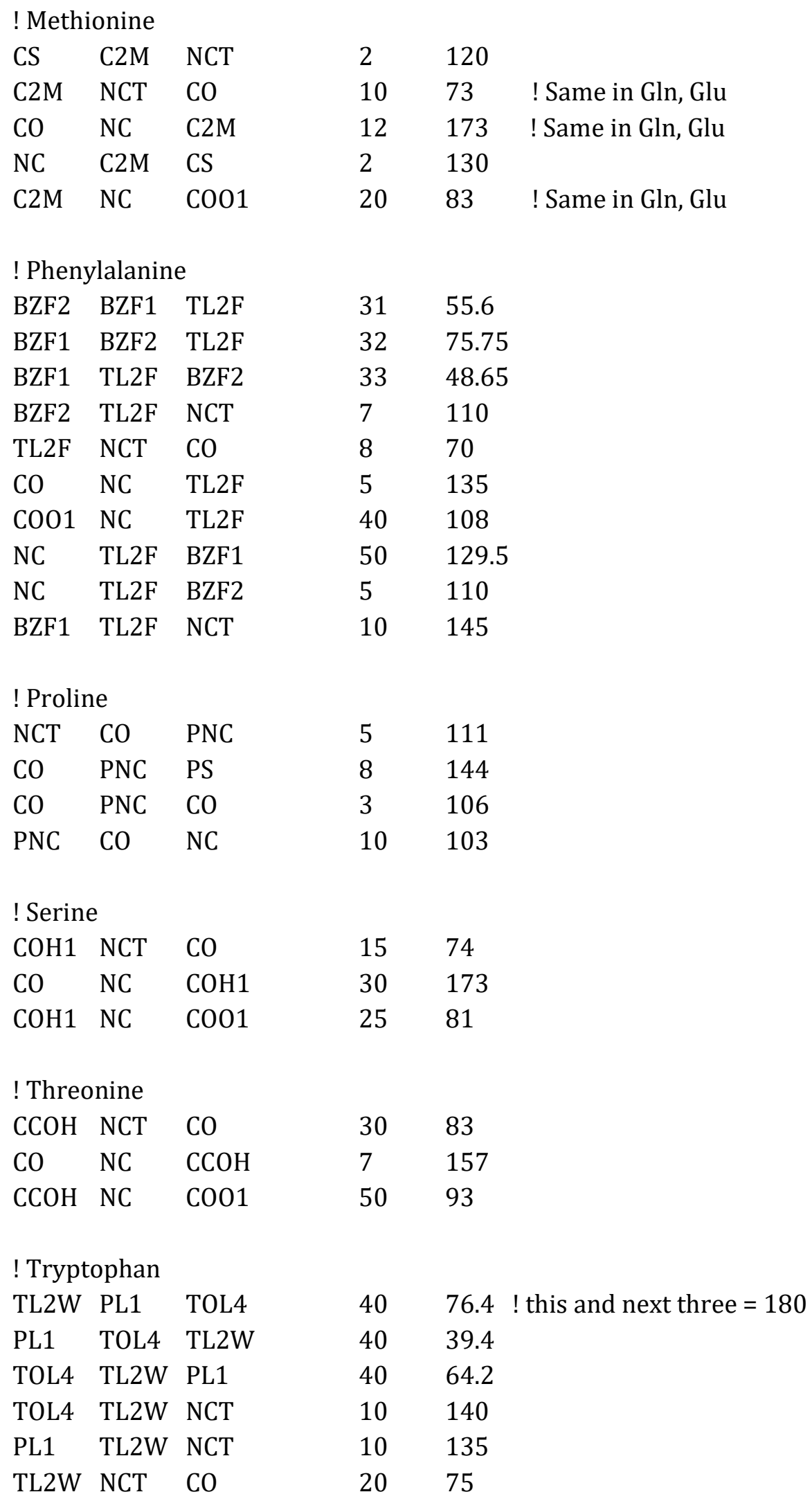




\begin{tabular}{|c|c|c|c|c|c|}
\hline \multicolumn{6}{|c|}{ ! Tyrosine } \\
\hline TL2Y & $\mathrm{BZY}$ & $\mathrm{COH} 3$ & 25 & \multicolumn{2}{|l|}{92} \\
\hline BZY & СOH3 & TL2Y & 25 & \multicolumn{2}{|l|}{44} \\
\hline $\mathrm{COH} 3$ & TL2Y & BZY & 25 & \multicolumn{2}{|l|}{44} \\
\hline BZY & TL2Y & NCT & 5 & \multicolumn{2}{|l|}{117} \\
\hline $\mathrm{COH} 3$ & TL2Y & NCT & 15 & \multicolumn{2}{|l|}{145} \\
\hline TL2Y & NCT & $\mathrm{CO}$ & 15 & \multicolumn{2}{|l|}{67} \\
\hline TL2Y & NC & C001 & 12 & \multicolumn{2}{|l|}{108} \\
\hline $\mathrm{CO}$ & NC & TL2Y & 2 & \multicolumn{2}{|l|}{123} \\
\hline $\mathrm{BZY}$ & TL2Y & $\mathrm{NC}$ & 2 & \multicolumn{2}{|l|}{110} \\
\hline $\mathrm{COH} 3$ & TL2Y & NC & 20 & \multicolumn{2}{|l|}{128} \\
\hline \multicolumn{6}{|c|}{ ! Valine } \\
\hline C3E2 & NCT & $\mathrm{CO}$ & 20 & \multicolumn{2}{|l|}{75} \\
\hline $\mathrm{CO}$ & $\mathrm{NC}$ & C3E2 & 2 & \multicolumn{2}{|l|}{160} \\
\hline C3E2 & $\mathrm{NC}$ & C001 & 28 & \multicolumn{2}{|l|}{77} \\
\hline \multicolumn{6}{|c|}{ ! Carbon-Carbon \& Carbon-Peptide } \\
\hline C3E & C3M & C3M & 3.4 & 145 & ! based on C3E-C3M-C3E \\
\hline C3M & $\mathrm{C} 3 \mathrm{M}$ & C3M & 3.4 & 145 & ! based on C3E-C3M-C3E \\
\hline C3M & $\mathrm{C} 3 \mathrm{M}$ & $\mathrm{CO}$ & 3.4 & 145 & ! based on C3E-C3M-C3E \\
\hline C3M & $\mathrm{CO}$ & NCC2 & 1 & 140 & ! from Lys CO-NC-C3M \\
\hline $\mathrm{C} 2 \mathrm{E}$ & $\mathrm{C} 2 \mathrm{M}$ & $\mathrm{C} 2 \mathrm{M}$ & 3.886 & \multicolumn{2}{|c|}{147.766} \\
\hline $\mathrm{C} 2 \mathrm{M}$ & $\mathrm{C} 2 \mathrm{M}$ & $\mathrm{C} 2 \mathrm{M}$ & 3.460 & \multicolumn{2}{|c|}{144.226} \\
\hline \multicolumn{6}{|c|}{ ! Backbone } \\
\hline NCT & $\mathrm{CO}$ & NC & 7 & \multicolumn{2}{|l|}{90} \\
\hline $\mathrm{CO}$ & NC & C001 & 1 & \multicolumn{2}{|l|}{100} \\
\hline NC & $\mathrm{CO}$ & $\mathrm{NC}$ & 8 & 103 & ! from valylvalylvaline \\
\hline $\mathrm{CO}$ & NC & $\mathrm{CO}$ & 1 & 120 & ! from valylvalylvaline \\
\hline NC & $\mathrm{CO}$ & NCC2 & 20 & 97 & ! from NCC1 CO NCC2 (alanine) \\
\hline \multicolumn{6}{|c|}{ DIHEDRALS } \\
\hline \multicolumn{6}{|c|}{$!$} \\
\hline \multicolumn{6}{|c|}{$! \mathrm{V}($ dihedral $)=\operatorname{Kchi}(1+\cos (\mathrm{n}(\mathrm{chi})-$ delta $))$} \\
\hline \multicolumn{6}{|l|}{ ! } \\
\hline \multicolumn{6}{|c|}{ !Kchi: kcal/mole } \\
\hline \multicolumn{6}{|c|}{ !n: multiplicity } \\
\hline \multicolumn{6}{|c|}{ !delta: degrees } \\
\hline
\end{tabular}


! bead types

! Alanine

NCC1 CO NCC2 CO

CO NCC2 CO NCC2

NCC2 CO NCC2 COO1

! Asparagine

NS NCT CO NC

NCT CO NC NS

! Aspartic Acid

NCT CO NC DS

! Arginine

ANT CO NC RS1

CO NC RS1 RS2

C001 NC RS1 RS2

NC RS1 RS2 RS3

! Cysteine

CS NCT CO NC

NCT CO NC CS

! Glutamic Acid

CO01 C2M NCT CO

C2M NCT CO NC

NCT $\mathrm{CO} \quad \mathrm{NC} \quad \mathrm{C} 2 \mathrm{M}$

$\begin{array}{llll}\mathrm{CO} & \mathrm{NC} & \mathrm{C} 2 \mathrm{M} & \mathrm{COO} 1\end{array}$

$\begin{array}{llll}\mathrm{C} 001 & \mathrm{C} 2 \mathrm{M} & \mathrm{NC} & \mathrm{CO} 1\end{array}$

! Glutamine

CON1 C2M NCT CO

C2M NCT CO NC

NCT $\mathrm{CO}$ NC $\mathrm{C} 2 \mathrm{M}$

$\begin{array}{llll}\mathrm{CO} & \mathrm{NC} & \mathrm{C} 2 \mathrm{M} & \mathrm{CON} 1\end{array}$

CON1 C2M NC CO01

! Glycine

GNT CO GNC COO1
Kchi n delta

$\begin{array}{lll}1.0 & 1 & 90\end{array}$

$\begin{array}{lll}0.6 & 1 & 0\end{array}$

$\begin{array}{lll}1.2 & 1 & 0\end{array}$

$\begin{array}{lll}0.3 & 2 & 0\end{array}$

$\begin{array}{lll}1.45 & 1 & 100\end{array}$

$\begin{array}{lll}0.3 & 2 & 0\end{array}$

$\begin{array}{lll}1.7 & 1 & 102\end{array}$

$\begin{array}{lll}0.7 & 2 & 180\end{array}$

$\begin{array}{lll}0.7 & 2 & 0\end{array}$

$\begin{array}{lll}0.7 & 1 & 180\end{array}$

$\begin{array}{lll}0.7 & 2 & 0\end{array}$

$\begin{array}{lll}1.0 & 1 & 0\end{array}$

$\begin{array}{lll}0.6 & 2 & 0\end{array}$

$\begin{array}{lll}1.0 & 2 & 0\end{array}$

$\begin{array}{lll}0.7 & 2 & 0\end{array}$

$\begin{array}{lll}0.7 & 2 & 0\end{array}$

$\begin{array}{lll}1.0 & 1 & 90\end{array}$

$\begin{array}{lll}1.0 & 1 & 90\end{array}$

$\begin{array}{lll}0.5 & 2 & 0\end{array}$

$\begin{array}{lll}0.7 & 2 & 0\end{array}$

$\begin{array}{lll}0.7 & 2 & 0\end{array}$

$0.522 \quad 0$

$\begin{array}{lll}0.5 & 1 & 180\end{array}$

$\begin{array}{lll}0.5 & 2 & 0\end{array}$ 


\begin{tabular}{lllllll}
\multicolumn{2}{l}{ Histidine } \\
NCR1 & CCR1 & NCT & CO & 0.1 & 3 & 180 \\
NCR2 & CCR1 & NCT & CO & 0.1 & 1 & 180 \\
CCR1 & NCT & CO & NC & 0.1 & 2 & 81 \\
NCT & CO & NC & CCR1 & 0.5 & 1 & 60 \\
CO & NC & CCR1 & NCR2 & 0.5 & 1 & 180 \\
CO & NC & CCR1 & NCR1 & 0.5 & 1 & 180 \\
CO01 & NC & CCR1 & NCR2 & 0.8 & 1 & 0 \\
C001 & NC & CCR1 & NCR1 & 0.5 & 1 & 0
\end{tabular}

! Isoleucine

$\begin{array}{lllllll}\text { C2E } & \text { C2E2 } & \text { NCT } & \text { CO } & 0.5 & 1 & 0 \\ \text { C2E2 } & \text { NCT } & \text { CO } & \text { NC } & 0.5 & 2 & 0 \\ \text { NCT } & \text { CO } & \text { NC } & \text { C2E2 } & 0.5 & 1 & 90 \\ \text { CO } & \text { NC } & \text { C2E2 } & \text { C2E } & 0.5 & 1 & 270 \\ \text { C2E } & \text { C2E2 } & \text { NC } & \text { CO01 } & 0.5 & 2 & 180\end{array}$

! Leucine

$\begin{array}{lllllll}\text { C41 } & \text { NCT } & \text { CO } & \text { NC } & 0.5 & 1 & 0 \\ \text { NCT } & \text { CO } & \text { NC } & \text { C41 } & 0.6 & 1 & 90\end{array}$

! Lysine

$\begin{array}{lllllll}\text { NC2 } & \text { C3M } & \text { NCT } & \text { CO } & 0.2 & 2 & 76 \\ \text { C3M } & \text { NCT } & \text { CO } & \text { NC } & 0.7 & 1 & 90 \\ \text { NCT } & \text { CO } & \text { NC } & \text { C3M } & 2.0 & 1 & 120 \\ \text { CO } & \text { NC } & \text { C3M } & \text { NC2 } & 0.2 & 3 & 180 \\ \text { NC2 } & \text { C3M } & \text { NC } & \text { CO01 } & 0.1 & 3 & 0\end{array}$

! Methionine

$\begin{array}{lllllll}\text { CS } & \text { C2M } & \text { NCT } & \text { CO } & 0.5 & 1 & 0 \\ \text { C2M } & \text { NCT } & \text { CO } & \text { NC } & 0.7 & 2 & 0 \\ \text { NCT } & \text { CO } & \text { NC } & \text { C2M } & 0.7 & 2 & 0 \\ \text { CO } & \text { NC } & \text { C2M } & \text { CS } & 0.5 & 1 & 0 \\ \text { CS } & \text { C2M } & \text { NC } & \text { COO1 } & 0.4 & 1 & 60\end{array}$

! Phenylalanine

$\begin{array}{lllllll}\text { BZF1 } & \text { TL2F } & \text { NCT } & \text { CO } & 0.3 & 2 & 0 \\ \text { BZF2 } & \text { TL2F } & \text { NCT } & \text { CO } & 0.4 & 2 & 0 \\ \text { TL2F } & \text { NCT } & \text { CO } & \text { NC } & 0.3 & 2 & 76 \\ \text { NCT } & \text { CO } & \text { NC } & \text { TL2F } & 1 & 1 & 90 \\ \text { CO } & \text { NC } & \text { TL2F } & \text { BZF1 } & 0.5 & 1 & 270 \\ \text { CO } & \text { NC } & \text { TL2F } & \text { BZF2 } & 0.3 & 1 & 120 \\ \text { BZF1 } & \text { TL2F } & \text { NC } & \text { CO01 } & 0.3 & 1 & 270\end{array}$


$\begin{array}{lllllll}\text { BZF2 } & \text { TL2F } & \text { NC } & \text { COO1 } & 0.5 & 2 & 180\end{array}$

! Proline

$\begin{array}{lllllll}\text { C3E2 } & \text { NCT } & \text { CO } & \text { PNC } & 1.0 & 2 & 0 \\ \text { NCT } & \text { CO } & \text { PNC } & \text { PS } & 0.3 & 1 & 60 \\ \text { NCT } & \text { CO } & \text { PNC } & \text { CO } & 0.3 & 1 & 60 \\ \text { CO } & \text { PNC } & \text { CO } & \text { NC } & 0.5 & 1 & 180 \\ \text { PS } & \text { PNC } & \text { CO } & \text { NC } & 0.3 & 2 & 0 \\ \text { PNC } & \text { CO } & \text { NC } & \text { C41 } & 0.5 & 1 & 60 \\ \text { PNC } & \text { CO } & \text { NC } & \text { COO1 } & 1.7 & 1 & 0\end{array}$

! Serine

$\begin{array}{lllllll}\mathrm{COH} 1 & \mathrm{NCT} & \mathrm{CO} & \mathrm{NC} & 0.5 & 2 & 0\end{array}$

$\begin{array}{lllllll}\text { NCT } & \text { CO } & \text { NC } & \text { COH1 } & 0.8 & 1 & 0\end{array}$

! Threonine

$\begin{array}{lllllll}\mathrm{CCOH} & \mathrm{NCT} & \mathrm{CO} & \mathrm{NC} & 0.3 & 2 & 0\end{array}$

$\begin{array}{lllllll}\text { NCT } & \mathrm{CO} & \mathrm{NC} & \mathrm{CCOH} & 1 & 1 & 90\end{array}$

! Tryptophan

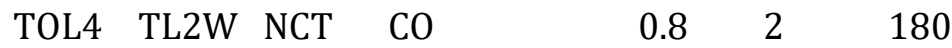

$\begin{array}{llllll}\text { PL1 TL2W } & \text { NCT } & \text { CO } & 0.7 & 2 & 0\end{array}$

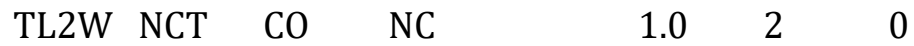

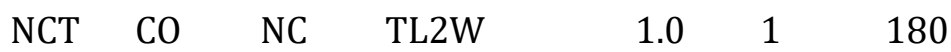

$\begin{array}{llllll}\text { CO } & \text { NC } & \text { TL2W PL1 } & 1.0 & 1 & 180\end{array}$

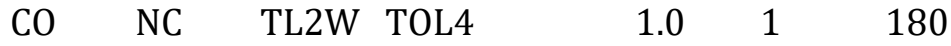

$\begin{array}{llllll}\text { CO01 NC } & \text { TL2W PL1 } & 1.2 & 1 & 0\end{array}$

$\begin{array}{llllll}\text { CO01 NC } & \text { TL2W } & \text { TOL4 } & 1.3 & 1 & 0\end{array}$

! Tyrosine

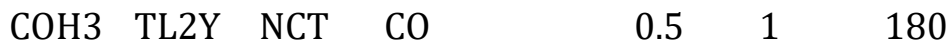

$\begin{array}{lllllll}\text { BZY } & \text { TL2Y } & \text { NCT } & \text { CO } & 0.5 & 1 & 180\end{array}$

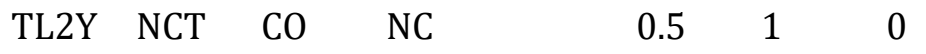

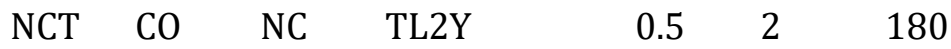

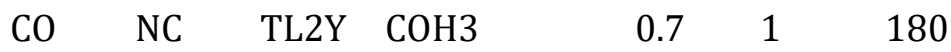

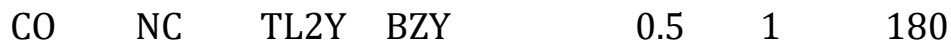

$\begin{array}{lllllll}\text { COH3 } & \text { TL2Y } & \text { NC } & \text { COO1 } & 1.0 & 1 & 0\end{array}$

$\begin{array}{lllllll}\text { BZY } & \text { TL2Y } & \text { NC } & \text { COO1 } & 0.5 & 1 & 0\end{array}$

! Valine

$\begin{array}{lllllll}\text { C3E2 } & \text { NCT } & \text { CO } & \text { NC } & 1.3 & 2 & 90\end{array}$

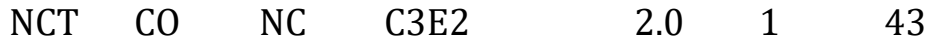


! Backbone

$\begin{array}{llllllll}\text { NCT } & \text { CO } & \text { NC } & \text { COO1 } & 0.5 & 1 & 0 & \\ \text { NCT } & \text { CO } & \text { NC } & \text { CO } & 2.0 & 1 & 0 & \text { ! from valylvalylvaline (t8) } \\ \text { CO } & \text { NC } & \text { CO } & \text { NC } & 0.1 & 2 & 0 & \text { ! from valylvalylvaline (t8) } \\ \text { NC } & \text { CO } & \text { NC } & \text { COO1 } & 2.5 & 1 & 0 & \text { ! from valylvalylvaline (t8) } \\ & & & & & & & \\ \text { X } & \text { X } & \text { X } & \text { X } & 0 & 0 & 0 & \text { ! sets all others not listed to 0 }\end{array}$

! So all dihedrals like LS1-NC-CO-NC and C3M-C3M-C3M are set to 0!

\begin{tabular}{|c|c|c|c|c|}
\hline \multicolumn{5}{|l|}{ NON } \\
\hline \multicolumn{5}{|l|}{$!$} \\
\hline \multirow{2}{*}{\multicolumn{5}{|c|}{ 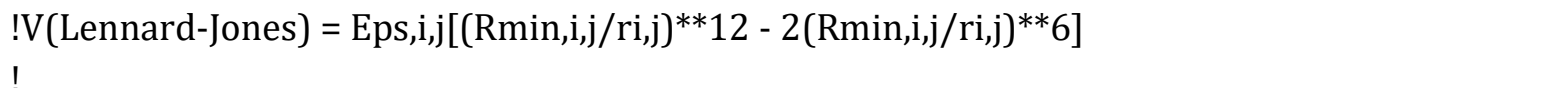 }} \\
\hline & & & & \\
\hline \multicolumn{5}{|c|}{ !epsilon: kcal/mole, Eps,i,j = sqrt(eps,i * eps,j) } \\
\hline \multicolumn{5}{|c|}{$! R \min / 2: \mathrm{A}, \mathrm{Rmin}, \mathrm{i}, \mathrm{j}=\mathrm{Rmin} / 2, \mathrm{i}+\mathrm{Rmin} / 2, \mathrm{j}$} \\
\hline \multicolumn{5}{|c|}{ ! } \\
\hline !bead & ignored & epsilon & $\operatorname{Rmin} / 2$ & \\
\hline CCR1 & 0.000000 & -0.3058 & 2.4725 & \\
\hline NCR1 & 0.000000 & -0.7506 & 1.8759 & \\
\hline NCR2 & 0.000000 & -0.4417 & 2.1546 & \\
\hline NCT & 0.000000 & -0.6000 & 2.1650 & ! same as NC2 (Lys bead) \\
\hline $\mathrm{CO}$ & 0.000000 & -0.6690 & 2.4361 & \\
\hline NC & 0.000000 & -0.6765 & 2.1436 & \\
\hline Co01 & 0.000000 & -1.2516 & 2.2088 & ! from Yaxin's propionic acid bead \\
\hline NCC1 & 0.000000 & -0.8699 & 2.4014 & \\
\hline NCC2 & 0.000000 & -0.8699 & 2.4014 & \\
\hline C41 & 0.000000 & -0.7120 & 2.8515 & \\
\hline NC2 & 0.000000 & -0.6000 & 2.1650 & \\
\hline CON2 & 0.000000 & -1.1332 & 2.4343 & \\
\hline COO2 & 0.000000 & -0.8714 & 2.3950 & \\
\hline RS1 & 0.000000 & -0.3420 & 2.4343 & ! Arg side chain; same as C2M \\
\hline RS2 & 0.000000 & -0.6765 & 2.1436 & ! Arg side chain; same as NC bead \\
\hline RS3 & 0.000000 & -1.0881 & 2.2635 & ! Arg side chain \\
\hline SC & 0.000000 & -0.8638 & 2.3606 & \\
\hline CON1 & 0.000000 & -1.0876 & 2.0609 & \\
\hline GNT & 0.000000 & -0.6000 & 2.1650 & ! same as NCT \\
\hline GNC & 0.000000 & -0.6765 & 2.1436 & ! same as NC \\
\hline C2E2 & 0.000000 & -0.3544 & 2.4146 & \\
\hline $\mathrm{CS}$ & 0.000000 & -0.7001 & 2.3969 & \\
\hline BZF1 & 0.000000 & -0.3205 & 2.2332 & ! from Karteek's benzene model \\
\hline BZF2 & 0.000000 & -0.3205 & 2.2332 & ! from Karteek's benzene model \\
\hline
\end{tabular}




$\begin{array}{lllll}\text { TOL2 } & 0.000000 & -0.5003 & 2.5318 & \\ \text { TL2F } & 0.000000 & -0.5003 & 2.5318 & \\ \text { TL2W } & 0.000000 & -0.5003 & 2.5318 & \\ \text { TL2Y } & 0.000000 & -0.5003 & 2.5318 & \\ \text { PNC } & 0.000000 & -0.6000 & 2.1650 & \text { ! Same as NC2 } \\ \text { PS } & 0.000000 & -0.5545 & 2.6010 & \text { ! Same as C3M } \\ \text { COH1 } & 0.000000 & -0.7152 & 2.0909 & \\ \text { CCOH } & 0.000000 & -0.7999 & 2.3741 & \\ \text { TOL4 } & 0.000000 & -0.5839 & 2.6920 & \\ \text { PL1 } & 0.000000 & -1.0688 & 1.8769 & \\ \text { COH3 } & 0.000000 & -0.7359 & 2.0730 & \\ \text { BZY } & 0.000000 & -0.3205 & 2.2332 & \text { ! from Karteek's benzene model } \\ \text { C3E2 } & 0.000000 & -0.4599 & 2.7807 & \\ \text { C2E } & 0.000000 & -0.3710 & 2.4343 & \text { ! Yaxin's parameters } \\ \text { C2M } & 0.000000 & -0.3420 & 2.4343 & \text { ! Yaxin's parameters } \\ \text { C3M } & 0.000000 & -0.5545 & 2.6010 & \text { ! Yaxin's parameters } \\ \text { C3E } & 0.000000 & -0.5927 & 2.6010 & \text { ! Yaxin's parameters } \\ \text { H2O1 } & 0.000000 & -1.1425 & 2.1170 & \end{array}$

\begin{tabular}{|c|c|c|c|}
\hline \multicolumn{4}{|c|}{ NBFIX } \\
\hline \multicolumn{2}{|c|}{ ! Bead } & Emin & Rmin \\
\hline \multicolumn{2}{|c|}{ ! Types } & (kcal $/ \mathrm{mol}$ ) & $(\AA ̊)$ \\
\hline C2E & H2O1 & -0.5130 & 4.2359 \\
\hline $\mathrm{C} 2 \mathrm{M}$ & H2O1 & -0.4400 & 4.2359 \\
\hline C3E & H2O1 & -0.6202 & 4.5000 \\
\hline C3M & H201 & -0.5434 & 4.5000 \\
\hline
\end{tabular}

END 


\section{D3: NAMD All-Atom Simulation Configuration File Example}

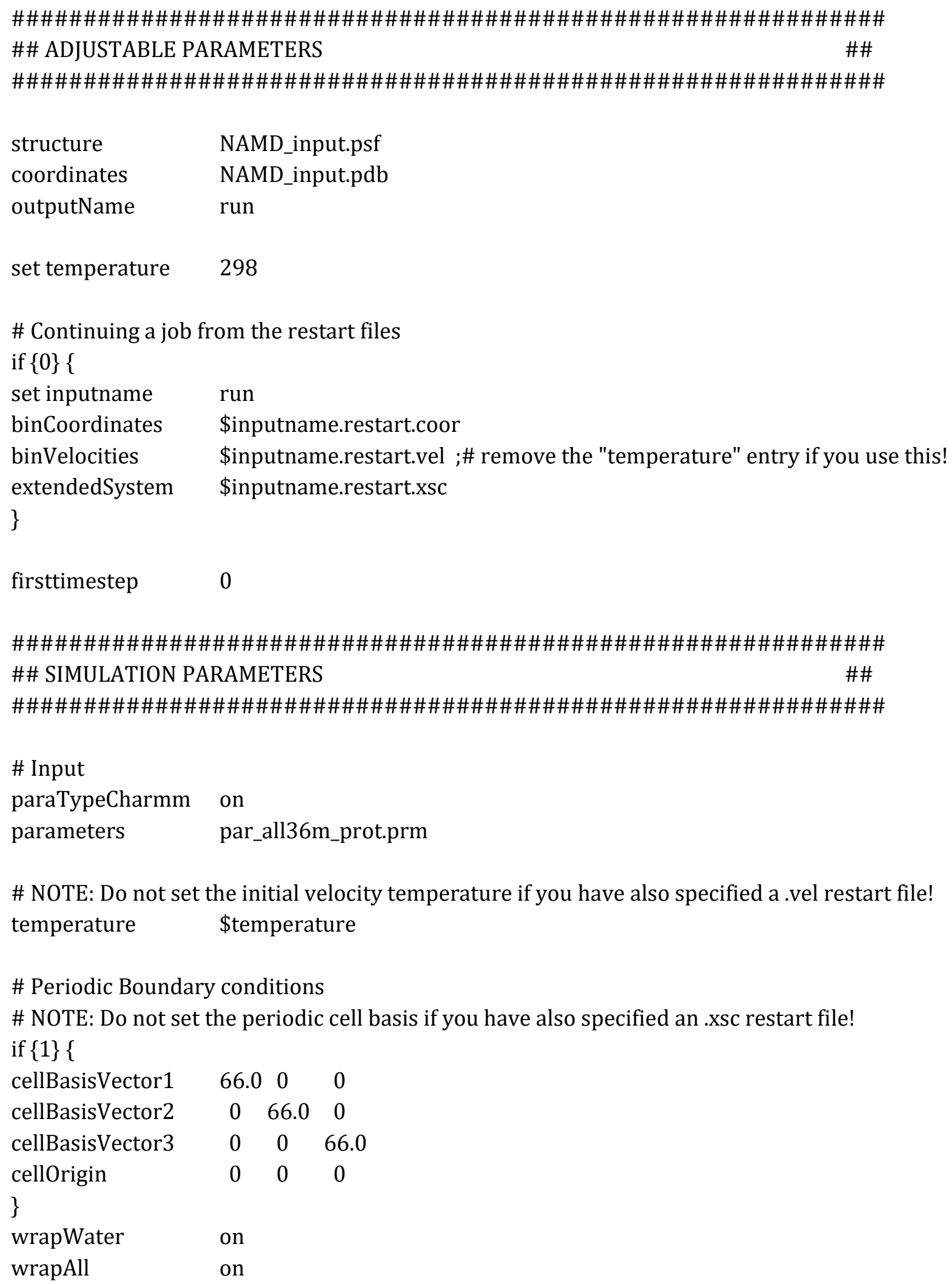




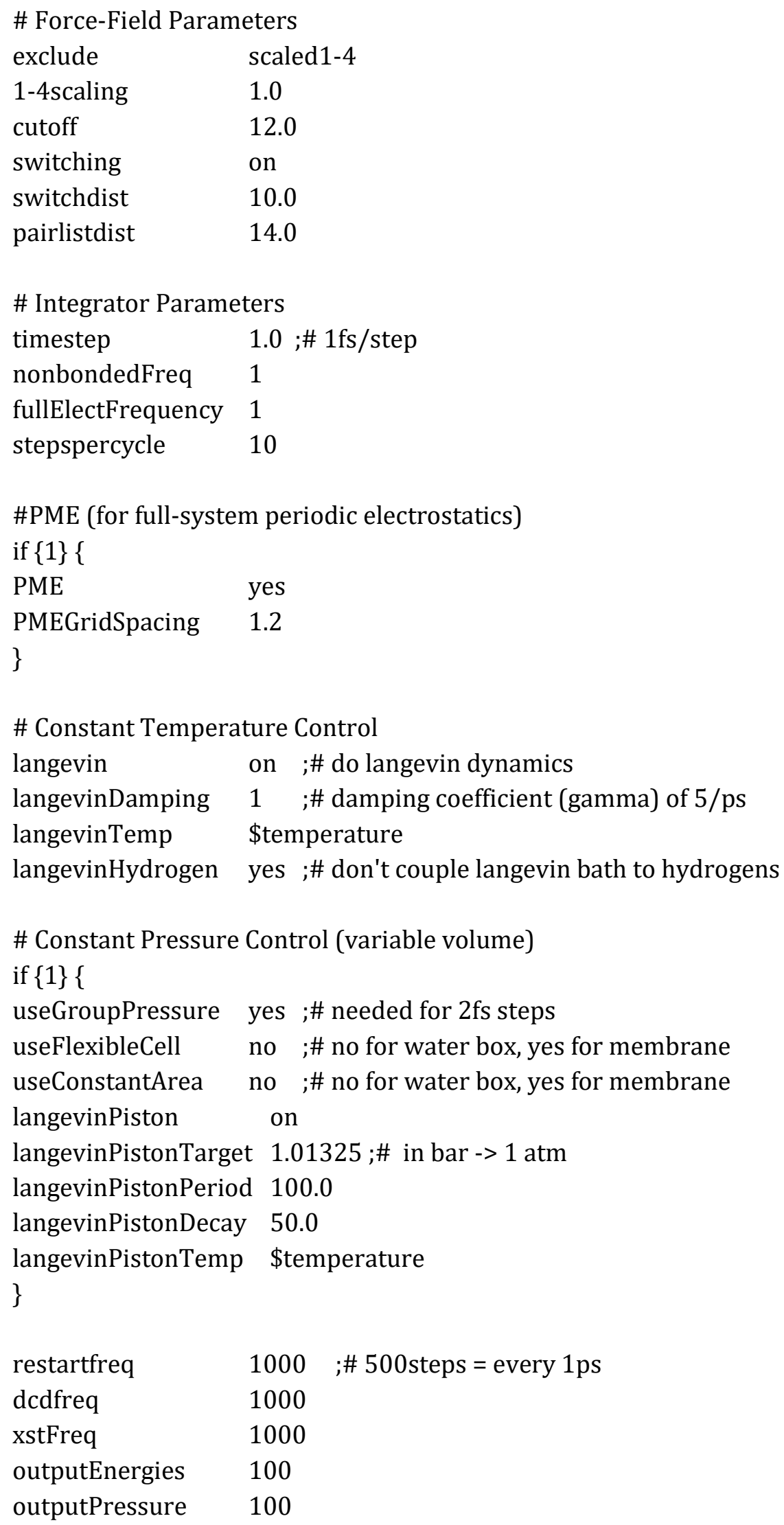




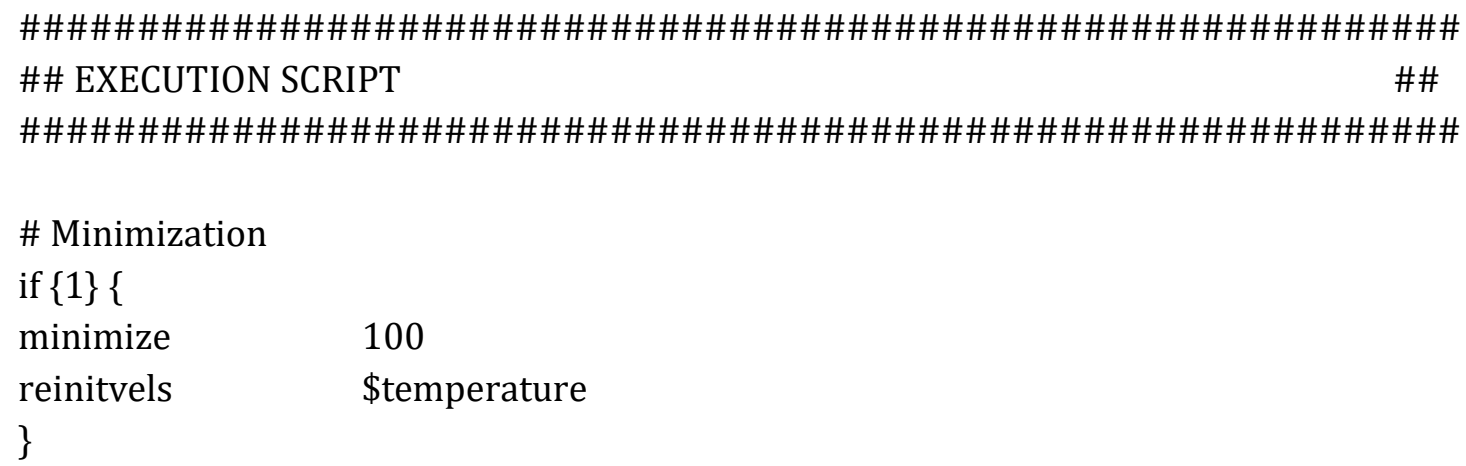

run $5000000 ; \# 1$ million femtoseconds = 1 ns 